\title{
The role of adipose tissue circadian clocks in metabolic maintenance
}

\author{
Dissertation \\ for the award of the degree \\ "Doctor rerum naturalium" \\ Division of Mathematics and Natural Sciences \\ of the Georg-August-Universität Göttingen
}

submitted by

Anton Shostak

from Minsk, Belarus 
Thesis Committee:

Prof. Dr. Henrik Oster, Medical Department I

University of Lübeck

Prof. Dr. Ahmed Mansouri, Molecular Cell Differentiation Group

Max Planck Institute for Biophysical Chemistry Göttingen

Prof. Dr. Ernst Wimmer, Department of Developmental Biology

The Göttingen Center for Molecular Biosciences (GZMB), Georg-August-Universität Göttingen

Date of oral examination: 12.11 .12 


\section{Declaration}

Herewith, I confirm that I have written the present PhD thesis independently and with no other sources and aids than quoted. 


\section{Content}

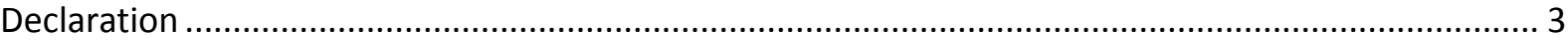

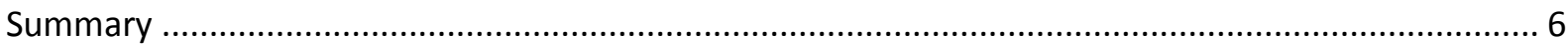

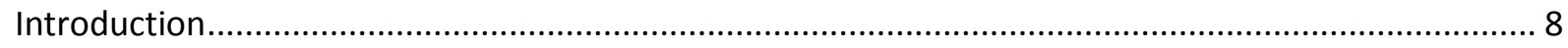

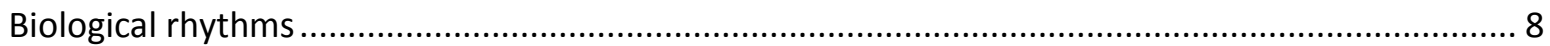

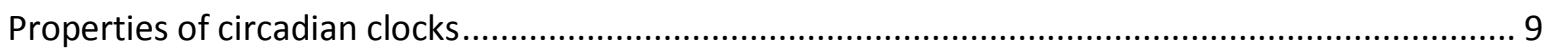

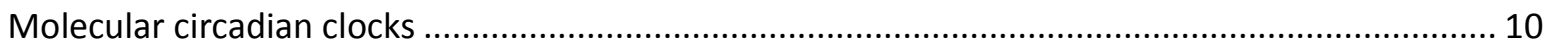

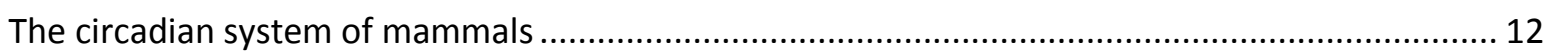

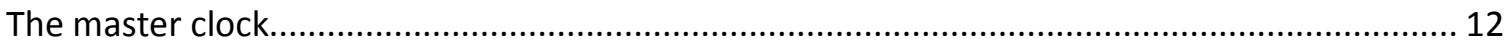

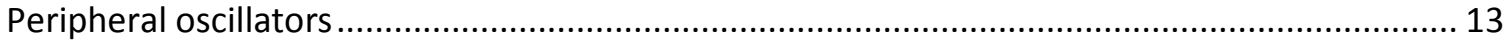

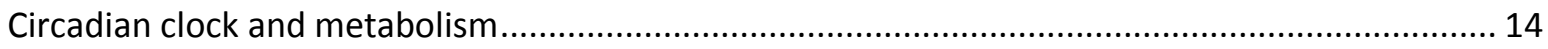

Metabolic phenotypes of circadian clock mutant mice ................................................................ 15

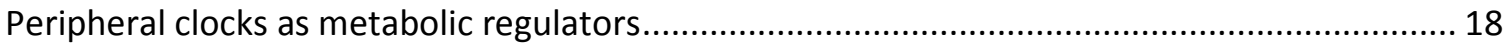

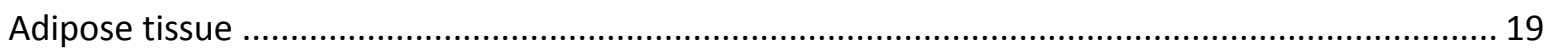

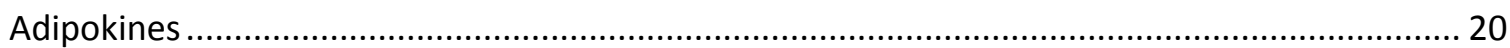

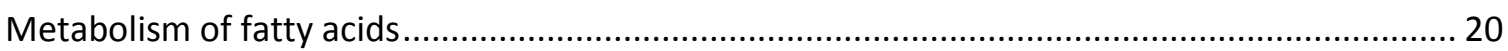

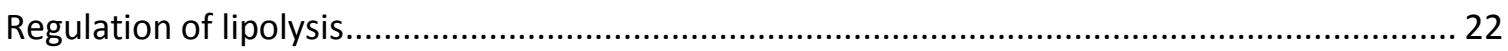

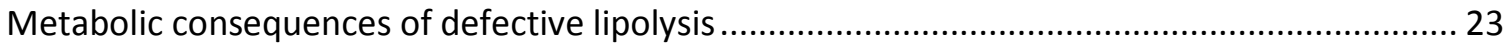

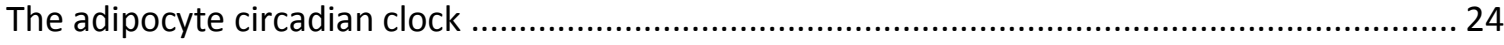

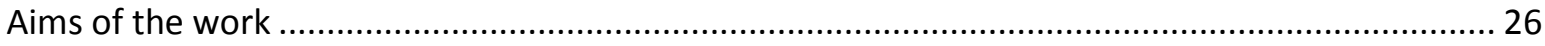

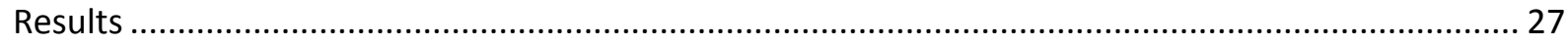

Circadian regulation of lipid mobilization in white adipose tissues............................................. 27

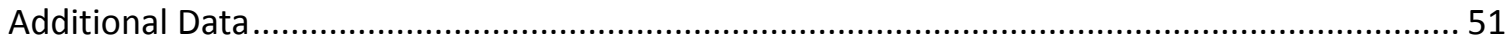

Generation of adipocyte-targeted circadian clock deficient mutant mice. ..................................... 52

Fabp4-cre Bmal1 fl/fl animals carry a deletion of Bmal1 in adipose tissue ................................. 52

Reduction of BMAL1 in the brain of Fabp4-cre Bmal1 fl/fl mice ............................................... 52

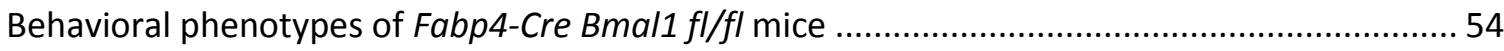

Fabp4-cre Bmal1 fl/fl mice show clock disruption in adipose tissue ............................................. 55

Fabp4-cre Bmal1 fl/fl mice show reduced lipolysis and low FFA concentrations in blood........... 57

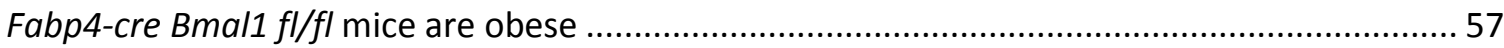

Fabp4-cre Bmal1 fl/fl mice exhibit a metabolic shift to carbohydrate utilization ........................ 59

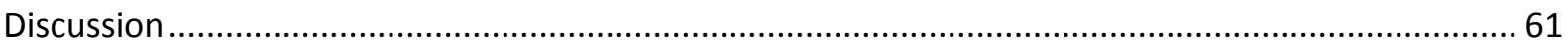

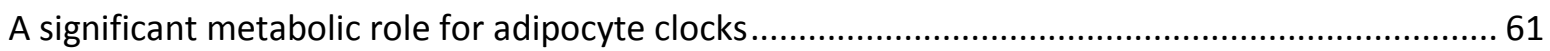

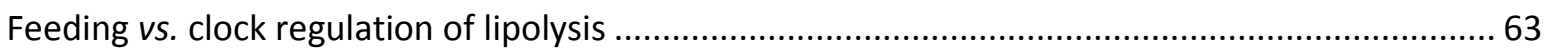




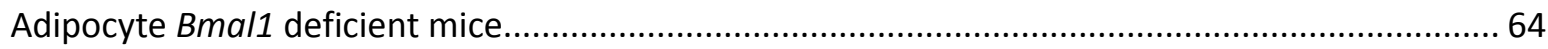

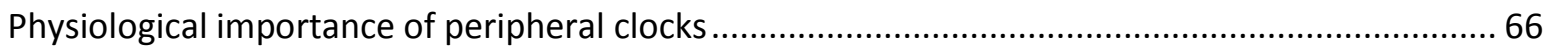

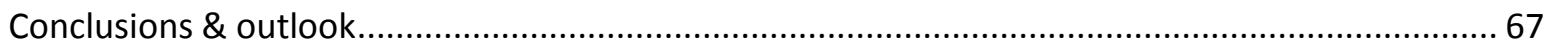

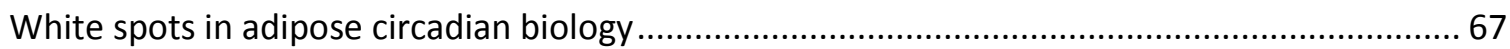

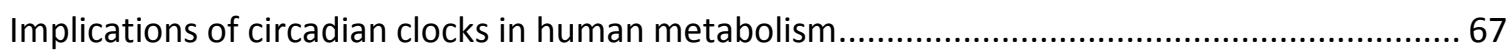

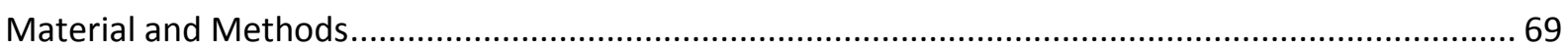

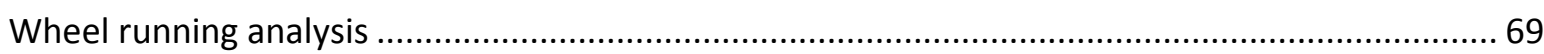

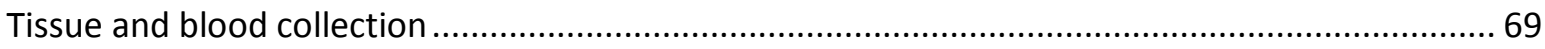

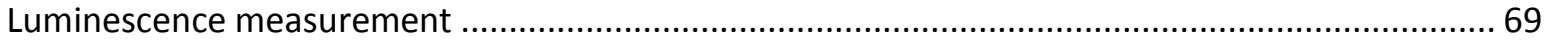

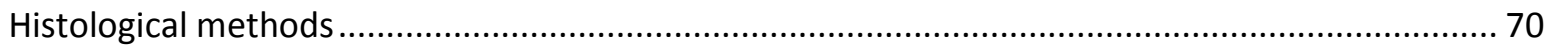

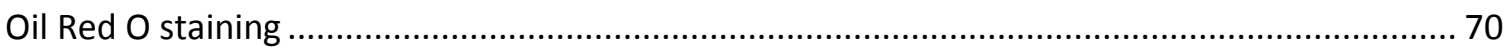

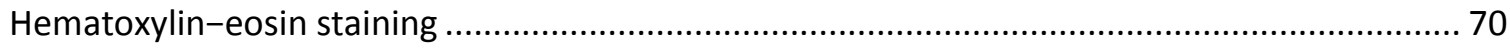

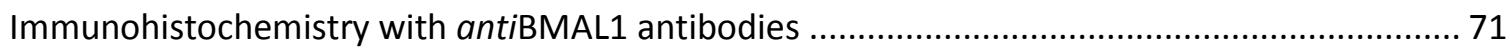

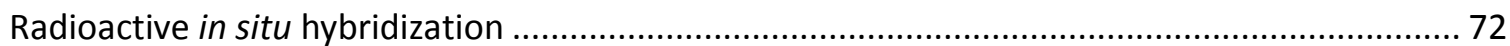

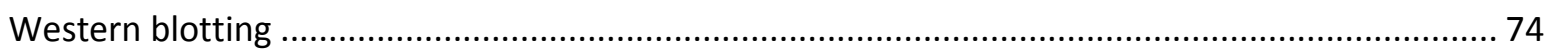

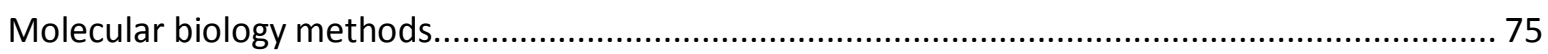

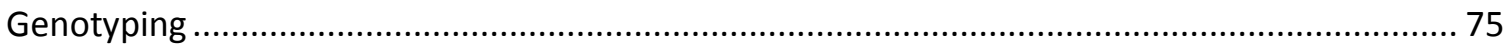

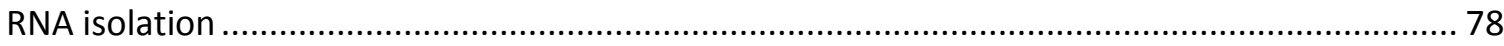

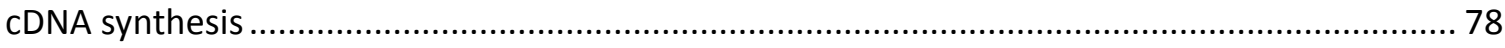

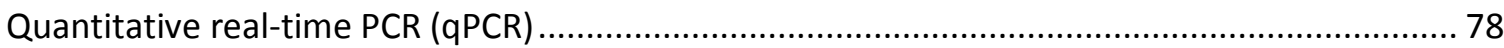

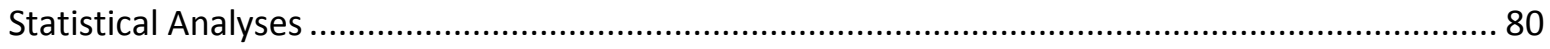

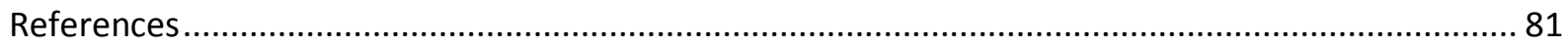

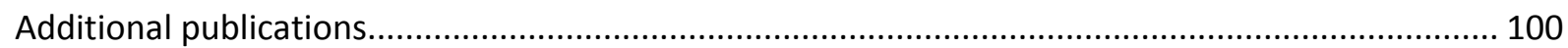

Publication: Diurnal rhythm of circulating Nicotinamide Phosphoribosyltransferase

(Nampt/Visfatin/PBEF): impact of sleep loss and relation to glucose metabolism ....................... 100

Publication: Synaptotagmin10-Cre, a driver to disrupt clock genes in the SCN ........................... 106

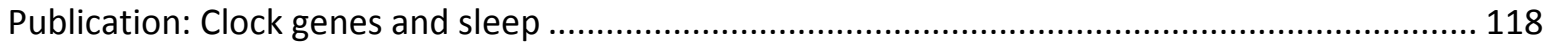

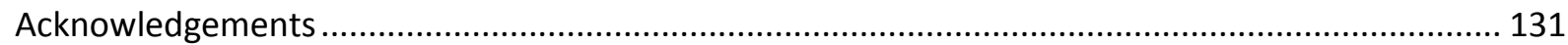

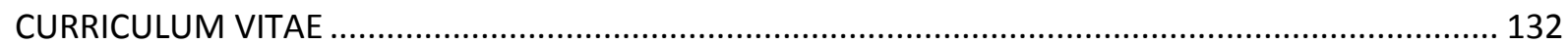




\section{Summary}

The day-night recursion generated by rotation of the Earth around its axis imposes 24 hour rhythm of light and temperature changes on all organisms. To cope with these changes and associated challenges biological species from bacteria to humans developed an adaptational timer - the biological clock. In mammals virtually all cells have a cell-autonomous oscillator consisting of clock genes arranged in transcriptional-translational feedback loops (TTLS). Body array of single-cell clocks is organized in a hierarchical system with the master pacemaker located in the hypothalamus and peripheral clocks found in different organs. This setup facilitates an effective anticipation and synchronization of the physiology to different daily events in order to improve survival. When devoid of any external time information the circadian system is able to generate sustained oscillations in behavioral and physiological processes with an endogenous period length of approximately 24 hours (hence "circa" = approximately and "dies" =day in Latin).

In the first part of the thesis we investigate the circadian biology of the adipose tissue. Using tissue culture explants taken from transgenic mouse with a circadian luciferase reporter we show that adipose tissues from various depots of the body bare a self-sustained clock. We found that Atgl and $H s /$ genes involved in the lipid mobilization (lipolysis) exhibit diurnal variations in the expression which were abrogated in circadian mutant mice. Using cell-based gene reporter techniques and chromatin immunoprecipitation we convincingly demonstrate that Atgl and $\mathrm{Hsl}$ are direct transcriptional targets of the key clock proteins, BMAL1 and CLOCK. In turn this leads to circadian variation in lipolysis efficiency as estimated by glycerol excretion rates from fat pads of wild-type animals. Importantly, circadian changes of lipolysis rate were abolished in adipose tissue of Clock ${ }^{\Delta 19}$ and $\mathrm{Bmal}^{-1}$ mutants. As the result, free fatty acid (FFA) blood content of wild-type animals was rhythmic, unlike in $\mathrm{Clock}^{\Delta 19}$ and Bmal1\% mutants, which had generally low and flat level of FFAs in the blood. As physiological consequence, impaired lipolysis results in decreased availability of FFAs as energy substrate and blunted response to prolonged fasting. On the other hand, lipolysis deficiency triggers accumulation of triglycerides in lipid droplets of adipocytes and thus leads to adiposity and ultimately to obesity.

In the second part we generated a circadian mutant mouse deleting Bmal1 gene in the adipose tissues using the Cre/loxP gene targeting technology. Fabp4-Cre Bmal1 $\mathrm{fl} / \mathrm{fl}$ mice showed impaired expression of Atgl and Hsl, which led to reduction of lipolysis in the adipose tissues accompanied by lower FFA content in the blood. Consistently, Fabp4-Cre Bmal1 fl/fl mutants mostly utilized carbohydrates instead of FFAs during the resting phase. Using this conditional knockout model we 
show that circadian regulation of lipolysis by the adipose clock is also important for body weight control. Indeed, Fabp4-Cre Bmal1 fl/fl mice became heavier than wild-type controls kept on standard diet and developed morbid obesity when fed with high-fat diet.

In summary, we found that the adipose tissue clock is responsible for the regulation of lipid mobilization and their usage as energy source. We conclude that the adipocyte clock is an integral part of the circadian system, which normal functioning is required for metabolic homeostasis 


\section{Introduction}

\section{Biological rhythms}

Live forms on Earth exhibit a large variety of cyclic phenomena known as biological rhythms. These time-dependent variations, occurring in many physiologically important processes, originate predominantly as an adaptation to recurring changes in the external environment. Period spectra of biological rhythms range from seconds (beating of the heart) to years (hibernation) or even decades (for example the population cycle of Magicicada), and often depend on the particular habitat which an individual populates. Therefore the majority of rhythmic processes is distinct among different species and is attributed to particular biological forms.

Circadian rhythms are arguably the most prominent regular biological oscillations. The 24-hour period of day-night changes imposed by the Earth's rotation comprises an extremely universal environmental parameter which creates inevitable daily variations in the availability of numerous natural resources such as light, heat, food etc. Thus, circadian rhythms are one of the most common external conditions shared by all kingdoms of life. As an adaptation to that, organisms developed circadian clocks - an evolutionally promoted internal timekeeping system.

First documented in plants by the French astronomer Jean-Jaques Dortous de Mairan in 1729, circadian clocks are found on almost all levels of the phylogenetic tree from cyanobacteria to modern Homo sapiens. Given such striking conservation, the question as to what evolutionary advantages it confers becomes extremely interesting. There are several hypotheses which provide an adequate explanation. It is very likely that ancient unicellular organisms developed circadian clocks to avoid DNA damage induced by UV during the day, thus restricting DNA replication and cell division to the night (Pittendrigh, 1993). Some evidence supports this hypothesis. For instance, CRYPTOCHROMES, circadian clock proteins in insects and mammals, are light sensitive flavoproteins and belong to the family of light-induced DNA repair enzymes - DNA photolyases (Hoang et al., 2008; Lin and Todo, 2005). This "escape-from-light" theory could explain the clock origin in primitive species, whereas more complex multicellular organisms possess a shielding layer - skin - which protects cell proliferation within the body. Yet the circadian clock does not seem to be merely a rudimentary organ. Clocks confer a clear selective advantage and improve the fitness of the organism on different organizational levels. Indeed, mutant Arabidopsis strains flower later under long-day (16 hours of light : 8 hours of dark) conditions and are less viable under very short-day (4 hours of light : 20 hours of dark) conditions than their wild-type counterparts (Green et al., 2002). Tau-hamsters, which show an extremely short circadian period, also have decreased longevity when compared to wild-type 
animals (Hurd and Ralph, 1998). Similar effects are also observed in unicellular organisms. Rhythmic cyanobacterial strains out-compete arrhythmic strains in light/dark conditions, although both show similar growth rates in a constant environment (Woelfle et al., 2004). Furthermore strains with a resonating period (close to that of the light/dark cycle) have a selective advantage over cyanobacteria with diverging periods (Ouyang et al., 1998). Taken together, organisms with impaired clocks lack one important quality - the reliable anticipation of upcoming daily changes. They cannot form proper temporal associations with time of food appearance, predator activity or temperature changes. Not surprisingly all adaptive values of the circadian clock dissipate in constant laboratory conditions or arctic latitudes. In line with this arctic mammals exhibit much less - if any - circadian behavior (Lu et al., 2010; van Oort et al., 2005).

\section{Properties of circadian clocks}

As mentioned previously, many biological reactions are cyclic in nature. However not all of them conform with the classical notion of an "oscillator" since they are simply direct reactions to a repeated stimulus ("masking"). In order to be called a true "clock" the process in question must stay rhythmic or be sustained even in absence of external cues. For instance, light is the main and most accessible source of time information (Zeitgeber) for the circadian system. Nevertheless animals still maintain circadian behavior in constant light conditions. For instance, Jürgen Aschoff demonstrated in his famous bunker experiment that humans kept in isolation from any time information show regular sleep-wake and body temperature rhythms (Aschoff, 1965). Interestingly, under such freerunning conditions individuals express their own genetically programmed internal period (also called $\tau)$, which is not exactly 24 hours. Thus, in order to keep the proper phase relationship with respect to geophysical daytime, the clock needs to be constantly reset or entrained. This allows organisms to adapt their circadian clocks to seasonal and other environmental changes. Apart from light there are other types of (non-photic) Zeitgebers which can entrain circadian clocks, such as temperature (Aschoff and Tokura, 1986; Francis and Coleman, 1997) and food (Honma et al., 1983). Another important aspect of proper clock function - in particular in poikilotherm species - is temperature compensation. Temperature is known to regulate the frequency of many biochemical reactions (Dutt and Muller, 1993). However, during warm or cold exposure circadian clocks show only moderate changes in period (Hastings and Sweeney, 1957; Pittendrigh, 1954). Some theoretical studies suggest a model in which temperature sensitive counteracting reactions equally slowing or accelerating the period upon temperature change annul the period differences (Kurosawa and Iwasa, 2005; Ruoff et al., 1997). In mammals, this process was proposed to be mediated via robust temperature-insensitive phosphorylation of clock proteins, e.g. by casein kinase I (CKI) (Isojima et al., 2009). Although the molecular basis for temperature compensation still remains to be elucidated, the value of this adaptation for the majority of biological species is hard to overestimate. 


\section{Molecular circadian clocks}

In the early 1970s Seymour Benzer and Ronald Konopka provided first genetic evidence for circadian clock function. A direct mutagenesis screen with ethyl methanesulfonate (EMS) allowed them to isolate mutant lines of Drosophila melanogaster, which had altered rhythms of both locomotor activity and eclosion. In their seminal work arrhythmic, short and long period mutations were mapped to one genomic locus named period ( per $^{0}$, per $^{\mathrm{s}}$ and per $^{\prime}$ respectively) (Konopka and Benzer, 1971). Nevertheless it took another 17 years before the first circadian mutant in hamsters (called Tau) was identified, thus pioneering circadian genetics in mammals (Ralph and Menaker, 1988). These findings opened a whole new avenue for behavioral genetics. Soon after, the Clock gene was discovered in both Drosophila (Dushay et al., 1990) and the mouse (Vitaterna et al., 1994). In 1990 Michael Rosbash proposed the transcriptional feedback loop model as the molecular basis of circadian timekeeping which is still conventional to this day (Hardin et al., 1990).

According to this model, the heart of the oscillatory mechanism is comprised of a set of interlocking transcriptional-translational feedback loops (TTLs) composed of bona fide transcription factors conserved across phyla (Figure 1). In the beginning of the day, positive components of the main loop, CLOCK/NPAS2 and BMAL1 (official acronym: ARNTL), heterodimerize and bind short consensus DNA sequences (E-boxes) in the promoters of the negative components Period1, 2, 3 (Per1-3) and Cryptochrome1, 2 (Cry1/2), activating their transcription. Over the course of the day the concentration of PER and CRY heterodimers increases in the cytoplasm and eventually reaches a threshold at which they are translocated into the nucleus to inhibit CLOCK/BMAL1 activity. Thus the loop is completed and new cycle can start again (Zhang and Kay, 2010). The precise timing of this mechanism is tightly controlled via extensive phosphorylation of PERs and CRYs by CKI (Meng et al., 2008) and 5'-adenosine monophosphate-activated protein kinase (AMPK) (Lamia et al., 2009), respectively, eventually leading to their proteasomal degradation. There are additional loops which confer robustness and stability to the system. Genes of the orphan nuclear receptor family Rev-erb $\alpha / B$ and Ror $\alpha$, which are expressed under the control of CLOCK and BMAL1, impinge on the transcription of a large subset of genes in an antagonizing manner, among them Clock and Bmal1 themselves (Preitner et al., 2002; Sato et al., 2004). This loop regulates phasing and amplitude of clock gene expression and is indispensable for rhythmic behavior (Cho et al., 2012). Other members of the basic helix-loop-helix (bHLH) transcription factors family, DEC1 and DEC2, were shown to bind E-boxes and compete with CLOCK/BMAL1, thus modulating their activity during light resetting (Honma et al., 2002; Rossner et al., 2008). There are several output loops which regulate the circadian transcription of downstream genes. The most prominent among them involves two transcription factors, D-site albumin promoter binding protein $(D b p)$ and $E 4 b p 4$. These compete for the binding of another circadian DNA motif, the D-box, on the promoters of some genes (Lopez- 
Molina et al., 1997; Mitsui et al., 2001). This complex system of negative and positive regulators generates robust molecular oscillations of not only clock genes, but hundreds of clock-controlled genes (CCGs), bearing the relevant promoter elements.

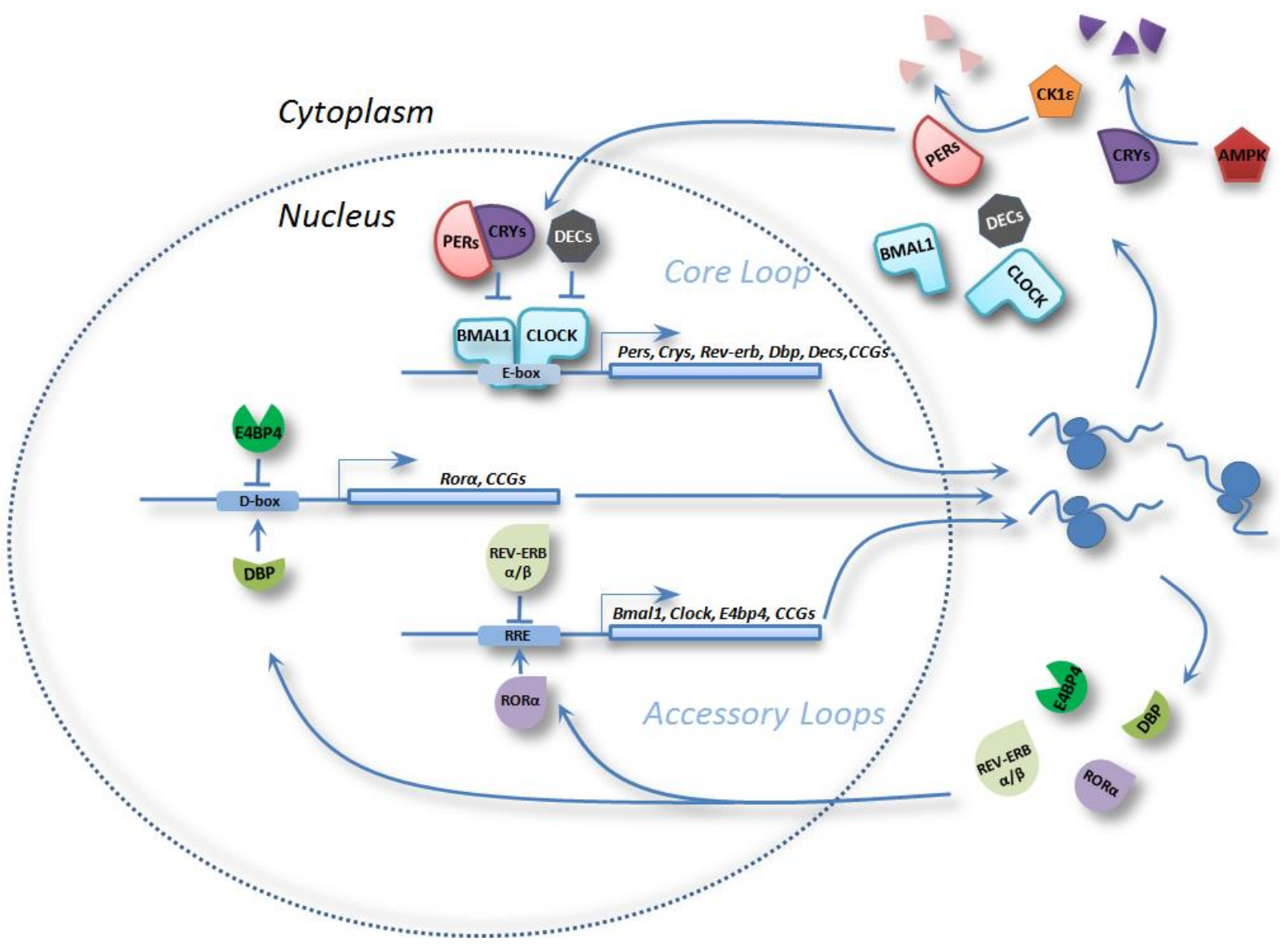

Figure 1. A current model of the circadian transcriptional translational loops (TTLS) in mammals. CLOCK/BMAL1 activate E-boxes in promoters of target genes (Pers, Crys, and CCGs). PER and CRY proteins form a complex which inhibits CLOCK/BMAL1. Additional loops contain Rev-erba/B and Ror $\alpha$ which regulate Clock and Bmal1, and Dbp and E4bp4 which regulate other CCGs. CK1 $1 \varepsilon$ and AMPK phosphorylate PER and CRY proteins, promoting their degradation. For more details see text.

Similar TTLs have been described for invertebrates, plants and fungi, yet they are not the only form of circadian oscillators discovered (Harmer et al., 2001). For instance, Cyanobacteria utilize more simple posttranslational modifications as rhythm generators. This remarkable ancient circadian clock consists of three proteins, KaiA, KaiB and KaiC, which are able to generate circadian rhythms in absence of any transcription (Tomita et al., 2005). Moreover when mixed in vitro, these recombinant proteins are able to reconstitute temperature compensated circadian oscillations of KaiC phosphorylation states (Nakajima et al., 2005). Nonetheless even this expression-independent clock exploits transcriptional regulation to deliver timing information to its CCGs (Nakahira et al., 2004). The existence of posttranscriptional clocks led to the hypothesis that such oscillators might exist in 
other more complex species. This idea was supported by recent reports of rhythmic peroxiredoxin oxidation in human red blood cells and in the alga Ostreococcus tauri under constant darkness conditions, both of which are devoid of transcription (O'Neill and Reddy, 2011; O'Neill et al., 2011). Peroxiredoxins are anti-oxidant proteins that scavenge reactive oxygen species (ROS) in the cell. It appears that they represent a novel biochemically sensitive timekeeping mechanism found in all major domains of life (Edgar et al., 2012). However, it still remains unclear what the function of a peroxiredoxin clock is, and whether they have any link to the TTL. In particular, such a link becomes interesting since the TTL is surprisingly resilient to the inhibition of global transcription rates (Dibner et al., 2009).

\section{The circadian system of mammals}

\section{The master clock}

To keep the body clock ticking, the circadian system has a hierarchical structure with a central pacemaker at the top. Ablation studies revealed that in mammals this master clock resides in the basal hypothalamus, in the suprachiasmatic nucleus (SCN), and drives locomotor and drinking rhythms (Stephan and Zucker, 1972). Later, in his famous transplantation experiment, Michael Menaker demonstrated that SCN-lesioned animals gain the respective behavioral period properties of mutant SCN grafts (Ralph et al., 1990). Remarkably the SCN is situated directly above the retinohypothalamic tract (RHT) and is directly innervated by the optic nerves (Figure 2) (Levine et al., 1991). The major subset of retina cells which send projections to the SCN are intrinsically photosensitive retinal ganglion cells (ipRGCs), which express the light pigment melanopsin and constitute a non-image forming photosensitive system regulating circadian timing (Berson et al., 2002). Additionally they can integrate light information from rods and cones thus making both systems functionally redundant. Genetic disruption of all three light-sensitive receptors (e.g. melanopsin, rod-opsin and cone-opsin) or chemical ablation of ipRGCs leads to "circadian blindness", a condition when the animal is incapable of entraining its behavior to the external light-dark rhythm (Guler et al., 2008; Hattar et al., 2003). Upon light stimulation of the retina, RHT termini release the neurotransmitters glutamate (Glu) and pituitary adenylate cyclase-activating protein (PACAP) which signal to the SCN. In turn, this activates signaling cascades and the phosphorylation of CREB, which can activate the transcription of Pers leading to phase-shifts of the molecular clockwork (reviewed in (Golombek and Rosenstein, 2010)). The SCN also can acquire non-photic entrainment signals via neuropeptide $\mathrm{Y}$ (NPY) and gamma-aminobutyric acid (GABA) containing neurons from the thalamic intergeniculate leaflet (IGL) and serotonergic termini from raphe nuclei. This combination of photic and non-photic signals leads to a more differentiated response of the SCN to light (reviewed in (Dibner et al., 2010)). 
The function of the master pacemaker is to coordinate other rhythms throughout the brain and the rest of the body. This is achieved via diverse SCN output signals such as sympathetic neuronal connections and hormones (Balsalobre et al., 2000; Vujovic et al., 2008). The SCN sends efferent GABAergic and glutamatergic projections to hypothalamic and thalamic areas (Hermes et al., 1996).

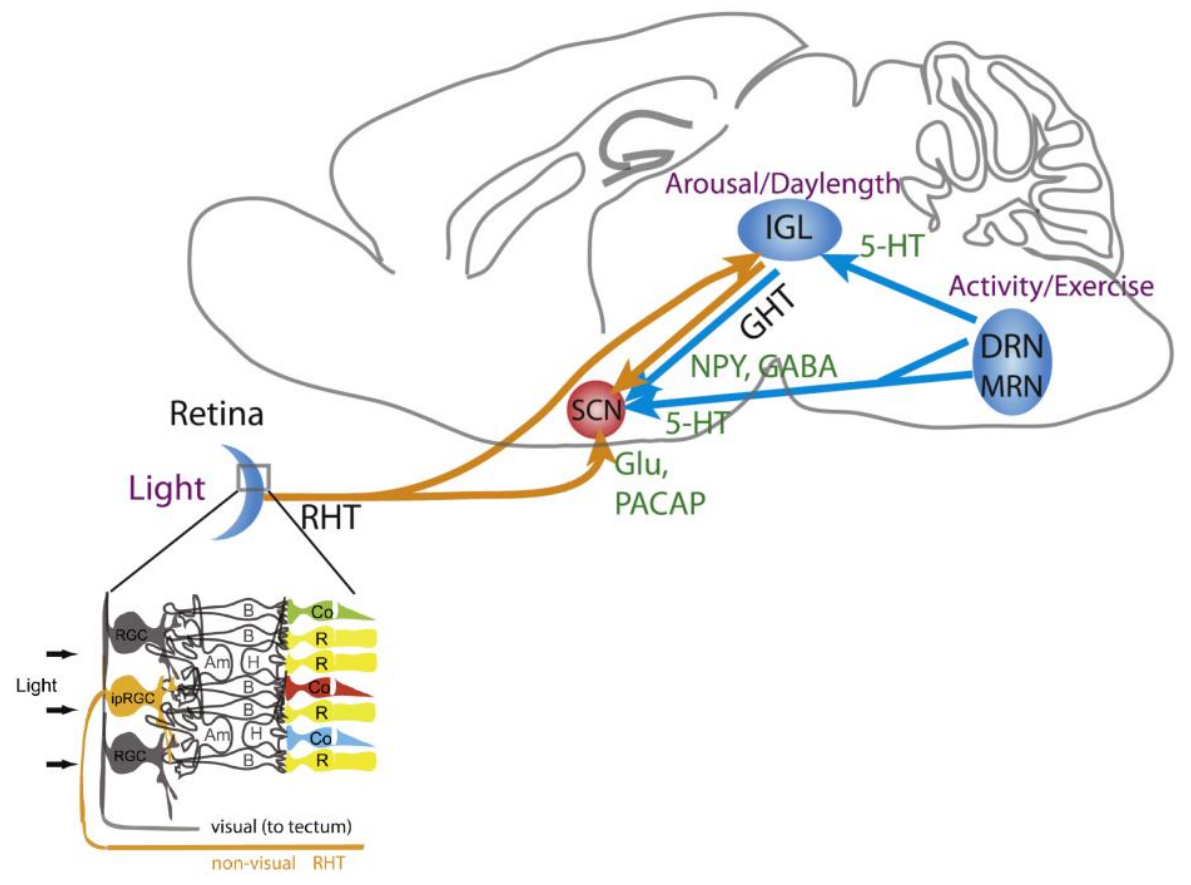

Figure 2. Schematic representation of photic (orange arrows) and non-photic (blue arrows) inputs to the SCN. Intrinsically photosensitive retinal ganglion cells (ipRGCs) project to the SCN via the retinohypothalamic tract (RHT) to transmit light information. (Rods (R); cones (Co); horizontal cells (H); bipolar cells (B); amacrine cells $(A m)$ and regular retinal ganglion cells (RGC); $5-H T$, serotonin; MRN and DRN, dorsal and median raphe nuclei; IGL, intergeniculate leaflet; GABA, gamma-aminobutyric acid; GHT, geniculohypothalamic tract; Glu, glutamate; NPY, neuropeptide Y; PACAP, pituitary adenylate cyclase-activating peptide; RHT, retinohypothalamic tract; SCN, suprachiasmatic nuclei). Modified from (Albrecht, 2012).

In contrast, behavior is primarily regulated by $\mathrm{SCN}$-secreted neuropeptides and thus does not require neuronal connections. Among those SCN factors which may regulate behavioral rhythms are TGF $\alpha$ (Kramer et al., 2001), prokineticin-2 (Cheng et al., 2002), and cardiotrophin-like cytokine (CLC)(Kraves and Weitz, 2006). Furthermore the master clock can affect the synchrony among peripheral organs less directly through the circadian modulation of body temperature (Brown et al., 2002) and feeding behavior (Stokkan et al., 2001).

\section{Peripheral oscillators}

Ubiquitous expression patterns of clock genes indicate that peripheral tissues also contain a circadian clock (Sun et al., 1997; Tei et al., 1997). Indeed many organs show circadian expression of clock genes in vivo (Yamamoto et al., 2004). Furthermore peripheral clocks seem to rely on a similar molecular oscillator mechanism as the SCN (Yagita et al., 2001). Experiments with cell lines gave the first 
indication that non-SCN cells also can sustain circadian expression of clock genes ex vivo (Balsalobre et al., 1998). Subsequently, the development of transgenic animals bearing a circadian promoter coupled to a luciferase reporter helped to identify sustained rhythms in many tissues such as liver, lung, kidney, skeletal muscle, pancreas, heart, stomach, spleen, lung, cornea, thyroid and adrenal glands (Yamazaki et al., 2000; Yoo et al., 2004). Nevertheless, in contrast to the SCN clock where neurons actively use coupling to maintain phase coherence (Liu et al., 2007), peripheral oscillators appear to be less robust and become desynchronized over time (Nagoshi et al., 2004). This evidence led to the re-evaluation of the role of the SCN in circadian regulation. Rather than being a driver of peripheral rhythms, the SCN is now seen as a whole-body synchronizer, which helps to coordinate correct phasing amongst peripheral clocks (reviewed in (Dibner et al., 2010)). This hypothesis is supported by findings that peripheral organs exhibit large internal desynchronization upon SCNlesion in mice (Figure 3) (Yoo et al., 2004).

A

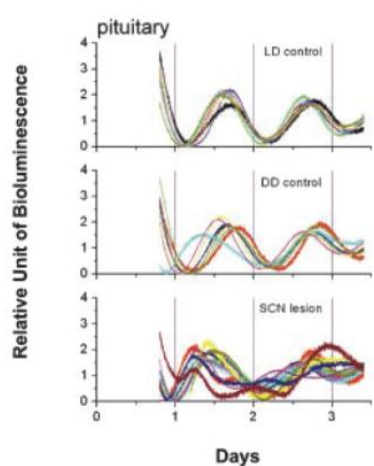

B

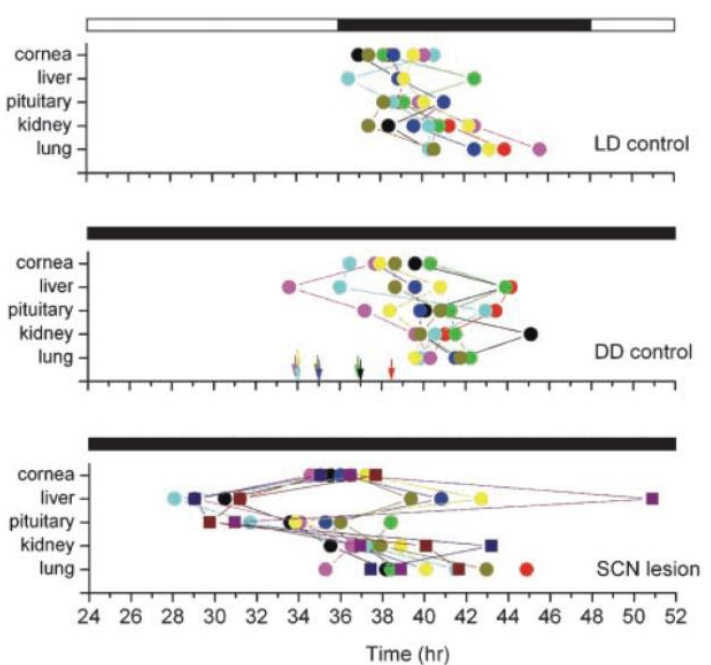

Figure 3. Phases of peripheral oscillators in SCN-lesioned mice. A. Superimposed bioluminescent traces from pituitary and lung slice cultures from $\mathrm{mPer}^{\mathrm{Luc}}{ }^{\mathrm{C}}$ control and SCN-lesioned mice kept in LD and DD. B. Phase map of different clocks in $m P e r 2^{L u c}$ control and SCN-lesioned mice. Modified from (Yoo et al., 2004).

It is important to note that other cues can take over this SCN function. For instance, timing of food intake can uncouple peripheral clocks from SCN control. Upon restricted feeding (RF), when food is provided only during the rest period, the phase relationship between the central clock and peripheral clocks is inversed (Damiola et al., 2000; Stokkan et al., 2001).

\section{Circadian clock and metabolism}

Day-night variations in food consumption, activity and rest imply daily changes in the body's energy state. Indeed, accumulating evidence indicates that circadian rhythms and metabolism are tightly 
interconnected. In rodents, many hormones and metabolites responsible for energy homeostasis have been shown to exhibit circadian oscillation, such as insulin, glucose (La Fleur et al., 1999), corticosterone (De Boer and Van der Gugten, 1987), leptin (Ahima et al., 1998) and triglycerides (Rudic et al., 2004). Interestingly, circadian rhythmicity of some of these blood parameters is eliminated upon SCN ablation, suggesting a role for the master pacemaker in metabolic control (Kalsbeek et al., 2001; La Fleur et al., 1999). Recent studies demonstrate that circadian disruption increases the risk of metabolic disorders. Human night shift workers show a higher prevalence of metabolic syndrome and cardiovascular diseases (De Bacquer et al., 2009; Karlsson et al., 2001; Pan et al., 2011). These epidemiological findings have been replicated under more controlled laboratory conditions. Human individuals subjected to a forced desynchrony paradigm, dissociating behavioral rhythms from the circadian system (achieved by enforcing 28-hour periods of sleep-wake and eating cycles), exhibit hyperglycemia, hyperinsulinemia and hypoleptinemia (Scheer et al., 2009). Moreover, mice subjected to light exposure during the night or fed exclusively during their rest phase show increased body weight (Arble et al., 2009; Fonken et al., 2010). Vice versa, metabolic disturbances can impinge on the circadian system. Some clock genes exhibit altered expression patterns in the peripheral organs of streptozotocin-induced diabetic animals (Herichova et al., 2005; Oishi et al., 2004). Moreover genetically obese KK mice and obese diabetic KK-Ay mice show suppressed expression of clock and adipokine genes in adipose tissues compared to wild-type animals (Ando et al., 2006). Strikingly, high-fat diet in itself can ameliorate behavioral and molecular rhythms in wildtype mice (Kohsaka et al., 2007). Tight coupling of the core clock machinery to the physiological system occurs already on the cellular level (Bass and Takahashi, 2010). AMPK, an intracellular sensor of AMP/ATP ratios, promotes degradation of CRY1 upon high AMP levels and thus feeds back to the TTL (Lamia et al., 2009). Another pathway involves the NAD+-dependent deacetylase SIRTUIN 1 (SIRT1) which is present in CLOCK/BMAL1 complexes and modulates their transcriptional activity (Asher et al., 2008; Nakahata et al., 2008). The circadian clock elicits a feedback on SIRT1 through transcriptional control of nicotinamide phosphoribosyltransferase (Nampt), the main enzyme of $\mathrm{NAD}^{+}$ regeneration passage (Nakahata et al., 2009; Ramsey et al., 2009). These fuel-sensing pathways can modulate clock function and thus couple circadian rhythms to the nutritional state of the cell. In summary, evidence obtained in these studies underpins the intimate relationship between circadian rhythms and metabolism on different organizational levels.

\section{Metabolic phenotypes of circadian clock mutant mice}

Genetic models of circadian disruption in mice provide new tools to study interactions of circadian and metabolic systems. Targeted deletion or mutation of individual clock components leads to metabolic abnormalities, as illustrated by examples discussed below and in Table 1. 


\section{Clock}

In 2005 Turek et. al. in their seminal work demonstrated that mice carrying a non-sense mutation in exon 18 of the Clock gene develop obesity and metabolic syndrome (Turek et al., 2005). These animals become arrhythmic when placed in constant darkness (DD) due to the translation of a dominant negative form of the CLOCK protein which still heterodimerizes with BMAL1, but lacks transactivation activity (Gekakis et al., 1998; Katada and Sassone-Corsi, 2010; Vitaterna et al., 1994). Under entrained light-dark (LD) conditions $\mathrm{Clock}^{\Delta 19}$ mice show disrupted feeding rhythms and hyperphagy which leads to elevated lipids and glucose in the blood, increased adiposity and hepatic steatosis. In addition, both orexin and ghrelin transcript levels, neuropeptides regulating food intake, are reduced in these animals (Turek et al., 2005). Surprisingly Clock ${ }^{-/}$mice, which have a very mild behavioral phenotype, still exhibit elevated body weight gain, although their food intake remains normal (Debruyne et al., 2006; Eckel-Mahan et al., 2012).

\section{Bmal1}

Disruption of Bmal1 results in complete behavioral arrhythmicity in DD (Bunger et al., 2000). At the physiological level this leads to loss of oscillations in glucose and triglycerides in the blood (Rudic et al., 2004). Moreover Bmal1 ${ }^{-1}$ animals suffer from glucose intolerance and increased insulin sensitivity due to low insulin levels (Lamia et al., 2008; Rudic et al., 2004). As a result, when kept on a high-fat diet $\mathrm{Bmal1}^{-}$mice become obese and show increased fat content when compared to wild-type animals, albeit this result was not obtained by another study (Guo et al., 2012; Hemmeryckx et al., 2011; Shimba et al., 2011). Of note, Bmal1 mutants have reduced lifespans and after several months succumb to a premature aging phenotype, which does not allow to study their long-lasting metabolic defects (Kondratov et al., 2006).

\section{Periods}

As a member of the negative feedback loop, Per2 is important for the generation of rhythmic locomotor behavior in DD (Zheng et al., 1999). Consistent with this Per2 ${ }^{-/-}$mice show no discernible circadian corticosterone rhythm, although the glucocorticoid response to stress is intact (Yang et al., 2009). On standard diet $\mathrm{Per}^{-/-}$mice weigh slightly less than their wild-type controls, although overall food consumption remains similar (Grimaldi et al., 2010). In contrast, when challenged with high-fat diet, $\mathrm{Per}^{-/-}$animals become hyperphagic and gain more weight than wild-types. Moreover Per1/2/3 triple-deficient mice are also overweight compared to wild-type controls on high-fat diet (Dallmann and Weaver, 2010) . 


\begin{tabular}{|c|c|c|}
\hline Clock gene & TTL Function & Metabolic defects in mutant mice \\
\hline Clock & $\begin{array}{l}\text { bHLH-PAS domain containing } \\
\text { transcription factor, positive } \\
\text { regulator }\end{array}$ & $\begin{array}{l}\text { - Metabolic syndrome (in Clock }{ }^{\Delta 19} \text { mice)(Turek et al., } \\
\text { - Higher body weight, reduced arterial blood pressure } \\
\text { and altered renal function (in Clock }{ }^{-/} \text {mice) (Eckel- } \\
\text { Mahan et al., 2012; Zuber et al., 2009) }\end{array}$ \\
\hline Bmal1 & $\begin{array}{l}\text { bHLH-PAS domain containing } \\
\text { transcription factor, positive } \\
\text { regulator }\end{array}$ & $\begin{array}{l}\text { - Increased adiposity, abolished oscillations in plasma } \\
\text { glucose and triglycerides and premature aging (in } \\
\text { Bmal1 }{ }^{-1} \text { ) (Guo et al., 2012; Kondratov et al., 2006; } \\
\text { Rudic et al., 2004) } \\
\text { - Fasting hypoglycemia (in liver-specific KO) (Lamia et } \\
\text { al., 2008) } \\
\text { - Diabetes mellitus (in pancreas-specific KO) } \\
\text { (Marcheva et al., 2010; Sadacca et al., 2011) }\end{array}$ \\
\hline Per1 & $\begin{array}{l}\text { PAS-domain containing negative } \\
\text { regulator }\end{array}$ & $\begin{array}{l}\text { - Increased urinary sodium excretion (Gumz et al., } \\
\text { 2009) } \\
\text { - Lower blood pressure (Stow et al., 2012) }\end{array}$ \\
\hline Per2 & $\begin{array}{l}\text { PAS-domain containing negative } \\
\text { regulator }\end{array}$ & $\begin{array}{l}\text { - Altered lipid metabolism, lower body weight } \\
\text { (Grimaldi et al., 2010) } \\
\text { - Higher body weight and adiposity on high-fat diet } \\
\text { (Yang et al., 2009) }\end{array}$ \\
\hline Cry1, Cry2 & Negative regulator & $\begin{array}{l}\text { - Reduced size and body weight (Bur et al., 2009) } \\
\text { - Hyperglycemia and glucose intolerance (Lamia et al., } \\
\text { 2011; Zhang et al., 2010) } \\
\text { - Salt-sensitive hypertension (Doi et al., 2010) }\end{array}$ \\
\hline Rev-erbo & $\begin{array}{l}\text { Nuclear receptor, negative } \\
\text { regulator }\end{array}$ & $\begin{array}{l}\text { - Increased body weight and adiposity on high-fat diet } \\
\text { (Delezie et al., 2012) } \\
\text { - Increased serum triglycerides (Raspe et al., 2002) }\end{array}$ \\
\hline Ror $\alpha$ & $\begin{array}{l}\text { Nuclear receptor, positive } \\
\text { regulator }\end{array}$ & $\begin{array}{l}\text { - Reduced plasma triglycerides and HDL, enhanced } \\
\text { atherosclerosis (Mamontova et al., 1998) }\end{array}$ \\
\hline
\end{tabular}

Table 1. Metabolic defects in mice harboring mutations in clock genes. Modified from (Sahar and SassoneCorsi, 2012).

\section{Cryptochromes}

Other important members of the TTL's negative loop are the Cryptochrome genes. Mice lacking both Cry genes are arrhythmic in constant darkness and are frequently used as general clock-deficient model (van der Horst et al., 1999). Not surprisingly, Cry $1^{--}$Cry $2^{-/}$mutants also exhibit a large variety of metabolic disturbances. Cry-deficient animals exhibit a marked reduction in size and body weight compared with controls (Bur et al., 2009). Nevertheless, Cry $1^{-} \mathrm{Cry}^{-1}$ mice show perturbed sugar metabolism with hyperglycemia and glucose intolerance, hallmarks of diabetes (Lamia et al., 2011). Although blood triglyceride levels of Cry-deficient mice are reduced, they still develop hepatic steatosis indicating defects in triglyceride metabolism (Cretenet et al., 2010). 


\section{Nuclear receptors}

Initially thought to be merely clock modifiers, the members of the nuclear receptor family Rev-erb $\alpha$ and $b$ are now accepted as bona fide components of the core clock. Loss of both genes in liver strikingly affects the hepatic circadian transcriptome, with particular emphasis on clock and metabolic function (Cho et al., 2012). Behaviorally arrhythmic Rev-erb double knockout mice display a shift of overall body metabolism to a more oxidative state, with increased blood glucose and triglyceride levels (Cho et al., 2012). Furthermore, circadian nuclear receptors become an enticing therapeutic target since the availability of both potent Rev-erb agonists and antagonists provides a new pharmacological approach to reset a disrupted metabolic balance (Kojetin et al., 2011; Kumar et al., 2010; Solt et al., 2012).

\section{Peripheral clocks as metabolic regulators}

Many physiological processes which take place in peripheral organs show circadian oscillations. Among them are xenobiotic detoxification (Gachon et al., 2006), sugar and lipid metabolism (Rudic et al., 2004), and blood pressure and pulse rates (Veerman et al., 1995). According to genome-wide transcriptome profiling studies up to $12 \%$ of all genes in peripheral tissues display daily oscillations in their expression (so called CCGs). These include genes encoding rate-limiting enzymes and critical regulators of many metabolic pathways, thus providing a link between the local circadian system and metabolism (Panda et al., 2002; Storch et al., 2002). Moreover, many CCGs are transcription factors that constitute a physiological output of the circadian clock. Krüppel-like factors KIf10 (Guillaumond et al., 2010) and KIf15 (Jeyaraj et al., 2012a; Jeyaraj et al., 2012b) and orphan nuclear receptors (Yang et al., 2006) are regulated by clock genes, and in turn convey timing information to their downstream targets. Therefore the function of peripheral clocks - and in particular their metabolic applications represents an interesting objective to study. Employing an transplantation model, Oster and colleagues showed that the circadian clock in the adrenal gland regulates adrenal sensitivity to ACTH, and is required for normal corticosterone rhythms in mice (Oster et al., 2006). Disrupting the essential clock gene, Bmal1, in the liver with the Cre-loxP recombination strategy, Lamia et. al. demonstrated the physiological significance of the hepatic oscillator. Their experiments suggest that the liver clock contributes to blood sugar homeostasis by driving the circadian export of glucose via transcriptional control of glucose transporter 2 (Glut2, S/c2a2). The peak of Glut2 expression coincides with the fasting phase, thereby favoring glucose export into the circulation when no food is ingested (Lamia et al., 2008). Marcheva et. al. established a role for the pancreatic clock in the regulation of glucose metabolism. Islets devoid of a $\beta$-cell clock become refractory to glucose and are incapable of secreting insulin, which eventually leads to the development of a diabetic state (Marcheva et al., 2010). 


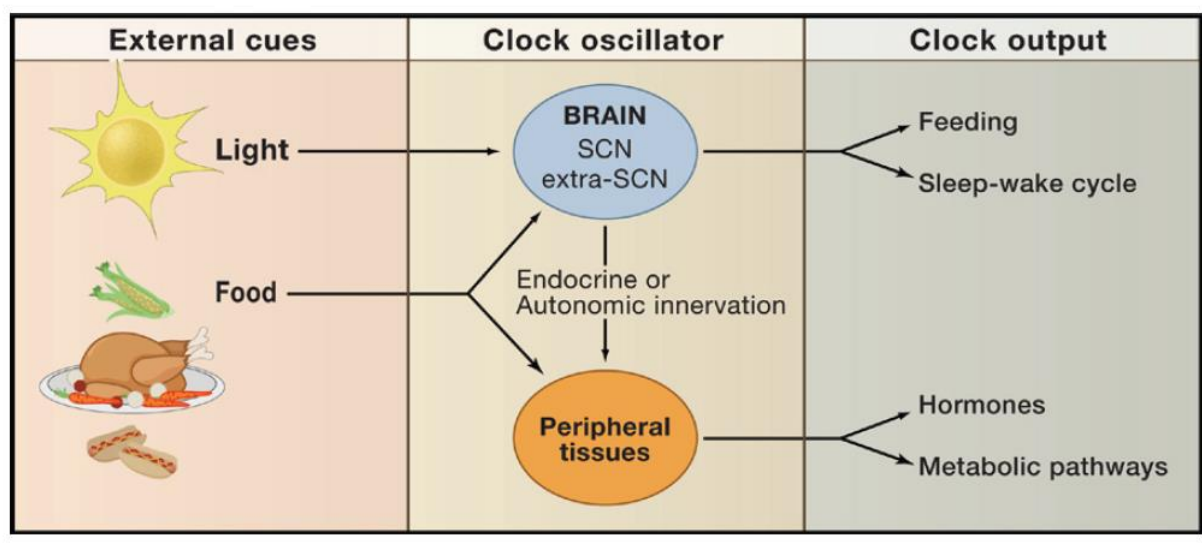

Figure 4. Simplified representations of external zeitgebers and clock physiological outputs. Light is the predominant external cue for the $\mathrm{SCN}$, whereas other clocks in the brain and peripheral tissues can also be entrained by food. The SCN and peripheral clocks subsequently regulate behavior, feeding, the sleep-wake cycle, hormone secretion and metabolic homeostasis. Modified from (Green et al., 2008).

\section{Adipose tissue}

Traditionally adipose tissue is recognized as the main energy storage compartment of the body. It is well established that there are two functionally non-redundant types of adipose tissues, although they both deal with energetically rich lipids as their major substrate. The first type is brown fat, which is specialized for the production of heat by non-shivering thermogenesis utilizing lipids as a fuel. In contrast, white adipose tissue (WAT) stores lipids in the form of triglycerides, and therefore serves as a long-term fuel reservoir. While having considerably higher energetic density than carbohydrates, triglycerides require far less water and are associated in the form of anhydrous lipids droplets. Therefore, the conversion of nutrient excess to triglycerides makes the process of energy buffering more convenient. It is worthwhile to mention that subcutaneous WAT also acts as a heat insulator owing to its low thermal conductivity. Moreover, due to its mechanical properties and body distribution, WAT provides additional cushioning and thus protects internal organs from mechanical damage. On the cellular level, mature adipocytes represent the functional unit of adipose tissue, and thus constitute the dominant cell population. Nonetheless a certain fraction of WAT is also comprised of other cell types (fibroblasts, macrophages etc.)(Trayhurn, 2007).

With the increasing prevalence of metabolic disorders and obesity in the modern population, adipose tissue biology attracts rising research interest. Firstly, obesity is characterized by increasing amounts and distribution of fat deposits in the body and therefore adipocytes must be in the focus of our attention. Secondly, adipose tissue is also an important endocrine and signaling organ (Cook et al., 1987). 


\section{Adipokines}

The term adipokine was coined to define cytokine-like proteins secreted from adipose tissue and encompasses large spectra of functionally different molecules. Adipokines comprise known inflammatory cytokines and members of the complement system (e.g. TNF $\alpha$, IL-6, adipsin) as well as appetite regulators (e.g. leptin). With the discovery of leptin in 1994, a pivotal role for adipose endocrine function became apparent (Zhang et al., 1994) . Mice mutant for the leptin gene $o b$ and its receptor $d b$, were first reported in 1950 and 1966 respectively. These mice display morbid obesity (Hummel et al., 1966; Ingalls et al., 1950). Later it was determined that leptin is secreted from adipose tissue and signals to the hypothalamus and periphery for the regulation of food intake and energy balance (Friedman and Halaas, 1998). Considerable scientific attention has been directed toward another adipokine - adiponectin. Independently characterized by four groups, adiponectin is specifically expressed by mature adipocytes and circulates at high levels in the blood (Chandran et al., 2003; Hu et al., 1996; Maeda et al., 1996; Nakano et al., 1996; Scherer et al., 1995). Serum concentrations negatively correlate with adiposity in humans, and lower levels predict an increased risk of diabetes and cardiovascular disease (Arita et al., 1999; Trujillo and Scherer, 2005). Furthermore, studies performed over the last decade suggest that adiponectin exerts anti-diabetic, anti-inflammatory, and anti-atherogenic actions (Wozniak et al., 2009). Another prominent adipokine is visfatin which was initially identified as pre-B-cell colony-enhancing factor 1 (PBEF1) and has nicotinamide phosphoribosyltransferase activity (Rongvaux et al., 2002; Samal et al., 1994). Although it is not clear what the biological action of visfatin is, plasma visfatin levels in humans correlate with obesity and type 2 diabetes (Chen et al., 2006; Haider et al., 2006).

\section{Metabolism of fatty acids}

As was previously mentioned, the vast majority of the body's lipids are found stored in adipose tissue depots as triglycerides. Fat contains more than twice as many calories per unit of mass as protein and carbohydrates, making it an attractive substrate for energy storage. Chemically, triglycerides represent esters of one glycerol molecule and three fatty acid molecules with polar "heads" and hydrophobic "tails". Within adipocytes neutral lipids are organized in large lipid droplets that comprise a hydrophobic core of triglycerides and sterol esters surrounded by a monolayer of phospholipids. Triglycerides themselves, however, do not have energetic value, but only their breakdown products fatty acids and glycerol. In order to be metabolized, triglycerides must undergo lipolysis - a process of ester bond hydrolysis into free fatty acids (FFAs) and glycerol residues. Subsequently, both FFAs and glycerol are exported into the circulation where they are transported to the liver and other organs (summarized in Figure 5). Besides being energy substrates, FFA residues are precursors for lipid and membrane biosynthesis, or important mediators in cell signaling processes. Inside the cell the primary site of FFA metabolism is the mitochondrial matrix where FFAs 
are subjected to $\beta$-oxidation. During this process long carbon chains of fatty acids are split into acetyl-CoA, which eventually enters the Krebs cycle to produce ATP. In the case of extensive $\beta$ oxidation (e.g. during starvation), excessive acetyl-CoA amounts can be utilized via ketogenesis to produce ketones (ketone bodies) that serve as an alternative energy substrate (Hasselbalch et al., 1994).

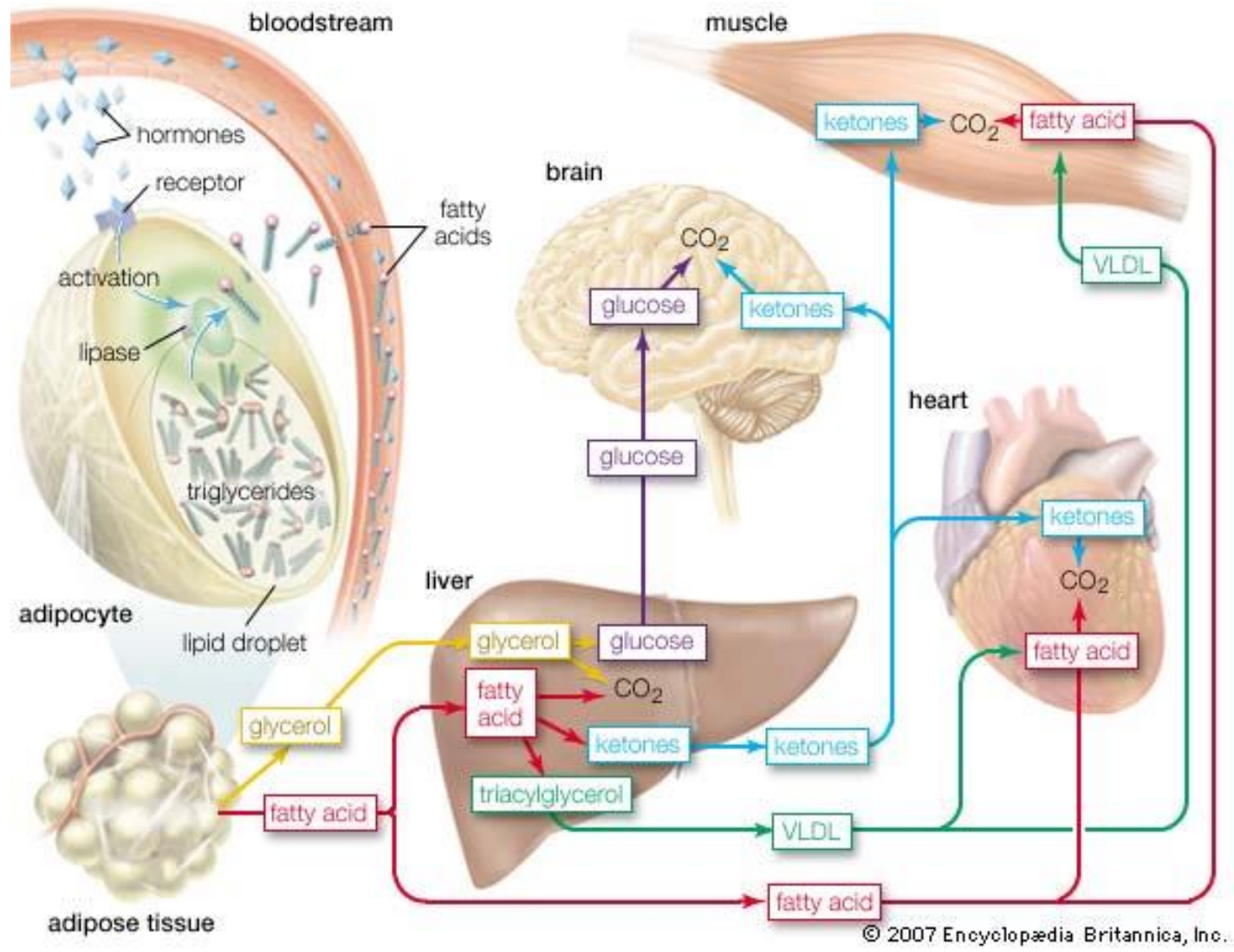

Figure 5. Upon hormonal stimulation adipocyte triglycerides are hydrolyzed (lipolysis) into free fatty acids and glycerol which are released into the bloodstream (left). With the circulation they reach peripheral organs where, in turn, they can be transformed into other energetic compounds (right) (very low-density lipoproteins (VLDL)). Fatty acid mobilization. Art. Encyclopædia Britannica Online. Web. 4 Oct. 2012. <http://www.britannica.com/EBchecked/media/92256/When-hormones-signal-the-need-for-energy-fattyacids-and>.

Adipocyte-derived FFAs are also important substrates for hepatic synthesis of lipoproteins and very low-density lipoproteins (VLDLs)(Fukuda and Ontko, 1984). Glycerol can enter either gluconeogenesis or glycolysis to produce glucose or pyruvate respectively. In summary, excess food-derived FFAs are esterified to chemically neutral triglycerides stored in lipid droplets, whereas upon energy demand, triglycerides stores are mobilized by hydrolytic cleavage making FFAs available for $\beta$-oxidation and ATP production. 


\section{Regulation of lipolysis}

The pool of FFAs in the circulation represents a balance of two counter processes - lipolysis of triglycerides and FFA re-esterification (Kalderon et al., 2000). Energy demand during fasting shifts this balance towards lipolysis. As with any biochemical pathway, lipolysis occurs as multistep regulated process with different enzymes acting at each stage. The first step is catalyzed by adipose triglyceride lipase (ATGL), converting triglycerides to diacylglycerols. This is followed by hydrolysis of diacylglycerols to monoacylglycerols catalyzed by hormone sensitive lipase (HSL). The third and final step involves the degradation of monoacylglycerols to glycerol and FFA residues by monoacylglycerol

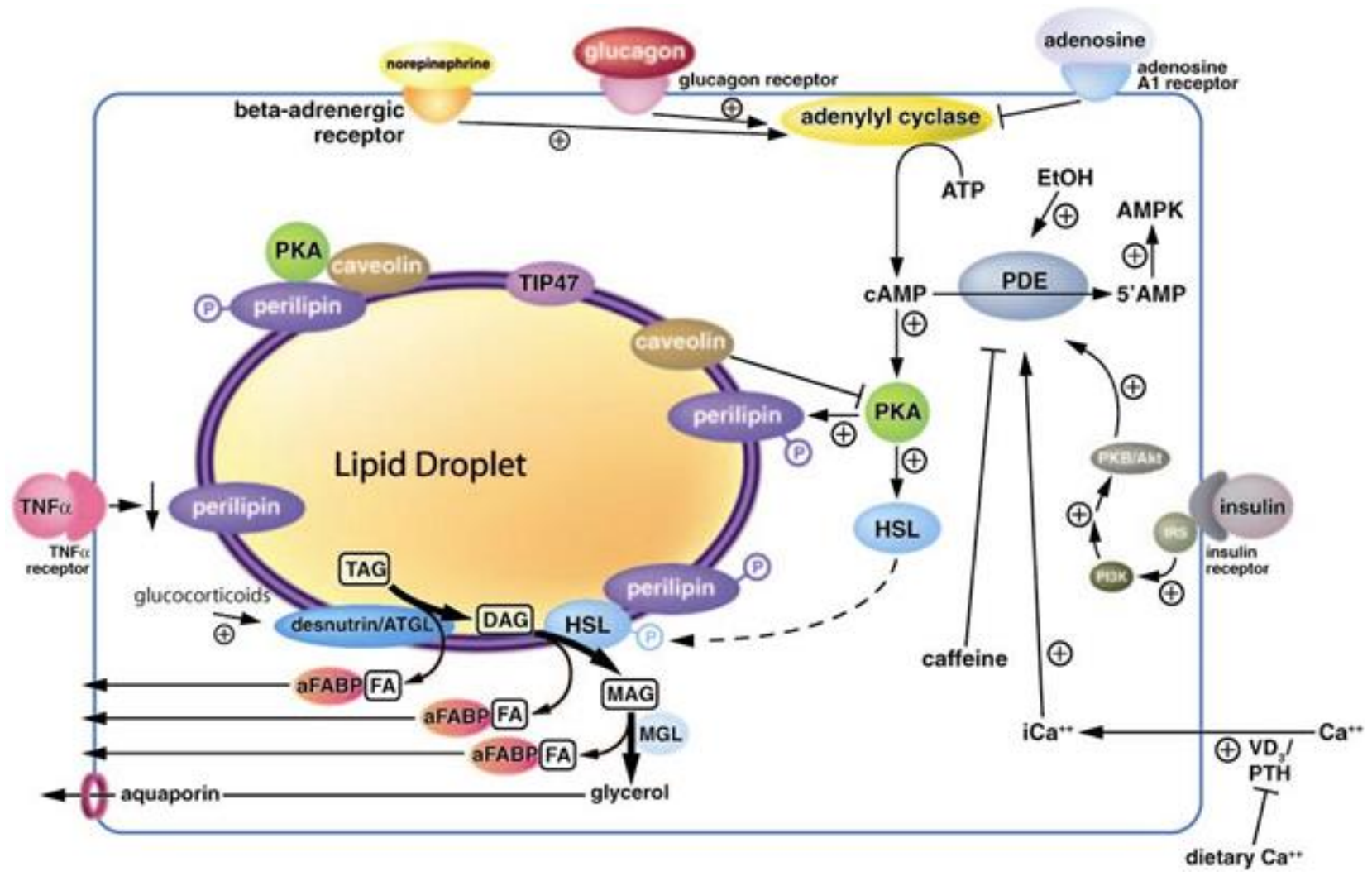

Figure 6. Regulation of adipocyte lipolysis. For details refer to the text (adipocyte fatty acid-binding protein (aFABP); insulin receptor (IR); insulin receptor substrate (IRS); phosphoinositide 3-kinase (PI3K); protein kinase B/AKT (PKB/AKT); protein kinase A (PKA); phosphodiesterase (PDE); hormone sensitive lipase (HSL); adipose triglyceride lipase (ATGL); monoacylglycerol lipase (MGL); triglycerides (TAG); diacylglycerols (DAG); monoacylglycerols (MAG); parathyroid hormone (PTH); vitamin D3 (VD 3 )). Modified from (Duncan et al., 2007).

lipase (MGL) (Duncan et al., 2007; Zechner et al., 2012). Although other lipases may be implicated in lipolysis, ATGL and HSL were reported to facilitate more than $90 \%$ of triglyceride hydrolysis (Schweiger et al., 2006). Activity of these enzymes is controlled by multiple hormonal pathways that can stimulate or inhibit lipolysis (summarized in Figure 6). The most potent lipolysis inducers in adipose tissue are catecholamines that act through $\beta$-adrenergic receptors (Lafontan and Berlan, 1993). When catecholamines bind to their G-protein coupled-receptor, they activate adenylate 
cyclase, thus increasing intracellular cAMP levels. This leads to elevation of cAMP-dependent protein kinase A (PKA) activity. PKA-mediated phosphorylation of lipid droplet-associated proteins - perilipins - and HSL dramatically boosts lipolysis (Holm et al., 2000). In addition to catecholamines, glucagon can also induce lipolysis through adenylate cyclase-dependent PKA activation both in human and mouse adipose tissues (Heckemeyer et al., 1983; Perea et al., 1995). Another positive regulator of lipolysis is TNF $\alpha$. This cytokine downregulates perilipin expression via activation of MAPK p44/42 and JNK (Ryden et al., 2004; Ryden et al., 2002). Caffeine positively regulates lipolysis via inhibition of phosphodiesterase (PDE), preventing the breakdown of cAMP (Acheson et al., 2004; Peers and Davies, 1971). On other hand, adenosine acting through inhibitory $G$ protein-coupled receptors can inactivate adenylate cyclase and reduce lipolysis rates (Borglum et al., 1996; Londos et al., 1978). Insulin has the most potent anti-lipogenic effect. It acts through the insulin receptor (IR) and the subsequent phosphorylation of insulin receptor substrate (IRS). In turn, activation of phosphatidylinositol-3 kinase (PI3K) is triggered, followed by an induction of protein kinase B/AKT (PKB/AKT) which increases PDE activity (reviewed in (Langin, 2006)).

\section{Metabolic consequences of defective lipolysis}

The functional importance of lipid mobilization became evident when genetically deficient mutant mouse models for lipolysis were generated. Surprisingly, $\mathrm{Hs}^{/-}$mice are not overweight, although lipolysis rates and blood FFA content are reduced and adipocyte size is increased (Osuga et al., 2000; Wang et al., 2001). This phenotype is accompanied by extensive accumulation of diacylglycerols in WAT and compensatory decrease in FFA re-esterification and de novo synthesis, which explains the reduction in triglyceride accumulation (Haemmerle et al., 2002; Zimmermann et al., 2003). In contrast, Atgl $^{-1}$ mice display increased adiposity and higher body weight when compared to controls. Moreover, ATGL-deficiency decreases lipolysis rates from WAT and this results in reduced blood FFA levels (Haemmerle et al., 2006). This, again, leads to dramatic ectopic lipid accumulation in organs such as the heart, provoking cardiac failure and premature death (Haemmerle et al., 2006). In line with this, recently described human mutations of the ATGL gene are associated with decreased enzymatic activity and triglyceride accumulation in multiple tissues (Akiyama et al., 2007; Fischer et al., 2007; Kobayashi et al., 2008). The human syndrome of ATGL deficiency was named Neutral Lipid Storage Disease with Myopathy (NLSDM) and is characterized by systemic lipid accumulation, muscular weakness and, in some cases, cardiac abnormalities. Another important consequence of impaired lipolysis is reduced FFA availability in the circulation, which leads to a shift from lipid to carbohydrate usage as an energy substrate. This in turn negatively impinges on various aspects of physiology such as exercise performance, fasting responses and cold exposure tolerance (Ahmadian et al., 2011; Haemmerle et al., 2006; Huijsman et al., 2009; Wu et al., 2012). 


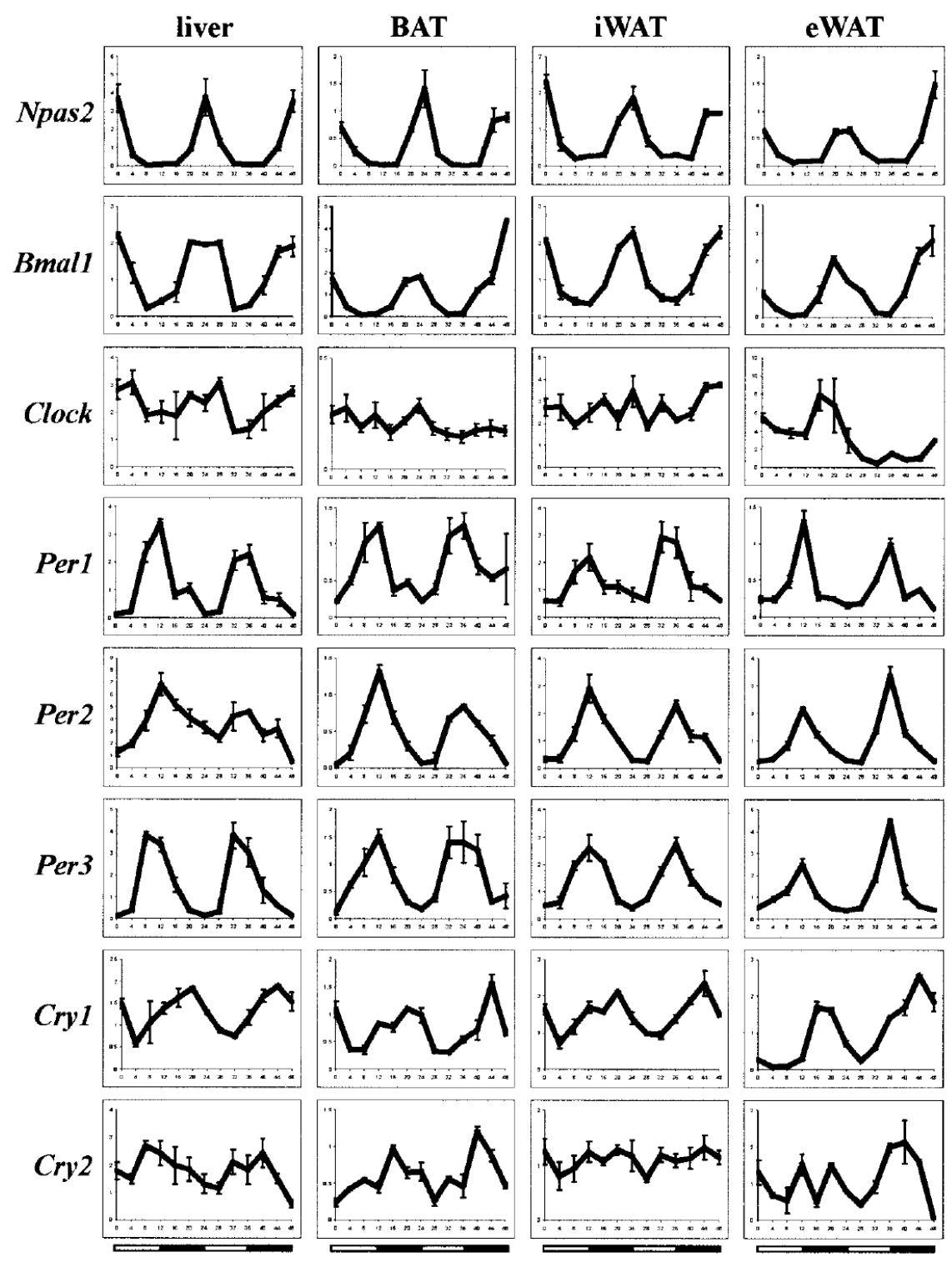

Figure 7. Microarray data demonstrating circadian oscillation of several clock genes in peripheral tissues of wild-type mice under LD conditions for 2 days (brown adipose tissue (BAT), inguinal white adipose tissue (iWAT), epididymal white adipose tissue (eWAT)). White and black bars at the bottom indicate 12 hours of light and 12 hours of dark respectively. Modified from (Zvonic et al., 2006).

\section{The adipocyte circadian clock}

Numerous studies imply tight interconnections between adipocyte physiology and circadian rhythms. In humans, the circulating levels of adipose tissue-derived hormones show diurnal variation, including leptin, adiponectin and visfatin (Benedict et al., 2012; Gavrila et al., 2003; Sinha et al., 1996). Indeed, mRNAs of these adipokines exhibit rhythmic expression, which is attenuated in obese and diabetic animals (Ando et al., 2005). Adipose tissue exhibits rhythmic clock gene expression both in mice and humans, indicating of the presence of peripheral clocks (Ando et al., 2005; Otway et al., 2011; Zvonic et al., 2006) (see also Figure 7). Moreover, according to genome wide microarray profiling data, a large proportion of the WAT transcriptome (up to $20 \%$ ) is subjected to circadian 
regulation, and many of these affected genes perform important metabolic functions (Ptitsyn et al., 2006; Zvonic et al., 2006). Nonetheless, these studies did not address whether this rhythmicity is controlled by a local circadian oscillator or rather is systemically driven via hormonal and/or temperature cues.

Cell based experiments indicate that clock genes are important for adipose tissue function. The expression of Bmal1 is up-regulated during adipogenesis and Bmal1-deficiency perturbs cell differentiation into mature adipocytes (Guo et al., 2012; Shimba et al., 2005). Similar functions were also attributed to Rev-erb $\alpha$, which was reported to play a dual role in adipogenesis. Initially it is required for mitotic cell division and promotes adipocyte differentiation. During later stages, however, Rev-erb $\alpha$ detains triglyceride accumulation via its inhibitory effect on Ppary expression (Wang and Lazar, 2008). Another clock gene, Per2, was shown to modulate adipocyte physiology by interacting with PPARY and preventing its binding to target genes (Grimaldi et al., 2010). 


\section{Aims of the work}

According to the prevailing hypothesis, the central circadian clock located in the SCN controls different aspects of physiology via neuronal and humoral synchronization of peripheral clocks. In turn, peripheral oscillators throughout the body regulate the timing of organ-specific metabolic functions. This is achieved mainly through transcriptional regulation of clock controlled genes involved in metabolism, as it has been demonstrated for the glucose transporter gene Glut2 in the liver (Lamia et al., 2008). White adipose tissue is another essential metabolic organ which regulates energy homeostasis and maintains the balance between consumed and deposited nutrients. Although many aspects of adipocyte biology have been shown to be subjected to 24-hour oscillations, the function of WAT peripheral circadian clocks has not been addressed and remains elusive. We hypothesize that local adipose tissue clocks regulate triglyceride metabolism in order to accumulate energy during periods of food consumption and mobilize it during periods of fasting.

We address this issue by investigating the metabolic defects related to adipose tissue in two previously generated circadian clock mutants, $\mathrm{Clock}^{\Delta 19}$ and Bmal1\% . This approach will help us to identify the circadian component in the physiological processes which underlie triglyceride metabolic regulation. Moreover, to determine the molecular bases of adipose circadian biology, we aim to identify putative clock controlled genes that are responsible for the time-dependent changes in triglyceride metabolism. Further, we will use a genetic approach to disrupt the circadian clock in adipose tissue via deletion of Bmal1 by the Cre/loxP system. Using this animal model in combination with adipose tissue cultures, we will test if WAT clocks are involved in the regulation of energy homeostasis. 


\section{Results}

\section{Circadian regulation of lipid mobilization in white adipose tissues}

In mammals, a network of endogenous circadian clocks regulates 24-hr rhythms of behavior and physiology. Circadian disruption promotes adiposity and the development of obesity-associated disorders such as type 2 diabetes and the metabolic syndrome. While behavioral rhythms are regulated by a central circadian pacemaker, accumulating evidence suggests that peripheral clocks strongly contribute to the regulation of metabolism and, thus, may be involved in the maintenance of energy homeostasis. This study focuses on white adipose tissue (WAT) as the primary energy storage site of the body. We show that local WAT clocks regulate the availability of lipids as energy source via transcriptional regulation of two major lipolysis genes, adipose triglyceride lipase (Atgl or Pnpla2) and hormone-sensitive lipase (Hsl or Lipe). Diurnal variations in lipid mobilization from WAT ensure an increased availability of free fatty acids (FFAs) and glycerol as energy sources during the daily rest period. Circadian Clock and Bmal1 mutant mice show decreased lipolysis rates, correlating with blunted diurnal FFA and glycerol blood profiles and increased adiposity due to lipid accumulation in WAT stores. Our data indicate that local WAT clocks regulate lipid mobilization, ensuring the temporally appropriate availability of lipids as energy source for other tissues. 
Living organisms are influenced by rhythmic changes in a large number of environmental parameters due to the Earth's rotation around its axis. In an attempt to optimally adapt to such recurring events most species have evolved circadian clocks - internal timing systems controlling 24-hr rhythms of behavior and physiology (Harmer et al., 2001). Remarkably, in mammals, most - if not all - cells harbor their own molecular timer. Synchrony amongst these cellular clocks, and, thus, overt behavioral and physiological rhythms, are regulated via a hierarchical system of central and peripheral oscillators. Information about external daytime is perceived by a master pacemaker located in the hypothalamic suprachiasmatic nucleus (SCN) which conveys timing cues to the rest of the body. In both the SCN and the periphery the molecular clock machinery is based on interlocked transcriptional feedback loops comprised of a set of clock genes. The basic helix-loop-helix (bHLH) transcription factors CLOCK and BMAL1 (ARNTL) induce expression of the negative regulators Per1-3 and Cry $1 / 2$ via binding to E-box promoter elements. During the course of the day PER and CRY proteins accumulate in the cytoplasm, then enter the nucleus and repress activity of CLOCK/BMAL1 heterodimers, thereby shutting down their own transcription. Further loops interact with this E-boxmediated transcription rhythm and stabilize its characteristic 24 -hr periodicity. Clock genes further regulate the activity of numerous tissue-specific output genes, thereby translating time-of-day information into physiologically meaningful signals (Storch et al., 2002).

Both rodent and human studies suggest a tight interaction between circadian clock regulation and energy homeostasis. Circadian disruption, either external (as seen for example in shift workers) or internal (e.g. in Clock gene mutant mice), promotes obesity and the development of type 2 diabetes and the metabolic syndrome (Bray and Young, 2007; Rudic et al., 2004; Turek et al., 2005). From a clinical perspective it is important to understand, which of the numerous tissue clocks contributes to metabolic homeostasis, in order to develop potent anti-obesogenic drugs, e.g. for night shift workers. While appetite regulation is mostly centrally controlled recent animal studies indicate an essential role of peripheral tissue clocks in the control of energy metabolism. For instance, local circadian oscillators in liver and pancreas where shown to regulate glucose utilization, whereas cardiomyocyte clocks are involved in cardiac repolarization (Jeyaraj et al., 2012; Lamia et al., 2008; Marcheva et al., 2010).

White adipose tissues (WATs) play a prominent role in metabolic homeostasis by storing large amounts of lipids in the form of triglycerides (TGs) that can serve as fuel substrate for other organs. During extended periods of energy shortage (e.g. during fasting, but also during the daily rest phase) the release of lipids from WAT mediated by the hydrolysis of TG (lipolysis) to free fatty acids (FFAs) and glycerol becomes an important energy source. The timing of FFA release from adipose stores has 
to be tightly controlled as excess of circulating lipids may lead to lipotoxicity and promote cardiovascular disorders (Unger et al., 2010). Previous reports showed that adipose tissues exhibit rhythmic clock gene expression in mice and man (Ando et al., 2005; Otway et al., 2011; Zvonic et al., 2006). Cell-based and animal studies suggest that clock genes are positive regulators of adipogenesis (Grimaldi et al., 2010; Shimba et al., 2005). It remains unclear, however, how circadian disruption may lead to increased adipose tissue deposition and obesity, as observed in human shift workers and in various clock gene mutant mice.

In this study we analyze the role of WAT clocks in lipid utilization in mice. We show that selfsustained local clocks are critical regulators of rhythmic FFA release from WAT stores, thus revealing a novel and peripherally regulated mechanism by which circadian disruption may impinge on energy homeostasis.

\section{Circadian Clock ${ }^{\Delta 19}$ mutants show increased adiposity and blunted FFA and glycerol rhythms in blood}

To gain more insights into the circadian regulation of lipid homeostasis we compared diurnal profiles of various lipid parameters in serum between circadian clock deficient Clock ${ }^{\Delta 19}$ and wild-type mice. After entrainment to $12 \mathrm{hrs}$ light: $12 \mathrm{hrs}$ dark conditions (LD) animals were released into constant darkness (DD) and sacrificed at four different time points over the course of the day. Consistent with a previous report (Turek et al., 2005), Clock $^{\Delta 19}$ mice had significantly higher cholesterol levels in the blood, albeit no significant rhythmicity in daily concentrations was observed in either genotype (Figure 1A). In contrast, serum triglyceride levels were mildly rhythmic, but indistinguishable between genotypes (Figure 1B). Interestingly, FFA serum levels showed a robust circadian pattern in wild-type animals. This rhythm was abolished and overall levels were decreased in $\mathrm{Clock}^{119}$ mutants (Figure 1C), suggesting an involvement of the circadian clock machinery in the regulation of fatty acid release from TG stores. In line with this hypothesis, serum glycerol concentrations showed robust variations in wild-type serum, while in $\mathrm{Clock}^{\Delta 19}$ mutants levels were non-rhythmic and overall low (Figure 1D). Similar FFA and glycerol changes were also observed under LD conditions (Figure S1A) and in another clock deficient mouse model, Bmal1-- (Figure S1B). Surprisingly, while blood TG and FFA levels were unaltered or low, Clock ${ }^{\Delta 19}$ mutants at the same time exhibited higher overall body weight with increased adiposity (Figure 1E and 1F) (Turek et al., 2005). Histological analysis of WAT revealed increased WAT lipid accumulation and adipocyte hypertrophy in Clock $^{\Delta 19}$ mutant mice (Figure 1G), suggesting that the Clock mutation may promote lipid accumulation or inhibit lipid mobilization in WAT. 
A

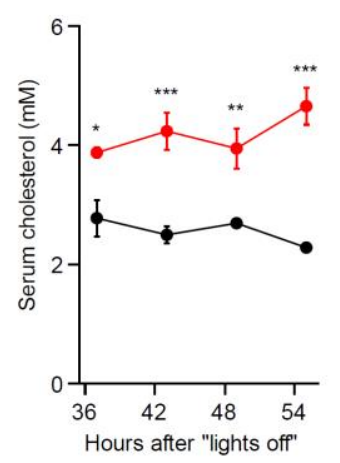

E

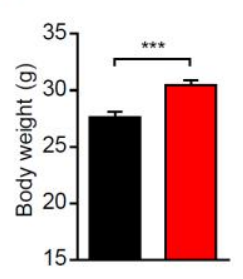

B

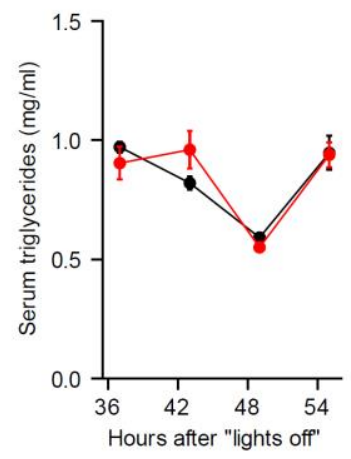

G
C

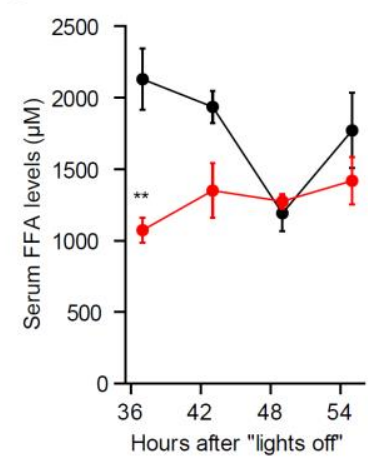

D

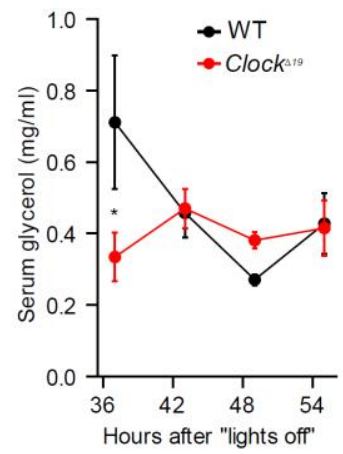

$\mathrm{F}$

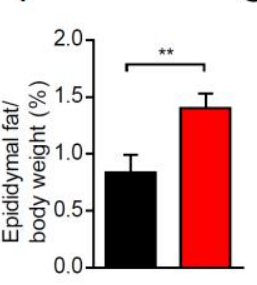

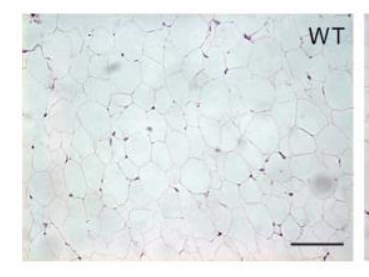
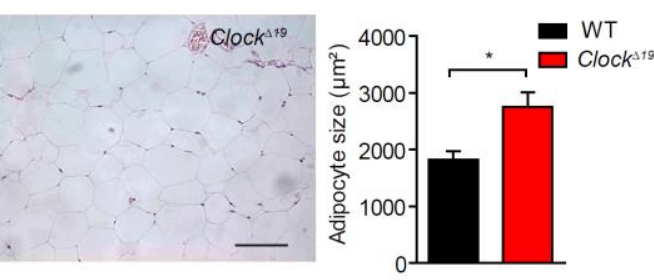

Figure 1. Serum lipid changes and adiposity in $\mathrm{Clock}^{\Delta 19}$ mice.

(A-D) Diurnal profiles of cholesterol (A), triglycerides (B), FFAs (C) and glycerol (D) in the serum of wild-type (black line) and $\mathrm{Clock}^{\Delta 19}$ (red line) mice in DD ( $\mathrm{n}=3$ per time point). Time points indicate hours spent in constant darkness (DD) after last "lights off" $\left({ }^{*} p<0.05,{ }^{* *} p<0.01,{ }^{* * *} p<0.001\right.$ by 2 -way ANOVA with Bonferroni posttest).

( $E$ and $F$ ) Body weight ( $E$ ) and adiposity (epididymal fat to body weight ratio) (F) in wild-type (black bars) and Clock $^{\Delta 19}$ (red bars) mice fed a standard diet for 10 weeks $\left(n=12,{ }^{*} p<0.05,{ }^{* *} p<0.01,{ }^{* * *} p<0.001\right.$ by unpaired $t$-test).Data for (E) and (F) were provided by Dr. Judit Meyer-Kovac.

(G) Representative sections and adipocyte size of epididymal WAT after 10 weeks of standard diet (scale bar is $100 \mu \mathrm{m} ; 15$ cells per section and 2 section per mouse and 3 mice per genotype were measured), $* p<0.05$, by unpaired $t$-test). All data are shown as means \pm SEM.

\section{Circadian regulation of genes involved in WAT lipid metabolism}

Circadian clocks regulate local cellular physiology via transcriptional programs involving large numbers of tissue-specific clock-controlled genes (Panda et al., 2002). To test if molecular clocks are involved in regulating WAT physiology we analyzed circadian variations in mRNAs of genes involved in WAT lipid metabolism. Genes were selected using the Gene Ontology database (http://www.geneontology.org) and expression data assembled on BioGPS (http://biogps.org) and 
compared to published literature (Figure S2A). mRNA levels of each gene were assessed by

A

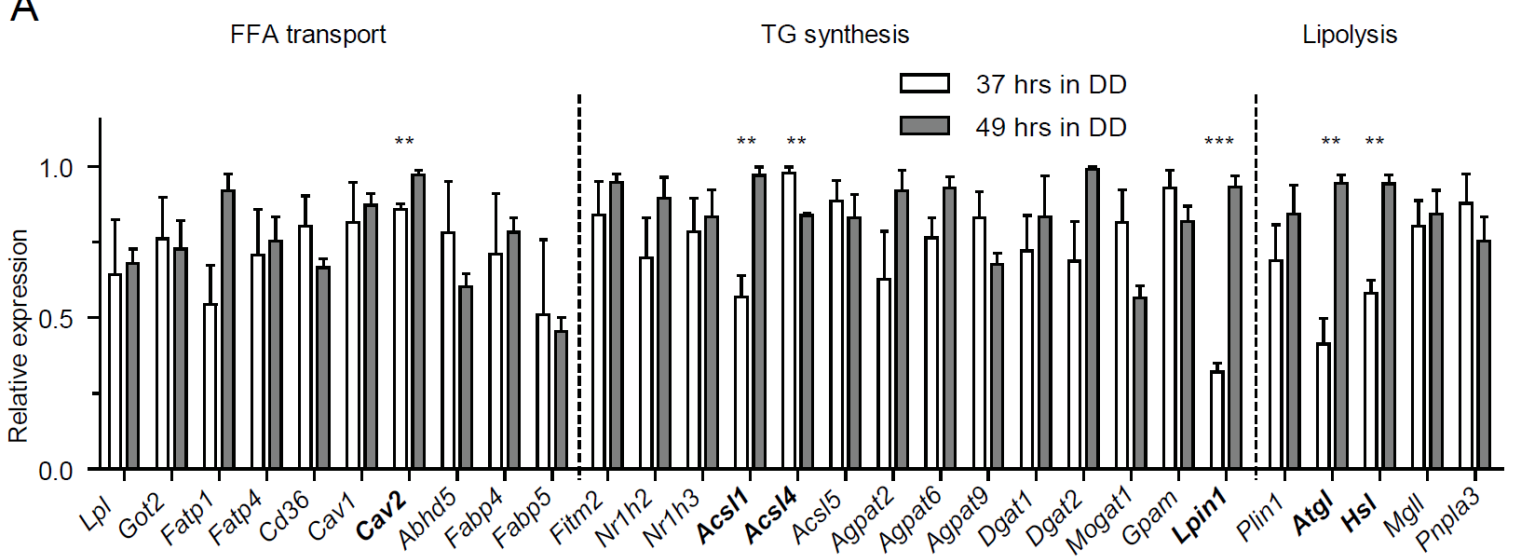

B

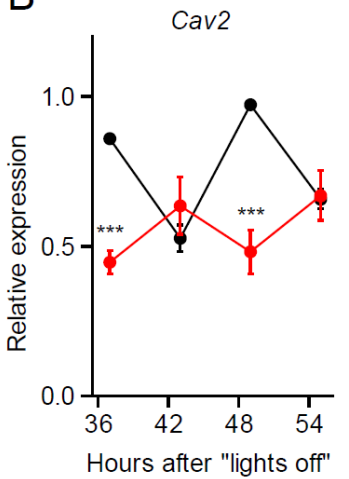

D

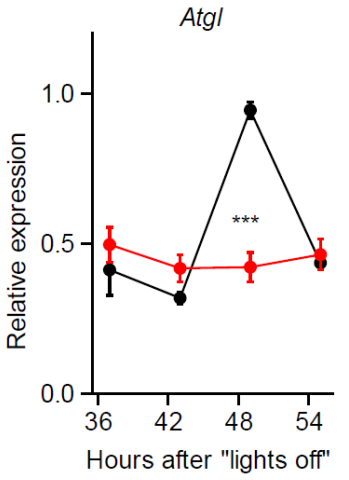

C
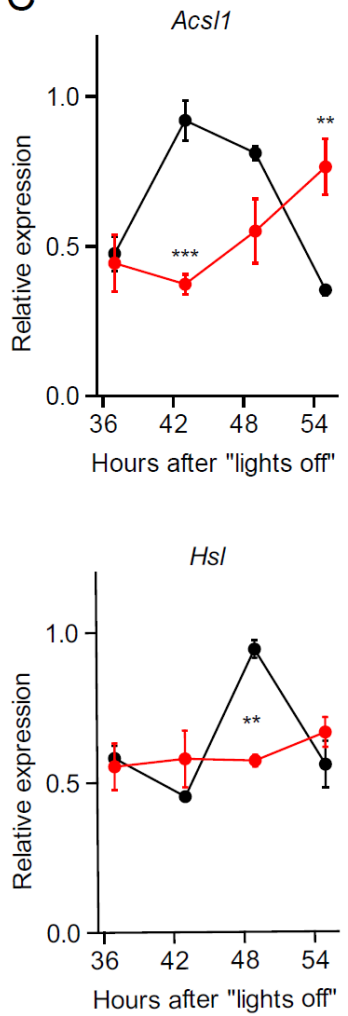
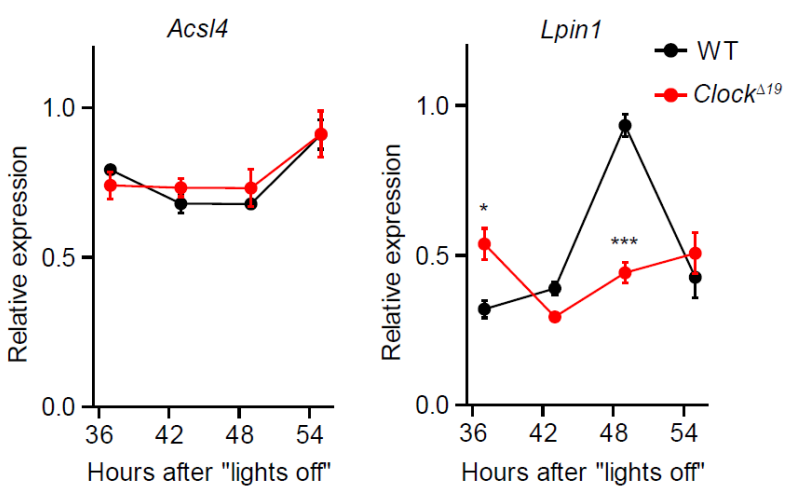

$\mathrm{E}$

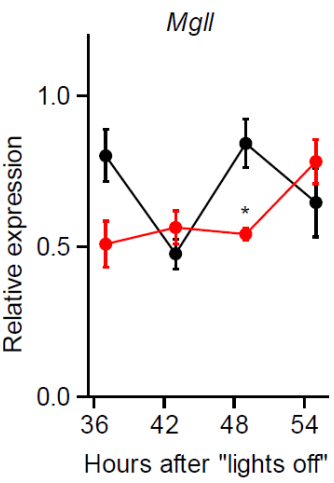

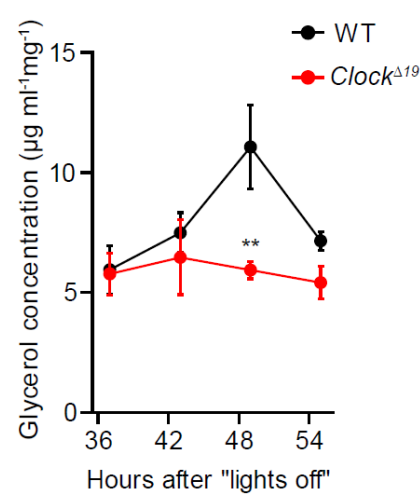

Figure 2. Circadian control of TG metabolism in WAT.

(A) qPCR screen for clock-regulated genes involved in adipocyte TG metabolism from wild-type WAT samples isolated at two opposite circadian time points, 37 hours and 49 hours in DD ( $n=3$ per time point; ${ }^{*} p<0.05,{ }^{* *} p$ $<0.01$ by unpaired t-test).

(B-D) Circadian expression profiles of genes involved in FFA transport (B), TG synthesis (C) and lipolysis (D) in WAT samples from wild-type (black line) and $\operatorname{Clock}^{\Delta 19}$ (red line) mice in DD ( $n=3$ per time point; ${ }^{*} p<0.05, * * p<$ $0.01, * * * p<0.001$ by 2 -way ANOVA with Bonferroni post-test).

(E) Profiles of glycerol excretion from wild-type (black line) and $\operatorname{Clock}^{\Delta 19}$ (red line) epididymal WAT fat pad explants harvested in DD ( $\mathrm{n}=8-9$ per time point; ${ }^{*} \mathrm{p}<0.05,{ }^{* *} \mathrm{p}<0.01,{ }^{* * *} \mathrm{p}<0.001$ by 2-way ANOVA with Bonferroni post-test). All data are shown as means \pm SEM. 
quantitative real-time PCR (qPCR) from wild-type epididymal white adipose tissue sampled at two time points, at the beginning of the rest phase (37 hours in DD) and in the early subjective night (49 hours in DD). For those genes which showed significant differences in mRNA levels between both time points we determined full circadian transcriptional profiles in WAT of Clock $^{\Delta 19}$ and wild-type mice. Of the genes associated with FFA transport and TG biosynthesis (i.e. the conversion of FFAs for storage in adipose lipid droplets) Calveolin2 (Cav2), the acyl-CoA synthetases Acs/1, Acs/4, and the phosphatidate phosphatase Lpin1 showed significant differences in expression between 37 and 49 hrs in DD (Figure 2A). In Clock $^{\Delta 19}$ mutants FFA transport/TG biosynthesis associated circadian mRNA profiles were either unaffected $(A c s / 4, L p /)$ or dampened and overall down-regulated when compared to wild-type controls (Figure $2 \mathrm{~B}$ and $2 \mathrm{C}$; Figure S2B). Such reduced expression of genes involved in lipid uptake and storage did not correlate well with the increased adiposity observed in Clock $^{\Delta 19}$ mice (Figure 1). In contrast, of the genes involved in lipolysis (i.e. the hydrolytic mobilization of lipid droplet TGs) the mRNAs of two rate-limiting enzymes, adipose triglyceride lipase (Atgl or Pnpla2) and hormone-sensitive lipase (Hs/ or Lipe), exhibited circadian variations in wild-type animals (Figure $2 \mathrm{~A}$ ). Atgl and $\mathrm{Hs} /$ transcription rates (together with that of $\mathrm{Mg} / \mathrm{l}$ ) were significantly reduced in Clock $^{\Delta 19}$ mutants (Figure 2D), strongly correlating with the reduced and arrhythmic FFA/glycerol serum levels observed in these mice. To test TG breakdown from WAT stores more directly we performed lipolysis assays on epididymal fat pad explants at four different time points in DD and LD. In both conditions, glycerol excretion showed a rhythmic pattern in wild-type mice, whereas dampened rhythmicity and a general down-regulation of lipolysis rates were observed in explants of $\mathrm{Clock}^{\Delta 19}$ mice (Figure 2E; Figure S2C). Analogously, Bmal1 ${ }^{-1}$ mice also showed lower expression levels of Atgl and Hsl (Figure S2D) and Bmal1-deficient fat pads displayed lower lipolysis rates (Figure S2E and S2F).

\section{Adipose peripheral clocks drive rhythmic expression of Atgl and HsI}

To further specify the circadian regulation of gene expression in WAT we analyzed mRNA profiles of Bmal1, Per2 and the clock output gene Dbp in DD. Expression of Bmal1, Per2 and Dbp genes exhibited strong circadian variations in wild-type animals, which were significantly down-regulated and arrhythmic (Per2, Dbp) or dampened (Bmal1) in Clock $^{\Delta 19}$ mutant mice (Figure 3A). To characterize the sustainability of molecular circadian rhythms in WAT and compare clock function between different fat depots we cultured epididymal, perirenal, peritoneal, subcutaneous white as well as intrascapular brown adipose tissue (BAT) fat explants from PER2::LUCIFERASE circadian reporter mice. All cultures showed sustained bioluminescence rhythms in the circadian range for several days (Figure 3B; Figure S3A) (Yoo et al., 2004). The phases of luminescent oscillations were very comparable between different depots and to those reported from other peripheral tissue 
explants (Pezuk et al., 2010; Yoo et al., 2004) (Figure S3B). Periodogram analysis revealed

A

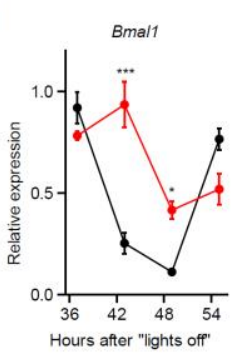

C
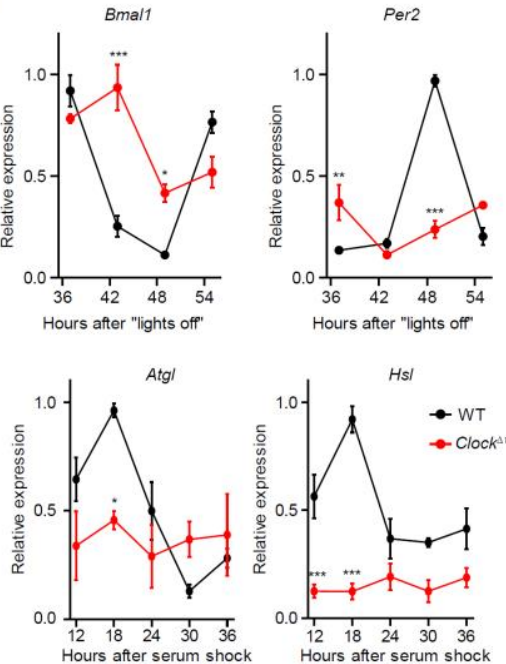

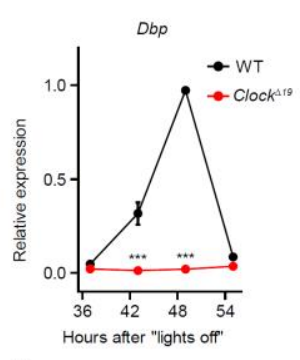

D

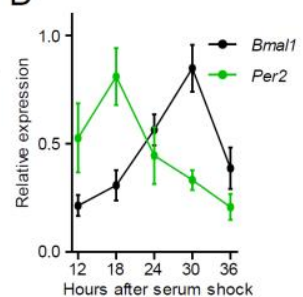

B
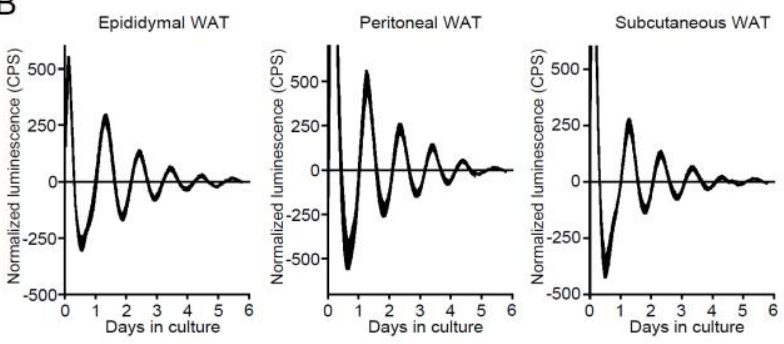

E
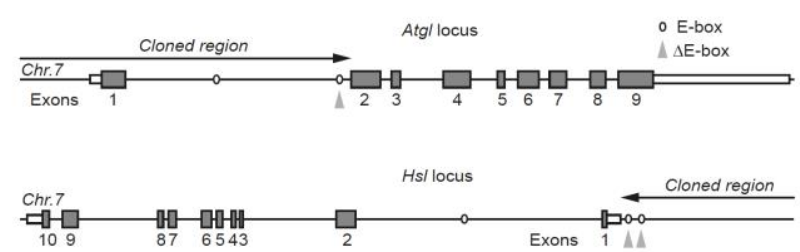

G
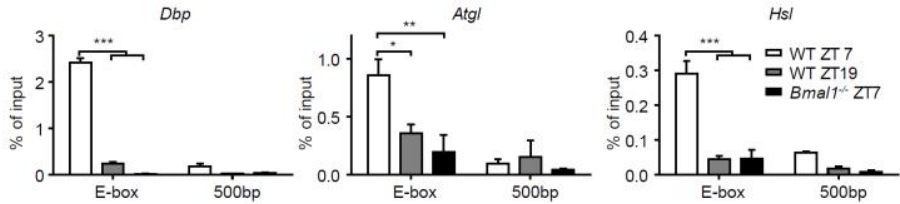

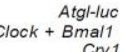
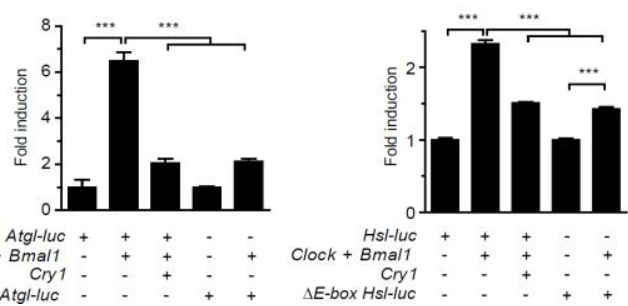

Figure 3. WAT clocks regulate expression of Atgl and Hsl via CLOCK and BMAL1.

(A) Expression profiles of Bmal1, Per2 and Dbp mRNAs in WAT of wild-type (black line) and Clock ${ }^{\Delta 19}$ (red line) mice in DD ( $n=3$ per time point; $* p<0.05, * * p<0.01, * * * p<0.001$ by 2 -way ANOVA with Bonferroni posttest).

(B) Representative baseline-subtracted luminescence recordings from epididymal, peritoneal and subcutaneous WAT explants of PER2::LUCIFERASE circadian reporter mice.

(C) Rhythmic expression of Atgl and Hs/ transcripts in wild-type (black line), but not Clock ${ }^{\Delta 19}$ (red line) cultured fat pad explants ( $n=3$ per time point; $* p<0.05, * * p<0.01, * * * p<0.001$ by 2 -way ANOVA with Bonferroni post-test).

(D) Anti-phasic expression of Per2 (green line) and Bmal1 (black line) mRNAs in cultured wild-type fat pad explants ( $n=3$ per time point; $* p<0.05, * * p<0.01, * * * p<0.001$ by 2 -way ANOVA with Bonferroni post-test).

(E) Maps of the 5' regions of the genomic loci of murine Atgl (upper panel) and Hsl (lower panel) on chromosome 7. Putative E-box enhancers are indicated by ovals. Black arrows depict the genomic sequences cloned for promoter studies. Mutated E-boxes are indicated by gray arrowheads.

(F) Luciferase reporter assays in HEK293 cells for wild-type and mutated Atgl (left) and Hsl (right) promoters in response to co-transfection with Clock/Bmal1 and Cry1 $(n=3 ; * * * p<0.001$ by 1-way ANOVA with Bonferroni post-test). 
(G) Time-dependent BMAL1 binding to Dbp, Atgl and $\mathrm{Hsl}$ E-boxes and 500 base pairs downstream regions as identified by ChIP ( $\mathrm{n}=3$ per time point; ${ }^{*} \mathrm{p}<0.05,{ }^{* *} \mathrm{p}<0.01,{ }^{* * *} \mathrm{p}<0.001$ by 2 -way ANOVA with Bonferroni post-test). All data are shown as means \pm SEM.

endogenous periodicities of around $25 \mathrm{hrs}$; statistically significant differences in period where only observed between epididymal WAT and BAT (25.8 \pm 0.2 vs. $24.7 \pm 0.3$ hrs) (Figure S3C). Moreover, rhythm dampening was comparable among all adipose tissues tested, indicating that the clock gene machinery is similarly regulated between different adipose depots (Figure S3D). To test if $\mathrm{Atgl}$ and $\mathrm{Hsl}$ transcription is clock driven at the tissue level we kept epididymal fat explants in culture for 36 hours. We observed rhythmic Atgl and Hs/ mRNAs expression in wild-type fat pads ( $p=0.0004$ and $p=0.006$, respectively). Similar to what was observed in vivo, transcript rhythms were abolished and overall levels were reduced in explant cultures from Clock $^{\Delta 19}$ mice ( $p>0.8$ for both genes) (Figure 3C). Remarkably, the expression rhythms of the two lipolytic genes were in phase coherence with the Ebox-regulated Per2 and anti-phasic to Bmal1 (compare Figures $3 \mathrm{C}$ and 3D). Taken together, these data suggest a direct regulation of $\mathrm{Atgl}$ and $\mathrm{Hs}$ l expression by CLOCK and BMAL1.

\section{BMAL1 and CLOCK regulate expression of Atgl and Hsl via E-boxes}

We identified two canonical E-box sequences (CACGTG) in the first intron of Atgl (chr7:148642099148642104, 148642860-148642866) and in the upstream region of Hsl (chr7:26181156-26181161, 26181424-26181430). Genomic DNA fragments containing these cis-regulatory elements were cloned into pGL3/4 vector and used for luciferase-based transactivation assays in HEK293A cells (Figure 3E). Co-transfection with Clock and Bmal1 increased the activity of both Atgl and Hsl promoters by 6.5 and 2.3 fold, respectively (Figure 3F). This activation was inhibited in both cases by co-transfection with Cry1, a negative E-box regulator. Moreover, mutation of the E-box proximal to the second exon of the Atgl gene (chr7:148642860-148642866) abolished transcriptional activation by CLOCK/BMAL1 (Figure 3F, left). Similar results were obtained upon mutation of both upstream Eboxes in the $\mathrm{Hsl}$ promoter (Figure 3F, right). To confirm direct BMAL1 binding of these promoters in vivo, we performed chromatin immunoprecipitation (ChIP) on epididymal adipose tissue sampled at two different time points. ChIP analysis revealed time-of-day dependent occupancy of BMAL1 on $\mathrm{Dbp}, \mathrm{Atgl}$ and $\mathrm{Hsl} \mathrm{E}$-boxes, but not on $500 \mathrm{bp}$ downstream regions in wild-type mice. In WAT of Bmal1-deficient animals BMAL1 binding signal was markedly reduced (Figure 3G). Finally, we transfected Atgl-luc and Hsl-luc constructs into NIH-3T3 cells and recorded bioluminescence for 48 hrs after serum shock synchronization. We observed a rhythmic bioluminescence signal for Atgl-luc and Hsl-luc anti-phasic to Bmal1-luc (Figure S3E and S3F), comparable to what has been reported for 
other E-box controlled genes such as Per2 (Meng et al., 2008). Together, these results strongly suggest that circadian Atgl and $\mathrm{Hs}$ I transcription is directly regulated by CLOCK/BMAL1 via E-box activation at the adipose tissue level.

A
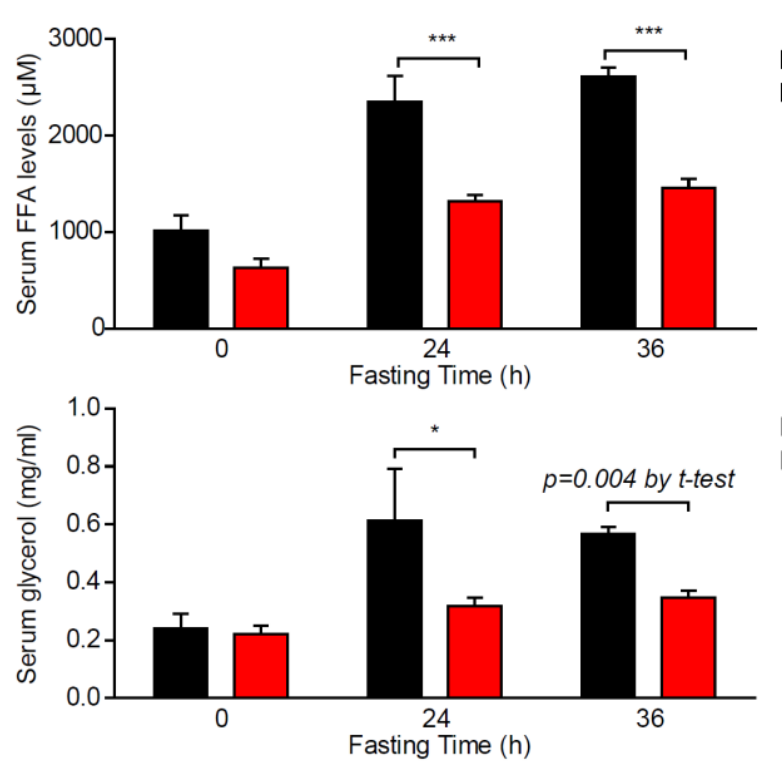

B

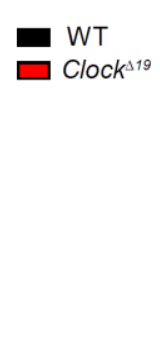

C

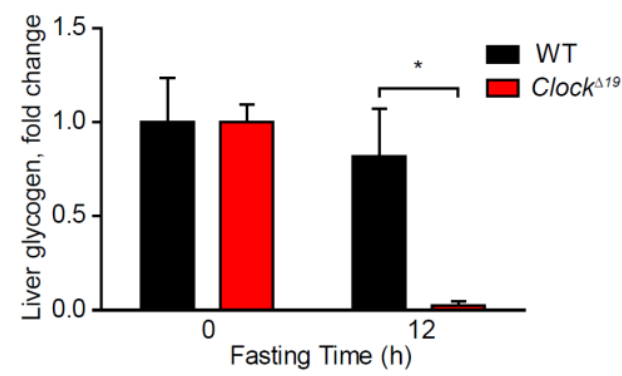

WT

$\square$ Clock $^{\Delta 19}$

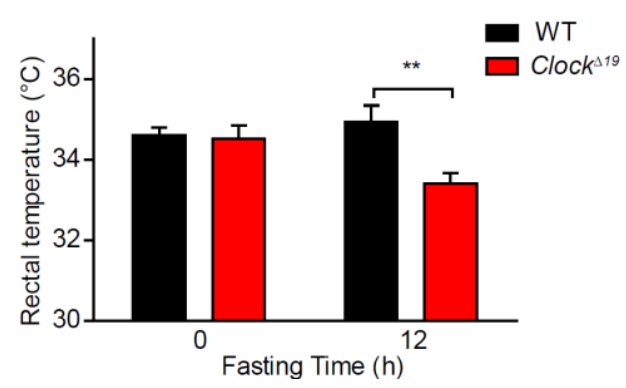

Figure 4. Aberrant fasting response in $\mathrm{Clock}^{\Delta 19}$ mice.

(A) Serum FFA and glycerol levels after 24 and 36 hrs of food deprivation ( $n=3-4$ per time point; ${ }^{*} p<0.05, * * * p$ $<0.001$ by 2-way ANOVA with Bonferroni post-test).

(B) Normalized changes in liver glycogen content of wild-type (black bars) and Clock $^{\Delta 19}$ (red bars) mice during 12 hrs of fasting ( $n=3-4 ; * p<0.05$ by 2-way ANOVA with Bonferroni post-test).

(C) Rectal temperature in wild-type (black bars) and $\operatorname{Clock}^{\Delta 19}$ (red bars) animals during 12 hrs of fasting ( $\mathrm{n}=6-12$; ${ }^{*} p<0.05, * * p<0.01$, by 2 -way ANOVA with Bonferroni post-test). All data are shown as means \pm SEM.

\section{Defective lipolysis and fasting intolerance in $\mathrm{Clock}^{\Delta 19}$ mutant mice}

Lipolysis becomes an important energy source during the fasting (i.e. rest) phase of the day. In line with this reduced FFA mobilization provokes aberrant physiological responses under fasting conditions (Wu et al., 2012). To test if fasting responses are impaired in Clock ${ }^{\Delta 19}$ mutants we fooddeprived mutant and age-matched wild-type mice starting at the end of the light phase (ZT12). Indeed, both FFA and glycerol levels were upregulated in serum in response to 24 and $36 \mathrm{hr}$ starve periods in wild-types, whereas the starve-induced lipolytic response was severely dampened in Clock $^{\Delta 19}$ animals (Figure 4A). Given that WAT FFA release - and, thus, the availability of lipids as energy source - appeared impaired we speculated that under fasting conditions mutants may rely more heavily on carbohydrate or protein utilization (Wu et al., 2012). In line with this, liver glycogen 
stores in Clock $^{\Delta 19}$ mice were depleted much faster than in wild-types under fasting conditions (Figure 4B). Moreover, after food removal mutants showed lower rectal temperature when compared to wild-type controls, implying decreased thermogenesis due to general energy shortage (Figure 4C).

Circadian physiological rhythms are generated by interplay between systemic time cues, e.g. rhythmic hormones or metabolites, and the orchestration of transcriptional programs by local tissue clocks. As a result many physiological parameters which exhibit diurnal variations are altered in mice with perturbed molecular clocks (Marcheva et al., 2010; Rudic et al., 2004). Here we report that blood FFA and glycerol concentrations show strong variations across the day. In humans it has been shown that this phenomenon is not solely a response to rhythmic food intake since FFA and glycerol are still rhythmic under constant routine conditions (Dallmann et al., 2012). In an attempt to describe the underlying mechanisms we focused our attention on circadian clocks of white adipose tissues as the main lipid stores of the body and, thus, a critical regulator of metabolic homeostasis.

We screened for diurnal rhythms in transcripts involved in adipocyte lipid metabolism. Together with a few other candidates we identified Atgl and $\mathrm{Hsl}$, which encode for two lipolysis pacemaker enzymes responsible for more than 95\% of TG hydrolysis activity (Schweiger et al., 2006). During fasting conditions, e.g. during the daily rest phase, adipose-released FFAs become an important energy source (Ahmadian et al., 2009; Duncan et al., 2007). Therefore mobilization of FFAs is critically involved in the regulation of metabolic homeostasis. In WAT expression of Atgl and Hsl showed a robust circadian rhythm in vivo that was down-regulated in $\mathrm{Clock}^{\Delta 19}$ and $\mathrm{Bmall}^{-1}$ mutants, indicating regulation by the local circadian clock machinery. Rhythmic clock gene expression has been demonstrated in many tissues in rodents and humans including various adipose depots (Ando et al., 2005; Otway et al., 2011; Zvonic et al., 2006). Using PER2::LUCIFERASE fat pad explants cultures we showed that adipose clocks show similar endogenous rhythm sustainment described for other central and peripheral oscillators. Rhythmic Atgl and Hsl expression was also sustained in fat pads explants ex-vivo. Moreover reporter gene assay and ChIP indicated that CLOCK/BMAL1 directly bind the promoter regions of $\mathrm{Atgl}$ and $\mathrm{Hsl}$. In summary, our results strongly suggest that both genes are at least in part - under local E-box-mediated transcriptional regulation by a WAT circadian clock.

In line with previous studies in rats (Benavides et al., 1998; Tsutsumi et al., 2002), we showed circadian rhythms in baseline WAT lipolysis rates and FFA levels in the blood of wild-type mice. In $\mathrm{Clock}^{\Delta 19}$ and Bmal1 ${ }^{-1}$ mutants both these parameters were flattened and down-regulated (our data and (Kennaway et al., 2007; Oishi et al., 2006)), indicating defects in lipid mobilization. It has previously been demonstrated that $\mathrm{Bmal1}^{-}$mice are heavier than wild-type littermates and exhibit 
higher fat content during the first months before they succumb to a premature ageing phenotype (Guo et al., 2012; Hemmeryckx et al., 2011; Lamia et al., 2008). Moreover, C57BL/6 Clock ${ }^{\Delta 19}$ animals are obese and are more prone to develop metabolic syndrome under high-fat diet conditions (Turek et al., 2005). Similar effects have been reported after genetic deletion of $\mathrm{Hsl}$ and Atgl. Hsl-deficient mice show hypertrophic adipocytes, reduced lipolysis rates and decreased FFA levels, though their body weight is normal (Osuga et al., 2000). Atgl conventional and adipocyte-specific mutants display the same phenotype together with increased body weight (Ahmadian et al., 2011; Haemmerle et al., 2006; Hoy et al., 2011; Huijsman et al., 2009), suggesting that low lipolysis rates lead to TG accumulation in WAT and promote obesity. Mice with defective lipolysis exhibit impaired metabolic compensation during food deprivation (Wu et al., 2012). Consistently, our data reveal that Clock ${ }^{\Delta 19}$ mutants show aberrant fasting responses. During the inactive phase wild-type mice switch their energy substrate preference to lipids which corresponds to a lower respiratory exchange ratio (RER) (Satoh et al., 2006). Hsl and Atgl-deficient animals, however, continue to primarily utilize carbohydrates during that time due to impaired FFA release into the blood (Huijsman et al., 2009). Remarkably, a similar RER phenotype was already described for $\mathrm{Clock}^{-/}$and $\mathrm{Bmal1}^{-/}$mice indicating impaired lipid metabolism (Eckel-Mahan et al., 2012; Shimba et al., 2011).

So far the mechanistic link between circadian disruption and associated metabolic defects is not clearly understood. Recent reports suggest that alterations in diurnal feeding rhythms may contribute to the development of obesity after circadian disruption (Barclay et al., 2012; Hatori et al., 2012; Turek et al., 2005). Here we show that in addition white adipose clocks directly regulate diurnal lipid homeostasis by controlling FFA/glycerol mobilization from WAT stores via transcriptional regulation of the lipolysis pacemaker enzymes Atgl and $\mathrm{Hsl}$ (Figure S4). By this adipocyte clock function may directly impinge on energy homeostasis, thus providing a potential new target for the treatment of obesity and obesity-associated disorders, e.g. in human shift workers. 


\section{Supplementary Figures}

A
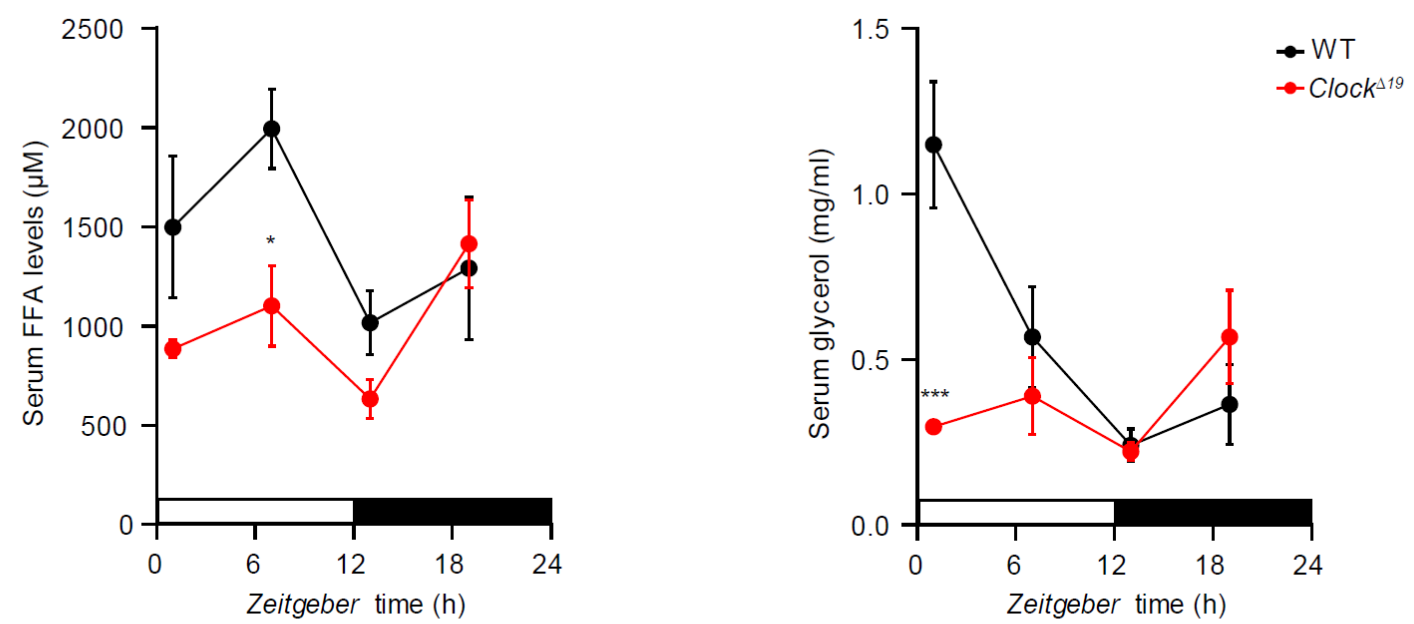

B

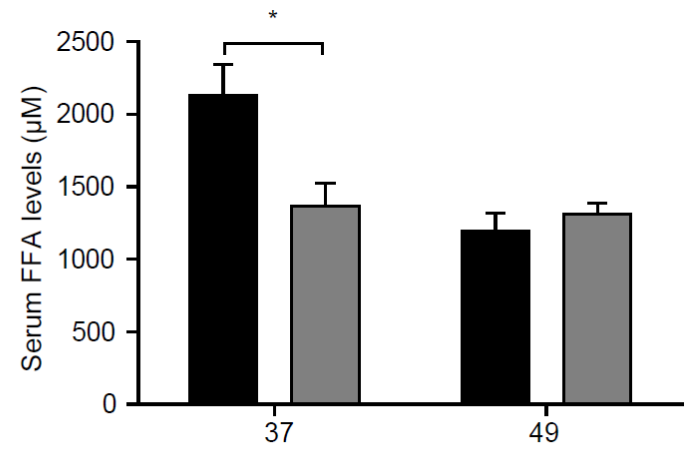

Hours after "lights off"

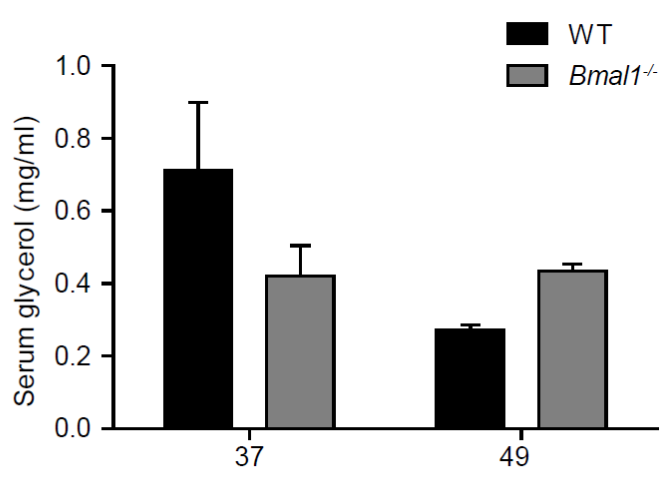

Hours after "lights off"

Figure S1. Serum FFA and glycerol levels in $\mathrm{Clock}^{\mathrm{\Delta 19}}$ and $\mathrm{Bmal1}^{\%-}$ mice.

(A) Diurnal profiles of FFAs and glycerol in the serum of wild-type (black line) and Clock ${ }^{\Delta 19}$ (red line) mice in LD ( $n=3-5$ per time point; ${ }^{*} p<0.05, * * *<0.001$ by 2 -way ANOVA with Bonferroni post-test).

(B) Time-of-day dependent variations in serum FFA and glycerol levels in wild-type (black bars) and $B m a / 1^{-1-}$ (grey bars) animals kept in DD ( $n=2-3$ per time point; * $p<0.05$ by 2 -way ANOVA with Bonferroni post-test). All data are shown as means \pm SEM. 
A
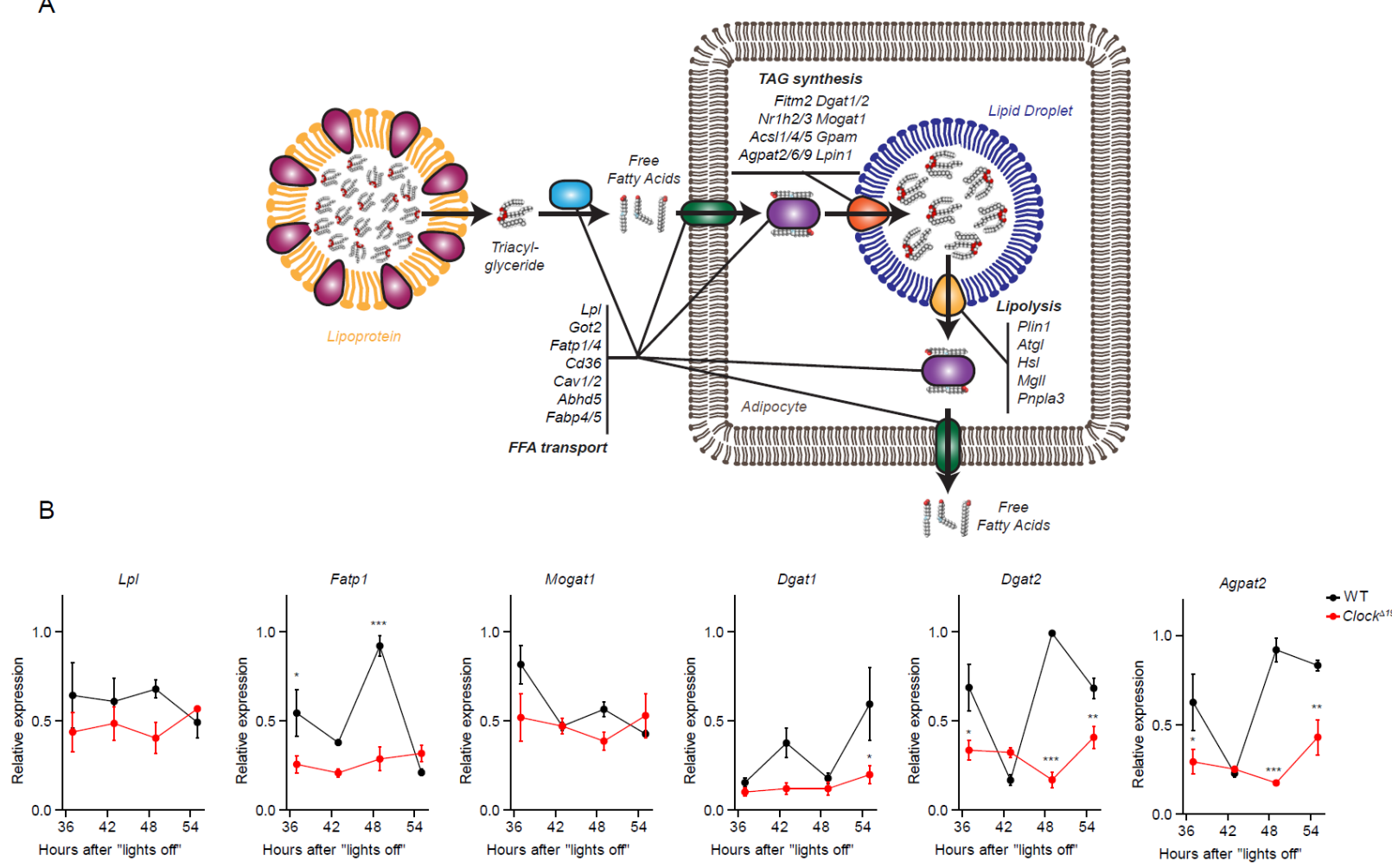

D
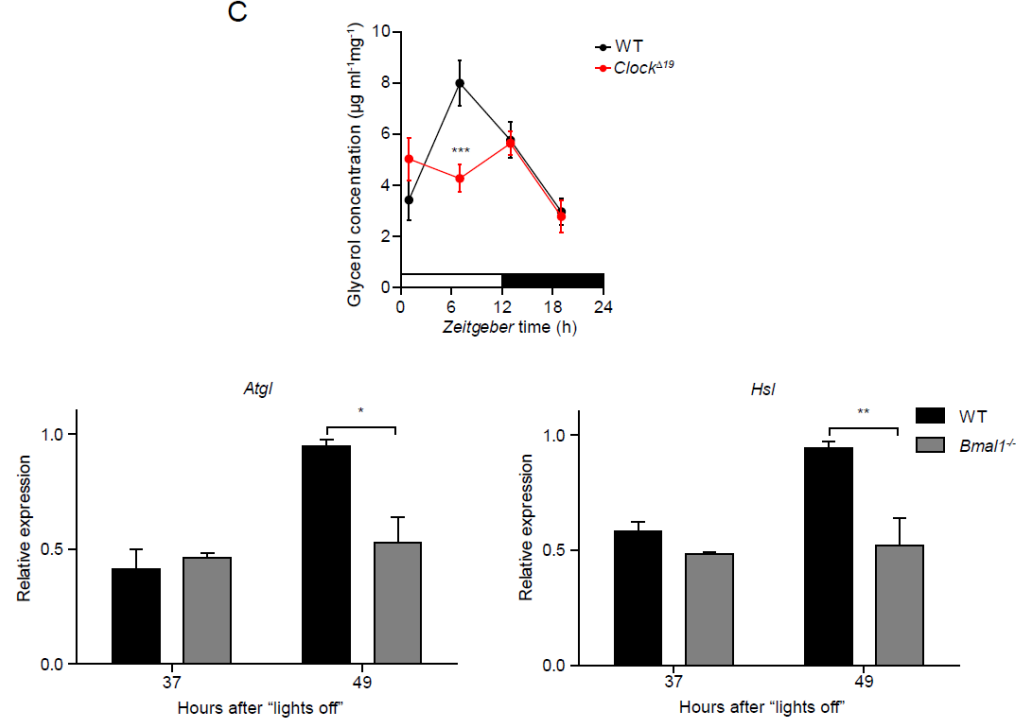

E

F
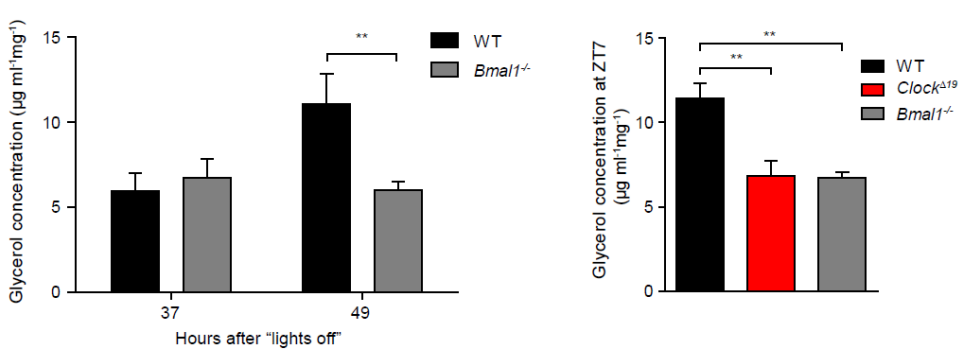

Figure S2. Circadian control of TG metabolism in WAT.

(A) Schematic overview of tested genes involved in WAT TG metabolism (assembled from Gene Ontology and BioGPS databases and literature studies). 
(B) Circadian expression profiles of genes involved in FFA transport and TG synthesis in WAT samples from wildtype (black line) and $\operatorname{Clock}^{\Delta 19}$ (red line) mice in DD ( $\mathrm{n}=3$ per time point; ${ }^{*} \mathrm{p}<0.05,{ }^{* *} \mathrm{p}<0.01,{ }^{* * *} \mathrm{p}<0.001$ by 2 way ANOVA with Bonferroni post-test).

(C) Glycerol excretion (lipolysis) profiles of wild-type (black line) and Clock ${ }^{\Delta 19}$ (red line) epididymal WAT fat pad explants harvested in LD ( $n=6-14$ per time point; *** $p<0.001$ by 2 -way ANOVA with Bonferroni post-test).

(D) Expression of Atgl and Hsl in WAT of wild-type (black bars) and Bmal1/- (grey bars) animals at 37 and $49 \mathrm{hrs}$ after lights off ( $n=2-3$ per time point; ${ }^{*} p<0.05,{ }^{*} p<0.01$, by 2 -way ANOVA with Bonferroni post-test).

(E) Changes in glycerol excretion from wild-type (black bars) and Bmal1 ${ }^{-1-}$ (grey bars) epididymal WAT fat pad explants harvested at 37 and 49 hrs after lights off ( $n=8$ per time point; ${ }^{*} p<0.05, * * p<0.01, * * * p<0.001$ by 2-way ANOVA with Bonferroni post-test).

(F) Glycerol excretion rates (lipolysis) from wild-type (black bars), Bmal1 ${ }^{-/}$(grey bars) and Clock ${ }^{\Delta 19}$ (red bars) epididymal WAT fat pad explants harvested at Zeitgeber time 7 in LD (maximum in wild-type, see also C; $n=8-9$; $* * p<0.01$ by 1 -way ANOVA with Bonferroni post-test). All data are shown as means \pm SEM. 
A

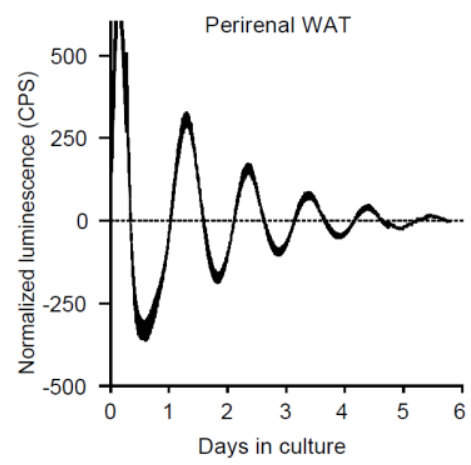

C

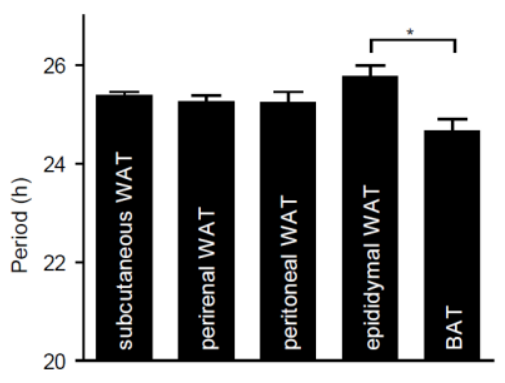

E

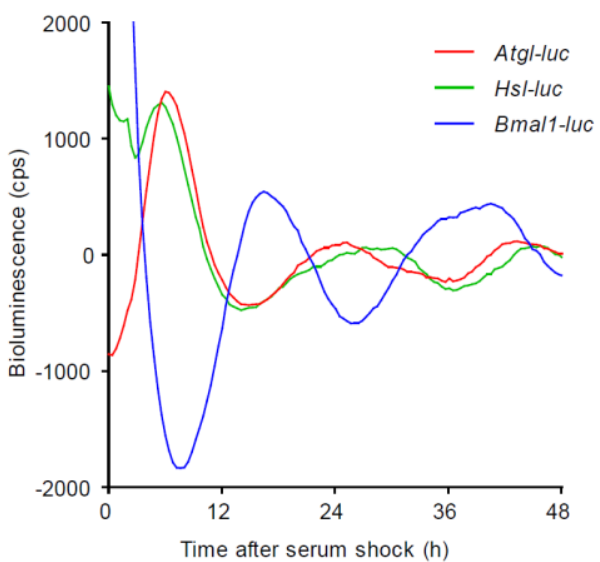

B
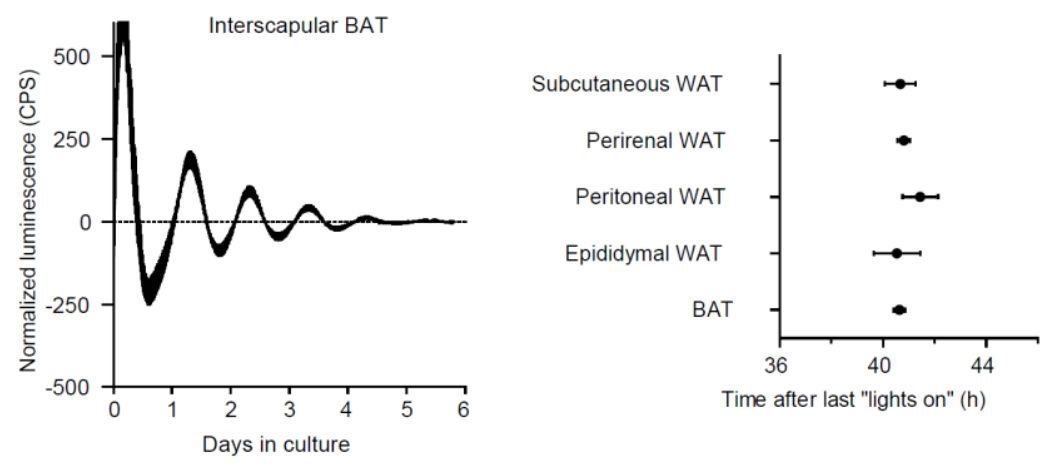

D

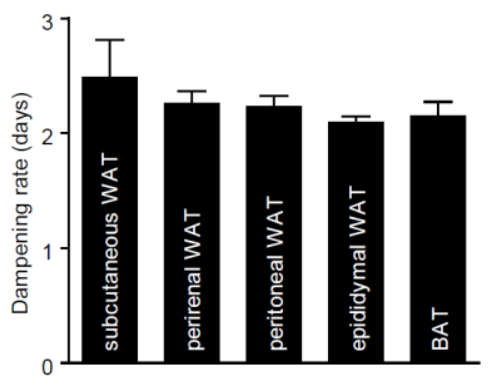

F

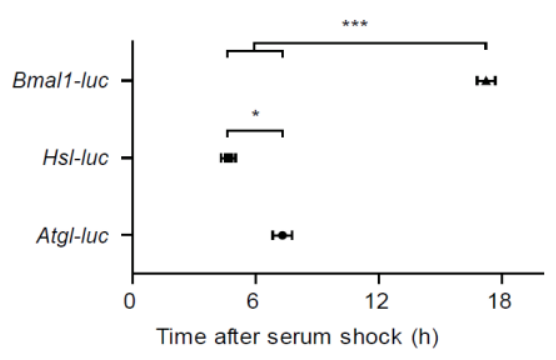

Figure S3. Sustained circadian clocks in adipose tissues.

(A) Representative PER2::LUCIFERASE recordings from perirenal WAT and interscapular BAT explants.

(B) Phase map (occurrence of the first peak) of PER2::LUCIFERASE oscillations from WAT and BAT depots relative to the last "lights on" ( $n=3-4)$.

(C and D) Period (C) and dampening rates (D) of PER2::LUCIFERASE oscillations from different fat depots ( $n=4$; $* p<0.05$, by 1-way ANOVA with Bonferroni post-test).

(E) Bioluminescence recordings of Atgl-luc, Hsl-luc and Bmal1-luc reporters from transiently transfected NIH3 T3 cells after serum shock. Raw data was 24-h baseline-subtracted and smoothened using a 1-hour running average. 
(F) Oscillation phase (occurrence of the first peak) of Atgl-luc, Hsl-luc and Bmal1-luc luminescence rhythms from transiently transfected NIH-3T3 cells after serum shock $\left(n=3-4 ;{ }^{*} p<0.05, * * * p<0.001\right.$ by 1 -way ANOVA with Bonferroni post-test). All data are shown as means \pm SEM.

\section{WT}

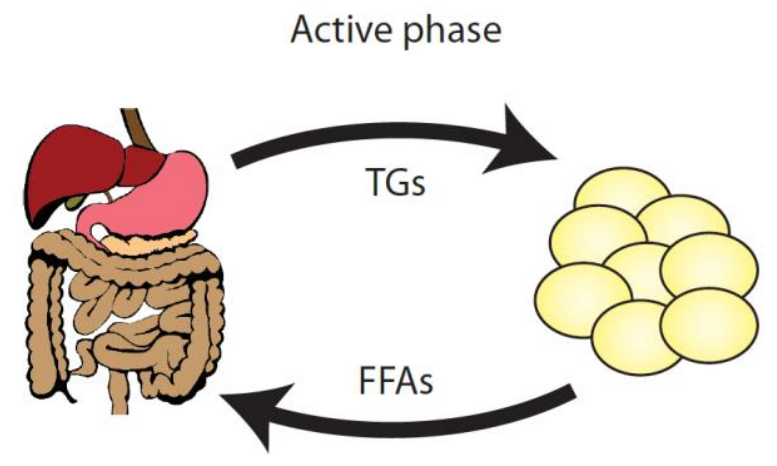

Rest phase

\section{Clock ${ }^{\Delta 19}$ and Bmal1 ${ }^{-/}$mutants}

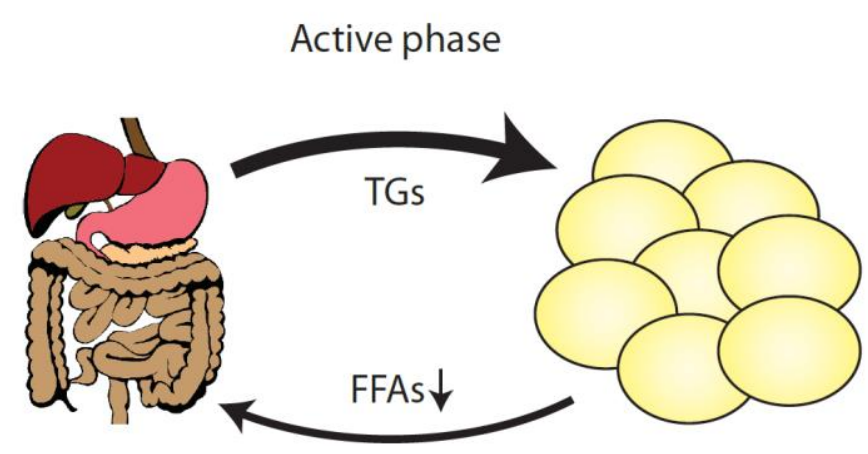

Rest phase

\section{Figure S4. Circadian regulation of lipid mobilization}

Scheme illustrating lipid metabolism during rest (light) and active phases (dark) in wild-type and $\operatorname{Clock}^{\Delta 19}$ and $B m a l 1^{-1}$ mice. During the active phase wild-type animals take up TGs with the food which is transported by lipoproteins in the blood and stored as TG in WAT lipid droplets. In the rest phase, when mice eat little, WAT lipids from WAT energy stores are mobilized by lipolysis and conversion of TG into FFAs, which are released into the blood to serve as energy substrate for the rest of the body. Impaired lipolysis in circadian mutant mice prevents efficient FFA mobilization from WAT stores, thus promoting TG accumulation and adipocyte hypertrophy, which ultimately results in increased adiposity. 


\section{Methods}

\section{Animals}

Male wild-type (C57BL/6) and congenic homozygous Clock ${ }^{\Delta 19}$ (Vitaterna et al., 1994) and Bmal1 ${ }^{-/}$ mice (Bunger et al., 2000) of 2-4 months of age were used for all experiments. All animal experiments were done after ethical assessment and licensed by the Office of Consumer Protection and Food Safety of the State of Lower Saxony and in accordance with the German Law of Animal Welfare. Mice were housed in small groups of 5 or fewer under 12 hrs light: 12 hrs dark cycle (LD) or constant darkness (DD) conditions with food and water access ad libitum.

\section{Fat pad cultures}

Wild-type or PER2::LUCIFERASE mice (Yoo et al., 2004) were LD entrained and sacrificed by cervical dislocation at Zeitgeber time (ZT) 9 (i.e. 3 hrs before "lights off"). WAT and BAT samples were isolated and washed with 1x HBSS (Hank's balanced salt solution) (PAA, Cölbe, DE). Tissues were divided into 20-30 mg pieces and cultured in colorless DMEM media (Dulbecco's modified Eagle medium)(PAA) supplemented with 10\% FBS (PAA) and $100 \mathrm{nM}$ luciferin sodium salt (Biosynth, Staad, CH). For RNA extraction fat pads were removed at 6-hr intervals and total RNA was extracted and processed as described below. Bioluminescence recordings were performed using a Lumicycle luminometer (Actimetrics, Willmette, IL). Period and damping rate were calculated using the Lumicycle Analysis software (Actimetrics).

\section{Gene expression analysis using quantitative real-time (q)PCR}

Wild-type and homozygous Clock $^{\Delta 19}$ mice were entrained to LD for at least 2 weeks, released into DD and sacrificed at 37, 43, 49, and 55 hours after "lights off" (corresponding to circadian times (CT) 1, 7, 13, and 19, respectively). For LD cohorts mice were kept under LD and sacrificed at ZT1, 7, 13, and 19. Epididymal fat was isolated and total RNA was extracted using TRIzol reagent (Life Technologies, Darmstadt, DE). cDNA synthesis was performed by reverse transcription (Life Technologies) using random hexamer primers. qPCR was performed using iQ SYBR Green Supermix on an CFX96 thermocycler (Bio-Rad, Munich, DE) according to the manufacturer's protocol. Relative gene expression was quantified using a $\Delta \Delta \mathrm{CT}$ method and Eef1 $\alpha$ as reference genes (Oster et al., 2006). Primer sequences are listed in Materials and Methods. 


\section{Cloning of Atgl and Hsl promoters and PCR mutagenesis}

A $4.3 \mathrm{~kb}$ fragment containing the murine Atgl upstream sequence and the first intron was PCRamplified from genomic DNA using Advantage 2 polymerase mix (Clontech, Mountain View, CA). PCR products were digested with HindIII (NEB, Ipswich, MA) and Xhol (NEB) and cloned into HindIII/Xholdigested pGL4 vector (Promega, Madison, WI). A $4.5 \mathrm{~kb}$ fragment of the murine $\mathrm{Hs}$ promoter was PCR-amplified as described for Atgl. PCR products were cloned into $p G L 3$ vector by In-Fusion Cloning (Clontech, Saint-Germain-en-Laye, FR). E-box mutations to GGATCC were performed using a PCR mutagenesis kit (Agilent, Santa Clara, CA). Primer sequences were:

\begin{tabular}{|l|l|}
\hline $\begin{array}{l}\text { Atgl promoter } \\
\text { and } 1^{\text {st }} \text { intron } \\
\text { cloning }\end{array}$ & $\begin{array}{l}\text { Fw Pr. 5'-CTCGGCGGCCAAGCTTTCAAACCCCAGGATCTTCAACTA-3' } \\
\text { Rev Pr. 5'-TCTTGATATCCTCGAGCAGCATTCCCAGCTTAGTAACCA-3' }\end{array}$ \\
\hline $\begin{array}{l}\text { Mutation of the } \\
\text { E-box in the } \\
\text { Atgl } 1^{\text {st }} \text { intron }\end{array}$ & $\begin{array}{l}\text { Fw Pr. 5'-CCGTCTTCCCATGCTAGGCGGATCCCCATACCATGTTAGGCAC-3' } \\
\text { Rev Pr.5'-GTGCCTAACATGGTATGGGGATCCGCCTAGCATGGGAAGACGG-3' }\end{array}$ \\
\hline $\begin{array}{l}\text { Hsl promoter } \\
\text { cloning }\end{array}$ & $\begin{array}{l}\text { Fw Pr. 5'-GCAGGCATGCAAGCTTCTTTGCGCTGCCCTTATAGT -3' } \\
\text { Rev Pr. 5'- CCGGAATGCCAAGCTTGCCCTCACAGCAGGAATAGT -3' }\end{array}$ \\
\hline $\begin{array}{l}\text { Mutation of the } \\
\text { first E-box in } \\
\text { the } H \text { sl } \\
\text { promoter }\end{array}$ & $\begin{array}{l}\text { Fw Pr. 5'-AAAAAAAAAACAGGGACGACGGATCCAGGGGGCGGAGGAAAAGGC -3' } \\
\text { Rev Pr. 5'-GCCTTTTCCTCCGCCCCCTGGATCCGTCGTCCCTGTTTTTTTTTT-3' }\end{array}$ \\
\hline $\begin{array}{l}\text { Mutation of the } \\
\text { second E-box in } \\
\text { the } \mathrm{Hsl} \\
\text { promoter }\end{array}$ & $\begin{array}{l}\text { Fw Pr. 5'- CCCGCCTTTTCCGGGGGGATCCGGCTCCCTCGACTTA -3' } \\
\text { Rev Pr. 5'- TAAGTCGAGGGAGCCGGATCCCCCCGGAAAAGGCGGG -3' }\end{array}$ \\
\hline
\end{tabular}

\section{Luciferase promoter assays}

HEK293A and NIH-3T3 cells were maintained in DMEM (PAA) supplemented with 10\% FBS (PAA) and antibiotics. One day prior to transfection, cells were plated onto 24-well plates at a density of $10^{5}$ cells per well. HEK cells were co-transfected using PEI (Sigma-Aldrich, Hamburg, DE) and $50 \mathrm{ng}$ of $p G L 3 / 4$ reporter plasmid in the presence of $200 \mathrm{ng}$ of Bmal1, Clock, or Cry1 constructs. Empty pcDNA3.1 vector was used to make up the total amount of DNA to $0.7 \mu \mathrm{g}$ per well. $10 \mathrm{ng}$ of a $p R L-$ CMV Renilla luciferase reporter vector (Promega) was added to each reaction as internal control. Two days later, cells were harvested and assayed using Dual-Luciferase Reporter Assay System (Promega) on a TriStar LB941 luminometer (Berthold Technologies, Wildbach, DE). NIH-3T3 cells were transfected using X-fect (Clontech) with $1 \mu \mathrm{g} p G L 3-H s l, p G L 4-A t g l$ or Bmal1-luc (Brown et al., 2005) reporter plasmids. Two days later cells were synchronized by $50 \%$ serum shock for 2 hrs. For 
luminescence recordings cells were kept in colorless DMEM, 10 \% FBS, 100 nM luciferin sodium salt (Life Technologies).

\section{Chromatin immunoprecipitation (ChIP)}

Epididymal fat pads from wild-type and $\mathrm{Bmal1}^{-1}$ mice were isolated at ZT7 and ZT19, homogenized and immediately cross-linked in $1 \%$ formaldehyde. Chromatin was sonicated to obtain an average DNA length of approximately $400 \mathrm{bp}$ (15 s on, $20 \mathrm{sec}$ off cycles for $22 \mathrm{~min}$ on high power) using a Bioruptor (Diagenode Inc.). Samples were incubated overnight at $4{ }^{\circ} \mathrm{C}$ with antiBMAL1 antibody (N20, Santa Cruz Biotechnology, Inc.). After clearing, samples were incubated with A/G agarose beads (Thermo Scientific) for $1 \mathrm{hr}$ at $4{ }^{\circ} \mathrm{C}$ followed by intensive washings. Afterwards samples were boiled for $10 \mathrm{~min}$ in $10 \%$ Chelex (Bio-Rad, Munich, DE) with proteinase $\mathrm{K}(150 \mu \mathrm{g} / \mathrm{mL})$. After centrifugation the DNA-containing supernatant was collected for $\mathrm{qPCR}$ as described above. All values were normalized to input signal levels. Primer sequences were:

\begin{tabular}{|l|l|}
\hline$D b p$ E-box & $\begin{array}{l}\text { Fw Pr. 5'-TGGGACGCCTGGGTACAC-3' } \\
\text { Rev Pr. 5'-GGGAATGTGCAGCACTGGTT-3' }\end{array}$ \\
\hline Dbp 500bp & $\begin{array}{l}\text { Fw Pr. 5'-CGTGGAGGTGCTTAATGACCTTT-3' } \\
\text { Rev Pr.5'-CATGGCCTGGAATGCTTGA-3' }\end{array}$ \\
\hline Atg/ E-box & $\begin{array}{l}\text { Fw Pr. 5'-GGTGATGGTTGAAGTAGGTCAGA-3' } \\
\text { Rev Pr. 5'-TATTTCCCAACTGCCTGTCC-3' }\end{array}$ \\
\hline Atg/ 500bp & $\begin{array}{l}\text { Fw Pr. 5'-TTCAGACGGAGAGAACGTCA-3' } \\
\text { Rev Pr. 5'-GCAGTGCCTACCTGGATGAG-3' }\end{array}$ \\
\hline Hs/ E-box & $\begin{array}{l}\text { Fw Pr. 5'-AGCCTAGGACCCTGTCTGG-3' } \\
\text { Rev Pr. 5'-TCACGTGGTCGTCCCTGTT-3' }\end{array}$ \\
\hline Hs/ 500bp & $\begin{array}{l}\text { Fw Pr. 5'-AACTTGATCGCTGGAATTGG-3' } \\
\text { Rev Pr. 5'-GGCTCCATCAATTCTTTCCA-3' }\end{array}$ \\
\hline
\end{tabular}

\section{Lipolysis assays}

Epididymal fat pads from wild-type, homozygous $\mathrm{Clock}^{\Delta 19}$ and $\mathrm{Bmal1}^{-1-}$ mice were dissected at the indicated time points, cut into $10-25 \mathrm{mg}$ samples and incubated at $37{ }^{\circ} \mathrm{C}$ in DMEM, $10 \% \mathrm{FBS}$ with antibiotics. Glycerol release was measured from media aliquots using Free Glycerol Reagent (SigmaAldrich) and normalized to fat pad dry weight. 


\section{Blood metabolite measurements}

Trunk blood was collected at CT/ZT 1, 7, 13, and 19 and was allowed to clot. Serum was isolated by centrifugation at 2,000 $\mathrm{xg}$ for $20 \mathrm{~min}$ at $4^{\circ} \mathrm{C}$. Serum FFAs, triglyceride/glycerol and cholesterol levels were determined using NEFA kit (Zen-Bio, Research Triangle Park, NC), serum triglyceride determination kit (Sigma-Aldrich), and cholesterol assay kit (Cayman Chemical Company) according to the manufacturers' protocols.

\section{Adipocyte size determination}

Freshly isolated epididymal WAT samples from age-matched wild-type and Clock ${ }^{\Delta 19}$ animals were fixed with $4 \%$ paraformaldehyde (PFA) in 1x phosphate buffered saline (PBS) overnight. Samples were dehydrated with descending alcohol concentrations and embedded in paraffin. Sections were cut at $6 \mu \mathrm{m}$ and stained with hematoxylin-eosin. Adipocyte size was determined with Image J software (NIH, Bethesda, MA).

\section{Statistical Analyses}

Statistics were performed using Prism 5 software (GraphPad Software). P-values $<0.05$ were considered significant. Analysis of circadian gene expression was performed with CircWave software.

\section{Acknowledgements}

AS is supported by the Max Planck Society. HO is an Emmy Noether Fellow of the DFG and a Lichtenberg Fellow of the Volkswagen Foundation. The authors thank Dr. Ana Martinez Hernandez for critical reading of the manuscript and Dr. Moritz Rossner and Dr. Steve A. Brown for providing DNA constructs. 


\section{References}

Ahmadian, M., Abbott, M.J., Tang, T., Hudak, C.S., Kim, Y., Bruss, M., Hellerstein, M.K., Lee, H.Y., Samuel, V.T., Shulman, G.I., et al. (2011). Desnutrin/ATGL is regulated by AMPK and is required for a brown adipose phenotype. Cell Metab 13, 739-748.

Ahmadian, M., Duncan, R.E., and Sul, H.S. (2009). The skinny on fat: lipolysis and fatty acid utilization in adipocytes. Trends Endocrinol Metab 20, 424-428.

Ando, H., Yanagihara, H., Hayashi, Y., Obi, Y., Tsuruoka, S., Takamura, T., Kaneko, S., and Fujimura, A. (2005). Rhythmic messenger ribonucleic acid expression of clock genes and adipocytokines in mouse visceral adipose tissue. Endocrinology 146, 5631-5636.

Barclay, J.L., Husse, J., Bode, B., Naujokat, N., Meyer-Kovac, J., Schmid, S.M., Lehnert, H., and Oster, H. (2012). Circadian desynchrony promotes metabolic disruption in a mouse model of shiftwork. PLoS One 7, e37150.

Benavides, A., Siches, M., and Llobera, M. (1998). Circadian rhythms of lipoprotein lipase and hepatic lipase activities in intermediate metabolism of adult rat. Am J Physiol 275, R811-817.

Bray, M.S., and Young, M.E. (2007). Circadian rhythms in the development of obesity: potential role for the circadian clock within the adipocyte. Obes Rev 8, 169-181.

Brown, S.A., Ripperger, J., Kadener, S., Fleury-Olela, F., Vilbois, F., Rosbash, M., and Schibler, U. (2005). PERIOD1-associated proteins modulate the negative limb of the mammalian circadian oscillator. Science 308, 693-696.

Bunger, M.K., Wilsbacher, L.D., Moran, S.M., Clendenin, C., Radcliffe, L.A., Hogenesch, J.B., Simon, M.C., Takahashi, J.S., and Bradfield, C.A. (2000). Mop3 is an essential component of the master circadian pacemaker in mammals. Cell 103, 1009-1017.

Dallmann, R., Viola, A.U., Tarokh, L., Cajochen, C., and Brown, S.A. (2012). The human circadian metabolome. Proc Natl Acad Sci U S A 109, 2625-2629.

Duncan, R.E., Ahmadian, M., Jaworski, K., Sarkadi-Nagy, E., and Sul, H.S. (2007). Regulation of lipolysis in adipocytes. Annu Rev Nutr 27, 79-101.

Eckel-Mahan, K.L., Patel, V.R., Mohney, R.P., Vignola, K.S., Baldi, P., and Sassone-Corsi, P. (2012). Coordination of the transcriptome and metabolome by the circadian clock. Proc Natl Acad Sci U S A $109,5541-5546$.

Grimaldi, B., Bellet, M.M., Katada, S., Astarita, G., Hirayama, J., Amin, R.H., Granneman, J.G., Piomelli, D., Leff, T., and Sassone-Corsi, P. (2010). PER2 controls lipid metabolism by direct regulation of PPARgamma. Cell Metab 12, 509-520. 
Guo, B., Chatterjee, S., Li, L., Kim, J.M., Lee, J., Yechoor, V.K., Minze, L.J., Hsueh, W., and Ma, K. (2012). The clock gene, brain and muscle Arnt-like 1, regulates adipogenesis via Wnt signaling pathway. FASEB J.

Haemmerle, G., Lass, A., Zimmermann, R., Gorkiewicz, G., Meyer, C., Rozman, J., Heldmaier, G., Maier, R., Theussl, C., Eder, S., et al. (2006). Defective lipolysis and altered energy metabolism in mice lacking adipose triglyceride lipase. Science 312, 734-737.

Harmer, S.L., Panda, S., and Kay, S.A. (2001). Molecular bases of circadian rhythms. Annu Rev Cell Dev Biol 17, 215-253.

Hatori, M., Vollmers, C., Zarrinpar, A., Ditacchio, L., Bushong, E.A., Gill, S., Leblanc, M., Chaix, A., Joens, M., Fitzpatrick, J.A., et al. (2012). Time-Restricted Feeding without Reducing Caloric Intake Prevents Metabolic Diseases in Mice Fed a High-Fat Diet. Cell Metab 15, 848-860.

Hemmeryckx, B., Himmelreich, U., Hoylaerts, M.F., and Lijnen, H.R. (2011). Impact of clock gene Bmal1 deficiency on nutritionally induced obesity in mice. Obesity (Silver Spring) 19, 659-661. Hoy, A.J., Bruce, C.R., Turpin, S.M., Morris, A.J., Febbraio, M.A., and Watt, M.J. (2011). Adipose triglyceride lipase-null mice are resistant to high-fat diet-induced insulin resistance despite reduced energy expenditure and ectopic lipid accumulation. Endocrinology 152, 48-58.

Huijsman, E., van de Par, C., Economou, C., van der Poel, C., Lynch, G.S., Schoiswohl, G., Haemmerle, G., Zechner, R., and Watt, M.J. (2009). Adipose triacylglycerol lipase deletion alters whole body energy metabolism and impairs exercise performance in mice. Am J Physiol Endocrinol Metab 297, E505-513.

Jeyaraj, D., Haldar, S.M., Wan, X., McCauley, M.D., Ripperger, J.A., Hu, K., Lu, Y., Eapen, B.L., Sharma, N., Ficker, E., et al. (2012). Circadian rhythms govern cardiac repolarization and arrhythmogenesis. Nature 483, 96-99.

Kennaway, D.J., Owens, J.A., Voultsios, A., Boden, M.J., and Varcoe, T.J. (2007). Metabolic homeostasis in mice with disrupted Clock gene expression in peripheral tissues. Am J Physiol Regul Integr Comp Physiol 293, R1528-1537.

Lamia, K.A., Storch, K.F., and Weitz, C.J. (2008). Physiological significance of a peripheral tissue circadian clock. Proc Natl Acad Sci U S A 105, 15172-15177.

Marcheva, B., Ramsey, K.M., Buhr, E.D., Kobayashi, Y., Su, H., Ko, C.H., Ivanova, G., Omura, C., Mo, S., Vitaterna, M.H., et al. (2010). Disruption of the clock components CLOCK and BMAL1 leads to hypoinsulinaemia and diabetes. Nature 466, 627-631.

Meng, Q.J., McMaster, A., Beesley, S., Lu, W.Q., Gibbs, J., Parks, D., Collins, J., Farrow, S., Donn, R., Ray, D., et al. (2008). Ligand modulation of REV-ERBalpha function resets the peripheral circadian clock in a phasic manner. J Cell Sci 121, 3629-3635. 
Oishi, K., Atsumi, G., Sugiyama, S., Kodomari, I., Kasamatsu, M., Machida, K., and Ishida, N. (2006). Disrupted fat absorption attenuates obesity induced by a high-fat diet in Clock mutant mice. FEBS Lett $580,127-130$.

Oster, H., Damerow, S., Kiessling, S., Jakubcakova, V., Abraham, D., Tian, J., Hoffmann, M.W., and Eichele, G. (2006). The circadian rhythm of glucocorticoids is regulated by a gating mechanism residing in the adrenal cortical clock. Cell Metab 4, 163-173.

Osuga, J., Ishibashi, S., Oka, T., Yagyu, H., Tozawa, R., Fujimoto, A., Shionoiri, F., Yahagi, N., Kraemer, F.B., Tsutsumi, O., et al. (2000). Targeted disruption of hormone-sensitive lipase results in male sterility and adipocyte hypertrophy, but not in obesity. Proc Natl Acad Sci U S A 97, 787-792.

Otway, D.T., Mantele, S., Bretschneider, S., Wright, J., Trayhurn, P., Skene, D.J., Robertson, M.D., and Johnston, J.D. (2011). Rhythmic diurnal gene expression in human adipose tissue from individuals who are lean, overweight, and type 2 diabetic. Diabetes 60, 1577-1581.

Panda, S., Antoch, M.P., Miller, B.H., Su, A.I., Schook, A.B., Straume, M., Schultz, P.G., Kay, S.A., Takahashi, J.S., and Hogenesch, J.B. (2002). Coordinated transcription of key pathways in the mouse by the circadian clock. Cell 109, 307-320.

Pezuk, P., Mohawk, J.A., Yoshikawa, T., Sellix, M.T., and Menaker, M. (2010). Circadian organization is governed by extra-SCN pacemakers. J Biol Rhythms 25, 432-441.

Rudic, R.D., McNamara, P., Curtis, A.M., Boston, R.C., Panda, S., Hogenesch, J.B., and Fitzgerald, G.A. (2004). BMAL1 and CLOCK, two essential components of the circadian clock, are involved in glucose homeostasis. PLoS Biol 2, e377.

Satoh, Y., Kawai, H., Kudo, N., Kawashima, Y., and Mitsumoto, A. (2006). Time-restricted feeding entrains daily rhythms of energy metabolism in mice. Am J Physiol Regul Integr Comp Physiol 290, R1276-1283.

Schweiger, M., Schreiber, R., Haemmerle, G., Lass, A., Fledelius, C., Jacobsen, P., Tornqvist, H., Zechner, R., and Zimmermann, R. (2006). Adipose triglyceride lipase and hormone-sensitive lipase are the major enzymes in adipose tissue triacylglycerol catabolism. J Biol Chem 281, 40236-40241.

Shimba, S., Ishii, N., Ohta, Y., Ohno, T., Watabe, Y., Hayashi, M., Wada, T., Aoyagi, T., and Tezuka, M. (2005). Brain and muscle Arnt-like protein-1 (BMAL1), a component of the molecular clock, regulates adipogenesis. Proc Natl Acad Sci U S A 102, 12071-12076.

Shimba, S., Ogawa, T., Hitosugi, S., Ichihashi, Y., Nakadaira, Y., Kobayashi, M., Tezuka, M., Kosuge, Y., Ishige, K., Ito, Y., et al. (2011). Deficient of a clock gene, brain and muscle Arnt-like protein-1 (BMAL1), induces dyslipidemia and ectopic fat formation. PLoS One 6, e25231.

Storch, K.F., Lipan, O., Leykin, I., Viswanathan, N., Davis, F.C., Wong, W.H., and Weitz, C.J. (2002). Extensive and divergent circadian gene expression in liver and heart. Nature 417, 78-83. 
Tsutsumi, K., Inoue, Y., and Kondo, Y. (2002). The relationship between lipoprotein lipase activity and respiratory quotient of rats in circadian rhythms. Biol Pharm Bull 25, 1360-1363.

Turek, F.W., Joshu, C., Kohsaka, A., Lin, E., Ivanova, G., McDearmon, E., Laposky, A., Losee-Olson, S., Easton, A., Jensen, D.R., et al. (2005). Obesity and metabolic syndrome in circadian Clock mutant mice. Science 308, 1043-1045.

Unger, R.H., Clark, G.O., Scherer, P.E., and Orci, L. (2010). Lipid homeostasis, lipotoxicity and the metabolic syndrome. Biochim Biophys Acta 1801, 209-214.

Vitaterna, M.H., King, D.P., Chang, A.M., Kornhauser, J.M., Lowrey, P.L., McDonald, J.D., Dove, W.F., Pinto, L.H., Turek, F.W., and Takahashi, J.S. (1994). Mutagenesis and mapping of a mouse gene, Clock, essential for circadian behavior. Science 264, 719-725.

Wu, J.W., Wang, S.P., Casavant, S., Moreau, A., Yang, G.S., and Mitchell, G.A. (2012). Fasting energy homeostasis in mice with adipose deficiency of desnutrin/adipose triglyceride lipase.

Endocrinology 153, 2198-2207.

Yoo, S.H., Yamazaki, S., Lowrey, P.L., Shimomura, K., Ko, C.H., Buhr, E.D., Siepka, S.M., Hong, H.K., Oh, W.J., Yoo, O.J., et al. (2004). PERIOD2::LUCIFERASE real-time reporting of circadian dynamics reveals persistent circadian oscillations in mouse peripheral tissues. Proc Natl Acad Sci U S A 101, 5339-5346.

Zvonic, S., Ptitsyn, A.A., Conrad, S.A., Scott, L.K., Floyd, Z.E., Kilroy, G., Wu, X., Goh, B.C., Mynatt, R.L., and Gimble, J.M. (2006). Characterization of peripheral circadian clocks in adipose tissues.

Diabetes 55, 962-970. 


\section{Additional Data}

Stimulated lipolysis in adipose tissue of Clock ${ }^{\Delta 19}$ mutants

In order to reveal the efficacy of hormonal lipolysis induction in adipose tissue of circadian mutants, we treated fat pads with the $\beta_{1}$ - and $\beta_{2}$-adrenoreceptor agonist isoproterenol at the maximum of wild-type basal lipolysis (Zeitgeber time 7) (Haemmerle et al., 2006). We observed a strong response for both genotypes, though glycerol excretion rates from Clock ${ }^{\Delta 19}$ fat pads were slightly reduced compared to wild-type controls. These data indicate that the posttranscriptional mechanism of lipolysis induction is preserved in $\operatorname{Clock}^{\Delta 19}$ mutants. However, due to lower expression levels of Atgl and $H s l$, hormonal induction of lipolysis cannot be fully rescued (Figure 8).

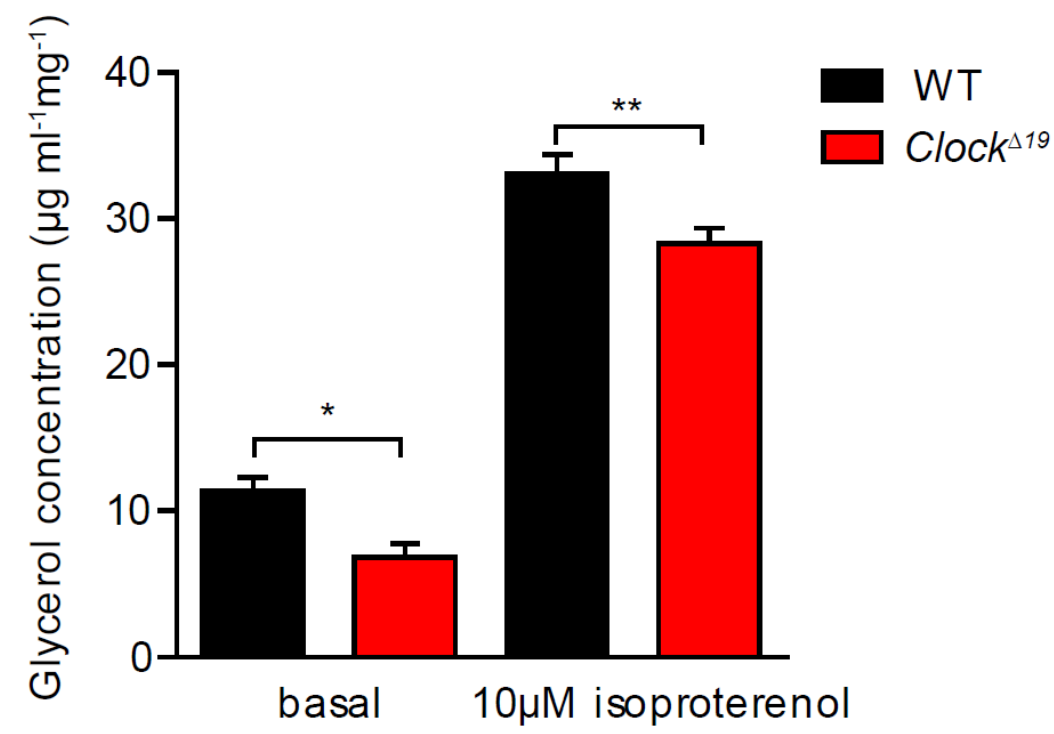

Figure 8. Isoproterenol induction of lipolysis in $\mathrm{Clock}^{\Delta 19}$ and wild-type fat pads at ZT7. Data are presented as mean \pm SEM. *, $\mathrm{P}<0.05 ; * *, \mathrm{P}<0.01$ by 2 -way ANOVA with Bonferroni post-test. 


\section{Generation of adipocyte-targeted circadian clock deficient mutant mice.}

\section{Fabp4-cre Bmal1 fl/fl animals carry a deletion of Bmal1 in adipose tissue}

To address the role of adipose tissue clocks in vivo, we generated an adipose-clock deficient mouse line crossing Fabp4-Cre mice (He et al., 2003) with Bmal1 fl/fl animals (Storch et al., 2007). By targeting the essential clock gene Bmal1 (Bunger et al., 2000) one can achieve a selective disruption of clock function in tissues expressing CRE recombinase (Lamia et al., 2008; Storch et al., 2007). After two generations of breedings we obtained the desired genotype, Fabp4-Cre Bmal1 $\mathrm{fl} / \mathrm{fl}$. We tested the efficacy and specificity of the Bmal1 deletion in different organs by preparing tissue homogenates at the maximum of Bmal1 expression (ZT18) and Western blotting with antiBMAL1 antibodies (Honma et al., 1998). As expected, we observed a deletion of BMAL1 protein from both epididymal and peritoneal fat depots (Figure 9 A and B). In contrast, we could not see any effect in other metabolically active tissues such as liver and muscle (Figure $9 \mathrm{C}$ and D). However, we unexpectedly observed a marked reduction of BMAL1 protein in the brain hinting at an ectopic expression of the CRE recombinase (Figure 9E).

A

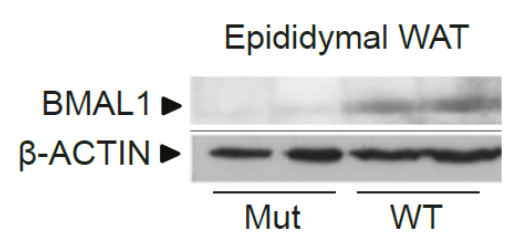

D

\section{D}

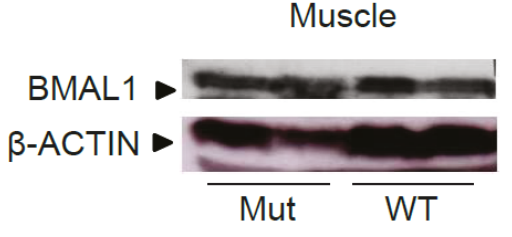

B

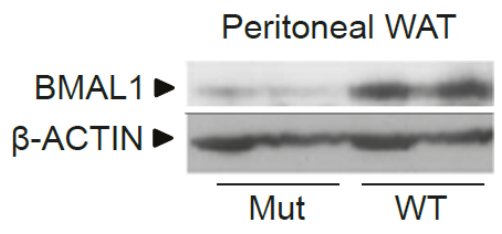

E

C
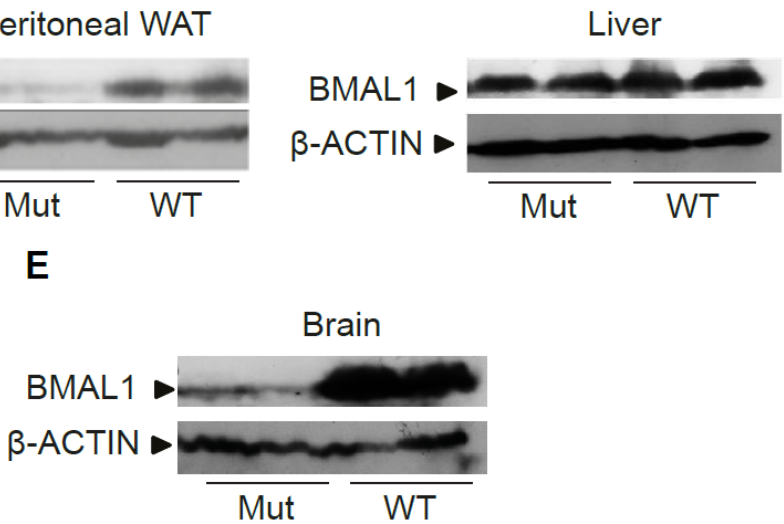

Figure 9. Western blots showing BMAL1 regulation in epididymal (A) and peritoneal (B) WATs, liver (C), muscle (D) and brain (E) in Fabp4-Cre Bmal1 fl/fl animals. Tissue protein extracts were prepared at Zeitgeber time 18 and probed with antiBMAL1 antibodies. $\beta$-ACTIN antibodies were used as loading control.

\section{Reduction of BMAL1 in the brain of Fabp4-cre Bmal1 fl/fl mice}

Next we wanted to assess the extent of BMAL1 deletion in the brain of Fabp4-Cre Bmal1 fl/fl animals. Therefore we entrained both genotypes to LD conditions and sacrificed them at ZT18. Freshly frozen brains were embedded in cryopreservation medium and subjected to cryosectioning with subsequent immunohistochemistry with antiBMAL1 antibodies. In agreement with Western blot data we revealed a general reduction of BMAL1 protein levels in brains of Fabp4-Cre Bmal1 $\mathrm{fl} / \mathrm{fl}$ mice. Concentrating our attention on the $\mathrm{SCN}$, as the brain region containing the central clock, we observed about $70 \%$ decrease of BMAL1-positive cells (Figure 10A). Similar results were obtained for 
other hypothalamic regions such as the arcuate nucleus which is important for appetite regulation (Figure 10B).

A
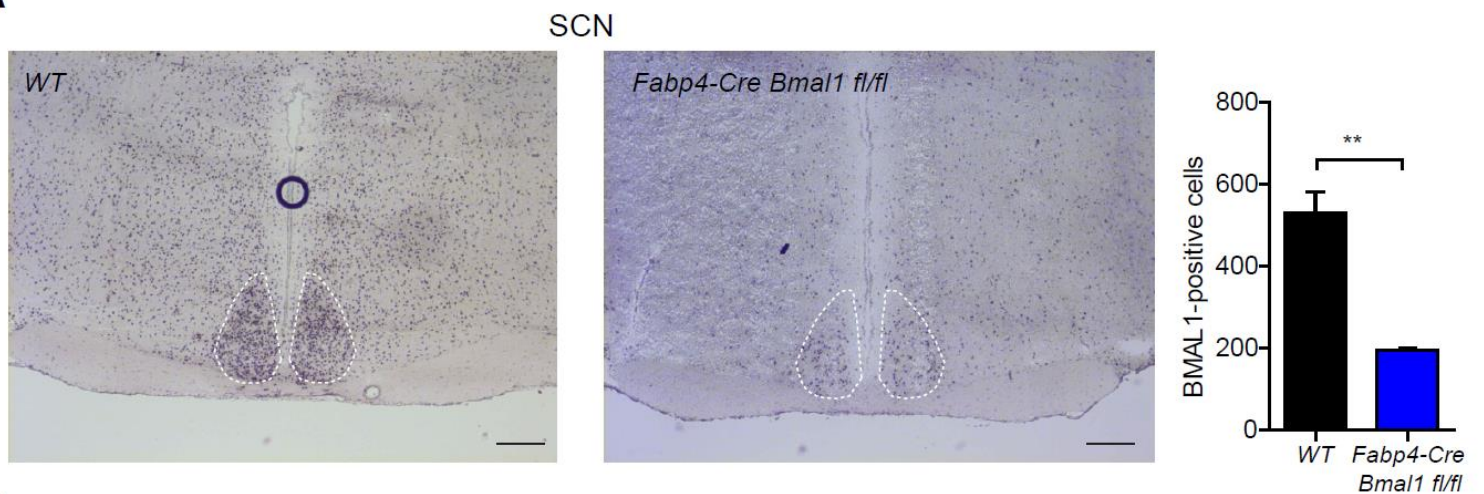

B

Arcuate nucleus
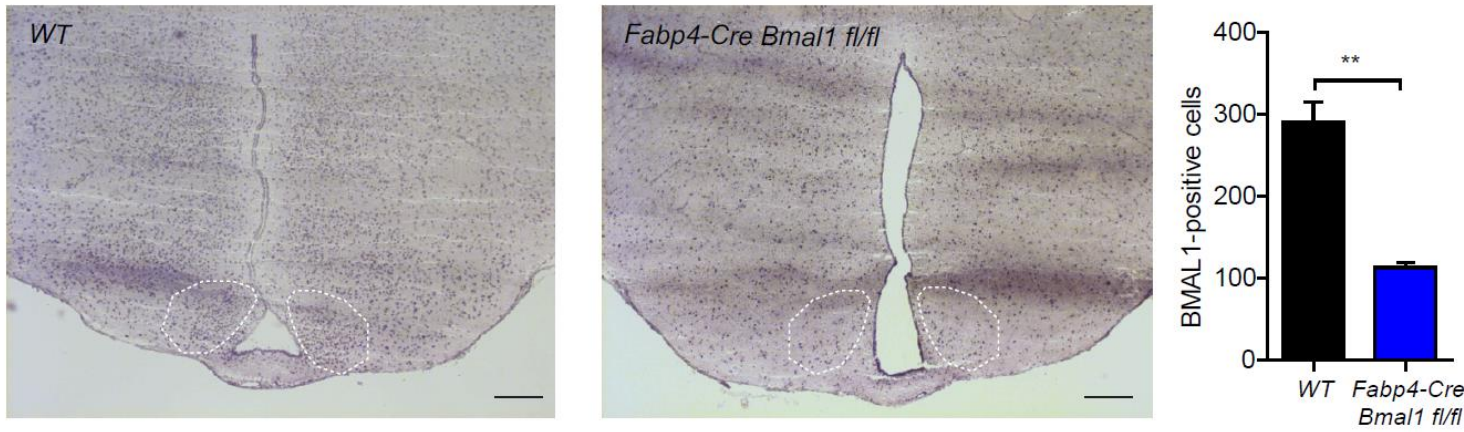

Figure 10. Quantification of BMAL1 deletion in the SCN (A) and the arcuate nucleus (B) of Fabp4-Cre Bmal1 fl/fl mice. Immunohistochemistry was performed on frozen sections with antiBMAL1 antibodies. The amount of BMAL1-positive cells was counted and compared to wild-type. Both regions are highlighted with white dashed lines. Scale bar: $0.2 \mathrm{~mm}$. Data are presented as mean \pm SEM. ( $n=3 ;{ }^{* *}, \mathrm{P}<0.01$ by unpaired $t$-test).

We tested whether such reduction of BMAL1 provokes changes in rhythmic clock gene expression in the SCN. For this purpose we entrained two cohorts of animals to LD conditions and sacrificed them each 6 hours (ZT1, ZT7, ZT13 and ZT19). Paraffin-embedded brains were sectioned and in situ hybridization with different antisense probes for clock gene transcripts was performed. Surprisingly we saw largely unchanged rhythmic mRNA levels of Bmal1 (the probe also detects the recombinant transcript, reflecting promoter activity) in the SCN of Fabp4-Cre Bmal1 fl/fl mice (Figure $11 \mathrm{~A}$, left). Moreover, two other clock genes, $D b p$ and Per2, also did not show any significant changes in their expression profiles (Figure 11A, right). To exclude the possibility of indirect effects on peripheral oscillator function in tissues that themselves do not express $\mathrm{Cre}$, we performed qPCR quantification of Bmal1 transcript levels at different time points both in liver and muscles (with primers specific for the wild-type allele). Consistent with the Western blot data (see Figure 9), we did not observe any 
changes in expression of Bmal1 (Figure 11B) indicating that clocks in non-adipose peripheral organs were intact.

A

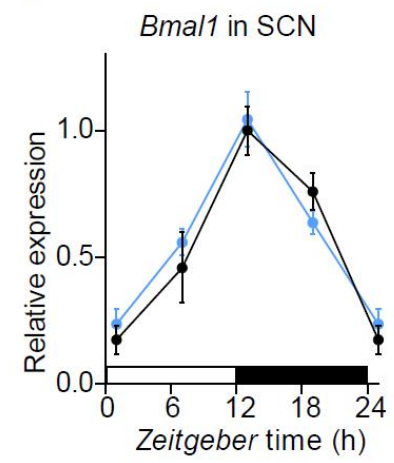

ZT1

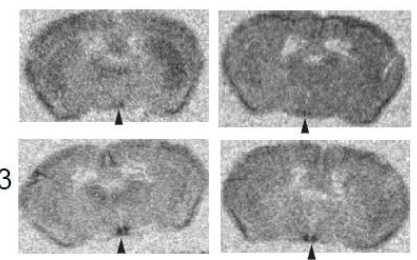

WT
Fabp4-Cre Bmal1 fl/fl

Bmal1 in liver
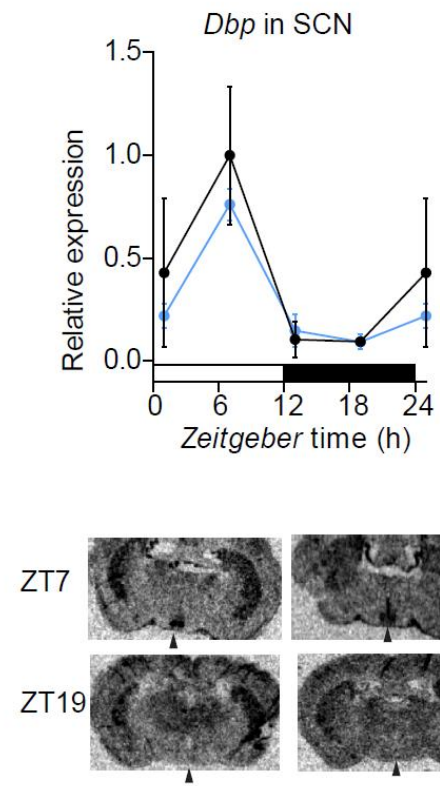

WT

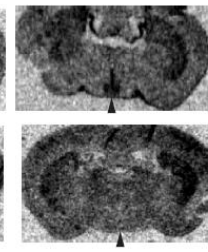

Fabp4-Cre Bmal1 fl/fl

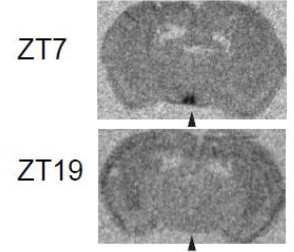

WT
Per2 in SCN

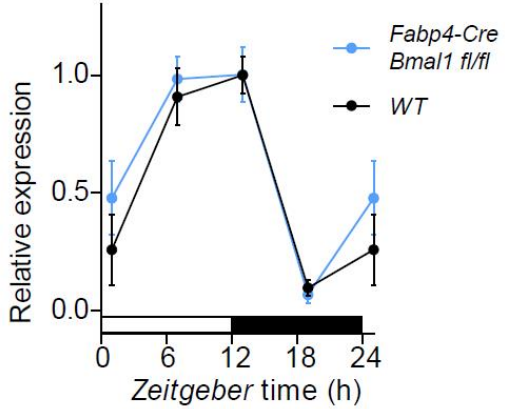

Bmal1 in muscle

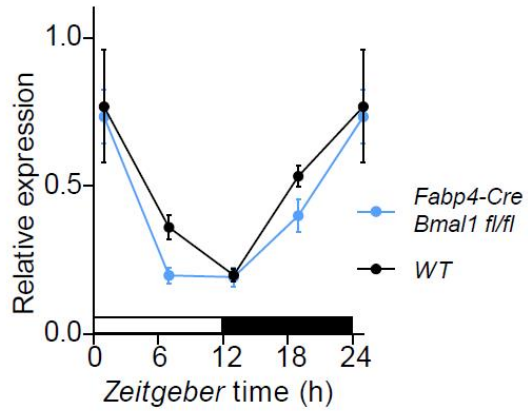

Figure 11. Clock gene expression in the SCN and peripheral tissues. (A) Bmal1, Dbp and Per2 diurnal expression profiles in the SCN region of wild-type and Fabp4-Cre Bmal1 $\mathrm{fl} / \mathrm{fl}$ mice as quantified by radioactive in situ hybridization. Representative sections are shown in the lower panel. Arrows indicate the SCN location (n=3-6 per time point). (B) Expression of Bmal1 in liver and muscle of mutant and wild-type mice quantified by qPCR ( $n=3$ per time point). Data are presented as mean \pm SEM.

\section{Behavioral phenotypes of Fabp4-Cre Bmal1 fl/fl mice}

BMAL1 protein reduction in the brain may lead to detrimental changes in circadian behavior of Fabp4-Cre Bmal1 fl/fl mice. Thus, we analyzed circadian locomotor activity with running-wheel cages under different lighting conditions. Under a 12-hrs light: 12-hrs dark regime (LD) Fabp4-Cre Bmal1 fl/fl mutants exhibited normal entrainment with activity bouts occurring mainly during the dark phase like in the wild-type situation (Figure $12 \mathrm{~A}$ and B). After one week in LD mice were transferred to constant darkness (DD) to investigate their intrinsic periodicities. In this situation, mutant animals 
showed a shorter period when compared to wild-type controls $(23.22 \pm 0.09$ hrs vs. $23.59 \pm 0.05$ hrs respectively) (Figure 12C). Moreover 70\% of individuals showed gradual loss of rhythmicity when kept in DD (data not shown). Nevertheless mutants showed no obvious defects in entraining their behavior to LD conditions.

A

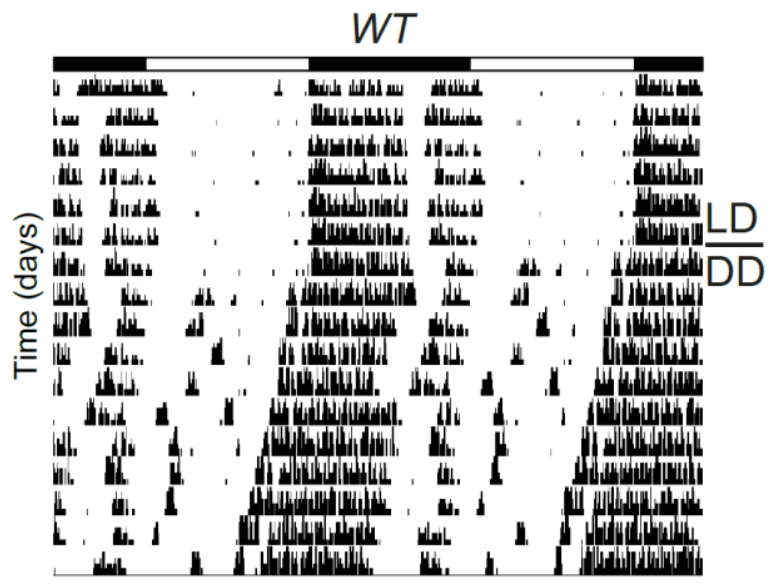

B

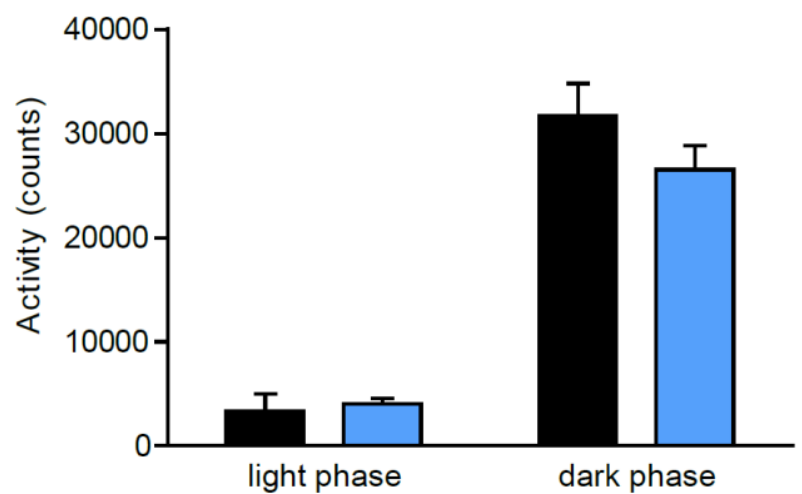

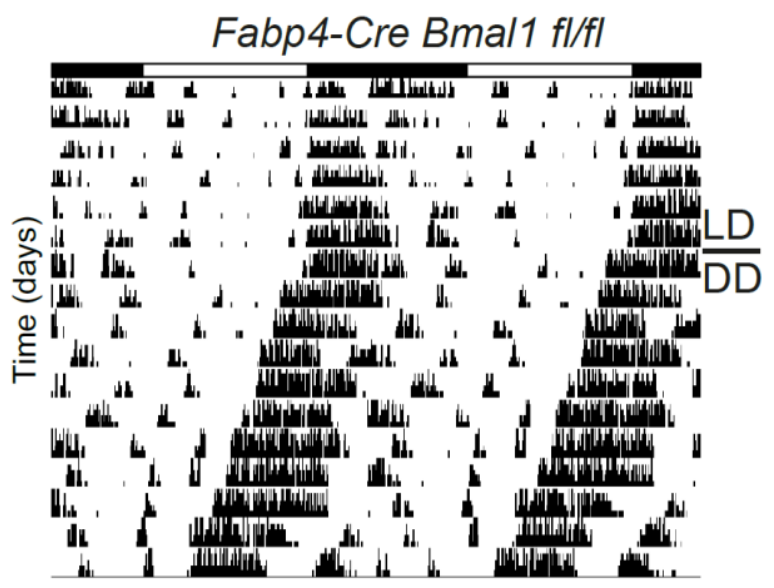

C

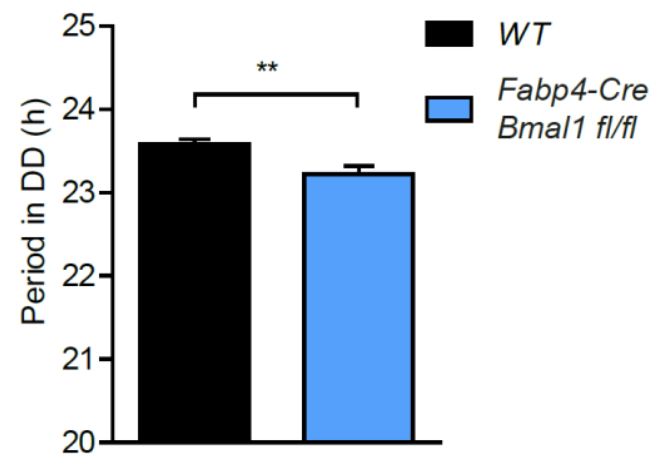

Figure 12. Behavioral characteristics of Fabp4-Cre Bmal1 fl/fl mice. (A) Representative actograms of wild-type and Fabp4-Cre Bmal1 fl/fl animals in LD (days 1-7) and DD (days 8-17). White and black bars indicate light and dark phases in LD. (B) Wheel-running activity during light and dark phases in LD averaged over 7 days ( $n=5-9)$. (C) Period of locomotor activity rhythms in DD as determined by $\chi^{2}$ periodogram analysis $(n=9-11)$. Data are presented as mean \pm SEM. ${ }^{* *}, \mathrm{P}<0.01$ by unpaired t-test.

\section{Fabp4-cre Bmal1 fl/fl mice show clock disruption in adipose tissue}

We focused our attention on epididymal adipose fat pads as the easiest to access and dissect type of WAT. To characterize local clock function in Fabp4-Cre Bmal1 $\mathrm{fl} / \mathrm{fl}$ animals, we performed qPCR quantification of clock gene expression under LD conditions. As expected Bmal1 transcript levels were strongly reduced in adipose tissue of mutant mice. We could, however, detect some residual Bmal1 mRNA, probably due to non-adipocyte cells which do not express CRE recombinase (Figure 13A)(Trayhurn, 2007). Similar results were obtained for Dbp, pointing at an adipocyte local clock disruption (Figure 13B). In contrast, Per2 expression levels remained strongly rhythmic, though 
amplitude was slightly reduced, consistent with its role as systemically driven gene (Figure 13C) (Kornmann et al., 2007).

A

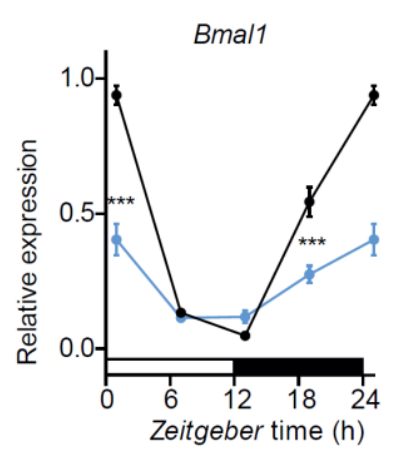

B

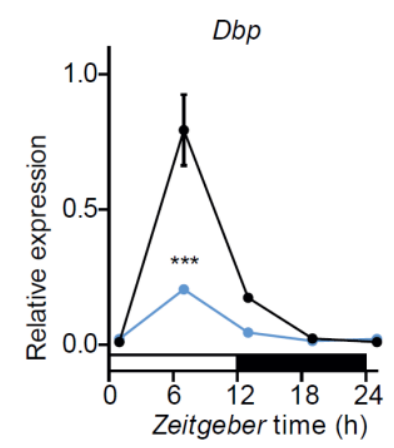

C

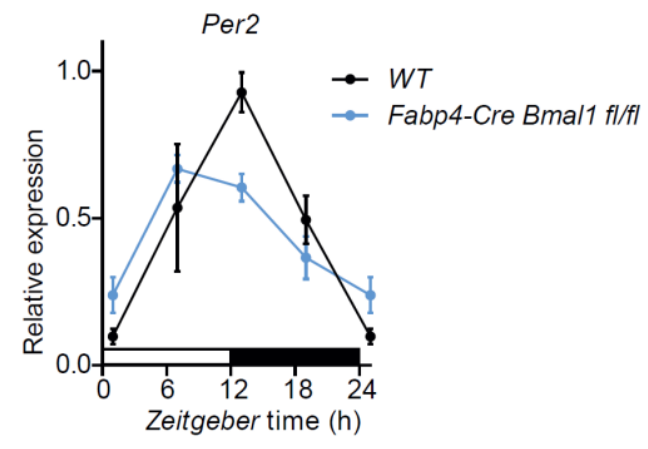

Figure 13. Disrupted circadian clock in WAT of Fabp4-Cre Bmal1 fl/fl mice. Expression profile of (A) Bmal1, (B) Dbp and (C) Per2 in epididymal fat pads of wild-type and Fabp4-Cre Bmal1 $\mathrm{fl} / \mathrm{fl}$ animals under LD conditions ( $\mathrm{n}=3-5$ per time point). Data are presented as mean \pm SEM. ${ }^{* *}, \mathrm{P}<0.01 ; * * *, \mathrm{P}<0.001$ by 2 -way ANOVA with Bonferroni post-test.

A

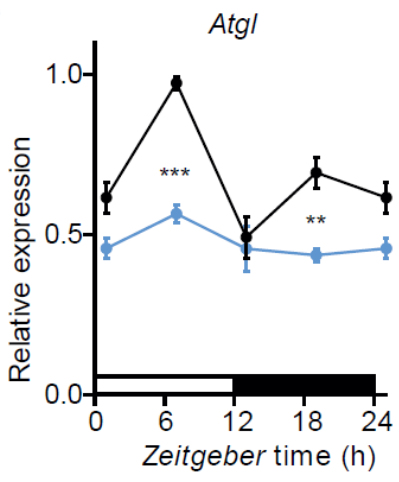

B

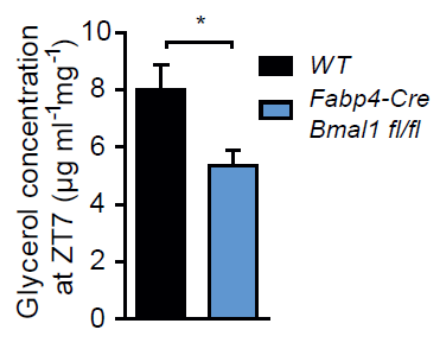

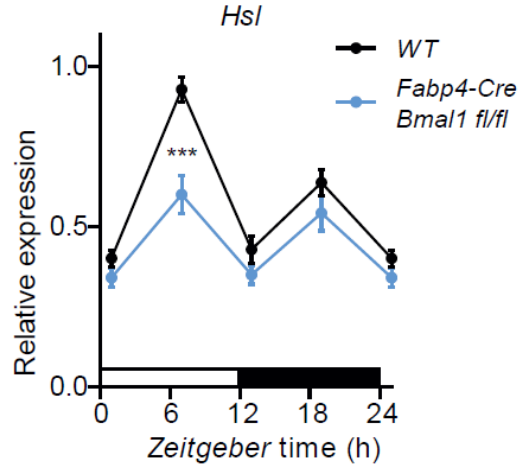

C

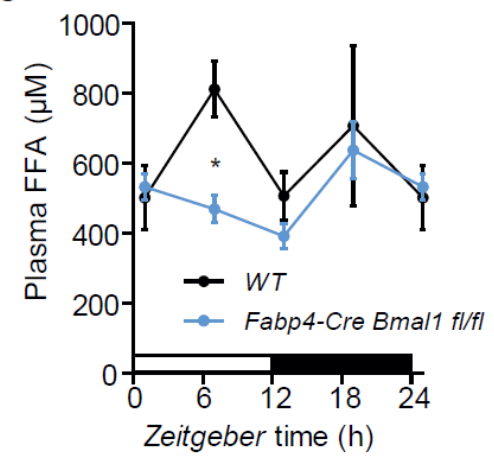

Figure 14. Lipolysis defects in adipose tissue of Fabp4-Cre Bmal1 fl/fl mice. (A) Expression profiles of Atgl and $\mathrm{Hsl}$ in WAT of mutants and wild-types in LD ( $\mathrm{n}=3-5$ per time point). (B) Glycerol excretion rates from fat pads of mutant and wild-type mice sacrificed at Zeitgeber time 7 ( $\mathrm{n}=10-16$; $^{*}, \mathrm{P}<0.05$, by unpaired $t$-test). (C) Diurnal FAA concentration profiles in plasma of Fabp4-Cre Bmal1 $\mathrm{fl} / \mathrm{fl}$ and control animals under LD conditions ( $\mathrm{n}=3-5$ per time point). Data are presented as mean $\pm \mathrm{SEM} .{ }^{*}, \mathrm{P}<0.05 ;{ }^{* *}, \mathrm{P}<0.01 ; * *, \mathrm{P}<0.001$ by 2 -way ANOVA with Bonferroni post-test. 
Fabp4-cre Bmal1 fl/fl mice show reduced lipolysis and low FFA concentrations in blood As suggested by our previous results, the circadian clock in adipose tissue transcriptionally controls lipolysis though CCGs like Atgl and Hsl. We decided to test this in Fabp4-Cre Bmal1 fl/fl mice. In line with previous data, qPCR revealed lower expression levels of Atgl and $\mathrm{Hs} /$ in adipose tissue of mutant mice when compared to wild-type controls (Figure 14A). We wondered whether this affects lipid mobilization in adipocytes and thus performed lipolysis assays with fat pads from Fabp4-Cre Bmal1 $f l / f l$ and control animals. Decreased glycerol excretion rates from WAT were observed in mutant fat pads at ZT7 (Figure 14B). In line with this, a marked reduction in FFA blood content in Fabp4-Cre Bmal1 fl/fl animals at ZT7 was observed (Figure 14C). These data strongly suggest that local adipose tissue clocks control lipid mobilization and WAT clock disruption leads to lower FFA availability in the blood.

A

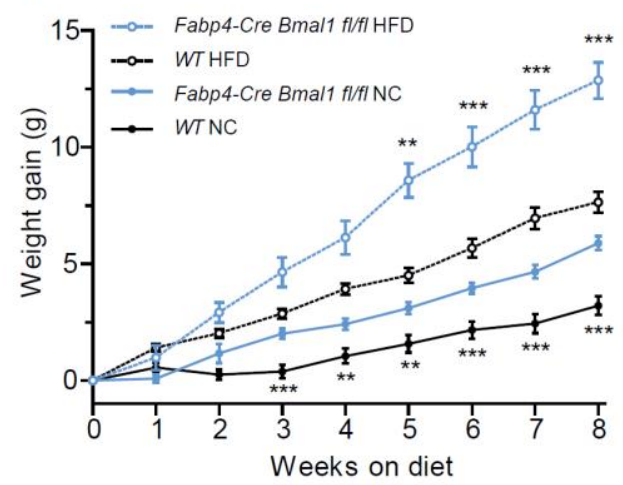

B

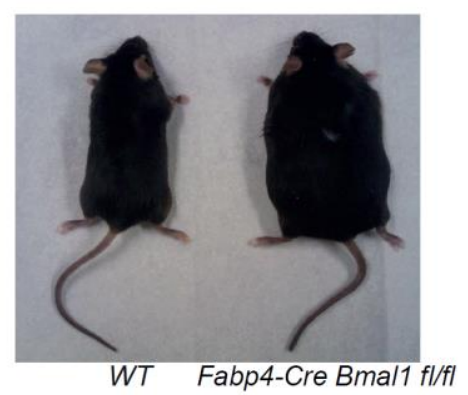

C

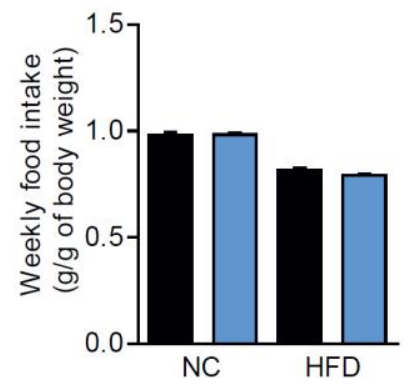

D

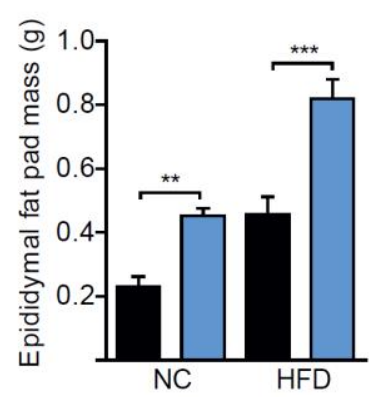

E

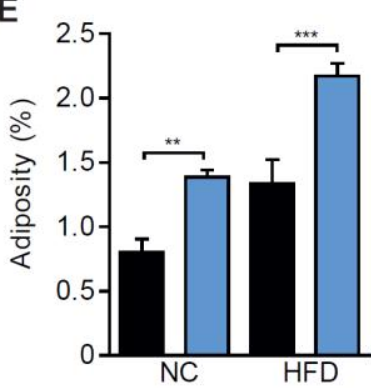

$\mathrm{F}$

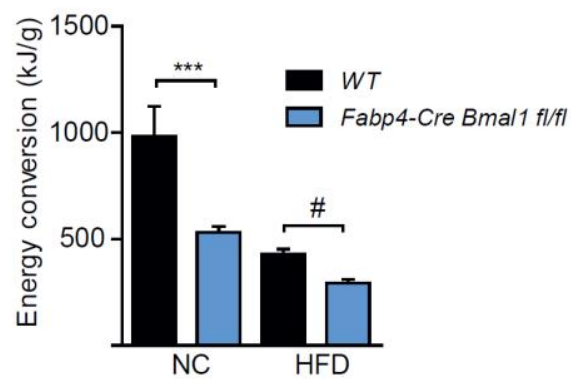

Figure 15. Fabp4-Cre Bmal1 fl/fl mice show higher body weight and increased adiposity. (A) Weight gain of mutants and wild-types during 8 weeks of HFD and NC ( $n=7-13)$. (B) Representative individuals of Fabp4-Cre Bmal1 $\mathrm{fl} / \mathrm{fl}$ and wild-type cohorts by the end of 8 weeks of HFD. (C) Food intake normalized to body weight ( $n=7-13)$, (D) epididymal fat pad weight ( $n=7-10),(E)$ adiposity $(n=7-10)$ and $(F)$ energy conversion on both diets $(\mathrm{n}=7-13){ }^{* *}, \mathrm{P}<0.01 ; * * *, \mathrm{P}<0.001$ by 2 -way ANOVA with Bonferroni post-test. $\#, \mathrm{P}<0.001$ by unpaired $t$ test. Data are presented as mean \pm SEM.

\section{Fabp4-cre Bmal1 fl/fl mice are obese}

Impaired lipolysis may provoke metabolic defects and growth of fat mass (Haemmerle et al., 2006). We studied this possibility challenging mutants and controls with different diet conditions. Under 45 $\%$ high-fat diet (HFD) conditions Fabp4-Cre Bmal1 fl/fl mice showed increased weight gain when 
compared to age-matched wild-type controls, with some of the animals reaching up to $45 \mathrm{~g}$ of bodyweight by the age of 17 weeks (Figure $15 \mathrm{~A}$ and B). Interestingly, at the same time mutants did not consume more food when normalized to body weight (Figure 15C). Similar results, albeit less pronounced, were also found under normal chow (NC) conditions (Figures $15 \mathrm{~A}$ and C). Under both diets Fabp4-Cre Bmal1 fl/fl mice showed a higher epididymal fat mass and adiposity (the ratio of epididymal fat mass to the total body weight), confirming that weight gain was largely attributed to increased fat content (Figures $15 \mathrm{D}$ and $\mathrm{E}$ ). Moreover, lower energy to body weight conversion rate also pointed at a highly anabolic phenotype (Figures $15 \mathrm{~F}$ ).

A

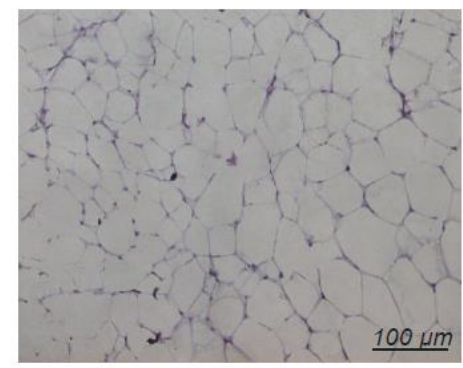

WT

B

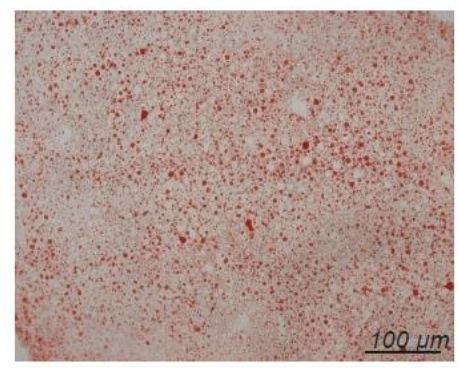

WT

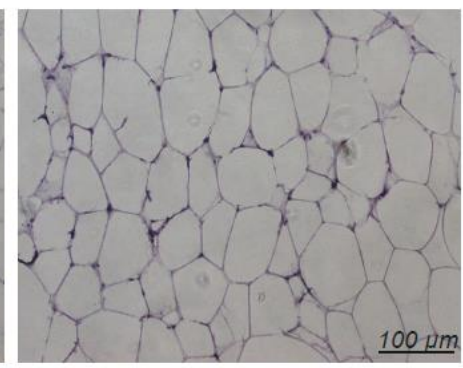

Fabp4-Cre Bmal1 fl/fl

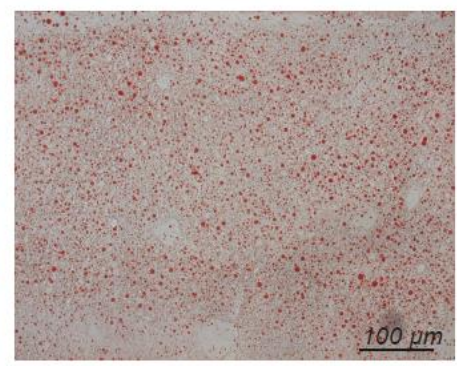

Fabp4-Cre Bmal1 fl/fl
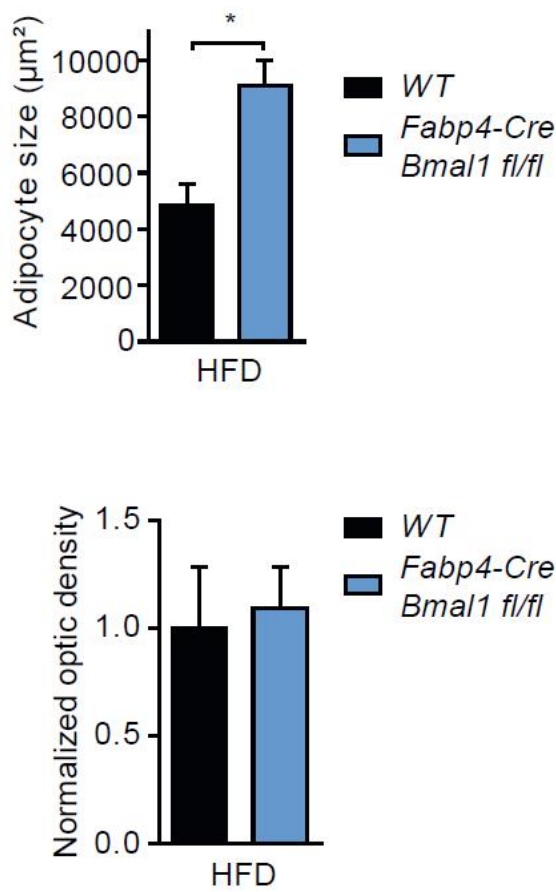

Figure 16. Histological analysis of WAT and liver. (A) Adipocyte hypertrophy and quantification of adipocyte size in epididymal WAT from Fabp4-Cre Bmal1 $\mathrm{fl} / \mathrm{fl}$ and control mice. (B) Oil Red O staining for triglyceride content of liver sections from mutant and wild-type animals $(n=4-5)$. Data are presented as mean \pm SEM. *, P $<0.05$ by unpaired $t$-test.

In order to reveal micro-structural changes in metabolic tissues of mutant mice we performed histological analysis of epididymal fat pads and liver. Consistent with data obtained from Clock $^{\Delta 19}$ mice, hematoxylin-eosin staining of adipose tissue showed increased adipocyte size suggesting triglyceride over-accumulation (Figure 16A). Remarkably, at the same time Oil Red O staining of liver sections did not reveal increased hepatic triglyceride deposition (Figures 16B). Of note, the observed phenotype was not correlated to hypoleptinemia since neither expression of leptin mRNA in adipose tissue nor its blood concentration were downregulated in weight-matched mutant animals (Figure 17 $A$ and $B)$. 
A

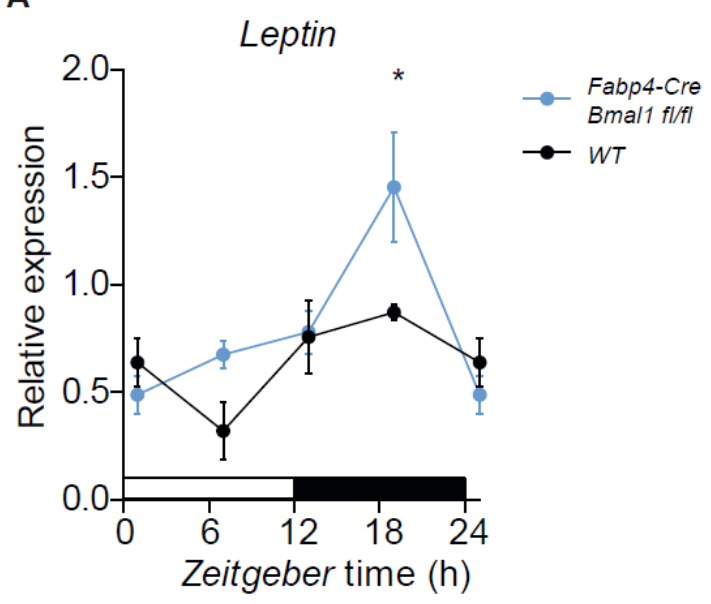

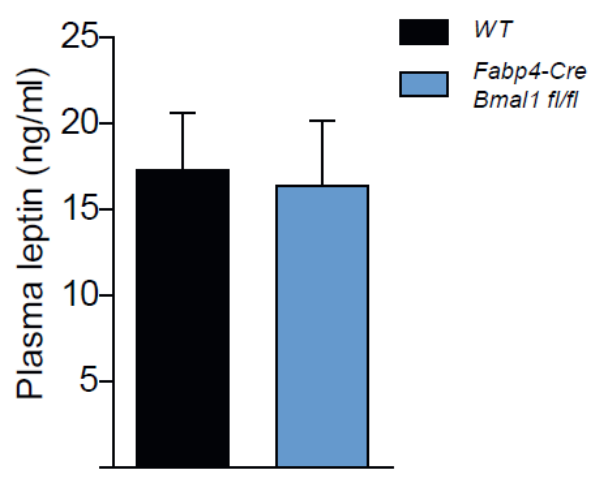

Figure 17. Circadian expression of Leptin mRNA ( $n=3-5$ per time point) (A) and plasma leptin concentrations $(\mathrm{n}=6-10)(\mathrm{B})$ in Fabp4-Cre Bmal1 $\mathrm{fl} / \mathrm{fl}$ and control mice. Data are presented as mean \pm SEM. *, P < 0.05 by 2 -way ANOVA with Bonferroni post-test.

\section{Falbp4-cre Bmal1 fl/fl mice exhibit a metabolic shift to carbohydrate utilization}

Animals with impaired lipolysis have reduced FFA blood content and thus utilize carbohydrates as primary energy source. The energy substrate usage can be monitored via measuring the respiratory exchange ratio (RER) using indirect calorimetry in metabolic cages. RER is a relation between produced $\mathrm{CO}_{2}$ and consumed $\mathrm{O}_{2}(\mathrm{VCO} 2 / \mathrm{VO} 2)$ and it reflects which fuel is being used to produce energy. An RER may vary from 0.7 (lipids are the predominant substrate) to 1.0 or higher (carbohydrates are the substrate). To test for diurnal changes in RER, we put Fabp4-Cre Bmal1 fl/fl mice in metabolic cages and measured their $\mathrm{CO}_{2}$ production and $\mathrm{O}_{2}$ consumption for several days. In agreement with previous studies, we could observe a clear substrate preference switch from fat during the inactive (light) phase to carbohydrates during the active (dark) phase in wild-type mice (Figure 18)(Satoh et al., 2006). In contrast, Fabp4-Cre Bmal1 fl/fl animals did not show such a change and during the light phase their RER remained high (Figure 18). Similar results were previously

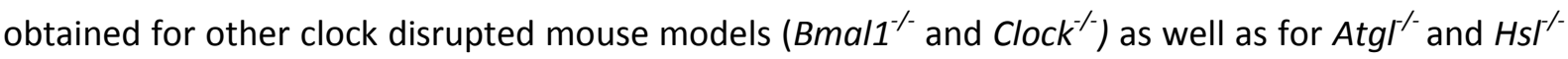
mutants, which also show impaired lipolysis (Eckel-Mahan et al., 2012; Huijsman et al., 2009; Shimba et al., 2011). Finally, we assessed the rhythmicity of food intake in Fabp4-Cre Bmal1 fl/fl mice under LD conditions. During the day mutants tend to eat more than controls yet the overall difference was not significant by 2-way ANOVA (Figure 19A). Moreover, mutant as well as wild-type animals showed a diurnal rhythm of food intake (Figure 19B). 


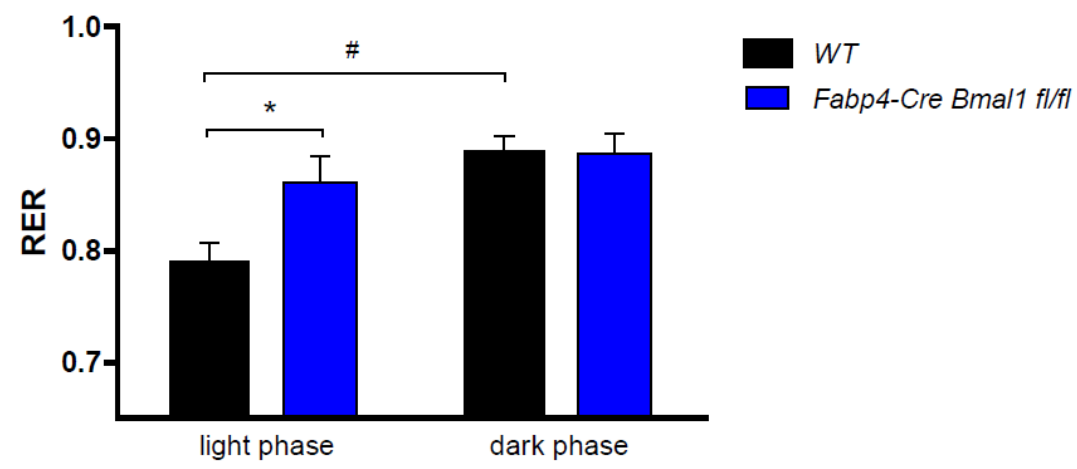

Figure 18. Respiratory exchange ratio (RER) of Fabp4-Cre Bmal1 fl/fl and control mice kept under LD ( $\mathrm{n}=4-5)$. Data are presented as mean \pm SEM. ${ }^{*}, \mathrm{P}<0.05$ by 2 -way ANOVA with Bonferroni post-test. ${ }^{\#}, \mathrm{P}<0.01$ by $t$-test. Experiment was performed by Dr. Olaf Jöhren.

A

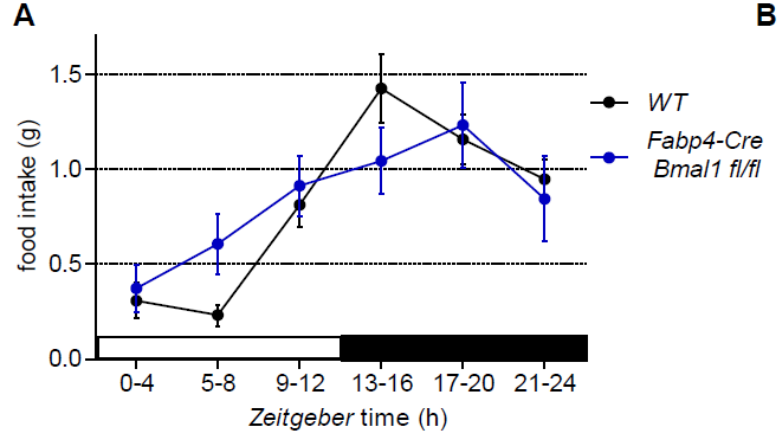

B

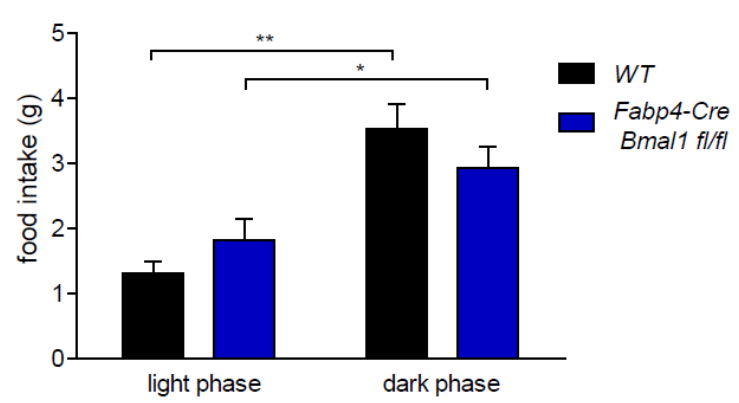

Figure 19. (A) Diurnal profiles and (D) day-night distribution of food intake of Fabp4-Cre Bmal1 fl/fl and control mice kept under LD ( $\mathrm{n}=4-5)$. Data are presented as mean \pm SEM. ${ }^{*}, \mathrm{P}<0.05 ;{ }^{* *}, \mathrm{P}<0.01$ by unpaired $t$-test. Experiment was performed by Dr. Olaf Jöhren. 


\section{Discussion}

In summary, the results of my thesis suggest that the circadian clock regulates lipid mobilization. In particularly, our data strongly argue that this function is attributed to local adipocyte oscillators that transcriptionally control two pacemaker lipolytic enzymes, Atgl and Hsl. Due to impaired lipolysis, white adipose tissues of clock gene mutant mice over-accumulate triglycerides. This work underpins an essential role for peripheral adipose clocks in the maintenance of metabolic homeostasis.

\section{A significant metabolic role for adipocyte clocks}

Using an ex vivo culturing technique, we demonstrated that adipocytes harbor self-sufficient circadian oscillators. While this suggests that adipose tissue is an integral part of the circadian system, it also raises the question which adipocyte functions may be affected by this local clock. To address this we concentrated our attention on triglyceride metabolism as a major aspect of adipose tissue physiology. Interestingly, some counterintuitive observations made from circadian clock gene mutant mice, such as low FFA - but not triglyceride - blood levels despite increased adiposity (Turek et al., 2005), already pointed to a potential involvement of adipose clocks in lipid trafficking. As we could find out, among the genes associated with triglyceride turnover many were potentially clockregulated targets, since they showed diurnal variations in transcription, which were abrogated in $\mathrm{Clock}^{\Delta 19}$ mutant mice. However, the down-regulation of most of these in $\mathrm{Clock}^{\Delta 19}$ adipose tissue could not explain the increase in adiposity observed in $\mathrm{Clock}^{119}$ mice. For instance, animals deficient for the phosphatidate phosphatase Lipin1 show progressive lipid dystrophy (loss of body fat) whereas mice, lacking long-chain-fatty-acid-CoA ligase 1 gene (Acs/1) in adipose tissues show normal body weight and fat content, even when kept on a high-fat diet (Ellis et al., 2010; Peterfy et al., 2001). Mice carrying loss-of-function mutations in genes responsible for FFA transport such as long-chain fatty acid transport protein 1 (Fatp1 or S/c27a1) and Calveolin 2 (Cav2) also show decreased or normal body weight, respectively (Razani et al., 2002; Wu et al., 2006). Deficiencies in genes associated with triglyceride de novo synthesis such as diglyceride acyltransferases 1 and 2 (Dgat1 and Dgat2) and 1acylglycerol-3-phosphate O-acyltransferase 2 (Agpat2) also result in lower body weight, reduced fat content or early postnatal death due to severe lipopenia (Dgat ${ }^{-1}$ ) (Smith et al., 2000; Stone et al., 2004; Vogel et al., 2011).

Thus, for further studies we focused on lipid mobilization since the expression of both pacemaker enzymes of lipolysis, Atgl and Hsl, was rhythmic in wild-type and arrhythmic and overall low in $\mathrm{Clock}^{\Delta 19}$ adipose tissue. We identified functional E-boxes in the promoter regions of both genes, which were inducible by CLOCK and BMAL1. Moreover the promoters of Atgl and Hs/ were bound by BMAL1 in vivo suggesting that both genes are under direct control of the circadian clock. But what 
does this circadian regulation mean for adipocyte physiology? First, we also could demonstrate that adipocyte clock function is necessary for rhythmic baseline lipolysis rates and circadian variations in the abundance of the lipolysis end products FFAs and glycerol in the blood. Second, circadian disruption results in increased triglyceride accumulation in WAT. Both of these phenomena we observed in $\mathrm{Clock}^{\mathrm{\Lambda 1}}$ and Bmal1\% mice (this thesis and (Guo et al., 2012; Turek et al., 2005)). Thus, clock gene deficient animals recapitulate the phenotype of mutants with impaired lipolysis such as Atg ${ }^{-/}$mice (Haemmerle et al., 2006). FFAs become a major energy source during periods of fasting (including the diurnal rest phase, i.e. the night in humans and the day in mice). At the same time an excess of FFAs in the blood is pernicious since FFAs can disrupt the integrity of biological membranes and exert many other deleterious effects termed lipotoxicity (Unger et al., 2010). Thereby FFA concentrations have to be tightly controlled and the circadian gating of their release from WAT is reasonable (Figure 20). In mice FFA levels are high during the day when the animals are normally inactive and eat very little, whereas, during the active phase, mice rely largely on carbohydrates and lipids from ingested chow. During that time adipose-derived FFA levels are low.

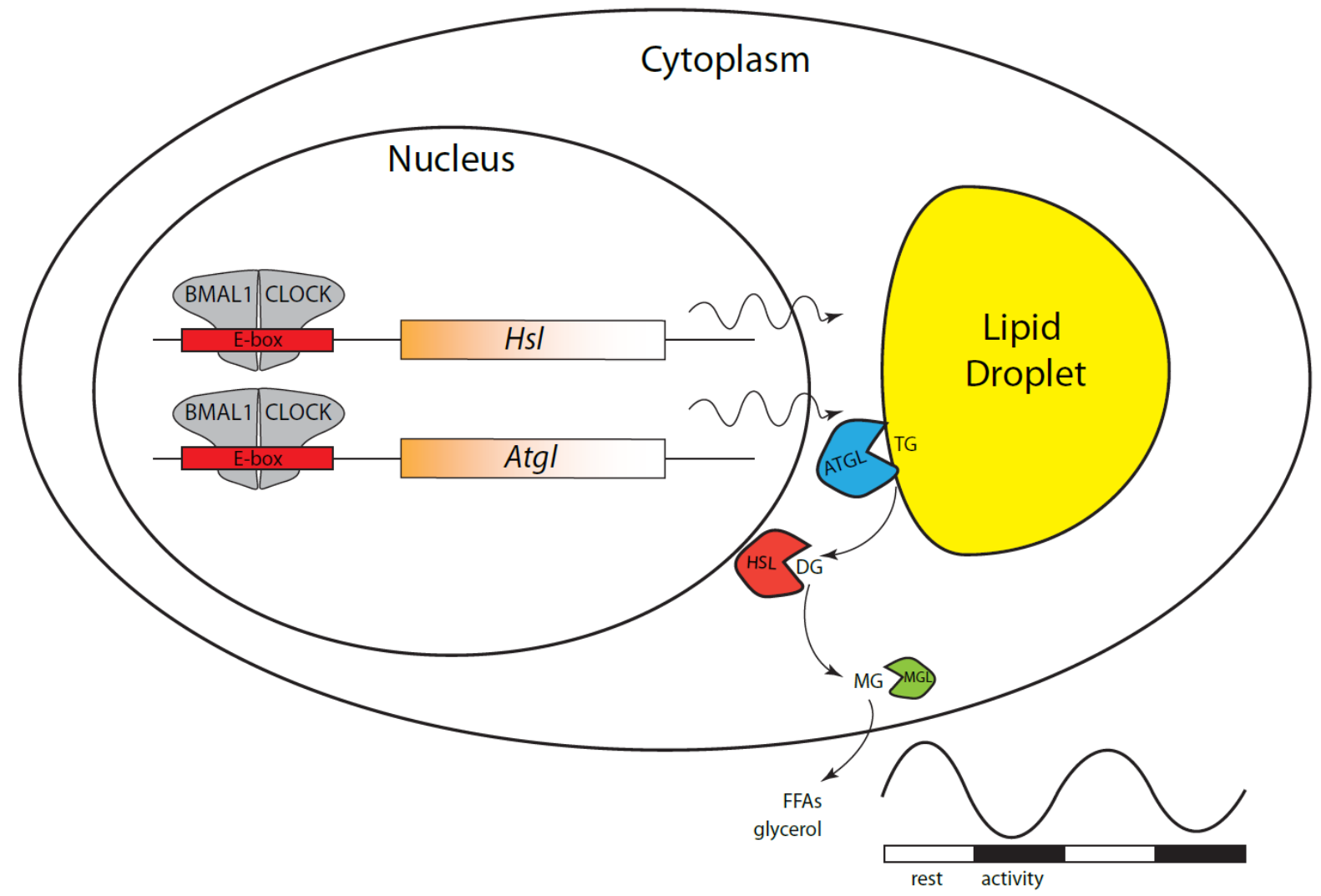

Figure 20. A graphical summary of the circadian regulation of basal adipocyte lipolysis. Circadian transcriptional regulation of Atgl and $\mathrm{Hsl}$ regulates hydrolysis of triglycerides (TG) to diacylglycerols (DGs) and monoacylglycerols (MGs). This leads to a circadian rhythm of glycerol and FFA levels in the blood.

Another manifestation of impaired lipolysis is decreased tolerance to prolonged fasting (Wu et al., 2012). To test whether circadian mutant mice also show such a disturbance, we starved Clock ${ }^{\Delta 19}$ 
animals for 1.5 days. During this period Clock ${ }^{\Delta 19}$ mutants showed drastically decreased blood concentrations of both glycerol and FFAs. Fasting induces activity of ATGL and HSL via phosphorylation by PKA, thus overriding circadian effects in basal lipolysis (Pagnon et al., 2012; Wu et al., 2012). We hypothesized that WAT of $\operatorname{Clock}^{\Delta 19}$ mice have impaired response to $\beta$-adrenergic lipolysis induction. Indeed, upon stimulation with isoproterenol $\mathrm{Clock}^{119}$ fat pads showed slightly reduced lipolysis rates than wild-types. During fasting we also observed a drop in body temperature as the result of decreased FFA oxidation in brown adipose tissues. To compensate for the lack of lipid fuel under fasting conditions $\mathrm{Clock}^{\Delta 19}$ mutants presumably actively utilize glucose. In line with this their liver glycogen stores were rapidly depleted. These results do not agree with previous observations that liver glucose export is subjected to circadian control via transcriptional control of Glut2 and therefore blood glucose levels should also be reduced in Clock ${ }^{\Delta 19}$ mice (Lamia et al., 2008). Low blood glucose could also reflect high glucose utilization by other tissues such as muscle. Alternatively, under fasting conditions lower GLUT2 levels could be compensated by an increase in Glut1 expression (Thorens et al., 1990)

\section{Feeding vs, clock regulation of lipolysis}

Another important aspect of circadian gene expression in peripheral tissues are systemic cues (blood, autonomic innervation, body temperature, feeding) coming from the body. Kornmann et al. showed that upon tissue-specific disruption of the liver clock by conditional Rev-erbo overexpression in hepatocytes a subset of clock and clock-regulated genes (e.g. Per2 and Noc) do not show changes in their circadian expression profiles (Kornmann et al., 2007). This suggested that while expression of most rhythmic genes is regulated by local clocks, systemic signals can drive rhythms of others (Kornmann et al., 2007). Such a systemic signal could be feeding since it is a major entraining signal for peripheral clocks. Under temporally restricted feeding conditions metabolic organs such as liver and adipose tissue exhibit a phase-shift of clock gene expression independent of the SCN (Damiola et al., 2000; Zvonic et al., 2006). Moreover, restricted food availability can rescue rhythmic transcription of many genes in clock-deficient $\mathrm{Cry} 1^{--} \mathrm{Cry}^{2--}$ mice, suggesting that these transcripts are rather fooddriven than directly controlled by the clock (Vollmers et al., 2009). Therefore blunting the day-night variations in food intake seen in circadian mutants may reduce the rhythmicity of many output genes and possibly of Atgl and Hsl (Turek et al., 2005). To test if local clock function is sufficient for rhythmic regulation of $\mathrm{Atgl}$ and $\mathrm{Hs} /$ we utilized ex vivo fat pad culturing in order to eliminate any rhythmic signal from the body. This way we could confirm that circadian expression of $\mathrm{Atgl}$ and $\mathrm{Hsl}$ is regulated by local adipose tissue clocks. Similar results were also obtained from synchronized NIH3T3 fibroblasts transfected with Atgl-luc and Hsl-luc reporter constructs. Moreover, In humans rhythmic food intake is not required for circadian lipolysis since under constant routine conditions (enforced posture, constant dim light, hourly isocaloric meals, and sleep deprivation) blood levels of 
glycerol and FFAs still show circadian variations (Dallmann et al., 2012). However, apart from CLOCK and BMAL1, Atgl and $\mathrm{Hsl}$ are subjected to direct transcriptional control by food regulated transcription factors such as PPARy , IRF4 and USF1/2 (Eguchi et al., 2011; Kershaw et al., 2007; Kim et al., 2006; Smih et al., 2002). It is also worth to mention that some circadian processes within adipocytes might be regulated by rhythmic humoral and neuronal signals. In this study we measured basal lipolysis rates which reflect the expression of Atgl and Hsl (Ryden et al., 2007). At the same time lipolysis is post-transcriptionally regulated by hormones such as leptin, noradrenalin and insulin which also can transmit temporal information (Duncan et al., 2007). Thus, we cannot fully exclude that feeding plays a role in regulating lipolysis in vivo and the systemic input from the rest of the body cannot be neglected.

\section{Adipocyte Bmal1 deficient mice}

Bmal1 is the only non-redundant clock gene in the mammalian $T T L$, evidenced by the finding that global and SCN-specific deletion of Bmal1 produces an arrhythmic behavioral phenotype in mice (Bunger et al., 2000; Husse et al., 2011). To analyze the contribution of Bmal1 to peripheral clock regulation Storch and colleagues produced a transgenic mouse line carrying a Bmal1 gene flanked by two loxP sites (Storch et al., 2007). When this line is crossed with mice expressing CRE DNA recombinase under control of a tissue-specific promoter, this results in Bmal1 gene deletion and ultimately leads to clock disruption in the given tissue. This approach was already used to generate liver-, macrophage- and brain-specific clock knock-out mice (Gibbs et al., 2012; Lamia et al., 2008; Mieda and Sakurai, 2011). For analyzing adipocyte clock function we selected the Fabp4-Cre driver line as a frequently used model for targeted recombination in adipose tissues (He et al., 2003). Indeed, we could observe a strong reduction of BMAL1 protein and Bmal1 transcript in adipose tissue of Fabp4-Cre Bmal1 fl/fI mice, which abolished local clock function. We also checked BMAL1 levels in other tissues in order to exclude ectopic recombination. Although liver and muscles did not show any changes, BMAL1 levels in many brain areas were strongly reduced. Nonetheless, central pacemaker function seemed only marginally affected since the expression of clock genes in the SCN was not significantly altered, albeit Fabp4-Cre Bmal1 $\mathrm{fl} / \mathrm{fl}$ mice showed some alterations at the behavioral level. The SCN controls activity indirectly, predominantly via secretion of neuropeptides, and the target of their action is largely unknown (reviewed in (Welsh et al., 2010)). Thus it is likely that behavioral defects in Fabp4-Cre Bmal1 $\mathrm{fl} / \mathrm{fl}$ mice may stem from Bmal1 deficiency in brain regions downstream of the $\mathrm{SCN}$ that affect the circadian rhythms of locomotor behavior.

In WAT of Fabp4-Cre Bmal1 fl/fl mutants, and in agreement with our data from Clock ${ }^{\Delta 19}$ and $\mathrm{Bmal1}^{-/}$ mice, Atgl and $\mathrm{Hsl}$ expression rhythms were blunted and low. This correlated with a reduction of lipolysis rates and FFA levels in the blood, supporting that adipose tissue clocks are regulators of lipid 
mobilization. Surprisingly, Fabp4-Cre Bmal1 fl/fl animals also showed similar metabolic abnormalities as Clock $^{\Delta 19}$ mutants. They were heavier than wild-types when fed regular chow and developed morbid obesity under HFD conditions. Fabp4-Cre Bmal1 $\mathrm{fl} / \mathrm{fl}$ mice had double the amount of epididymal fat due to highly hypertrophic adipocytes. In contrast to our results, cell-based experiments suggest that Bmal1 is required for late stages of adipocyte differentiation, while during early stages Bmal1-defficient pre-adipocytes accumulate more triglycerides than control cells (Guo et al., 2012; Shimba et al., 2005). Nevertheless, both $\mathrm{Bmal}^{-1}$ and $\mathrm{Clock}^{\Delta 19}$ animals show metabolic syndrome symptoms and higher adiposity, though mature $\mathrm{Bmal1}^{-1}$ mice progressively loose adipose tissue due to premature aging (Guo et al., 2012; Kondratov et al., 2006; Turek et al., 2005). Therefore there is certain inconsistence between in vivo and in vitro data, probably due to pleotropic effects of Bmal1 deficiency, and these phenomena need to be addressed in future studies. We did not see any increased lipid accumulation in livers of Fabp4-Cre Bmal1 $\mathrm{fl} / \mathrm{fl}$ mice though as was reported for the $\mathrm{Bmal1}^{\%}$ mutants (Shimba et al., 2011). We suppose, this effect may be caused by disruption of the liver clock in conventional Bmal1 deficient model, while in our conditional mutants the clock in hepatocytes was intact.

Similar to $\mathrm{Clock}^{\Delta 19}$ and $\mathrm{Bmal1}^{--}$mice, Fabp4-Cre Bmal1 $\mathrm{fl} / \mathrm{fl}$ mutants experienced a state of FFA deficiency and mostly utilized glucose as energy source as indicated by elevated RER values during the rest phase. We could observe a slightly attenuated feeding rhythm in Fabp4-Cre Bmal1 fl/fI mice in comparison to wild-type animals, yet the overall food intake was not different between both genotypes. On could speculate that in addition to central regulation this effect might in part be triggered by lower amount of FFAs in the blood during the resting phase. Indeed, it is conceivable, that by the end of the day both Fabp4-Cre Bmal1 $\mathrm{fl} / \mathrm{fl}$ and $\mathrm{Clock}{ }^{\Delta 19}$ mutants suffer from energy deficiency and have to resume eating before the end of their normal sleeping times. Alternatively, FFAs can signal to the CNS in order to regulate appetite and leptin sensitivity via Toll-like receptor 4 (TIr4), thus disruption of FFA rhythms could interfere with central leptin signaling (Kleinridders et al., 2009). Some studies proposed that disruption of feeding rhythmicity per se, which is analogous to night eating syndrome in humans, is responsible for the weight gain in clock deficient mice (Hatori et al., 2012; Kohsaka et al., 2007; Turek et al., 2005). On the other hand, not all arrhythmic mouse models show increased body weight, in particular Per $2^{--}$and $\mathrm{Cry} 1^{-} \mathrm{Cry} 2^{--}$mutants are known to be lean (Bur et al., 2009; Grimaldi et al., 2010). However one question remains: what metabolic consequence has the reduction of BMAL1 protein in the brain of Fabp4-Cre Bmal1 $\mathrm{fl} / \mathrm{fl}$ mice? A previous study provided evidence that mice with brain-specific Bmal1 deletion (Nestin-Cre Bmal1 fl/-) do not show increased body weight or food intake. This observation becomes even more remarkable considering the fact that in the brain of Nestin-Cre Bmal1 $\mathrm{fl} /$ - mice appetite-regulating regions such as dorsal medial nucleus (DMH) and arcuate nucleus show a reduction of Bmal1 of up to $80 \%$ (Mieda 
and Sakurai, 2011). However, it still possible that some aspects of the observed metabolic phenotype of Fabp4-Cre Bmal1 $\mathrm{fl} / \mathrm{fl}$ animals originates from Bmal1 recombination in the brain. More specific genetic tools, such as the newly-developed adipocyte-specific Adiponectin-Cre line, may help to address this issue in the future (Eguchi et al., 2011).

\section{Physiological importance of peripheral clocks}

A major role of circadian clocks is to provide an anticipation of up-coming daily changes and thereby optimize the performance of the organism (Green et al., 2002). In addition, the body clock helps to maintain synchrony among myriads of biochemical processes which need to be compartmentalized from each other both spatially and temporally (Harmer et al., 2000). This is particularly important for animals with their complex behavior and multi-organ composition of the body. In mammals cellular self-sufficient circadian oscillators are found throughout the body organized in a defined hierarchy (Nagoshi et al., 2004; Yoo et al., 2004). However, once reset population rhythms of peripheral cells eventually dampen due to gradual desynchronization of sustained oscillations of individual cells (Welsh et al., 2004). Thus, to govern this complexity the central nervous system developed a special structure - the master oscillator located in the SCN. SCN neurons obtained a superior property of the intercellular coupling which allowed them to generate robust circadian oscillations and maintain tight synchronization of the organ (Liu et al., 2007; Yamazaki and Takahashi, 2005). Moreover owing to the direct retinal innervation, the SCN can incorporate external light-dark information and convey it to peripheral oscillators (Welsh et al., 2010). Despite the lack of such direct light input and coupling, tissue clocks are important for the maintenance of physiology and energy homeostasis. According to the current model, circadian oscillators in peripheral tissues regulate organ-specific functions by driving physiological reactions at particular times (e.g. liver - glucose export, pancreas - insulin secretion, heart -repolarization rates) (Jeyaraj et al., 2012; Lamia et al., 2008; Marcheva et al., 2010). Although genome-wide transcriptome profiling studies show that approximately $12 \%$ of all genes exhibit diurnal variations in transcription, there is just a small portion of overlapping cyclic genes common between different tissues (Panda et al., 2002; Storch et al., 2002). Thus, local circadian clocks exert their control of via extensive rhythms of gene transcription and the identification of genes which connect timing mechanisms with physiologically meaningful output represents a novel challenge for circadian biology (reviewed in (Dibner et al., 2010)).

In order to stabilize the circadian system, the master clock can integrate hormonal and neuronal feedback from peripheral oscillators. Humoral factors such as androgens or estrogens can modulate clock gene expression in the SCN and affect the period of locomotor behavior (Karatsoreos et al., 2007; Morin et al., 1977; Nakamura et al., 2005). In addition brain peripheral oscillators in arcuate 
and raphe nuclei may relay metabolic information to the $\mathrm{SCN}$ through direct innervation (Malek et al., 2007; Yi et al., 2006).

In summary, the circadian organization in the body appears to follow a standard top-down paradigm: central clock - peripheral clocks - physiological reactions. A large-scale network of organ clocks creates a multilevel highly labile structure which can be entrained by various Zeitgebers. Importantly such system can simultaneously incorporate temporal information from different external cues, such as the light-dark cycle and food, even in case when they are in anti-phase from each other (Damiola et al., 2000). Thus, complex hierarchal organization of the circadian system confers plasticity and stability against perturbations.

\section{Conclusions \& outlook}

\section{White spots in adipose circadian biology}

We have demonstrated that one of the functions of adipose tissue clocks is the circadian regulation of lipolysis. In WAT there are many other pathways which show circadian variation and thus are potentially controlled by local clocks (Zvonic et al., 2006). For example, with the exception of leptin, we have not yet studied the circadian control of adipokine expression, which may have a substantial contribution to metabolic regulation. Moreover it is very likely that through humoral signaling peripheral clocks can feedback to the brain (and maybe the SCN) in order to modulate behavior in response to energy requirements. Adipocyte clock-deficient mice will be useful to study these questions.

Nowadays, there is a growing understanding of the function of peripheral circadian clocks in physiological regulation (Albrecht, 2012). While our study demonstrates such a function for adipose tissue clocks, the physiological meaning of many other peripheral clocks in different organs as well as particular brain structures still remains unclear. For instance, the function of circadian clocks within brown adipose tissue (BAT) has not been studied and we show that ex vivo cultured BAT explants exhibit circadian gene expression. Brown adipocytes are important for heat production via intensive FFA oxidation, which also might be subject to circadian control (Ravussin and Galgani, 2011; Redlin et al., 1992; Zvonic et al., 2006). With development of suitable transgenic mouse models the physiological impact of BAT clock disruption can be assessed.

\section{Implications of circadian clocks in human metabolism}

Studies conveyed on laboratory animals explicitly demonstrate that disruption of circadian rhythms can lead to obesity and metabolic disorders. Epidemiological human studies also provide evidence that synchrony between the light-dark cycle, sleep and eating is required for normal function of human physiology (reviewed in (Froy, 2010)). Indeed, short sleep duration is associated with 
increased body mass index (BMI) and higher incidence of type 2 diabetes (Gottlieb et al., 2005; Taheri et al., 2004; Vorona et al., 2005). Sleep loss increases appetite reducing leptin blood levels and induces visfatin rhythm phase shifts which correlate with elevated blood glucose (Benedict et al., 2012; Spiegel et al., 2004). Another problem of modern society - shift work - is also associated with a higher incidence of cardiovascular disease, obesity and metabolic syndrome (Ellingsen et al., 2007; Karlsson et al., 2001).

In addition to environmental factors, naturally occurring genetic polymorphisms of clock genes in humans predisposes to metabolic abnormalities. Certain haplotypes of CLOCK and BMAL1 genes were shown to increase the susceptibility to obesity, type 2 diabetes and hypertension (Scott et al., 2008; Sookoian et al., 2008; Woon et al., 2007). Genetic variants of PER2 and NPAS2 are associated with high fasting blood glucose and hypertension, respectively (Englund et al., 2009). Genetic links to obesity were also demonstrated for some clock regulated adipokines such as NAMPT (visfatin) (Blakemore et al., 2009).

Adipose tissue clocks potentially represent a novel target for the pharmacological manipulation. While the SCN clock is very difficult to reach due to its localization, peripheral clocks can be treated by various agonists and antagonists of clock proteins such as REV-ERB $\alpha / \beta$ and $C R Y 1 / 2$ in order to adjust metabolic state of the body (Hirota et al., 2012; Solt et al., 2012).

Given the tight association of clock and metabolism, the comprehension of circadian aspects of metabolic regulation become critical against the background of an increasing prevalence of obesity in the modern world. Although obesity is an extremely complex disorder, it is clear that bad dietary habits and non-regular feeding and sleep schedules are the major contributors. The understanding of the function of peripheral clocks will help us to disentangle the complex system of circadian and metabolic interconnections and, thus, will provide novel insights into the circadian component of obesity development. 


\section{Material and Methods}

\section{Wheel running analysis}

Wheel running experiments were carried out as described previously (Jud et al., 2005). For all behavioral experiments male mice between 2 and 6 months of age were used. During recording, mice were kept in individual transparent plastic cages equipped with a running-wheel. Wheel revolutions were detected via a magnetic switch connected to a computer system. 12 cages were kept in one isolation chamber, in which lights were controlled via a computer system. Temperature $\left(20 \pm 0.5^{\circ} \mathrm{C}\right)$ and humidity $(50-60 \%)$ were kept constant. Animals with different genotypes were always distributed equally between different isolation cabinets. Mice were provided standard chow food (Ssniff V1126) and water ad libitum. Locomotor activity parameters were analyzed using the ClockLab analysis software plug-in (Actimetrics) for MatLab (The Mathworks).

\section{Tissue and blood collection}

Animals were sacrificed at the indicated time points by cervical dislocation. Mice which had to be sacrificed during the dark phase were handled under red light and eyes were removed before dissection of tissues to prevent acute light effects on gene expression (Albrecht et al., 1997). To prepare serum, blood was collected and allowed to clot on ice. After centrifugation for 20 min at $2,000 \mathrm{~g}\left(4^{\circ} \mathrm{C}\right)$, supernatants were collected in fresh tubes. For plasma isolation trunk blood was collected at ZT 1, 7, 13, and 19 in EDTA-containing tubes (Sarstedt) and centrifuged at 2,000 g for 20 min. Serum/plasma samples were stored at $-80^{\circ} \mathrm{C}$. Collected tissue samples were immediately frozen in liquid nitrogen and transferred to $-80{ }^{\circ} \mathrm{C}$ for long-term storage. Plasma FFA and leptin levels were determined using NEFA kit (Zen-Bio) and Leptin ELISA kit (Cristal Chem inc.) according to the manufacturer's protocol.

\section{Luminescence measurement}

Luminescence measurements were performed with a LumiCycle luminometer (Actimetrics). Standard settings for the LumiCycle were:

O Integration time: $75 \mathrm{sec}$

○ Measurement interval: $10 \mathrm{~min}$

The data from Per2::luc mice was analyzed with the LumiCycle analysis program (Actimetrics) (Figure 21). The raw data were baseline subtracted using a running average of $24 \mathrm{hrs}$. 


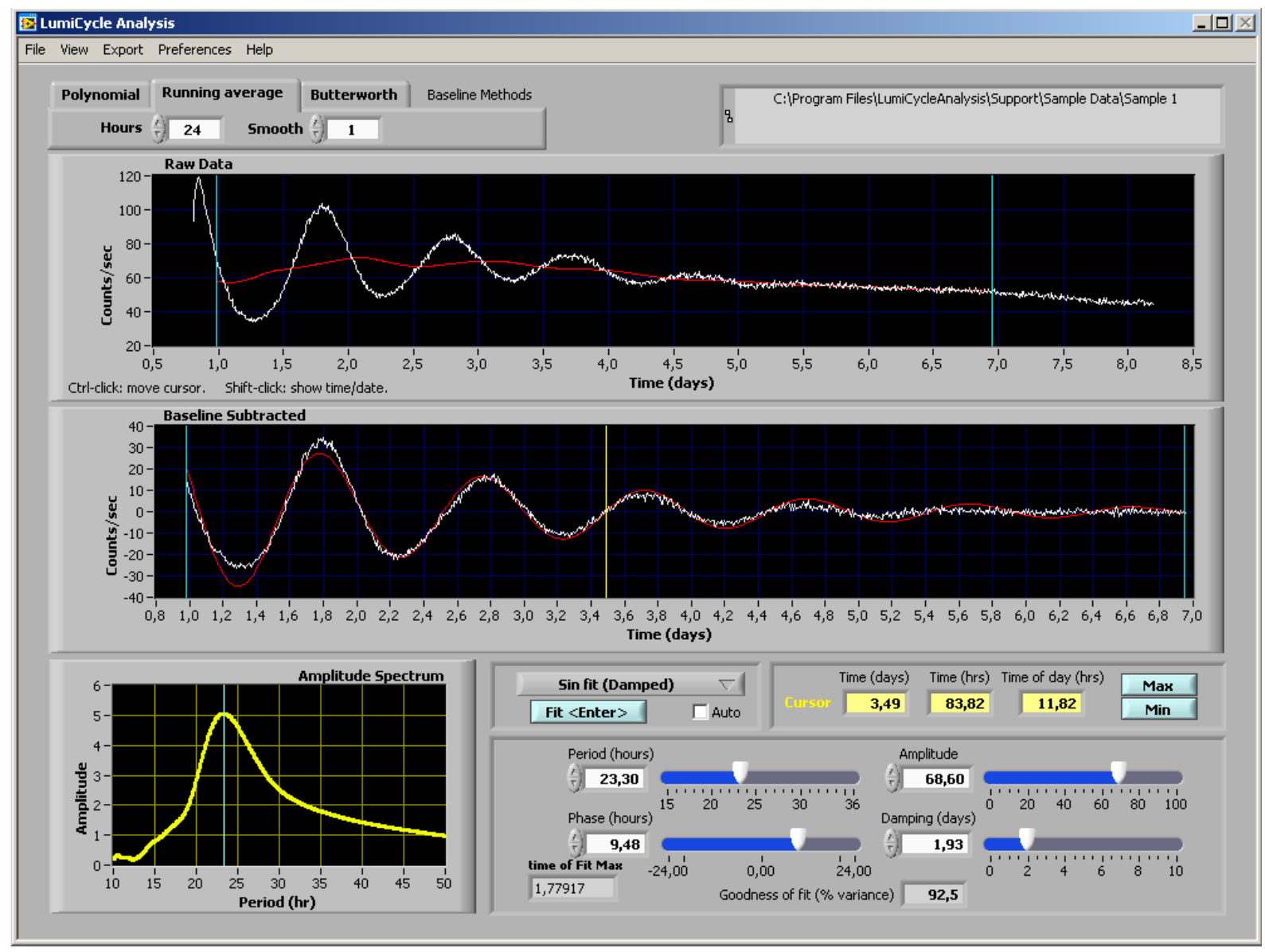

Figure 21 . The interface of the Actimetrics LumiCycle software. The upper and the lower panels show raw data and baseline subtracted data, respectively. The peak time can be defined using the moveable yellow bar in the baseline subtracted data panel. The software also allows determining other parameters of oscillations such as period (h), amplitude, phase (h) and damping (days).

\section{Histological methods}

\section{Oil Red 0 staining}

Cryosections $(8-10 \mu \mathrm{m})$ were fixed in $4 \%$ PFA for $20 \mathrm{~min}$. Saturated (approximately $1 \%$ ) stock solution of Oil Red O (Sigma-Aldrich) in $99 \%$ isopropanol was prepared at least 2 days before staining. $1 \%$ dextrin solution (Sigma-Aldrich) was prepared the day before and stored at RT overnight, then was filtered through filter paper. The working solution was prepared by mixing $60 \mathrm{ml}$ of stock solution with $40 \mathrm{ml}$ of $1 \%$ dextrin and filtered through filter paper. Frozen sections were stained $20 \mathrm{~min}$ in Oil Red $\mathrm{O}$ staining solution, and then washed in $60 \%$ isopropanol. Sections were mounted with glycerin jelly prepared as follows: $1 \mathrm{~g}$ of gelatin in $6.5 \mathrm{ml}$ distilled water was heated and $7 \mathrm{ml}$ glycerol was added and mixed. The images were analyzed using the ImageJ software.

\section{Hematoxylin-eosin staining}

Deparaffinization and rehydration of sections:

- $2 \times 5$ min xylene,

○ $2 \times 5 \min 100 \%$ ethanol, 
○ $2 \times 5 \min 95 \%$ ethanol,

- $5 \min 80 \%$ ethanol,

- 5 min deionized water.

While sections were in water, hematoxylin was filtered through filter paper (Whatman). Excess water was blotted from the slide holder before hematoxylin staining.

Hematoxylin staining:

○ 3 min hematoxylin,

$\circ$ rinsing with deionized water,

- 5 min tap water,

- dipping 8-12 times into acid ethanol ( $1 \mathrm{~mL}$ concentrated hydrochloric acid in $400 \mathrm{ml} 70 \%$ ethanol),

- rinsing with tap water $(2 \times 1 \mathrm{~min})$,

$\circ$ rinsing with deionized water $(2 \mathrm{~min})$,

- excess water was blotted from the slide holder before eosin staining.

Eosin staining and dehydration:

- $30 \mathrm{sec}$ eosin (1 $\mathrm{g}$ eosin $\mathrm{Y}$ in $100 \mathrm{ml}$ deionized water),

- $3 \times 5 \min 95 \%$ ethanol,

- $3 \times 5$ min $100 \%$ ethanol,

○ $3 \times 15$ min xylene,

- slides were mounted with a xylene-based mounting medium (Permount).

\section{Immunohistochemistry with antiBMAL1 antibodies}

Brains were embedded in OCT medium (Tissue-Tek) and $10 \mu \mathrm{m}$ sections were cut using a cryotome (Leica). Sections were pre-treated with $3 \% \mathrm{H}_{2} \mathrm{O}_{2}$ in methanol for $10 \mathrm{~min}$ at room temperature and washed 3 times with deionized water. Antigen retrieval was performed in $0.01 \mathrm{M}$ sodium citrate (pH 6.0) for $2 \mathrm{~min}$ at room temperature. Then sections were boiled in the same solution for $5 \mathrm{~min}$ and cooled on ice for $20 \mathrm{~min}$. Next, slides were washed 2 times with TNT buffer $(0.1 \mathrm{M}$ Tris- $\mathrm{HCl} \mathrm{pH}$ 7.5, $150 \mathrm{mM}$ sodium chloride, $0.05 \%$ TWEEN 20) for 2 min each. Blocking solution was applied for 2 $\mathrm{h}$ at room temperature (Vectastain kit) followed by BMAL1 antibody solution (1:1,000 in TNT buffer) at $4{ }^{\circ} \mathrm{C}$ overnight. On the next day sections were washed in TNT buffer for $5 \mathrm{~min}$, twice in PBS for 5 min and covered with secondary antibody (Vectastain kit) solution for $20 \mathrm{~min}$. After 5-min PBS wash the $A B C$ reagent (Vectastain kit) was applied for $30 \mathrm{~min}$. Then the $A B C$ reagent was removed and slides were washed with PBS for 5 min and the DAB reagent (Vectastain kit) was applied until the color developed. Slides were mounted with Kaiser's glycerol gelatin (Merck). 


\section{Radioactive in situ hybridization}

Radioactive in situ hybridization was performed as described previously (Albrecht et al., 1997; Oster et al., 2003). The Bmal1 probe corresponds to nucleotides 654-1290 (AF015953), the Per2 probe corresponds to nucleotides 229-768 (AF036893), the Dbp probe corresponds to nucleotides 2-951 (NM016974). PCR II Topo or PCR script vectors containing templates were prepared with the Maxi Prep kit (Macherey-Nagel). Plasmids were linearized using the restriction enzymes EcoRI (Per2) and Notl (Bmal1 and Dbp) and purified with the PCR purification kit (Qiagen). $1 \mu \mathrm{g}$ of linearized plasmid was used to generate ${ }^{35}$ S-UTP (Perkin Elmer) labeled RNA probes, using the MAXIscript ${ }^{\circledR}$ In Vitro Transcription Kit (Ambion) and T7 (Bmal1), T3 (Per2) or SP6 (Dbp) polymerases. Transcription setup for ${ }^{35}$ S-UTP labeled antisense RNA probes for clock gene transcripts were performed as described:

- $1 \mu \mathrm{g}$ linearized cDNA template,

- $8 \mu \mathrm{LIEPC}$ water,

○ $2 \mu \mathrm{l} 10 \times$ transcription buffer,

- $1 \mu$ l each rATP, rCTP and rGTP,

$\circ 1 \mu$ RNase inhibitor,

○ $5 \mu \mathrm{l} \alpha^{35} \mathrm{~S}$-UTP $(1 \mathrm{M} / \mu \mathrm{l} ; 1 \mathrm{Ci} / \mathrm{M} ; 19 \mu \mathrm{Ci} / \mu \mathrm{l})$,

○ $1 \mu \mathrm{l}$ RNA polymerase.

The solution was gently mixed and incubated for $2 \mathrm{~h}$ at $37^{\circ} \mathrm{C}$ with agitation (400 rpm). Then the template DNA was degraded:

- $19 \mu \mathrm{L}$ DEPC water,

○ $1.7 \mu \mathrm{l} \mathrm{MgCl} 2(0.3 \mathrm{M})$,

○ $1 \mu \mathrm{I}$ DNase I (2 U).

The solution was gently mixed and incubated for $15 \mathrm{~min}$ at $37^{\circ} \mathrm{C}$ with agitation ( $400 \mathrm{rpm}$ ). The probe was precipitated as followed:

- $100 \mu \mathrm{I}$ DEPC water,

○ $100 \mu$ l yeast tRNA $(1 \mathrm{mg} / \mathrm{ml})$,

○ $250 \mu$ l ammonium acetate (4 M),

○ $1 \mathrm{ml} 100 \%$ ethanol.

Tubes were vortexed and incubated on ice for $10 \mathrm{~min}$ following by centrifugation for $15 \mathrm{~min}$ at $4{ }^{\circ} \mathrm{C}$ (14,000 rpm). The supernatant was carefully removed and the pellet was re-suspended in:

○ $200 \mu \mathrm{L}$ DEPC water, 
○ $200 \mu$ l ammonium acetate (4 M),

○ $800 \mu \mathrm{ll} 100 \%$ ethanol.

Tubes were again vortexed and incubated on ice for $10 \mathrm{~min}$, and then centrifuged for $10 \mathrm{~min}$ at $4{ }^{\circ} \mathrm{C}$ $(14,000 \mathrm{rpm})$ and the supernatant was discarded. The pellets were dissolved in the in situ hybridization buffer (Ambion) and $1 \%$ dithiothreitol (DTT) was added. Incorporation of radioactive nucleotides was determined by liquid scintillation and probes were kept at $-20^{\circ} \mathrm{C}$ overnight.

In situ hybridization was performed on 8- $\mu \mathrm{m}$ paraffin brain sections. Sections were deparaffinized in UltraClear solution (J. T. Baker) and then rehydrated in progressively decreasing concentrations of ethanol (from $100 \%$ to $30 \%$ ). After washing in 1 x PBS, sections were post-fixed for 20 min in $4 \%$ PFA (in PBS, pH 7.4), washed for $5 \mathrm{~min}$ in $1 \times$ PBS, treated with proteinase $\mathrm{K}$ (Roche, $40 \mu \mathrm{g} / \mathrm{ml}$ in 50 $\mathrm{mM}$ Tris/HCl, $5 \mathrm{mM}$ EDTA, $\mathrm{pH}$ 8.5) for $5 \mathrm{~min}$, incubated in $0.2 \mathrm{~N} \mathrm{HCl}$ for $5 \mathrm{~min}$, washed in $1 \times$ PBS for 5 min, re-fixed for $20 \mathrm{~min}$ in $4 \% \mathrm{PFA}$ (in PBS, pH 7.4), acetylated in $0.1 \mathrm{M}$ triethanolamine/ $\mathrm{HCl} \mathrm{pH} 8.0$ (750 $\mu \mathrm{l}$ acetic anhydride per $250 \mathrm{ml}$ solution for $3 \mathrm{~min}$, then another $750 \mu \mathrm{l}$ acetic anhydride for 7 $\mathrm{min}$ ), washed in 1 x PBS for $5 \mathrm{~min}$, incubated in $0.9 \% \mathrm{NaCl}$ for $5 \mathrm{~min}$ and dehydrated in progressively increasing concentrations of ethanol (30\%, $50 \%, 70 \%, 90 \%, 2 \times 100 \%)$ for 30 sec each. Sections were air-dried in an RNase-free chamber. Slides were covered with $100 \mu \mathrm{l}$ of probe diluted in ISH hybridization buffer (Ambion) (+ $0.02 \%$ DTT) and covered with glass cover slips. Hybridization was performed in humidified chambers ( $5 \times$ SSC, $50 \%$ formamide) in an incubation oven at $55^{\circ} \mathrm{C}$ overnight.

The next day slides were washed in removal wash buffer ( 5 x SSC/ 20 mM 2-mercaptoethanol (2-ME)) for $30 \mathrm{~min}$ at $64^{\circ} \mathrm{C}$ in a shaking water bath; after $10 \mathrm{~min}$ cover slips were carefully removed. Formamide washing was performed for $30 \mathrm{~min}$ at $64^{\circ} \mathrm{C}$ ( 2 x SSC / $50 \%$ formamide / $40 \mathrm{mM}$ 2-ME). Sections were incubated in 1 x NTE ( $50 \mathrm{mM} \mathrm{NaCl}, 10 \mathrm{mM}$ Tris-HCl, $5 \mathrm{mM}$ EDTA, pH 8.0) for $15 \mathrm{~min}$ at $37{ }^{\circ} \mathrm{C}$, treated with RNAse for $30 \mathrm{~min}$ at $37^{\circ} \mathrm{C}(20 \mu \mathrm{g} / \mathrm{ml}$ RNAse A in NTE), washed in $1 \times$ NTE for 15 min at $37^{\circ} \mathrm{C}$, incubated in formamide buffer ( $2 \times \mathrm{SSC} / 50 \%$ formamide / $40 \mathrm{mM}$ 2-ME) for $30 \mathrm{~min}$ at $64{ }^{\circ} \mathrm{C}$, washed in $0.1 \times \mathrm{SSC}$ for $15 \mathrm{~min}$ at room temperature and dehydrated in progressively increasing ethanol concentrations ( $30 \%, 60 \%, 80 \%$ ethanol / $0.3 \mathrm{M}$ ammonium acetate, $95 \%, 2 \mathrm{x}$ $100 \%$ ethanol). Slides were air-dried before being exposed to $x$-ray film (Kodak BioMax MS). Developed films were scanned with a densitometer (BioRad) and quantification of relative expression levels was performed by densitometric analysis using the Quantity One software (BioRad). Three sections per brain were analyzed. For background subtraction adjacent hypothalamic areas were used on the same section. 


\section{Western blotting}

For Western blot analyses the following solutions were used. The solutions were filled up with MilliQ water to the denoted volume:

$5 \times$ SDS loading buffer $(\mathrm{pH} \mathrm{7.4,10} \mathrm{ml):}$

○ $2.5 \mathrm{ml} 1 \mathrm{M}$ Tris/HCl, pH 6.8 (250 mM),

○ $0.771 \mathrm{~g}$ DDT,

○ $0.05 \mathrm{~g}$ bromophenol blue,

○ $5 \mathrm{ml} 50 \%$ glycerol with $1 \mathrm{~g}$ SDS.

RIPA buffer:

○ $50 \mathrm{mM}$ Tris-HCl, pH 7.4,

- $150 \mathrm{mM} \mathrm{NaCl}$,

- $1 \%$ Triton $\mathrm{X}-100$,

- $1 \%$ sodium deoxycholate,

○ $0.1 \% \mathrm{SDS}$,

- 1 mM EDTA,

○ protease inhibitors (added freshly).

$10 \times$ electrophoresis buffer $(1 \mathrm{~L})$ :

○ 30.2 g Tris,

- 188 g Glycine,

- $100 \mathrm{ml} 10 \%$ SDS.

$10 \times$ transfer buffer stock $(\mathrm{pH} 8.3,1 \mathrm{~L})$ :

- 29 g glycine,

○ $58 \mathrm{~g}$ Tris,

○ $3.7 \mathrm{~g}$ SDS.

$1 \times$ transfer buffer $(1 \mathrm{~L})$ :

- $100 \mathrm{ml} 10 \times$ transfer buffer,

○ $200 \mathrm{ml}$ methanol,

○ $700 \mathrm{ml}$ MilliQ water,

- $10 \times \operatorname{TBS}(\mathrm{pH} \mathrm{7.4,1} \mathrm{L})$,

- $80 \mathrm{~g} \mathrm{NaCl}$, 
○ $2 \mathrm{~g} \mathrm{KCl}$,

○ $30 \mathrm{~g}$ Tris.

$1 \times \operatorname{TBS}-\mathrm{T}(1 \mathrm{~L})$ :

○ $100 \mathrm{ml} 10 \times \mathrm{TBS}$,

- $1 \mathrm{ml}$ TWEEN 20.

Tissues were homogenized with a pestle in freshly prepared RIPA buffer and sonicated in an ultrasonic bath for $5 \mathrm{~min}$ at $4{ }^{\circ} \mathrm{C}$. Then samples were centrifuged at $13,000 \mathrm{rpm}$ for $20 \mathrm{~min}$ at $4^{\circ} \mathrm{C}$ and the liquid phase was transferred into a new tube. Protein concentration was analyzed by Bradford assay (BioRad) according to the manufacturer's protocol and measured using a spectrophotometer (Eppendorf). Aliquots of protein solution were made and stored at $-80^{\circ} \mathrm{C}$. A $10 \%$ polyacrylamide gel was prepared according to the manufacturer's recommendations (BioRad).

After denaturation at $95^{\circ} \mathrm{C}$ for $8 \mathrm{~min}$, samples were loaded on the gel and run for $30 \mathrm{~min}$ at $50 \mathrm{~V}$ followed by $90 \mathrm{~min}$ at $120 \mathrm{~V}$. Meanwhile the PVDF membrane (Roche) was activated in methanol for $5 \mathrm{~min}$ and then kept in ice cold $1 \times$ transfer buffer. The transfer chamber was assembled according instructions and filled with ice cold $1 \times$ transfer buffer. The gel and the membrane were clamped between sponges and filter paper (Whatman) and subjected to current for $70 \mathrm{~min}$ at $400 \mathrm{~mA}$ at $4{ }^{\circ} \mathrm{C}$. After transfer the membrane was blocked for $60 \mathrm{~min}$ in the blocking solution ( $5 \%$ non-fat dry milk) and the first antibody solution (1:250 in the blocking solution) was applied at $4{ }^{\circ} \mathrm{C}$ overnight. Next day the membrane was incubated with a secondary antibody labeled with horseradish peroxidase (HRP) (1:20000 in the blocking solution) for $1 \mathrm{~h}$ at room temperature. Afterwards the membrane was incubated for $5 \mathrm{~min}$ in PICO chemiluminescent solution (Thermo scientific) and was exposed to the Amersham photographic film (GE Healthcare). Exposed films were developed with the X-OMAT 1000 (Kodak) and analyzed with a densitometer (GS-800 calibrated densitometer, BioRad) and the associated software (QuantityOne).

\section{Molecular biology methods}

\section{Genotyping}

Tail snips were taken from 3-4 week old weaned mice and were lysed overnight at $55^{\circ} \mathrm{C}$ in $400 \mu \mathrm{l}$ tail extraction buffer (200 mM Tris-HCl, pH 8.0, 50 mM EDTA (pH 8.0), 100 mM NaCl, 1 \% SDS) with $10 \mu l$ proteinase $\mathrm{K}(10 \mathrm{mg} / \mathrm{ml})$. Afterwards tissue debris was spun down at 13,000 rpm for $10 \mathrm{~min}$ and 200 $\mu$ I DNA-containing supernatant was mixed with $400 \mu \mathrm{l}$ of ice-cold $100 \%$ ethanol. Precipitated DNA was washed with $200 \mu \mathrm{l}$ of $70 \%$ ethanol and air-dried for $10 \mathrm{~min}$. DNA was dissolved in Tris-EDTA (10 $\mathrm{mM}$ Tris, $\mathrm{pH}$ 8.0, $1 \mathrm{mM}$ EDTA, pH 8.0). Genotyping PCR was carried out with a FlexCycler PCR machine (Analytik Jena). 
Per2::Luc genotyping:

Setup:

- $0.5 \mu \mathrm{l}$ DNA (approx. $200 \mathrm{ng} / \mu \mathrm{l}$ ),

- $\mu l$ ammonium buffer,

- $\mu l d N T P$,

- $\mu l$ forward primer $(10 \mu \mathrm{M})$,

- $\mu l$ reverse primer $1(10 \mu \mathrm{M})$,

- $\mu \mathrm{l}$ reverse primer $2(10 \mu \mathrm{M})$,

- $\mu l$ Amplicon Taq polymerase,

- $7.8 \mu \mathrm{l}$ PCR-graded water.

Total volume: $10 \mu$ l.

Cycling program:

- $3 \min 95^{\circ} \mathrm{C}$,

- $30 \sec 94^{\circ} \mathrm{C}$,

- $30 \sec 65^{\circ} \mathrm{C}, 36 \mathrm{x}$,

- $1 \min 72^{\circ} \mathrm{C}$,

- $7 \min 72^{\circ} \mathrm{C}$,

- keep at $4{ }^{\circ} \mathrm{C}$.

DNA length of the wild-type allele is approx. 200 bp, Per2::Luc allele-approx. 720 bp.

Bmal1flox genotyping:

Setup:

- $1 \mu$ I DNA (approx. $200 \mathrm{ng} / \mu \mathrm{l}$ ),

- $2.5 \mu$ l standard PCR buffer,

- $1 \mu \mathrm{ldNTPs}$,

- $1 \mu \mathrm{l} \mathrm{MgCl}$,

- $1 \mu \mathrm{l}$ forward primer $1(10 \mu \mathrm{M})$,

- $1 \mu \mathrm{l}$ forward primer $2(10 \mu \mathrm{M})$,

- $2 \mu$ reverse primer $(10 \mu \mathrm{M})$,

- $0.5 \mu$ Amplicon Taq polymerase,

- $16 \mu$ l PCR-graded water.

Total volume: $25 \mu \mathrm{l}$.

Cycling program:

- $3 \min 94^{\circ} \mathrm{C}$,

- $30 \sec 94^{\circ} \mathrm{C}$,

- $1 \min 59^{\circ} \mathrm{C}, 35 \mathrm{x}$,

- $1 \min 72^{\circ} \mathrm{C}$,

- $5 \min 72^{\circ} \mathrm{C}$,

- keep at $4{ }^{\circ} \mathrm{C}$.

Approximate DNA lengths of the amplified alleles are: wild-type-420 bp, floxed-524 bp, knockout -726 bp.

Cre genotyping: 
Setup:

- $1 \mu$ l DNA (approx. $200 \mathrm{ng} / \mu \mathrm{l}$ ),

- $2.0 \mu \mathrm{l}$ standard PCR buffer,

- $0.4 \mu \mathrm{ldNTPs}$,

- $1.2 \mu$ forward primer $(10 \mu \mathrm{M})$,

- $1.2 \mu$ reverse primer $(10 \mu \mathrm{M})$,

- $0.15 \mu \mathrm{l}$ Amplicon Taq polymerase,

- $14.05 \mu$ I PCR-graded water.

Total volume: $20 \mu \mathrm{l}$.

Cycling program:

- $4 \min 94^{\circ} \mathrm{C}$,

- $30 \sec 94^{\circ} \mathrm{C}$,

- $30 \sec 62^{\circ} \mathrm{C}, 30 \mathrm{x}$,

- $30 \sec 72^{\circ} \mathrm{C}$,

- $5: 00 \min 72{ }^{\circ} \mathrm{C}$,

- keep at $4{ }^{\circ} \mathrm{C}$.

The amplified DNA is approx. $260 \mathrm{bp}$.

Clock ${ }^{\Delta 19}$ genotyping:

Setup:

- $1 \mu$ I DNA (approx. $200 \mathrm{ng} / \mu \mathrm{l}$ ),

- $10 \mu \mathrm{l}$ QIAGEN HotStarTaq master mix,

- $\mu l$ forward primer $(10 \mu \mathrm{M})$,

- $\mu$ r reverse primer $(10 \mu \mathrm{M})$,

- $7.0 \mu \mathrm{l}$ PCR-graded water.

Total volume: $20 \mu \mathrm{l}$.

Cycling program:

- $15 \min 95^{\circ} \mathrm{C}$,

- $1 \min 94^{\circ} \mathrm{C}$,

- $1 \min 68^{\circ} \mathrm{C}, 35 \mathrm{x}$,

- $1 \min 72^{\circ} \mathrm{C}$,

- $10 \min 72^{\circ} \mathrm{C}$,

- keep at $4{ }^{\circ} \mathrm{C}$.

After amplification $1 \mu \mathrm{L}$ Hincll (10,000 U/mL, R0103S, NEB) was added to the reaction, mixed and incubated at $37^{\circ} \mathrm{C}$ overnight. The digested PCR product was detected on 1.0-1.4 \% agarose gels.

The DNA length of the Clock mutant allele is $460 \mathrm{bp}$ (no Hincll digestion), of the wild-type allele398 bp (after cleavage by Hincll).

\begin{tabular}{|l|l|}
\hline Genotyping primer & Sequence \\
\hline Per2::Luc, forward & CTGTGTTTACTGCGAGAGT \\
\hline Per2::Luc, reverse 1 & GGGTCCATGTGATTAGAAAC \\
\hline
\end{tabular}




\begin{tabular}{|l|l|}
\hline Per2::Luc, reverse 2 & TAAAACCGGGAGGTAGATGAGA \\
\hline Bmal1flox, forward 1 & GGAGGGTGAGAAAACAGAGGCAGG \\
\hline Bmal1flox, forward 2 & GCAGAGCCTCACATCTGACAGGAG \\
\hline Bmal1 flox, reverse & GATTAAAGGCGTGTGCCACCACACC \\
\hline Cre, forward & CGATGCAACGAGTGATGAGGTTCG \\
\hline Cre, reverse & AGCATTGCTGTCACTTGGTCGTGG \\
\hline Clock $^{119}$ Hinc, forward & $\begin{array}{l}\text { GCAAGAAGAACTAAGGAAATTCAAGAGCAACTTCAGATGGTCCATGGTCA } \\
\text { AGGGCTACAGTT }\end{array}$ \\
\hline Clock $^{\Delta 19}$ Hinc, reverse & TAGTGCCCTAGATGGCCCTGTTGG \\
\hline
\end{tabular}

\section{RNA isolation}

RNA was isolated from frozen $\left(-80^{\circ} \mathrm{C}\right)$ tissues with TRIzol (Invitrogen) according to the manufacturer's protocol. Isolated RNA was dissolved in double distilled water and stored at $-80^{\circ} \mathrm{C}$. The concentration was determined with the ND-1000 NanoDrop spectrophotometer (Peqlab). The RNA integrity was verified by $1.5 \%$ agarose gel electrophoresis.

\section{cDNA synthesis}

cDNA was synthesized from RNA samples with the MulitScribe Reverse Trancription Kit including RNase inhibitor (Applied Biosystems) according to the manufacturer's protocol using 2-3 $\mu \mathrm{g}$ of total RNA.

\section{Quantitative real-time PCR (qPCR)}

qPCR was performed using the CFX96 Real-Time detection system (BioRad) and iQ-SYBR Green Supermix (BioRad) or GoTaq qPCR Master Mix (Promega).

Setup:

- $5 \mu \mathrm{l}$ cDNA (1:20 dilution),

- $5 \mu$ primer mix (1 $\mu \mathrm{M}$ of each primer),

- $10 \mu \mathrm{l} 2 \mathrm{x}$ qPCR mix.

Total volume: $20 \mu$ l.

Cycling program:

- $7 \min 95^{\circ} \mathrm{C}$,

- $10 \sec 95^{\circ} \mathrm{C}$,

- $25 \sec 60^{\circ} \mathrm{C}, 40 \mathrm{x}$,

- $20 \sec 72{ }^{\circ} \mathrm{C}$,

- $5 \sec 65-95^{\circ} \mathrm{C}+0.5^{\circ} \mathrm{C}$ per cycle.

Data analysis was performed by calculation of $d C(t)$ (threshold cycling numbers) values for each curve using the CFX96 software. Quantification of expression levels was performed by the $\Delta \Delta C(t)$ method relative to EF1a or Hprt as housekeeping reference genes according to the formula:

$$
\text { Ratio }=2^{- \text {(CTtarget }- \text { CTref })} \text {. }
$$


Statistical analyses were done with GraphPad Prism software (GraphPad Software). Circadian profiles of clock gene expression were normalized to the maximum value of the respective wild-type dataset.

Sequences of qPCR primers:

\begin{tabular}{|c|c|}
\hline$L p \mid$ & $\begin{array}{l}\text { Forward primer 5'-GGACGGTAACGGGAATGTATG-3' } \\
\text { Reverse primer 5'-ACGTTGTCTAGGGGGTACTTAAA -3' }\end{array}$ \\
\hline Fatp1 (SIc27a1) & $\begin{array}{l}\text { Forward primer 5'-CGCTTTCTGCGTATCGTCTG-3' } \\
\text { Reverse primer 5'-GATGCACGGGATCGTGTCT-3' }\end{array}$ \\
\hline Fatp4 (SIc27a4) & $\begin{array}{l}\text { Forward primer 5'-TGAGATGGCCTCAGCTATCTG-3' } \\
\text { Reverse primer 5'-TGCCCGATGTGTAGATGTAGAA-3' }\end{array}$ \\
\hline Cd36 & $\begin{array}{l}\text { Forward primer 5'-CCGAGGACCACACTGTGTC-3' } \\
\text { Reverse primer 5'-AACCCCACAAGAGTTCTTTCAAA-3' }\end{array}$ \\
\hline Acs/1 & $\begin{array}{l}\text { Forward primer 5'-ACCACCTTCTGGTATGCCAC-3' } \\
\text { Reverse primer 5'-TGACATCGTCGTAGTAGTACACC-3' }\end{array}$ \\
\hline Gpam & $\begin{array}{l}\text { Forward primer 5'-ACGCACACAAGGCACAGAG-3' } \\
\text { Reverse primer 5'-TGCTGCTCAGTACATTCTCAGTA-3' }\end{array}$ \\
\hline Agpat2 & $\begin{array}{l}\text { Forward primer 5'-CTGGTTCGTTCGGTCCTTCAA-3' } \\
\text { Reverse primer 5'-CTTGGCGATCTGCACACAG-3' }\end{array}$ \\
\hline Dgat1 & $\begin{array}{l}\text { Forward primer 5'-TCCGTCCAGGGTGGTAGTG-3' } \\
\text { Reverse primer 5'-TGAACAAAGAATCTTGCAGACGA-3' }\end{array}$ \\
\hline Dgat2 & $\begin{array}{l}\text { Forward primer 5'-TTCCTGGCATAAGGCCCTATT-3' } \\
\text { Reverse primer 5'-AGTCTATGGTGTCTCGGTTGAC-3' }\end{array}$ \\
\hline Plin1 & $\begin{array}{l}\text { Forward primer 5'-AGATCCCGGCTCTTCAATACC-3' } \\
\text { Reverse primer 5'-AGAACCTTGTCAGAGGTGCTT-3' }\end{array}$ \\
\hline Atgl & $\begin{array}{l}\text { Forward primer 5'-CAACGCCACTCACATCTACGG-3' } \\
\text { Reverse primer 5'-TCACCAGGTTGAAGGAGGGAT-3' }\end{array}$ \\
\hline $\mathrm{Hsl}$ & $\begin{array}{l}\text { Forward primer 5'-GGCTCACAGTTACCATCTCACC-3' } \\
\text { Reverse primer 5'-GAGTACCTTGCTGTCCTGTCC-3' }\end{array}$ \\
\hline Mgll & $\begin{array}{l}\text { Forward primer 5'-CGGACTTCCAAGTTTTTGTCAGA-3' } \\
\text { Reverse primer 5'-GCAGCCACTAGGATGGAGATG-3' }\end{array}$ \\
\hline Lpin1 & $\begin{array}{l}\text { Forward primer 5'-CTCCGCTCCCGAGAGAAAG-3' } \\
\text { Reverse primer 5'-TCATGTGCAAATCCACGGACT-3' }\end{array}$ \\
\hline Got2 & $\begin{array}{l}\text { Forward primer 5'-CCTGGGCGAGAACAATGAAGT-3' } \\
\text { Reverse primer 5'-ATGGGCGTGTGATTTCCCC-3' }\end{array}$ \\
\hline Cav1 & $\begin{array}{l}\text { Forward primer 5'-GCGACCCCAAGCATCTCAA-3' } \\
\text { Reverse primer 5'-ATGCCGTCGAAACTGTGTGT-3' }\end{array}$ \\
\hline Cav2 & $\begin{array}{l}\text { Forward primer 5'-TCACCAGCTCAACTCTCATCT-3' } \\
\text { Reverse primer 5'-GCCAGAAATACGGTCAGGAACT-3' }\end{array}$ \\
\hline Fitm2 & $\begin{array}{l}\text { Forward primer 5'-TCGGTCGTCAAGGAGCTGT-3' } \\
\text { Reverse primer 5'-CAAAATACACGTTGAGGACGTTG-3' }\end{array}$ \\
\hline Nr1h2 & $\begin{array}{l}\text { Forward primer 5'-GCCTGGGAATGGTTCTCCTC-3' } \\
\text { Reverse primer 5'-AGATGACCACGATGTAGGCAG-3' }\end{array}$ \\
\hline Nr1h3 & $\begin{array}{l}\text { Forward primer 5'-GTCAACTGGGGTTGCTTTAGG-3' } \\
\text { Reverse primer 5'-GACGAAGCTCTGTCGGCTC-3' }\end{array}$ \\
\hline Acs/4 & $\begin{array}{l}\text { Forward primer 5'-CCTGAGGGGCTTGAAATTCAC-3' } \\
\text { Reverse primer 5'-GTTGGTCTACTTGGAGGAACG-3' }\end{array}$ \\
\hline Acs/5 & $\begin{array}{l}\text { Forward primer 5'- AACCAGTCTGTGGGGATTGAG -3' } \\
\text { Reverse primer 5'- CGTCTTGGCGTCTGAGAAGTA -3' }\end{array}$ \\
\hline
\end{tabular}




\begin{tabular}{|l|l|}
\hline Agpat6 & $\begin{array}{l}\text { Forward primer 5'-AACCTCCTGGGTATCTCCCTG-3' } \\
\text { Reverse primer 5'-CCGTTGGTGTAGGGCTTGT-3' }\end{array}$ \\
\hline Agpat9 & $\begin{array}{l}\text { Forward primer 5'-CGGATTATCCCTGGGTATCTCG-3' } \\
\text { Reverse primer 5'-CGAAGTCCCTTCCTCGAAGAC-3' }\end{array}$ \\
\hline Mogat1 & $\begin{array}{l}\text { Forward primer 5'-CTCGTGCAGGTGTGCATTG-3' } \\
\text { Reverse primer 5'-GCGTTTTGACAAGACAGATTGG-3' }\end{array}$ \\
\hline Pnpla3 & $\begin{array}{l}\text { Forward primer 5'-TCACCTTCGTGTGCAGTCTC-3' } \\
\text { Reverse primer 5'-CCTGGAGCCCGTCTCTGAT-3' }\end{array}$ \\
\hline Abhd5 & $\begin{array}{l}\text { Forward primer 5'-TGGTGTCCCACATCTACATCA-3' } \\
\text { Reverse primer 5'-CAGCGTCCATATTCTGTTTCCA-3' }\end{array}$ \\
\hline Fabp4 & $\begin{array}{l}\text { Forward primer 5'-AAGGTGAAGAGCATCATAACCCT-3' } \\
\text { Reverse primer 5'-TCACGCCTTTCATAACACATTCC-3' }\end{array}$ \\
\hline Leptin & $\begin{array}{l}\text { Forward primer 5'-TGAAAGAGCTAGGAGTAGGACTG-3' } \\
\text { Reverse primer 5'-CTCTCGGTTTTGACCGTGATG-3' }\end{array}$ \\
\hline Ef1a & $\begin{array}{l}\text { Forward primer 5'-GAGACCCCTGTGTCGGTTC -3' } \\
\text { Reverse primer 5'-CTGCGTGTGTGAAATGTCATTG -3' }\end{array}$ \\
\hline Hprt & $\begin{array}{l}\text { Forward primer 5'-CACATCCCAGGCTGACTGT-3' } \\
\text { Reverse primer 5'-TCGGTGGAATCCATTTTGTT-3' }\end{array}$ \\
\hline Bmal1 & $\begin{array}{l}\text { Forward primer 5'-GTTGGGCTTACCTCACTGCT-3' } \\
\text { Reverse primer 5'-TGATGGCCTCCCATCTCCTT-3' }\end{array}$ \\
\hline Per2 & $\begin{array}{l}\text { Forward primer 5'-ATCAGCGACTTCATGTCTCC-3' } \\
\text { Reverse primer 5'-CTCCCTTGCATTCTTGATCC-3' }\end{array}$ \\
\hline Dbp & $\begin{array}{l}\text { Forward primer 5'-GCCAAGTTTGTGGAGTTCCTG-3' } \\
\text { Reverse primer 5'-CTTGCACCTTGACCAGGTAGG-3' }\end{array}$ \\
\hline & $\begin{array}{l}\text { Forward primer 5'-AATGACCTTTGAACCTGATCCCGCT-3' } \\
\text { Reverse primer 5'-GCTCCAGTACTTCTCATCCTTCTGT-3' }\end{array}$ \\
\hline
\end{tabular}

\section{Statistical Analyses}

All results are expressed as a mean \pm S.E.M. For statistical comparison unpaired two-tailed Student's t-tests and 1-way or 2-way ANOVAs with Bonferroni post-tests were performed using Prism 5 software (GraphPad Software). P-values $<0.05$ were considered significant. 


\section{References}

Acheson, K.J., Gremaud, G., Meirim, I., Montigon, F., Krebs, Y., Fay, L.B., Gay, L.J., Schneiter, P., Schindler, C., and Tappy, L. (2004). Metabolic effects of caffeine in humans: lipid oxidation or futile cycling? Am J Clin Nutr 79, 40-46.

Ahima, R.S., Prabakaran, D., and Flier, J.S. (1998). Postnatal leptin surge and regulation of circadian rhythm of leptin by feeding. Implications for energy homeostasis and neuroendocrine function. J Clin Invest 101, 1020-1027.

Ahmadian, M., Abbott, M.J., Tang, T., Hudak, C.S., Kim, Y., Bruss, M., Hellerstein, M.K., Lee, H.Y., Samuel, V.T., Shulman, G.I., et al. (2011). Desnutrin/ATGL is regulated by AMPK and is required for a brown adipose phenotype. Cell Metab 13, 739-748.

Akiyama, M., Sakai, K., Ogawa, M., McMillan, J.R., Sawamura, D., and Shimizu, H. (2007). Novel duplication mutation in the patatin domain of adipose triglyceride lipase (PNPLA2) in neutral lipid storage disease with severe myopathy. Muscle Nerve 36, 856-859.

Albrecht, U. (2012). Timing to perfection: the biology of central and peripheral circadian clocks. Neuron 74, 246-260.

Albrecht, U., Sun, Z.S., Eichele, G., and Lee, C.C. (1997). A differential response of two putative mammalian circadian regulators, mper1 and mper2, to light. Cell 91, 1055-1064.

Ando, H., Oshima, Y., Yanagihara, H., Hayashi, Y., Takamura, T., Kaneko, S., and Fujimura, A. (2006). Profile of rhythmic gene expression in the livers of obese diabetic KK-A(y) mice. Biochem Biophys Res Commun 346, 1297-1302.

Ando, H., Yanagihara, H., Hayashi, Y., Obi, Y., Tsuruoka, S., Takamura, T., Kaneko, S., and Fujimura, A. (2005). Rhythmic messenger ribonucleic acid expression of clock genes and adipocytokines in mouse visceral adipose tissue. Endocrinology 146, 5631-5636.

Arble, D.M., Bass, J., Laposky, A.D., Vitaterna, M.H., and Turek, F.W. (2009). Circadian timing of food intake contributes to weight gain. Obesity (Silver Spring) 17, 2100-2102.

Arita, Y., Kihara, S., Ouchi, N., Takahashi, M., Maeda, K., Miyagawa, J., Hotta, K., Shimomura, I., Nakamura, T., Miyaoka, K., et al. (1999). Paradoxical decrease of an adipose-specific protein, adiponectin, in obesity. Biochem Biophys Res Commun 257, 79-83.

Aschoff, J. (1965). Circadian Rhythms in Man. Science 148, 1427-1432.

Aschoff, J., and Tokura, H. (1986). Circadian activity rhythms in squirrel monkeys: entrainment by temperature cycles. J Biol Rhythms 1, 91-99. 
Asher, G., Gatfield, D., Stratmann, M., Reinke, H., Dibner, C., Kreppel, F., Mostoslavsky, R., Alt, F.W., and Schibler, U. (2008). SIRT1 regulates circadian clock gene expression through PER2 deacetylation. Cell 134, 317-328.

Balsalobre, A., Brown, S.A., Marcacci, L., Tronche, F., Kellendonk, C., Reichardt, H.M., Schutz, G., and Schibler, U. (2000). Resetting of circadian time in peripheral tissues by glucocorticoid signaling. Science 289, 2344-2347.

Balsalobre, A., Damiola, F., and Schibler, U. (1998). A serum shock induces circadian gene expression in mammalian tissue culture cells. Cell 93, 929-937.

Bass, J., and Takahashi, J.S. (2010). Circadian integration of metabolism and energetics. Science $330,1349-1354$.

Benedict, C., Shostak, A., Lange, T., Brooks, S.J., Schioth, H.B., Schultes, B., Born, J., Oster, H., and Hallschmid, M. (2012). Diurnal rhythm of circulating nicotinamide phosphoribosyltransferase (Nampt/visfatin/PBEF): impact of sleep loss and relation to glucose metabolism. J Clin Endocrinol Metab 97, E218-222.

Berson, D.M., Dunn, F.A., and Takao, M. (2002). Phototransduction by retinal ganglion cells that set the circadian clock. Science 295, 1070-1073.

Blakemore, A.I., Meyre, D., Delplanque, J., Vatin, V., Lecoeur, C., Marre, M., Tichet, J., Balkau, B., Froguel, P., and Walley, A.J. (2009). A rare variant in the visfatin gene (NAMPT/PBEF1) is associated with protection from obesity. Obesity (Silver Spring) 17, 1549-1553.

Borglum, J.D., Vassaux, G., Richelsen, B., Gaillard, D., Darimont, C., Ailhaud, G., and Negrel, R. (1996). Changes in adenosine A1- and A2-receptor expression during adipose cell differentiation. Mol Cell Endocrinol 117, 17-25.

Brown, S.A., Zumbrunn, G., Fleury-Olela, F., Preitner, N., and Schibler, U. (2002). Rhythms of mammalian body temperature can sustain peripheral circadian clocks. Curr Biol 12, 1574-1583.

Bunger, M.K., Wilsbacher, L.D., Moran, S.M., Clendenin, C., Radcliffe, L.A., Hogenesch, J.B., Simon, M.C., Takahashi, J.S., and Bradfield, C.A. (2000). Mop3 is an essential component of the master circadian pacemaker in mammals. Cell 103, 1009-1017.

Bur, I.M., Cohen-Solal, A.M., Carmignac, D., Abecassis, P.Y., Chauvet, N., Martin, A.O., van der Horst, G.T., Robinson, I.C., Maurel, P., Mollard, P., et al. (2009). The circadian clock components CRY1 and CRY2 are necessary to sustain sex dimorphism in mouse liver metabolism. J Biol Chem 284, 90669073.

Chandran, M., Phillips, S.A., Ciaraldi, T., and Henry, R.R. (2003). Adiponectin: more than just another fat cell hormone? Diabetes Care 26, 2442-2450. 
Chen, M.P., Chung, F.M., Chang, D.M., Tsai, J.C., Huang, H.F., Shin, S.J., and Lee, Y.J. (2006). Elevated plasma level of visfatin/pre-B cell colony-enhancing factor in patients with type 2 diabetes mellitus. J Clin Endocrinol Metab 91, 295-299.

Cheng, M.Y., Bullock, C.M., Li, C., Lee, A.G., Bermak, J.C., Belluzzi, J., Weaver, D.R., Leslie, F.M., and Zhou, Q.Y. (2002). Prokineticin 2 transmits the behavioural circadian rhythm of the suprachiasmatic nucleus. Nature 417, 405-410.

Cho, H., Zhao, X., Hatori, M., Yu, R.T., Barish, G.D., Lam, M.T., Chong, L.W., DiTacchio, L., Atkins, A.R., Glass, C.K., et al. (2012). Regulation of circadian behaviour and metabolism by REV-ERB-alpha and REV-ERB-beta. Nature 485, 123-127.

Cook, K.S., Min, H.Y., Johnson, D., Chaplinsky, R.J., Flier, J.S., Hunt, C.R., and Spiegelman, B.M. (1987). Adipsin: a circulating serine protease homolog secreted by adipose tissue and sciatic nerve. Science 237, 402-405.

Cretenet, G., Le Clech, M., and Gachon, F. (2010). Circadian clock-coordinated $12 \mathrm{Hr}$ period rhythmic activation of the IRE1alpha pathway controls lipid metabolism in mouse liver. Cell Metab $11,47-57$.

Dallmann, R., Viola, A.U., Tarokh, L., Cajochen, C., and Brown, S.A. (2012). The human circadian metabolome. Proc Natl Acad Sci U S A 109, 2625-2629.

Dallmann, R., and Weaver, D.R. (2010). Altered body mass regulation in male mPeriod mutant mice on high-fat diet. Chronobiol Int 27, 1317-1328.

Damiola, F., Le Minh, N., Preitner, N., Kornmann, B., Fleury-Olela, F., and Schibler, U. (2000). Restricted feeding uncouples circadian oscillators in peripheral tissues from the central pacemaker in the suprachiasmatic nucleus. Genes Dev 14, 2950-2961.

De Bacquer, D., Van Risseghem, M., Clays, E., Kittel, F., De Backer, G., and Braeckman, L. (2009). Rotating shift work and the metabolic syndrome: a prospective study. Int J Epidemiol 38, 848854.

De Boer, S.F., and Van der Gugten, J. (1987). Daily variations in plasma noradrenaline, adrenaline and corticosterone concentrations in rats. Physiol Behav 40, 323-328.

Debruyne, J.P., Noton, E., Lambert, C.M., Maywood, E.S., Weaver, D.R., and Reppert, S.M. (2006). A clock shock: mouse CLOCK is not required for circadian oscillator function. Neuron 50, 465477.

Delezie, J., Dumont, S., Dardente, H., Oudart, H., Grechez-Cassiau, A., Klosen, P., Teboul, M., Delaunay, F., Pevet, P., and Challet, E. (2012). The nuclear receptor REV-ERBalpha is required for the daily balance of carbohydrate and lipid metabolism. FASEB J 26, 3321-3335. 
Dibner, C., Sage, D., Unser, M., Bauer, C., d'Eysmond, T., Naef, F., and Schibler, U. (2009). Circadian gene expression is resilient to large fluctuations in overall transcription rates. EMBO J 28, 123-134.

Dibner, C., Schibler, U., and Albrecht, U. (2010). The mammalian circadian timing system: organization and coordination of central and peripheral clocks. Annu Rev Physiol 72, 517-549.

Doi, M., Takahashi, Y., Komatsu, R., Yamazaki, F., Yamada, H., Haraguchi, S., Emoto, N., Okuno, Y., Tsujimoto, G., Kanematsu, A., et al. (2010). Salt-sensitive hypertension in circadian clock-deficient Cry-null mice involves dysregulated adrenal Hsd3b6. Nat Med 16, 67-74.

Duncan, R.E., Ahmadian, M., Jaworski, K., Sarkadi-Nagy, E., and Sul, H.S. (2007). Regulation of lipolysis in adipocytes. Annu Rev Nutr 27, 79-101.

Dushay, M.S., Konopka, R.J., Orr, D., Greenacre, M.L., Kyriacou, C.P., Rosbash, M., and Hall, J.C. (1990). Phenotypic and genetic analysis of Clock, a new circadian rhythm mutant in Drosophila melanogaster. Genetics 125, 557-578.

Dutt, A.K., and Muller, S.C. (1993). Effect of Stirring and Temperature on the BelousovZhabotinskii Reaction in a Cstr. J Phys Chem-Us 97, 10059-10063.

Eckel-Mahan, K.L., Patel, V.R., Mohney, R.P., Vignola, K.S., Baldi, P., and Sassone-Corsi, P. (2012). Coordination of the transcriptome and metabolome by the circadian clock. Proc Natl Acad Sci U S A 109, 5541-5546.

Edgar, R.S., Green, E.W., Zhao, Y., van Ooijen, G., Olmedo, M., Qin, X., Xu, Y., Pan, M., Valekunja, U.K., Feeney, K.A., et al. (2012). Peroxiredoxins are conserved markers of circadian rhythms. Nature 485, 459-464.

Eguchi, J., Wang, X., Yu, S., Kershaw, E.E., Chiu, P.C., Dushay, J., Estall, J.L., Klein, U., MaratosFlier, E., and Rosen, E.D. (2011). Transcriptional control of adipose lipid handling by IRF4. Cell Metab 13, 249-259.

Ellingsen, T., Bener, A., and Gehani, A.A. (2007). Study of shift work and risk of coronary events. J R Soc Promot Health 127, 265-267.

Ellis, J.M., Li, L.O., Wu, P.C., Koves, T.R., Ilkayeva, O., Stevens, R.D., Watkins, S.M., Muoio, D.M., and Coleman, R.A. (2010). Adipose acyl-CoA synthetase-1 directs fatty acids toward beta-oxidation and is required for cold thermogenesis. Cell Metab 12, 53-64.

Englund, A., Kovanen, L., Saarikoski, S.T., Haukka, J., Reunanen, A., Aromaa, A., Lonnqvist, J., and Partonen, T. (2009). NPAS2 and PER2 are linked to risk factors of the metabolic syndrome. J Circadian Rhythms 7, 5.

Fischer, J., Lefevre, C., Morava, E., Mussini, J.M., Laforet, P., Negre-Salvayre, A., Lathrop, M., and Salvayre, R. (2007). The gene encoding adipose triglyceride lipase (PNPLA2) is mutated in neutral lipid storage disease with myopathy. Nat Genet 39, 28-30. 
Fonken, L.K., Workman, J.L., Walton, J.C., Weil, Z.M., Morris, J.S., Haim, A., and Nelson, R.J. (2010). Light at night increases body mass by shifting the time of food intake. Proc Natl Acad Sci U S A $107,18664-18669$.

Francis, A.J., and Coleman, G.J. (1997). Phase response curves to ambient temperature pulses in rats. Physiol Behav 62, 1211-1217.

Friedman, J.M., and Halaas, J.L. (1998). Leptin and the regulation of body weight in mammals. Nature 395, 763-770.

Froy, O. (2010). Metabolism and circadian rhythms--implications for obesity. Endocr Rev 31, 124.

Fukuda, N., and Ontko, J.A. (1984). Interactions between fatty acid synthesis, oxidation, and esterification in the production of triglyceride-rich lipoproteins by the liver. J Lipid Res 25, 831-842.

Gachon, F., Olela, F.F., Schaad, O., Descombes, P., and Schibler, U. (2006). The circadian PARdomain basic leucine zipper transcription factors DBP, TEF, and HLF modulate basal and inducible xenobiotic detoxification. Cell Metab 4, 25-36.

Gavrila, A., Peng, C.K., Chan, J.L., Mietus, J.E., Goldberger, A.L., and Mantzoros, C.S. (2003). Diurnal and ultradian dynamics of serum adiponectin in healthy men: comparison with leptin, circulating soluble leptin receptor, and cortisol patterns. J Clin Endocrinol Metab 88, 2838-2843.

Gekakis, N., Staknis, D., Nguyen, H.B., Davis, F.C., Wilsbacher, L.D., King, D.P., Takahashi, J.S., and Weitz, C.J. (1998). Role of the CLOCK protein in the mammalian circadian mechanism. Science 280, 1564-1569.

Gibbs, J.E., Blaikley, J., Beesley, S., Matthews, L., Simpson, K.D., Boyce, S.H., Farrow, S.N., Else, K.J., Singh, D., Ray, D.W., et al. (2012). The nuclear receptor REV-ERBalpha mediates circadian regulation of innate immunity through selective regulation of inflammatory cytokines. Proc Natl Acad Sci U S A 109, 582-587.

Golombek, D.A., and Rosenstein, R.E. (2010). Physiology of circadian entrainment. Physiol Rev 90, 1063-1102.

Gottlieb, D.J., Punjabi, N.M., Newman, A.B., Resnick, H.E., Redline, S., Baldwin, C.M., and Nieto, F.J. (2005). Association of sleep time with diabetes mellitus and impaired glucose tolerance. Arch Intern Med 165, 863-867.

Green, C.B., Takahashi, J.S., and Bass, J. (2008). The meter of metabolism. Cell 134, 728-742.

Green, R.M., Tingay, S., Wang, Z.Y., and Tobin, E.M. (2002). Circadian rhythms confer a higher level of fitness to Arabidopsis plants. Plant Physiol 129, 576-584.

Grimaldi, B., Bellet, M.M., Katada, S., Astarita, G., Hirayama, J., Amin, R.H., Granneman, J.G., Piomelli, D., Leff, T., and Sassone-Corsi, P. (2010). PER2 controls lipid metabolism by direct regulation of PPARgamma. Cell Metab 12, 509-520. 
Guillaumond, F., Grechez-Cassiau, A., Subramaniam, M., Brangolo, S., Peteri-Brunback, B., Staels, B., Fievet, C., Spelsberg, T.C., Delaunay, F., and Teboul, M. (2010). Kruppel-like factor KLF10 is a link between the circadian clock and metabolism in liver. Mol Cell Biol 30, 3059-3070.

Guler, A.D., Ecker, J.L., Lall, G.S., Haq, S., Altimus, C.M., Liao, H.W., Barnard, A.R., Cahill, H., Badea, T.C., Zhao, H., et al. (2008). Melanopsin cells are the principal conduits for rod-cone input to non-image-forming vision. Nature 453, 102-105.

Gumz, M.L., Stow, L.R., Lynch, I.J., Greenlee, M.M., Rudin, A., Cain, B.D., Weaver, D.R., and Wingo, C.S. (2009). The circadian clock protein Period 1 regulates expression of the renal epithelial sodium channel in mice. J Clin Invest 119, 2423-2434.

Guo, B., Chatterjee, S., Li, L., Kim, J.M., Lee, J., Yechoor, V.K., Minze, L.J., Hsueh, W., and Ma, K. (2012). The clock gene, brain and muscle Arnt-like 1, regulates adipogenesis via Wnt signaling pathway. FASEB J 26, 3453-3463.

Haemmerle, G., Lass, A., Zimmermann, R., Gorkiewicz, G., Meyer, C., Rozman, J., Heldmaier, G., Maier, R., Theussl, C., Eder, S., et al. (2006). Defective lipolysis and altered energy metabolism in mice lacking adipose triglyceride lipase. Science 312, 734-737.

Haemmerle, G., Zimmermann, R., Hayn, M., Theussl, C., Waeg, G., Wagner, E., Sattler, W., Magin, T.M., Wagner, E.F., and Zechner, R. (2002). Hormone-sensitive lipase deficiency in mice causes diglyceride accumulation in adipose tissue, muscle, and testis. J Biol Chem 277, 4806-4815.

Haider, D.G., Schindler, K., Schaller, G., Prager, G., Wolzt, M., and Ludvik, B. (2006). Increased plasma visfatin concentrations in morbidly obese subjects are reduced after gastric banding. J Clin Endocrinol Metab 91, 1578-1581.

Hardin, P.E., Hall, J.C., and Rosbash, M. (1990). Feedback of the Drosophila period gene product on circadian cycling of its messenger RNA levels. Nature 343, 536-540.

Harmer, S.L., Hogenesch, J.B., Straume, M., Chang, H.S., Han, B., Zhu, T., Wang, X., Kreps, J.A., and Kay, S.A. (2000). Orchestrated transcription of key pathways in Arabidopsis by the circadian clock. Science 290, 2110-2113.

Harmer, S.L., Panda, S., and Kay, S.A. (2001). Molecular bases of circadian rhythms. Annu Rev Cell Dev Biol 17, 215-253.

Hasselbalch, S.G., Knudsen, G.M., Jakobsen, J., Hageman, L.P., Holm, S., and Paulson, O.B. (1994). Brain metabolism during short-term starvation in humans. J Cereb Blood Flow Metab 14, 125131.

Hastings, J.W., and Sweeney, B.M. (1957). On the Mechanism of Temperature Independence in a Biological Clock. Proc Natl Acad Sci U S A 43, 804-811. 
Hatori, M., Vollmers, C., Zarrinpar, A., DiTacchio, L., Bushong, E.A., Gill, S., Leblanc, M., Chaix, A., Joens, M., Fitzpatrick, J.A., et al. (2012). Time-restricted feeding without reducing caloric intake prevents metabolic diseases in mice fed a high-fat diet. Cell Metab 15, 848-860.

Hattar, S., Lucas, R.J., Mrosovsky, N., Thompson, S., Douglas, R.H., Hankins, M.W., Lem, J., Biel, M., Hofmann, F., Foster, R.G., et al. (2003). Melanopsin and rod-cone photoreceptive systems account for all major accessory visual functions in mice. Nature $424,76-81$.

He, W., Barak, Y., Hevener, A., Olson, P., Liao, D., Le, J., Nelson, M., Ong, E., Olefsky, J.M., and Evans, R.M. (2003). Adipose-specific peroxisome proliferator-activated receptor gamma knockout causes insulin resistance in fat and liver but not in muscle. Proc Natl Acad Sci U S A 100, 1571215717.

Heckemeyer, C.M., Barker, J., Duckworth, W.C., and Solomon, S.S. (1983). Studies of the biological effect and degradation of glucagon in the rat perifused isolated adipose cell. Endocrinology $113,270-276$.

Hemmeryckx, B., Himmelreich, U., Hoylaerts, M.F., and Lijnen, H.R. (2011). Impact of clock gene Bmal1 deficiency on nutritionally induced obesity in mice. Obesity (Silver Spring) 19, 659-661.

Herichova, I., Zeman, M., Stebelova, K., and Ravingerova, T. (2005). Effect of streptozotocininduced diabetes on daily expression of per 2 and $\mathrm{dbp}$ in the heart and liver and melatonin rhythm in the pineal gland of Wistar rat. Mol Cell Biochem 270, 223-229.

Hermes, M.L., Coderre, E.M., Buijs, R.M., and Renaud, L.P. (1996). GABA and glutamate mediate rapid neurotransmission from suprachiasmatic nucleus to hypothalamic paraventricular nucleus in rat. J Physiol 496 ( Pt 3), 749-757.

Hirota, T., Lee, J.W., St John, P.C., Sawa, M., Iwaisako, K., Noguchi, T., Pongsawakul, P.Y., Sonntag, T., Welsh, D.K., Brenner, D.A., et al. (2012). Identification of small molecule activators of cryptochrome. Science 337, 1094-1097.

Hoang, N., Schleicher, E., Kacprzak, S., Bouly, J.P., Picot, M., Wu, W., Berndt, A., Wolf, E., Bittl, R., and Ahmad, M. (2008). Human and Drosophila cryptochromes are light activated by flavin photoreduction in living cells. PLoS Biol 6, e160.

Holm, C., Osterlund, T., Laurell, H., and Contreras, J.A. (2000). Molecular mechanisms regulating hormone-sensitive lipase and lipolysis. Annu Rev Nutr 20, 365-393.

Honma, K., von Goetz, C., and Aschoff, J. (1983). Effects of restricted daily feeding on freerunning circadian rhythms in rats. Physiol Behav 30, 905-913.

Honma, S., Ikeda, M., Abe, H., Tanahashi, Y., Namihira, M., Honma, K., and Nomura, M. (1998). Circadian oscillation of BMAL1, a partner of a mammalian clock gene Clock, in rat suprachiasmatic nucleus. Biochem Biophys Res Commun 250, 83-87. 
Honma, S., Kawamoto, T., Takagi, Y., Fujimoto, K., Sato, F., Noshiro, M., Kato, Y., and Honma, K. (2002). Dec1 and Dec2 are regulators of the mammalian molecular clock. Nature 419, 841-844.

Hu, E., Liang, P., and Spiegelman, B.M. (1996). AdipoQ is a novel adipose-specific gene dysregulated in obesity. J Biol Chem 271, 10697-10703.

Huijsman, E., van de Par, C., Economou, C., van der Poel, C., Lynch, G.S., Schoiswohl, G., Haemmerle, G., Zechner, R., and Watt, M.J. (2009). Adipose triacylglycerol lipase deletion alters whole body energy metabolism and impairs exercise performance in mice. Am J Physiol Endocrinol Metab 297, E505-513.

Hummel, K.P., Dickie, M.M., and Coleman, D.L. (1966). Diabetes, a new mutation in the mouse. Science 153, 1127-1128.

Hurd, M.W., and Ralph, M.R. (1998). The significance of circadian organization for longevity in the golden hamster. J Biol Rhythms 13, 430-436.

Husse, J., Zhou, X., Shostak, A., Oster, H., and Eichele, G. (2011). Synaptotagmin10-Cre, a driver to disrupt clock genes in the SCN. J Biol Rhythms 26, 379-389.

Ingalls, A.M., Dickie, M.M., and Snell, G.D. (1950). Obese, a new mutation in the house mouse. J Hered 41, 317-318.

Isojima, Y., Nakajima, M., Ukai, H., Fujishima, H., Yamada, R.G., Masumoto, K.H., Kiuchi, R., Ishida, M., Ukai-Tadenuma, M., Minami, Y., et al. (2009). CKlepsilon/delta-dependent phosphorylation is a temperature-insensitive, period-determining process in the mammalian circadian clock. Proc Natl Acad Sci U S A 106, 15744-15749.

Jeyaraj, D., Haldar, S.M., Wan, X., McCauley, M.D., Ripperger, J.A., Hu, K., Lu, Y., Eapen, B.L., Sharma, N., Ficker, E., et al. (2012a). Circadian rhythms govern cardiac repolarization and arrhythmogenesis. Nature 483, 96-99.

Jeyaraj, D., Scheer, F.A., Ripperger, J.A., Haldar, S.M., Lu, Y., Prosdocimo, D.A., Eapen, S.J., Eapen, B.L., Cui, Y., Mahabeleshwar, G.H., et al. (2012b). Klf15 orchestrates circadian nitrogen homeostasis. Cell Metab 15, 311-323.

Jud, C., Schmutz, I., Hampp, G., Oster, H., and Albrecht, U. (2005). A guideline for analyzing circadian wheel-running behavior in rodents under different lighting conditions. Biol Proced Online 7, 101-116.

Kalderon, B., Mayorek, N., Berry, E., Zevit, N., and Bar-Tana, J. (2000). Fatty acid cycling in the fasting rat. Am J Physiol Endocrinol Metab 279, E221-227.

Kalsbeek, A., Fliers, E., Romijn, J.A., La Fleur, S.E., Wortel, J., Bakker, O., Endert, E., and Buijs, R.M. (2001). The suprachiasmatic nucleus generates the diurnal changes in plasma leptin levels. Endocrinology 142, 2677-2685. 
Karatsoreos, I.N., Wang, A., Sasanian, J., and Silver, R. (2007). A role for androgens in regulating circadian behavior and the suprachiasmatic nucleus. Endocrinology 148, 5487-5495.

Karlsson, B., Knutsson, A., and Lindahl, B. (2001). Is there an association between shift work and having a metabolic syndrome? Results from a population based study of 27,485 people. Occup Environ Med 58, 747-752.

Katada, S., and Sassone-Corsi, P. (2010). The histone methyltransferase MLL1 permits the oscillation of circadian gene expression. Nat Struct Mol Biol 17, 1414-1421.

Kershaw, E.E., Schupp, M., Guan, H.P., Gardner, N.P., Lazar, M.A., and Flier, J.S. (2007). PPARgamma regulates adipose triglyceride lipase in adipocytes in vitro and in vivo. Am J Physiol Endocrinol Metab 293, E1736-1745.

Kim, J.Y., Tillison, K., Lee, J.H., Rearick, D.A., and Smas, C.M. (2006). The adipose tissue triglyceride lipase ATGL/PNPLA2 is downregulated by insulin and TNF-alpha in 3T3-L1 adipocytes and is a target for transactivation by PPARgamma. Am J Physiol Endocrinol Metab 291, E115-127.

Kleinridders, A., Schenten, D., Konner, A.C., Belgardt, B.F., Mauer, J., Okamura, T., Wunderlich, F.T., Medzhitov, R., and Bruning, J.C. (2009). MyD88 signaling in the CNS is required for development of fatty acid-induced leptin resistance and diet-induced obesity. Cell Metab 10, 249-259.

Kobayashi, K., Inoguchi, T., Maeda, Y., Nakashima, N., Kuwano, A., Eto, E., Ueno, N., Sasaki, S., Sawada, F., Fujii, M., et al. (2008). The lack of the C-terminal domain of adipose triglyceride lipase causes neutral lipid storage disease through impaired interactions with lipid droplets. J Clin Endocrinol Metab 93, 2877-2884.

Kohsaka, A., Laposky, A.D., Ramsey, K.M., Estrada, C., Joshu, C., Kobayashi, Y., Turek, F.W., and Bass, J. (2007). High-fat diet disrupts behavioral and molecular circadian rhythms in mice. Cell Metab $6,414-421$.

Kojetin, D., Wang, Y., Kamenecka, T.M., and Burris, T.P. (2011). Identification of SR8278, a synthetic antagonist of the nuclear heme receptor REV-ERB. ACS Chem Biol 6, 131-134.

Kondratov, R.V., Kondratova, A.A., Gorbacheva, V.Y., Vykhovanets, O.V., and Antoch, M.P. (2006). Early aging and age-related pathologies in mice deficient in BMAL1, the core componentof the circadian clock. Genes Dev 20, 1868-1873.

Konopka, R.J., and Benzer, S. (1971). Clock mutants of Drosophila melanogaster. Proc Natl Acad Sci U S A 68, 2112-2116.

Kornmann, B., Schaad, O., Bujard, H., Takahashi, J.S., and Schibler, U. (2007). System-driven and oscillator-dependent circadian transcription in mice with a conditionally active liver clock. PLoS Biol 5, e34. 
Kramer, A., Yang, F.C., Snodgrass, P., Li, X., Scammell, T.E., Davis, F.C., and Weitz, C.J. (2001). Regulation of daily locomotor activity and sleep by hypothalamic EGF receptor signaling. Science 294, 2511-2515.

Kraves, S., and Weitz, C.J. (2006). A role for cardiotrophin-like cytokine in the circadian control of mammalian locomotor activity. Nat Neurosci 9, 212-219.

Kumar, N., Solt, L.A., Wang, Y., Rogers, P.M., Bhattacharyya, G., Kamenecka, T.M., Stayrook, K.R., Crumbley, C., Floyd, Z.E., Gimble, J.M., et al. (2010). Regulation of adipogenesis by natural and synthetic REV-ERB ligands. Endocrinology 151, 3015-3025.

Kurosawa, G., and Iwasa, Y. (2005). Temperature compensation in circadian clock models. J Theor Biol 233, 453-468.

La Fleur, S.E., Kalsbeek, A., Wortel, J., and Buijs, R.M. (1999). A suprachiasmatic nucleus generated rhythm in basal glucose concentrations. J Neuroendocrinol 11, 643-652.

Lafontan, M., and Berlan, M. (1993). Fat cell adrenergic receptors and the control of white and brown fat cell function. J Lipid Res 34, 1057-1091.

Lamia, K.A., Papp, S.J., Yu, R.T., Barish, G.D., Uhlenhaut, N.H., Jonker, J.W., Downes, M., and Evans, R.M. (2011). Cryptochromes mediate rhythmic repression of the glucocorticoid receptor. Nature $480,552-556$.

Lamia, K.A., Sachdeva, U.M., DiTacchio, L., Williams, E.C., Alvarez, J.G., Egan, D.F., Vasquez, D.S., Juguilon, H., Panda, S., Shaw, R.J., et al. (2009). AMPK regulates the circadian clock by cryptochrome phosphorylation and degradation. Science $326,437-440$.

Lamia, K.A., Storch, K.F., and Weitz, C.J. (2008). Physiological significance of a peripheral tissue circadian clock. Proc Natl Acad Sci U S A 105, 15172-15177.

Langin, D. (2006). Control of fatty acid and glycerol release in adipose tissue lipolysis. C R Biol 329, 598-607; discussion 653-595.

Levine, J.D., Weiss, M.L., Rosenwasser, A.M., and Miselis, R.R. (1991). Retinohypothalamic tract in the female albino rat: a study using horseradish peroxidase conjugated to cholera toxin. J Comp Neurol 306, 344-360.

Lin, C., and Todo, T. (2005). The cryptochromes. Genome Biol 6, 220.

Liu, A.C., Welsh, D.K., Ko, C.H., Tran, H.G., Zhang, E.E., Priest, A.A., Buhr, E.D., Singer, O., Meeker, K., Verma, I.M., et al. (2007). Intercellular coupling confers robustness against mutations in the SCN circadian clock network. Cell 129, 605-616.

Londos, C., Cooper, D.M., Schlegel, W., and Rodbell, M. (1978). Adenosine analogs inhibit adipocyte adenylate cyclase by a GTP-dependent process: basis for actions of adenosine and methylxanthines on cyclic AMP production and lipolysis. Proc Natl Acad Sci U S A 75, 5362-5366. 
Lopez-Molina, L., Conquet, F., Dubois-Dauphin, M., and Schibler, U. (1997). The DBP gene is expressed according to a circadian rhythm in the suprachiasmatic nucleus and influences circadian behavior. EMBO J 16, 6762-6771.

Lu, W., Meng, Q.J., Tyler, N.J., Stokkan, K.A., and Loudon, A.S. (2010). A circadian clock is not required in an arctic mammal. Curr Biol 20, 533-537.

Maeda, K., Okubo, K., Shimomura, I., Funahashi, T., Matsuzawa, Y., and Matsubara, K. (1996). cDNA cloning and expression of a novel adipose specific collagen-like factor, apM1 (AdiPose Most abundant Gene transcript 1). Biochem Biophys Res Commun 221, 286-289.

Malek, Z.S., Sage, D., Pevet, P., and Raison, S. (2007). Daily rhythm of tryptophan hydroxylase-2 messenger ribonucleic acid within raphe neurons is induced by corticoid daily surge and modulated by enhanced locomotor activity. Endocrinology 148, 5165-5172.

Mamontova, A., Seguret-Mace, S., Esposito, B., Chaniale, C., Bouly, M., Delhaye-Bouchaud, N., Luc, G., Staels, B., Duverger, N., Mariani, J., et al. (1998). Severe atherosclerosis and hypoalphalipoproteinemia in the staggerer mouse, a mutant of the nuclear receptor RORalpha. Circulation 98, 2738-2743.

Marcheva, B., Ramsey, K.M., Buhr, E.D., Kobayashi, Y., Su, H., Ko, C.H., Ivanova, G., Omura, C., Mo, S., Vitaterna, M.H., et al. (2010). Disruption of the clock components CLOCK and BMAL1 leads to hypoinsulinaemia and diabetes. Nature 466, 627-631.

Meng, Q.J., Logunova, L., Maywood, E.S., Gallego, M., Lebiecki, J., Brown, T.M., Sladek, M., Semikhodskii, A.S., Glossop, N.R., Piggins, H.D., et al. (2008). Setting clock speed in mammals: the CK1 epsilon tau mutation in mice accelerates circadian pacemakers by selectively destabilizing PERIOD proteins. Neuron $58,78-88$.

Mieda, M., and Sakurai, T. (2011). Bmal1 in the nervous system is essential for normal adaptation of circadian locomotor activity and food intake to periodic feeding. J Neurosci 31, 1539115396.

Mitsui, S., Yamaguchi, S., Matsuo, T., Ishida, Y., and Okamura, H. (2001). Antagonistic role of E4BP4 and PAR proteins in the circadian oscillatory mechanism. Genes Dev 15, 995-1006.

Morin, L.P., Fitzgerald, K.M., and Zucker, I. (1977). Estradiol shortens the period of hamster circadian rhythms. Science 196, 305-307.

Nagoshi, E., Saini, C., Bauer, C., Laroche, T., Naef, F., and Schibler, U. (2004). Circadian gene expression in individual fibroblasts: cell-autonomous and self-sustained oscillators pass time to daughter cells. Cell 119, 693-705.

Nakahata, Y., Kaluzova, M., Grimaldi, B., Sahar, S., Hirayama, J., Chen, D., Guarente, L.P., and Sassone-Corsi, P. (2008). The NAD+-dependent deacetylase SIRT1 modulates CLOCK-mediated chromatin remodeling and circadian control. Cell 134, 329-340. 
Nakahata, Y., Sahar, S., Astarita, G., Kaluzova, M., and Sassone-Corsi, P. (2009). Circadian control of the NAD+ salvage pathway by CLOCK-SIRT1. Science 324, 654-657.

Nakahira, Y., Katayama, M., Miyashita, H., Kutsuna, S., Iwasaki, H., Oyama, T., and Kondo, T. (2004). Global gene repression by KaiC as a master process of prokaryotic circadian system. Proc Natl Acad Sci U S A 101, 881-885.

Nakajima, M., Imai, K., Ito, H., Nishiwaki, T., Murayama, Y., Iwasaki, H., Oyama, T., and Kondo, T. (2005). Reconstitution of circadian oscillation of cyanobacterial KaiC phosphorylation in vitro. Science $308,414-415$.

Nakamura, T.J., Moriya, T., Inoue, S., Shimazoe, T., Watanabe, S., Ebihara, S., and Shinohara, K. (2005). Estrogen differentially regulates expression of Per1 and Per2 genes between central and peripheral clocks and between reproductive and nonreproductive tissues in female rats. J Neurosci Res 82, 622-630.

Nakano, Y., Tobe, T., Choi-Miura, N.H., Mazda, T., and Tomita, M. (1996). Isolation and characterization of GBP28, a novel gelatin-binding protein purified from human plasma. J Biochem $120,803-812$.

O'Neill, J.S., and Reddy, A.B. (2011). Circadian clocks in human red blood cells. Nature 469, 498-503.

O'Neill, J.S., van Ooijen, G., Dixon, L.E., Troein, C., Corellou, F., Bouget, F.Y., Reddy, A.B., and Millar, A.J. (2011). Circadian rhythms persist without transcription in a eukaryote. Nature 469, 554558.

Oishi, K., Kasamatsu, M., and Ishida, N. (2004). Gene- and tissue-specific alterations of circadian clock gene expression in streptozotocin-induced diabetic mice under restricted feeding. Biochem Biophys Res Commun 317, 330-334.

Oster, H., Baeriswyl, S., Van Der Horst, G.T., and Albrecht, U. (2003). Loss of circadian rhythmicity in aging mPer1-/-mCry2-/- mutant mice. Genes Dev 17, 1366-1379.

Oster, H., Damerow, S., Kiessling, S., Jakubcakova, V., Abraham, D., Tian, J., Hoffmann, M.W., and Eichele, G. (2006). The circadian rhythm of glucocorticoids is regulated by a gating mechanism residing in the adrenal cortical clock. Cell Metab 4, 163-173.

Osuga, J., Ishibashi, S., Oka, T., Yagyu, H., Tozawa, R., Fujimoto, A., Shionoiri, F., Yahagi, N., Kraemer, F.B., Tsutsumi, O., et al. (2000). Targeted disruption of hormone-sensitive lipase results in male sterility and adipocyte hypertrophy, but not in obesity. Proc Natl Acad Sci U S A 97, 787-792.

Otway, D.T., Mantele, S., Bretschneider, S., Wright, J., Trayhurn, P., Skene, D.J., Robertson, M.D., and Johnston, J.D. (2011). Rhythmic diurnal gene expression in human adipose tissue from individuals who are lean, overweight, and type 2 diabetic. Diabetes 60, 1577-1581. 
Ouyang, Y., Andersson, C.R., Kondo, T., Golden, S.S., and Johnson, C.H. (1998). Resonating circadian clocks enhance fitness in cyanobacteria. Proc Natl Acad Sci U S A 95, 8660-8664.

Pagnon, J., Matzaris, M., Stark, R., Meex, R.C., Macaulay, S.L., Brown, W., O'Brien, P.E., Tiganis, T., and Watt, M.J. (2012). Identification and functional characterization of protein kinase a phosphorylation sites in the major lipolytic protein, adipose triglyceride lipase. Endocrinology 153, 4278-4289.

Pan, A., Schernhammer, E.S., Sun, Q., and Hu, F.B. (2011). Rotating night shift work and risk of type 2 diabetes: two prospective cohort studies in women. PLoS Med 8, e1001141.

Panda, S., Antoch, M.P., Miller, B.H., Su, A.I., Schook, A.B., Straume, M., Schultz, P.G., Kay, S.A., Takahashi, J.S., and Hogenesch, J.B. (2002). Coordinated transcription of key pathways in the mouse by the circadian clock. Cell 109, 307-320.

Peers, D.G., and Davies, J.I. (1971). Significance of the caffeine-like effect of various purines, pyrimidines and derivatives on adipose-tissue phosphodiesterase. Biochem J 124, 8P-9P.

Perea, A., Clemente, F., Martinell, J., Villanueva-Penacarrillo, M.L., and Valverde, I. (1995). Physiological effect of glucagon in human isolated adipocytes. Horm Metab Res 27, 372-375.

Peterfy, M., Phan, J., Xu, P., and Reue, K. (2001). Lipodystrophy in the fld mouse results from mutation of a new gene encoding a nuclear protein, lipin. Nat Genet 27, 121-124.

Pittendrigh, C.S. (1954). On Temperature Independence in the Clock System Controlling Emergence Time in Drosophila. Proc Natl Acad Sci U S A 40, 1018-1029.

Pittendrigh, C.S. (1993). Temporal organization: reflections of a Darwinian clock-watcher. Annu Rev Physiol 55, 16-54.

Preitner, N., Damiola, F., Lopez-Molina, L., Zakany, J., Duboule, D., Albrecht, U., and Schibler, U. (2002). The orphan nuclear receptor REV-ERBalpha controls circadian transcription within the positive limb of the mammalian circadian oscillator. Cell 110, 251-260.

Ptitsyn, A.A., Zvonic, S., Conrad, S.A., Scott, L.K., Mynatt, R.L., and Gimble, J.M. (2006). Circadian clocks are resounding in peripheral tissues. PLoS Comput Biol 2, e16.

Ralph, M.R., Foster, R.G., Davis, F.C., and Menaker, M. (1990). Transplanted suprachiasmatic nucleus determines circadian period. Science 247, 975-978.

Ralph, M.R., and Menaker, M. (1988). A mutation of the circadian system in golden hamsters. Science $241,1225-1227$.

Ramsey, K.M., Yoshino, J., Brace, C.S., Abrassart, D., Kobayashi, Y., Marcheva, B., Hong, H.K., Chong, J.L., Buhr, E.D., Lee, C., et al. (2009). Circadian clock feedback cycle through NAMPT-mediated NAD+ biosynthesis. Science 324, 651-654. 
Raspe, E., Duez, H., Mansen, A., Fontaine, C., Fievet, C., Fruchart, J.C., Vennstrom, B., and Staels, B. (2002). Identification of Rev-erbalpha as a physiological repressor of apoC-III gene transcription. J Lipid Res 43, 2172-2179.

Ravussin, E., and Galgani, J.E. (2011). The implication of brown adipose tissue for humans. Annu Rev Nutr 31, 33-47.

Razani, B., Wang, X.B., Engelman, J.A., Battista, M., Lagaud, G., Zhang, X.L., Kneitz, B., Hou, H., Jr., Christ, G.J., Edelmann, W., et al. (2002). Caveolin-2-deficient mice show evidence of severe pulmonary dysfunction without disruption of caveolae. Mol Cell Biol 22, 2329-2344.

Redlin, U., Nuesslein, B., and Schmidt, I. (1992). Circadian changes of brown adipose tissue thermogenesis in juvenile rats. Am J Physiol 262, R504-508.

Rongvaux, A., Shea, R.J., Mulks, M.H., Gigot, D., Urbain, J., Leo, O., and Andris, F. (2002). Pre-Bcell colony-enhancing factor, whose expression is up-regulated in activated lymphocytes, is a nicotinamide phosphoribosyltransferase, a cytosolic enzyme involved in NAD biosynthesis. Eur J Immunol 32, 3225-3234.

Rossner, M.J., Oster, H., Wichert, S.P., Reinecke, L., Wehr, M.C., Reinecke, J., Eichele, G., Taneja, R., and Nave, K.A. (2008). Disturbed clockwork resetting in Sharp-1 and Sharp-2 single and double mutant mice. PLoS One 3, e2762.

Rudic, R.D., McNamara, P., Curtis, A.M., Boston, R.C., Panda, S., Hogenesch, J.B., and Fitzgerald, G.A. (2004). BMAL1 and CLOCK, two essential components of the circadian clock, are involved in glucose homeostasis. PLoS Biol 2, e377.

Ruoff, P., Rensing, L., Kommedal, R., and Mohsenzadeh, S. (1997). Modeling temperature compensation in chemical and biological oscillators. Chronobiol Int 14, 499-510.

Ryden, M., Arvidsson, E., Blomqvist, L., Perbeck, L., Dicker, A., and Arner, P. (2004). Targets for TNF-alpha-induced lipolysis in human adipocytes. Biochem Biophys Res Commun 318, 168-175.

Ryden, M., Dicker, A., van Harmelen, V., Hauner, H., Brunnberg, M., Perbeck, L., Lonnqvist, F., and Arner, P. (2002). Mapping of early signaling events in tumor necrosis factor-alpha -mediated lipolysis in human fat cells. J Biol Chem 277, 1085-1091.

Ryden, M., Jocken, J., van Harmelen, V., Dicker, A., Hoffstedt, J., Wiren, M., Blomqvist, L., Mairal, A., Langin, D., Blaak, E., et al. (2007). Comparative studies of the role of hormone-sensitive lipase and adipose triglyceride lipase in human fat cell lipolysis. Am J Physiol Endocrinol Metab 292, E1847-1855.

Sadacca, L.A., Lamia, K.A., deLemos, A.S., Blum, B., and Weitz, C.J. (2011). An intrinsic circadian clock of the pancreas is required for normal insulin release and glucose homeostasis in mice.

Diabetologia 54, 120-124. 
Sahar, S., and Sassone-Corsi, P. (2012). Regulation of metabolism: the circadian clock dictates the time. Trends Endocrinol Metab 23, 1-8.

Samal, B., Sun, Y., Stearns, G., Xie, C., Suggs, S., and McNiece, I. (1994). Cloning and characterization of the cDNA encoding a novel human pre-B-cell colony-enhancing factor. Mol Cell Biol 14, 1431-1437.

Sato, T.K., Panda, S., Miraglia, L.J., Reyes, T.M., Rudic, R.D., McNamara, P., Naik, K.A., FitzGerald, G.A., Kay, S.A., and Hogenesch, J.B. (2004). A functional genomics strategy reveals Rora as a component of the mammalian circadian clock. Neuron 43, 527-537.

Satoh, Y., Kawai, H., Kudo, N., Kawashima, Y., and Mitsumoto, A. (2006). Time-restricted feeding entrains daily rhythms of energy metabolism in mice. Am J Physiol Regul Integr Comp Physiol 290, R1276-1283.

Scheer, F.A., Hilton, M.F., Mantzoros, C.S., and Shea, S.A. (2009). Adverse metabolic and cardiovascular consequences of circadian misalignment. Proc Natl Acad Sci U S A 106, 4453-4458.

Scherer, P.E., Williams, S., Fogliano, M., Baldini, G., and Lodish, H.F. (1995). A novel serum protein similar to C1q, produced exclusively in adipocytes. J Biol Chem 270, 26746-26749.

Schweiger, M., Schreiber, R., Haemmerle, G., Lass, A., Fledelius, C., Jacobsen, P., Tornqvist, H., Zechner, R., and Zimmermann, R. (2006). Adipose triglyceride lipase and hormone-sensitive lipase are the major enzymes in adipose tissue triacylglycerol catabolism. J Biol Chem 281, 40236-40241.

Scott, E.M., Carter, A.M., and Grant, P.J. (2008). Association between polymorphisms in the Clock gene, obesity and the metabolic syndrome in man. Int J Obes (Lond) 32, 658-662.

Shimba, S., Ishii, N., Ohta, Y., Ohno, T., Watabe, Y., Hayashi, M., Wada, T., Aoyagi, T., and Tezuka, M. (2005). Brain and muscle Arnt-like protein-1 (BMAL1), a component of the molecular clock, regulates adipogenesis. Proc Natl Acad Sci U S A 102, 12071-12076.

Shimba, S., Ogawa, T., Hitosugi, S., Ichihashi, Y., Nakadaira, Y., Kobayashi, M., Tezuka, M., Kosuge, Y., Ishige, K., Ito, Y., et al. (2011). Deficient of a clock gene, brain and muscle Arnt-like protein-1 (BMAL1), induces dyslipidemia and ectopic fat formation. PLoS One 6, e25231.

Sinha, M.K., Ohannesian, J.P., Heiman, M.L., Kriauciunas, A., Stephens, T.W., Magosin, S., Marco, C., and Caro, J.F. (1996). Nocturnal rise of leptin in lean, obese, and non-insulin-dependent diabetes mellitus subjects. J Clin Invest 97, 1344-1347.

Smih, F., Rouet, P., Lucas, S., Mairal, A., Sengenes, C., Lafontan, M., Vaulont, S., Casado, M., and Langin, D. (2002). Transcriptional regulation of adipocyte hormone-sensitive lipase by glucose. Diabetes 51, 293-300.

Smith, S.J., Cases, S., Jensen, D.R., Chen, H.C., Sande, E., Tow, B., Sanan, D.A., Raber, J., Eckel, R.H., and Farese, R.V., Jr. (2000). Obesity resistance and multiple mechanisms of triglyceride synthesis in mice lacking Dgat. Nat Genet 25, 87-90. 
Solt, L.A., Wang, Y., Banerjee, S., Hughes, T., Kojetin, D.J., Lundasen, T., Shin, Y., Liu, J., Cameron, M.D., Noel, R., et al. (2012). Regulation of circadian behaviour and metabolism by synthetic REV-ERB agonists. Nature 485, 62-68.

Sookoian, S., Gemma, C., Gianotti, T.F., Burgueno, A., Castano, G., and Pirola, C.J. (2008). Genetic variants of Clock transcription factor are associated with individual susceptibility to obesity. Am J Clin Nutr 87, 1606-1615.

Spiegel, K., Tasali, E., Penev, P., and Van Cauter, E. (2004). Brief communication: Sleep curtailment in healthy young men is associated with decreased leptin levels, elevated ghrelin levels, and increased hunger and appetite. Ann Intern Med 141, 846-850.

Stephan, F.K., and Zucker, I. (1972). Circadian rhythms in drinking behavior and locomotor activity of rats are eliminated by hypothalamic lesions. Proc Natl Acad Sci U S A 69, 1583-1586.

Stokkan, K.A., Yamazaki, S., Tei, H., Sakaki, Y., and Menaker, M. (2001). Entrainment of the circadian clock in the liver by feeding. Science $291,490-493$.

Stone, S.J., Myers, H.M., Watkins, S.M., Brown, B.E., Feingold, K.R., Elias, P.M., and Farese, R.V., Jr. (2004). Lipopenia and skin barrier abnormalities in DGAT2-deficient mice. J Biol Chem 279, 11767-11776.

Storch, K.F., Lipan, O., Leykin, I., Viswanathan, N., Davis, F.C., Wong, W.H., and Weitz, C.J. (2002). Extensive and divergent circadian gene expression in liver and heart. Nature 417, 78-83.

Storch, K.F., Paz, C., Signorovitch, J., Raviola, E., Pawlyk, B., Li, T., and Weitz, C.J. (2007). Intrinsic circadian clock of the mammalian retina: importance for retinal processing of visual information. Cell 130, 730-741.

Stow, L.R., Richards, J., Cheng, K.Y., Lynch, I.J., Jeffers, L.A., Greenlee, M.M., Cain, B.D., Wingo, C.S., and Gumz, M.L. (2012). The circadian protein period 1 contributes to blood pressure control and coordinately regulates renal sodium transport genes. Hypertension 59, 1151-1156.

Sun, Z.S., Albrecht, U., Zhuchenko, O., Bailey, J., Eichele, G., and Lee, C.C. (1997). RIGUI, a putative mammalian ortholog of the Drosophila period gene. Cell 90, 1003-1011.

Taheri, S., Lin, L., Austin, D., Young, T., and Mignot, E. (2004). Short sleep duration is associated with reduced leptin, elevated ghrelin, and increased body mass index. PLoS Med 1, e62.

Tei, H., Okamura, H., Shigeyoshi, Y., Fukuhara, C., Ozawa, R., Hirose, M., and Sakaki, Y. (1997). Circadian oscillation of a mammalian homologue of the Drosophila period gene. Nature $389,512-$ 516.

Thorens, B., Flier, J.S., Lodish, H.F., and Kahn, B.B. (1990). Differential regulation of two glucose transporters in rat liver by fasting and refeeding and by diabetes and insulin treatment. Diabetes 39 , 712-719. 
Tomita, J., Nakajima, M., Kondo, T., and Iwasaki, H. (2005). No transcription-translation feedback in circadian rhythm of KaiC phosphorylation. Science 307, 251-254.

Trayhurn, P. (2007). Adipocyte biology. Obes Rev 8 Suppl 1, 41-44.

Trujillo, M.E., and Scherer, P.E. (2005). Adiponectin--journey from an adipocyte secretory protein to biomarker of the metabolic syndrome. J Intern Med 257, 167-175.

Turek, F.W., Joshu, C., Kohsaka, A., Lin, E., Ivanova, G., McDearmon, E., Laposky, A., LoseeOlson, S., Easton, A., Jensen, D.R., et al. (2005). Obesity and metabolic syndrome in circadian Clock mutant mice. Science 308, 1043-1045.

Unger, R.H., Clark, G.O., Scherer, P.E., and Orci, L. (2010). Lipid homeostasis, lipotoxicity and the metabolic syndrome. Biochim Biophys Acta 1801, 209-214.

van der Horst, G.T., Muijtjens, M., Kobayashi, K., Takano, R., Kanno, S., Takao, M., de Wit, J., Verkerk, A., Eker, A.P., van Leenen, D., et al. (1999). Mammalian Cry1 and Cry2 are essential for maintenance of circadian rhythms. Nature 398, 627-630.

van Oort, B.E., Tyler, N.J., Gerkema, M.P., Folkow, L., Blix, A.S., and Stokkan, K.A. (2005). Circadian organization in reindeer. Nature 438, 1095-1096.

Veerman, D.P., Imholz, B.P., Wieling, W., Wesseling, K.H., and van Montfrans, G.A. (1995). Circadian profile of systemic hemodynamics. Hypertension 26, 55-59.

Vitaterna, M.H., King, D.P., Chang, A.M., Kornhauser, J.M., Lowrey, P.L., McDonald, J.D., Dove, W.F., Pinto, L.H., Turek, F.W., and Takahashi, J.S. (1994). Mutagenesis and mapping of a mouse gene, Clock, essential for circadian behavior. Science 264, 719-725.

Vogel, P., Read, R., Hansen, G., Wingert, J., Dacosta, C.M., Buhring, L.M., and Shadoan, M. (2011). Pathology of congenital generalized lipodystrophy in Agpat2-/- mice. Vet Pathol 48, 642-654.

Vollmers, C., Gill, S., DiTacchio, L., Pulivarthy, S.R., Le, H.D., and Panda, S. (2009). Time of feeding and the intrinsic circadian clock drive rhythms in hepatic gene expression. Proc Natl Acad Sci U S A 106, 21453-21458.

Vorona, R.D., Winn, M.P., Babineau, T.W., Eng, B.P., Feldman, H.R., and Ware, J.C. (2005). Overweight and obese patients in a primary care population report less sleep than patients with a normal body mass index. Arch Intern Med 165, 25-30.

Vujovic, N., Davidson, A.J., and Menaker, M. (2008). Sympathetic input modulates, but does not determine, phase of peripheral circadian oscillators. Am J Physiol Regul Integr Comp Physiol 295, R355-360.

Wang, J., and Lazar, M.A. (2008). Bifunctional role of Rev-erbalpha in adipocyte differentiation. Mol Cell Biol 28, 2213-2220. 
Wang, S.P., Laurin, N., Himms-Hagen, J., Rudnicki, M.A., Levy, E., Robert, M.F., Pan, L., Oligny, L., and Mitchell, G.A. (2001). The adipose tissue phenotype of hormone-sensitive lipase deficiency in mice. Obes Res 9, 119-128.

Welsh, D.K., Takahashi, J.S., and Kay, S.A. (2010). Suprachiasmatic nucleus: cell autonomy and network properties. Annu Rev Physiol 72, 551-577.

Welsh, D.K., Yoo, S.H., Liu, A.C., Takahashi, J.S., and Kay, S.A. (2004). Bioluminescence imaging of individual fibroblasts reveals persistent, independently phased circadian rhythms of clock gene expression. Curr Biol 14, 2289-2295.

Woelfle, M.A., Ouyang, Y., Phanvijhitsiri, K., and Johnson, C.H. (2004). The adaptive value of circadian clocks: an experimental assessment in cyanobacteria. Curr Biol 14, 1481-1486.

Woon, P.Y., Kaisaki, P.J., Braganca, J., Bihoreau, M.T., Levy, J.C., Farrall, M., and Gauguier, D. (2007). Aryl hydrocarbon receptor nuclear translocator-like (BMAL1) is associated with susceptibility to hypertension and type 2 diabetes. Proc Natl Acad Sci U S A 104, 14412-14417.

Wozniak, S.E., Gee, L.L., Wachtel, M.S., and Frezza, E.E. (2009). Adipose tissue: the new endocrine organ? A review article. Dig Dis Sci 54, 1847-1856.

Wu, J.W., Wang, S.P., Casavant, S., Moreau, A., Yang, G.S., and Mitchell, G.A. (2012). Fasting energy homeostasis in mice with adipose deficiency of desnutrin/adipose triglyceride lipase. Endocrinology 153, 2198-2207.

Wu, Q., Ortegon, A.M., Tsang, B., Doege, H., Feingold, K.R., and Stahl, A. (2006). FATP1 is an insulin-sensitive fatty acid transporter involved in diet-induced obesity. Mol Cell Biol 26, 3455-3467.

Yagita, K., Tamanini, F., van Der Horst, G.T., and Okamura, H. (2001). Molecular mechanisms of the biological clock in cultured fibroblasts. Science 292, 278-281.

Yamamoto, T., Nakahata, Y., Soma, H., Akashi, M., Mamine, T., and Takumi, T. (2004). Transcriptional oscillation of canonical clock genes in mouse peripheral tissues. BMC Mol Biol 5, 18.

Yamazaki, S., Numano, R., Abe, M., Hida, A., Takahashi, R., Ueda, M., Block, G.D., Sakaki, Y., Menaker, M., and Tei, H. (2000). Resetting central and peripheral circadian oscillators in transgenic rats. Science $288,682-685$.

Yamazaki, S., and Takahashi, J.S. (2005). Real-time luminescence reporting of circadian gene expression in mammals. Methods Enzymol 393, 288-301.

Yang, S., Liu, A., Weidenhammer, A., Cooksey, R.C., McClain, D., Kim, M.K., Aguilera, G., Abel, E.D., and Chung, J.H. (2009). The role of mPer2 clock gene in glucocorticoid and feeding rhythms. Endocrinology 150, 2153-2160.

Yang, X., Downes, M., Yu, R.T., Bookout, A.L., He, W., Straume, M., Mangelsdorf, D.J., and Evans, R.M. (2006). Nuclear receptor expression links the circadian clock to metabolism. Cell 126, 801-810. 
Yi, C.X., van der Vliet, J., Dai, J., Yin, G., Ru, L., and Buijs, R.M. (2006). Ventromedial arcuate nucleus communicates peripheral metabolic information to the suprachiasmatic nucleus. Endocrinology 147, 283-294.

Yoo, S.H., Yamazaki, S., Lowrey, P.L., Shimomura, K., Ko, C.H., Buhr, E.D., Siepka, S.M., Hong, H.K., Oh, W.J., Yoo, O.J., et al. (2004). PERIOD2::LUCIFERASE real-time reporting of circadian dynamics reveals persistent circadian oscillations in mouse peripheral tissues. Proc Natl Acad Sci U S A $101,5339-5346$.

Zechner, R., Zimmermann, R., Eichmann, T.O., Kohlwein, S.D., Haemmerle, G., Lass, A., and Madeo, F. (2012). FAT SIGNALS--lipases and lipolysis in lipid metabolism and signaling. Cell Metab 15, 279-291.

Zhang, E.E., Liu, Y., Dentin, R., Pongsawakul, P.Y., Liu, A.C., Hirota, T., Nusinow, D.A., Sun, X., Landais, S., Kodama, Y., et al. (2010). Cryptochrome mediates circadian regulation of cAMP signaling and hepatic gluconeogenesis. Nat Med 16, 1152-1156.

Zhang, Y., Proenca, R., Maffei, M., Barone, M., Leopold, L., and Friedman, J.M. (1994). Positional cloning of the mouse obese gene and its human homologue. Nature 372, 425-432.

Zheng, B., Larkin, D.W., Albrecht, U., Sun, Z.S., Sage, M., Eichele, G., Lee, C.C., and Bradley, A. (1999). The mPer2 gene encodes a functional component of the mammalian circadian clock. Nature $400,169-173$.

Zimmermann, R., Haemmerle, G., Wagner, E.M., Strauss, J.G., Kratky, D., and Zechner, R. (2003). Decreased fatty acid esterification compensates for the reduced lipolytic activity in hormonesensitive lipase-deficient white adipose tissue. J Lipid Res 44, 2089-2099.

Zuber, A.M., Centeno, G., Pradervand, S., Nikolaeva, S., Maquelin, L., Cardinaux, L., Bonny, O., and Firsov, D. (2009). Molecular clock is involved in predictive circadian adjustment of renal function. Proc Natl Acad Sci U S A 106, 16523-16528.

Zvonic, S., Ptitsyn, A.A., Conrad, S.A., Scott, L.K., Floyd, Z.E., Kilroy, G., Wu, X., Goh, B.C., Mynatt, R.L., and Gimble, J.M. (2006). Characterization of peripheral circadian clocks in adipose tissues. Diabetes 55, 962-970. 


\section{Additional publications}

Publication: Diurnal rhythm of circulating Nicotinamide

Phosphoribosyltransferase (Nampt/Visfatin/PBEF): impact of sleep loss and relation to glucose metabolism

Christian Benedict, Anton Shostak, Tanja Lange, Samantha J. Brooks, Helgi B. Schiöth, Bernd Schultes, Jan Born, Henrik Oster, and Manfred Hallschmid

The Journal of Clinical Endocrinology \& Metabolism, Volume 97, pages E218-E222

Anton Shostak performed visfatin ELISA for the Figure 1 and revised the manuscript. 


\title{
Diurnal Rhythm of Circulating Nicotinamide Phosphoribosyltransferase (Nampt/Visfatin/PBEF): Impact of Sleep Loss and Relation to Glucose Metabolism
}

\author{
Christian Benedict, Anton Shostak, Tanja Lange, Samantha J. Brooks, \\ Helgi B. Schiöth, Bernd Schultes, Jan Born, Henrik Oster, and \\ Manfred Hallschmid \\ Department of Neuroscience (C.B., S.J.B., H.B.S.), Uppsala University, SE-751 24 Uppsala, Sweden; Max \\ Planck Institute for Biophysical Chemistry (A.S., H.O.), D-37077 Göttingen, Germany; Department of \\ Neuroendocrinology (T.L., M.H.), University of Lübeck, E-23562 Lübeck, Germany; Interdisciplinary Obesity \\ Center (B.S.), Cantonal Hospital St. Gallen, CH-9007 St. Gallen, Switzerland; and Institute of Medical \\ Psychology and Behavioral Neurobiology (J.B.), University of Tübingen, D-72074 Tübingen, Germany
}

\begin{abstract}
Context: Animal studies indicate that nicotinamide phosphoribosyltransferase [Nampt/visfatin/ pre-B-cell colony-enhancing factor (PBEF)] contributes to the circadian fine-tuning of metabolic turnover. However, it is unknown whether circulating Nampt concentrations, which are elevated in type 2 diabetes and obesity, display a diurnal rhythm in humans.
\end{abstract}

Objective: Our objective was to examine the 24-h profile of serum Nampt in humans under conditions of sleep and sleep deprivation and relate the Nampt pattern to morning postprandial glucose metabolism.

Intervention: Fourteen healthy men participated in two 24 -h sessions starting at $1800 \mathrm{~h}$, including either regular 8-h-night sleep or continuous wakefulness. Serum Nampt and leptin were measured in 1.5- to 3-h intervals. In the morning, plasma glucose and serum insulin responses to standardized breakfast intake were determined.

Main Outcome Measures: Under regular sleep-wake conditions, Nampt levels displayed a pronounced diurnal rhythm, peaking during early afternoon $(P<0.001)$ that was inverse to leptin profiles peaking in the early night. When subjects stayed awake, the Nampt rhythm was preserved but phase advanced by about $2 \mathrm{~h}(P<0.05)$. Two-hour postprandial plasma glucose concentrations were elevated after sleep loss $(P<0.05)$, whereas serum insulin was not affected. The relative glucose increase due to sleep loss displayed a positive association with the magnitude of the Nampt phase shift $(r=0.54 ; P<0.05)$.

Conclusions: Serum Nampt concentrations follow a diurnal rhythm, peaking in the afternoon. Sleep loss induces a Nampt rhythm phase shift that is positively related to the impairment of postprandial glucose metabolism due to sleep deprivation, suggesting a regulatory impact of Nampt rhythmicity on glucose homeostasis. (J Clin Endocrinol Metab 97: E0000-E0000, 2012)

N cotinamide phosphoribosyltransferase (Nampt) is a multifunctional protein also known as visfatin and pre-B-cell colony-enhancing factor (PBEF) that is produced by adipose tissue as well as skeletal muscle, liver, and immune cells (1). Circulating Nampt concentrations have been reported to be elevated in type 2 diabetes $(2,3)$ and obesity $(4,5)$, supporting the recent assumption that the protein may be a marker of low-grade inflammation 
associated with metabolic dysfunction (6). In mice, the protein has been shown to act as a rate-limiting enzyme in the regulation of nicotinamide adenine dinucleotide, an essential coenzyme catalyzing ATP synthesis (7). Because the expression of Nampt in rodent adipocytes and hepatocytes shows a rhythmic 24-h pattern, the protein has been proposed to mediate a circadian feedback loop finetuning the integration of energy storage with the rest-activity cycle (7). Recent cross-sectional data from a human cohort indicate an inverse relationship between sleep duration and serum Nampt levels assessed in the morning (8), raising the question of whether circulating Nampt concentrations display a diurnal rhythm in humans and, if so, to which extent this rhythm is dependent on the sleep/ wake cycle. Furthermore, in light of the association between Nampt concentrations and metabolic dysfunctions (2-5), it might be hypothesized that sleep loss-induced alterations in Nampt signaling are related to the detrimental effects that compromised sleep exerts on glucose tolerance $(9,10)$. Against this background, we investigated the diurnal (i.e. 24-h) profile of serum Nampt in healthy humans under regular and sleep-deprivation conditions and assessed whether sleep deprivation-induced changes in serum Nampt concentrations are linked to impairments in morning glucose metabolism arising from sleep loss. We also compared Nampt patterns to those of leptin, an adipocytokine with a circadian secretion rhythm previously found to be sensitive to sleep loss (11).

\section{Subjects and Methods}

\section{Participants}

Fourteen healthy nonsmoking male subjects (mean \pm SEM, age $=22.6 \pm 0.8 \mathrm{yr}$; body mass index $=23.9 \pm 0.5 \mathrm{~kg} / \mathrm{m}^{2}$ ) participated in the experiments. All subjects had a regular selfreported sleep-wake rhythm during the $6 \mathrm{wk}$ before the experiments and were not on medication. Acute illness was excluded by a physical examination and routine laboratory testing. In the week before each experiment, subjects were instructed to go to bed between 2300 and $2330 \mathrm{~h}$ and to get up by $0700 \mathrm{~h}$ on the next morning and not to take any naps during the day. Sleep disturbances were excluded by monitoring sleep patterns in a separate adaptation night that also served to habituate subjects to the experimental setting. All subjects gave written informed consent to the study that conformed to the Declaration of Helsinki and was approved by the local ethics committee.

\section{Experimental protocol}

According to a randomized, balanced crossover design, each subject participated in two 24-h conditions [sleep and total sleep deprivation (TSD)] separated by $4 \mathrm{wk}$. Body weight did not differ between the Sleep and TSD conditions $(82.7 \pm 2.2 v s .83 .0 \pm 2.3$ $\mathrm{kg} ; P>0.1)$. Sessions started with a baseline period at $1800 \mathrm{~h}$ followed by the nocturnal intervention period $(2300-0700 \mathrm{~h})$ in which subjects slept or stayed awake and a postintervention pe- riod (0700-1800 h). During each experimental session, subjects ate standard meals at $1930 \mathrm{~h}(\sim 1700 \mathrm{~kJ}), 0830 \mathrm{~h}(\sim 3800 \mathrm{~kJ})$, and $1330 \mathrm{~h}(\sim 4500 \mathrm{~kJ})$. The liquid test breakfast at $0830 \mathrm{~h}$ contained $112.8 \mathrm{~g}$ carbohydrate (Fresubin energy drink; Fresenius Kabi, Bad Homburg, Germany). To minimize the biasing influence of spontaneous physical activity on measurements of energy expenditure conducted by indirect calorimetry (ventilated-hood system) at $0745 \mathrm{~h}$ and on an hourly basis between 0900 and $1300 \mathrm{~h}$ (see Ref. 12 for details and results), participants rested in bed in a supine position until $1300 \mathrm{~h}$ on the second day after which they changed to a sitting position until the end of the experiment. In the sleep condition, lights were turned off at $2300 \mathrm{~h}$, and subjects were awakened between 0630 and $0700 \mathrm{~h}$ when entering light sleep (i.e. sleep stage 1 or 2 ). Sleep recordings were performed according to standard criteria (13). In the TSD condition, lights were on ( $\sim 300$ lux $)$ and subjects were kept awake and continuously monitored by the experimenters. They were allowed to spend the night with a selection of nonarousing movies, games, and books. Drinking water was provided ad libitum, but food intake was not allowed.

\section{Assessments}

For the assessment of serum concentrations of Nampt, leptin, and insulin and of plasma glucose, blood was sampled in 1.5 - to 3-h intervals across the 24-h period. During sleep, blood was drawn via an iv forearm catheter connected to a long thin tube that enabled blood collection from an adjacent room without disturbing the subject's sleep. Blood samples were immediately centrifuged and frozen at $-80 \mathrm{C}$ until analysis. Nampt was analyzed using a commercially available enzyme immunoassay (Human Visfatin ELISA Kit; Enzo Life Sciences, Lörrach, Germany; intr-assay coefficient of variation $<10 \%$ ). Routine assays were used for the determination of serum leptin (Linco Research, St. Charles, MO), serum insulin (Siemens, Los Angeles, CA), and fluoride plasma glucose (Abbott, Abbott Park, IL). Analyses of insulin and glucose covered the breakfast period $(0730$ and $1100 \mathrm{~h}$ ) to reflect postprandial glucose metabolism in the morning (for respective 24-h profiles, see Ref. 12).

\section{Statistical analysis}

Data are presented as means \pm SEM. CircWave was used for rhythmicity analyses (14) (see Table 1 for details). Differences in pre- and postprandial glucose and insulin concentrations were specified by Student's $t$ tests. Spearman's rank correlations were calculated to detect associations between TSD-induced changes in Nampt rhythmicity and postprandial glucose concentrations. A $P$ value $<0.05$ was considered significant.

\section{Results}

\section{The 24-h profiles of serum Nampt and leptin}

In the sleep condition, when subjects adhered to a regular sleep-wake cycle, serum Nampt concentrations followed a significant diurnal rhythm peaking in the afternoon $(P<0.001$; Fig. $1 \mathrm{~A}$ and Table 1$)$. When subjects stayed awake throughout the night in the TSD condition, this rhythm was preserved $(P<0.05)$ but phase advanced by about $2 \mathrm{~h}$ in comparison with the sleep condition and 
TABLE 1. Curve-fitting statistics

\begin{tabular}{|c|c|c|c|c|c|c|c|c|c|}
\hline Hormone & Condition & $\begin{array}{l}\text { ANOVA } \\
P \text { value }\end{array}$ & $\begin{array}{c}\text { CircWave } \\
P \text { value }\end{array}$ & $\begin{array}{c}\mathrm{R}^{2} \text { (no } \\
\text { harmonics) }\end{array}$ & $\begin{array}{c}\mathrm{R}^{2} \text { (one } \\
\text { harmonic) }\end{array}$ & $\begin{array}{c}\text { F test } \\
\alpha\end{array}$ & $\begin{array}{c}\text { Average } \\
\text { concentration } \\
(\mathrm{ng} / \mathrm{ml})\end{array}$ & $\begin{array}{l}\text { Peak time } \\
\text { (h) }\end{array}$ & $\begin{array}{c}\text { Amplitude } \\
\text { ( } \% \text { of } \\
\text { mean) }\end{array}$ \\
\hline Visfatin & TSD & 0.020 & 0.005 & 0.054 & 0.103 & $>0.05$ & $0.64 \pm 0.03^{a}$ & $1337^{a}$ & 21.5 \\
\hline \multirow[t]{2}{*}{ Leptin } & Sleep & 0.764 & 0.086 & 0.027 & 0.043 & $>0.05$ & $3.15 \pm 0.10$ & 0204 & 9.2 \\
\hline & TSD & 0.574 & 0.035 & 0.031 & 0.056 & $>0.05$ & $3.17 \pm 0.10$ & 0143 & 9.0 \\
\hline
\end{tabular}

Serum concentrations of visfatin and leptin were measured during $24 \mathrm{~h}$ containing a regular sleep-wake cycle (sleep) and $24 \mathrm{~h}$ of continuous wakefulness (TSD), respectively. Circadian rhythmicity analysis was performed using CircWave (14), a modified Fourier method based on a combination of sine and cosine wave-fitting (equation: $f(t)=a+\sum\left(i=1\right.$ to $\infty\left[p_{i} \sin i(2 \pi t) / \tau+q_{i} \cos i(2 \pi t) / \tau\right]$ with $a=$ baseline; $i=$ number of harmonics; $p$ and $q=$ fit variables for sine and cosine parts of each harmonic; $t=$ time; $\tau=$ period, in this case 24 h) that allows for inclusion of harmonic oscillations to account for variations in curve shape. $\mathrm{F}$ tests were performed to assess the optimal complexity of the best-fit curve (i.e. the number of incorporated harmonic oscillations). The $\alpha$ cutoff was set to 0.05; all datasets showed best fits with a first-order sine/cosine fit, i.e. without harmonic oscillations. Phase differences in oscillations between sleep and TSD were assessed by comparing the peak phases for each curve fit. Peak time is shown according to the 24-h clock.

a $P<0.05$ for comparisons between conditions (Wilcoxon signed rank tests)

displayed a higher average concentration (both $P<0.05$, Wilcoxon signed rank test). Serum leptin concentrations peaked during the first half of the night in the sleep condition but subsequently failed to show a significant rhythm $(P<0.09$; Fig. 1B and Table 1$)$. In the TSD condition, the leptin rhythm reached significance $(P<0.04)$. However, this rhythm was not significantly different from that observed in the sleep condition $(P>0.43)$. Sleep times in the sleep condition were typical for laboratory conditions: total sleep, $418 \pm 8 \mathrm{~min}$; wake, $11 \pm 3 \mathrm{~min}$; stage 1 , $25 \pm 4 \mathrm{~min}$; stage 2, $242 \pm 8 \mathrm{~min}$; slow-wave sleep, $67 \pm$ $6 \mathrm{~min}$; rapid eye movement sleep, $72 \pm 6 \mathrm{~min}$; sleep onset latency, $32 \pm 8 \mathrm{~min}$; slow-wave sleep latency, $24 \pm 4 \mathrm{~min}$; and rapid eye movement sleep latency, $97 \pm 12 \mathrm{~min}$.

\section{Association between sleep-loss effects on postprandial glucose metabolism and on Nampt}

Before breakfast intake at $0730 \mathrm{~h}$, neither plasma glucose nor serum insulin concentrations differed between conditions (TSD $v$ s. sleep: plasma glucose, $5.0 \pm 0.1 v s$. $4.9 \pm 0.1 \mathrm{mmol} /$ liter; serum insulin, $300 \pm 33$ vs. $263 \pm$ $41 \mathrm{pmol} /$ liter; $P>0.38$ for all comparisons). Postprandial plasma glucose concentrations assessed $120 \mathrm{~min}$ after breakfast intake were increased after sleep loss $(6.1 \pm 0.2$ vs. $5.5 \pm 0.2 \mathrm{mmol} /$ liter, $P<0.04 ; P<0.03$ for TSD/ sleep $\times$ time interaction; Fig. $1 C)$. In contrast, postprandial insulin was comparable between conditions (451 \pm 45 vs. $429 \pm 45 \mathrm{pmol} /$ liter; $P>0.58 ; P=0.30$ for TSD/ sleep $\times$ time). Correlational analyses of individual differences between conditions (i.e. TSD minus sleep) revealed that the increase in postprandial plasma glucose concentration (expressed as percent relative to baseline) was positively associated with the magnitude of the sleep loss-induced phase shift in the Nampt rhythm (Spearman's $\rho=0.54 ; P<0.05$; Fig. 1D).

\section{Discussion}

We demonstrate in humans that the serum concentrations of Nampt follow a distinct diurnal rhythm, which peaks during the early afternoon. TSD in comparison with regular sleep phase advanced this rhythm and increased average Nampt concentrations, suggesting that sleep actively down-regulates circulating Nampt levels in humans. This conclusion is in accordance with recent cross-sectional examinations of more than 500 adults of the Cleveland Family Study showing that each hour of total sleep time reduction was associated with a $14 \%$ increase in serum Nampt concentrations (8). The Nampt rhythm per se was preserved when our subjects remained awake throughout the night. Although the present data do not allow a definite distinction between internal and external rhythms, this finding suggests that factors such as the circadian clock prevail in the rhythmic regulation of serum Nampt concentrations and possibly also of Nampt release (7). Compared with circulating Nampt concentrations that peaked during the wake phase (i.e. a period of high metabolic activity), serum leptin displayed an inverse pattern with peak concentrations during the inactive, nocturnal phase (i.e. a period characterized by a low resting metabolic rate), which is in line with previous observations (15). Leptin is well known to induce anorexigenic effects $(16,17)$, and preliminary findings suggest that Nampt/visfatin likewise affects food intake $(18,19)$. Thus, theinverse 24 -h patterns of Nampt and leptin, respectively, might bear some functional significance for the regulation of ingestive behavior. Unlike in previous studies $(11,15)$, sleep loss did not significantly affect leptin rhythms which most probably was due to the relatively low sample size of our study.

The phase shift in the 24-h Nampt rhythm induced by acute sleep deprivation was positively related to the sleep 
A

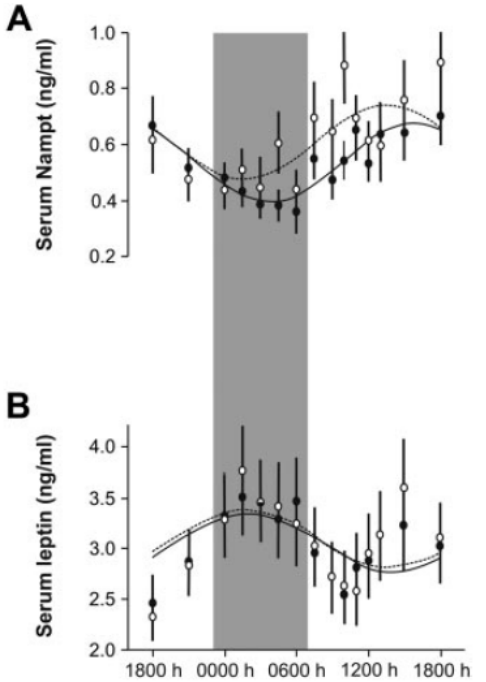

C
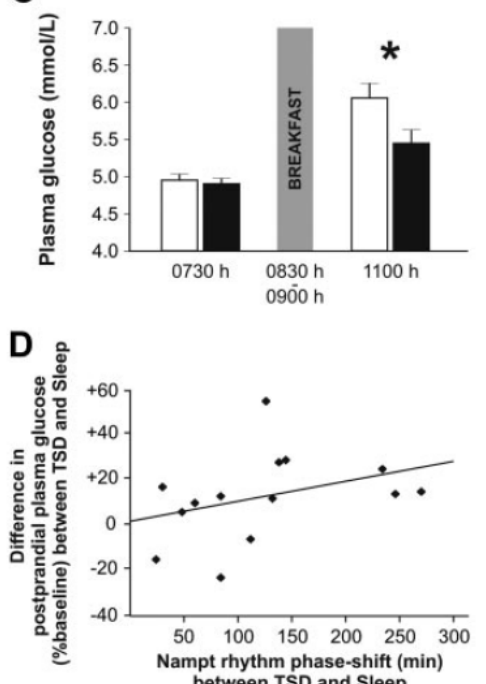

between TSD and Sleep

FIG. 1. Diurnal rhythms of Nampt and leptin and relation of Nampt to postprandial glucose. A and B, Average ( \pm SEM) levels and sine wave regressions of serum Nampt $(A)$ and serum leptin (B) concentrations measured in 14 healthy men during a regular 24-h sleep-wake cycle starting at $1800 \mathrm{~h}$ (sleep; filled circles, solid line) and during $24 \mathrm{~h}$ of continuous wakefulness (TSD; open circles, dashed line), respectively. Shaded area indicates nocturnal sleep in the sleep condition. Nampt concentrations showed a diurnal rhythm in both conditions (sleep, $P<0.001$; TSD, $P<0.005)$, with a significant phase advancement of about $2 \mathrm{~h}$ in the TSD compared with the sleep condition $(P<0.03)$ Leptin concentrations displayed a 24-h rhythm in the TSD $(P<0.04)$ but not in the sleep condition $(P<0.09)$ with no differences between conditions $(P>0.43)$. C, Plasma glucose concentrations before and after the ingestion of $3800 \mathrm{~kJ}$ of liquid food containing $112.8 \mathrm{~g}$ carbohydrate at $0830 \mathrm{~h}$ after nocturnal sleep (sleep; black bars) and wakefulness (TSD; white bars), respectively. ${ }^{*}, P<0.05$. D, Individual differences between conditions in postprandial plasma glucose levels (percent relative to baseline) plotted against individual magnitudes of the Nampt phase shift between conditions. All differences are TSD minus sleep. Spearman's $\rho=0.54 ; P<0.05$. loss-induced increase in 120-min postprandial plasma glucose concentrations assessed in the morning. This relationship indicates that the stronger the phase-shifting impact of nocturnal wakefulness on the regulation of Nampt levels, the more pronounced the impairing effect of sleep loss on glucose tolerance becomes. This pattern supports our hypothesis that the detrimental effect of compromised sleep on glucose homeostasis (9-11) might involve disturbances in the diurnal regulation of Nampt. Such an interpretation fits with previous experiments indicating that circulating concentrations of Nampt/visfatin are increased during acute hyperglycemia (20). Moreover, serum Nampt concentrations have been repeatedly shown to be elevated under conditions of chronically reduced glucose tolerance like type 2 diabetes $(2,3)$ and obesity $(4,5)$. Nevertheless, our results are of correlational nature, and there is good reason to assume that other factors such as the activation of neuroendocrine stress axes due to sleep deprivation $(9,11,12)$ may contribute to the effect of sleep restriction on glucose homeostasis.

In summary, we demonstrate that the circulating concentrations of Nampt follow a 24-h rhythm that peaks during the early afternoon. Sleep deprivation induces a significant rhythm phase shift in circulating Nampt levels that is directly related to the sleep loss-associated impairment of postprandial glucose metabolism. Future causeeffect experiments conducted under free-living conditions of unrestrained physical activity and also including acute and subchronic partial sleep loss interventions should reveal the potential ramifications of Nampt phase shifts for the regulation of glucose metabolism.

\section{Acknowledgments}

Address all correspondence and requests for reprints to: Christian Benedict, Department of Neuroscience, Uppsala University, Box 593, SE-751 24 Uppsala, Sweden. E-mail: christian.benedict@neuro.uu.se.

The study was funded by Deutsche Forschungsgemeinschaft (SFB 654, Emmy Noether Program), Bonn, Germany. Work from C.B., S.J.B., and H.B.S. is supported by the Swedish Research Council, Stockholm, Sweden. The funding sources had no input in the design and conduct of this study; in the collection, analysis, and interpretation of the data; or in the preparation, review, or approval of the manuscript.

The authors' responsibilities were as follows: C.B., T.L., J.B., B.S., and M.H. designed the study; C.B., A.S., H.O., T.L., and M.H. analyzed data; and all authors critically revised the manuscript for important intellectual content and contributed to writing the manuscript. All authors had full access to all of the data and take responsibility for the integrity and accuracy of the data analysis.

Disclosure Summary: None of the authors have conflicts of interest. 


\section{References}

1. Samal B, Sun Y, Stearns G, Xie C, Suggs S, McNiece I 1994 Cloning and characterization of the cDNA encoding a novel human pre-Bcell colony-enhancing factor. Mol Cell Biol 14:1431-1437

2. Chen MP, Chung FM, Chang DM, Tsai JC, Huang HF, Shin SJ, Lee YJ 2006 Elevated plasma level of visfatin/pre-B cell colony-enhancing factor in patients with type 2 diabetes mellitus. J Clin Endocrino Metab 91:295-299

3. Hammarstedt A, Pihlajamäki J, Rotter Sopasakis V, Gogg S, Jansson PA, Laakso M, Smith U 2006 Visfatin is an adipokine, but it is not regulated by thiazolidinediones. J Clin Endocrinol Metab 91:11811184

4. Hallschmid M, Randeva H, Tan BK, Kern W, Lehnert H 2009 Relationship between cerebrospinal fluid visfatin (PBEF/Nampt) levels and adiposity in humans. Diabetes 58:637-640

5. Berndt J, Klöting N, Kralisch S, Kovacs P, Fasshauer M, Schön MR, Stumvoll M, Blüher M 2005 Plasma visfatin concentrations and fat depot-specific mRNA expression in humans. Diabetes 54:29112916

6. Friebe D, Neef M, Kratzsch J, Erbs S, Dittrich K, Garten A, PetzoldQuinque S, Blüher S, Reinehr T, Stumvoll M, Blüher M, Kiess W, Körner A 2011 Leucocytes are a major source of circulating nicotinamide phosphoribosyltransferase (Nampt)/pre-B cell colony enhancing factor (PBEF) linking obesity and inflammation in humans. Diabetologia 54:1200-1211

7. Ramsey KM, Yoshino J, Brace CS, Abrassart D, Kobayashi Y, Marcheva B, Hong HK, Chong JL, Buhr ED, Lee C, Takahashi JS, Imai S, Bass J 2009 Circadian clock feedback cycle through NAMPT-mediated NAD ${ }^{+}$biosynthesis. Science 324:651-654

8. Hayes AL, Xu F, Babineau D, Patel SR 2011 Sleep duration and circulating adipokine levels. Sleep 34:147-152

9. Schmid SM, Hallschmid M, Jauch-Chara K, Wilms B, Lehnert H, Born J, Schultes B 2011 Disturbed glucoregulatory response to food intake after moderate sleep restriction. Sleep 34:371-377

10. Buxton OM, Pavlova M, Reid EW, Wang W, Simonson DC, Adler
GK 2010 Sleep restriction for 1 week reduces insulin sensitivity in healthy men. Diabetes 59:2126-2133

11. Spiegel K, Leproult R, L'hermite-Balériaux M, Copinschi G, Penev PD, Van Cauter E 2004 Leptin levels are dependent on sleep duration: relationships with sympathovagal balance, carbohydrate regulation, cortisol, and thyrotropin. J Clin Endocrinol Metab 89: $5762-5771$

12. Benedict C, Hallschmid M, Lassen A, Mahnke C, Schultes B, Schiöth HB, Born J, Lange T 2011 Acute sleep deprivation reduces energy expenditure in healthy men. Am J Clin Nutr 93:1229-1236

13. Rechtschaffen A, Kales A 1968 A manual of standardized terminology, techniques and scoring system for sleep of human subjects. Washington, DC: U.S. Government Printing Office

14. Oster H, Damerow S, Hut RA, Eichele G 2006 Transcriptional profiling in the adrenal gland reveals circadian regulation of hormone biosynthesis genes and nucleosome assembly genes. J Biol Rhythms 21:350-361

15. Heptulla R, Smitten A, Teague B, Tamborlane WV, Ma YZ, Caprio S 2001 Temporal patterns of circulating leptin levels in lean and obese adolescents: relationships to insulin, growth hormone, and free fatty acids rhythmicity. J Clin Endocrinol Metab 86:90-96

16. Halaas JL, Gajiwala KS, Maffei M, Cohen SL, Chait BT, Rabinowitz D, Lallone RL, Burley SK, Friedman JM 1995 Weight-reducing effects of the plasma protein encoded by the obese gene. Science 269:543-546

17. Morton GJ, Cummings DE, Baskin DG, Barsh GS, Schwartz MW 2006 Central nervous system control of food intake and body weight. Nature 443:289-295

18. Cline MA, Nandar W, Prall BC, Bowden CN, Denbow DM 2008 Central visfatin causes orexigenic effects in chicks. Behav Brain Res 186:293-297

19. Park BS, Jin SH, Park JJ, Park JW, Namgoong IS, Kim YI, Lee BJ, Kim JG 2011 Visfatin induces sickness responses in the brain. PLoS One 6:e15981

20. Haider DG, Schaller G, Kapiotis S, Maier C, Luger A, Wolzt M 2006 The release of the adipocytokine visfatin is regulated by glucose and insulin. Diabetologia 49:1909-1914 
Publication: Synaptotagmin10-Cre, a driver to disrupt clock genes in the SCN

Jana Husse, Xunlei Zhou, Anton Shostak, Henrik Oster and Gregor Eichele

Journal of Biological Rhythms, Volume 26, pages 379-389

Anton Shostak performed behavioral characterization of WT, Syt $10^{\mathrm{Cre} /+} \mathrm{Bmal}^{\mathrm{flfl} I}$, Syt $10^{\mathrm{Cre} /+} \mathrm{Bmal}^{\mathrm{fl}-}$ and Syt10 ${ }^{\text {Cre/Cre }} \mathrm{Bmal}^{\mathrm{fl} /-}$ animals as shown in Figure 5. 


\title{
Synaptotagmin10-Cre, a Driver to Disrupt Clock Genes in the SCN
}

\author{
Jana Husse, Xunlei Zhou, ${ }^{1}$ Anton Shostak, Henrik Oster, and Gregor Eichele ${ }^{2}$ \\ Genes and Behavior Department, Max Planck Institute for Biophysical Chemistry, \\ Goettingen, Germany
}

\begin{abstract}
Surgical lesion of the suprachiasmatic nuclei $(\mathrm{SCN})$ profoundly affects the circadian timing system. A complication of SCN ablations is the concomitant scission of SCN afferents and efferents. Genetic disruption of the molecular clockwork in the SCN provides a complementary, less invasive experimental approach. The authors report the generation and functional analysis of a new Cre recombinase driver mouse that evokes homologous recombination with high efficiency in the SCN. They inserted the Cre recombinase cDNA into the Synaptotagmin10 (Syt10) locus, a gene strongly expressed in the SCN. Heterozygous Synaptotagmin10-Cre $\left(\right.$ Syt10 $\left.{ }^{\mathrm{Cr}}\right)$ mice have no obvious circadian locomotor phenotype, and homozygous animals show slightly reduced light-induced phase delays. Crosses of $S y t 10^{\mathrm{Cre}}$ mice with $\beta$-galactosidase reporter animals revealed strong Cre activity in the vast majority of SCN cells. Cre activity is not detected in nonneuronal tissues with the exception of the testis. The authors demonstrate that conditionally deleting the clock gene Bmal1 using the Syt10 ${ }^{\text {Cre }}$ driver renders animals arrhythmic.
\end{abstract}

Key words suprachiasmatic nucleus, SCN, circadian, clock, Bmal1, Cre driver, Synaptotagmin10

In the mouse, gene targeting in embryonic stem (ES) cells as well as $N$-ethyl-N-nitrosourea $(E N U)$ mediated mutagenesis plays a key role in elucidating the molecular basis of the mammalian circadian clock (Bunger et al., 2000; van der Horst et al., 1999; Vitaterna et al., 1994; Zheng et al., 2001; Zheng et al., 1999). The mammalian circadian clockwork rests on a limited number of core clock proteins that form interlocked transcriptional-translational feedback loops creating a 24-h rhythm. This machinery drives rhythmic expression of hundreds of clock-controlled genes that regulate a wide range of rhythmic physiological functions (Ko and Takahashi, 2006). Clocks have been found in almost all cells and tissues of the body. They are equipped with the complete molecular clockwork and exhibit a self-sustained circadian rhythm even when kept in explant culture (Balsalobre et al., 1998; Tosini and Menaker, 1996; Yamazaki et al., 2000; Yoo et al., 2004). The mammalian circadian system is organized in a hierarchical manner with the central pacemaker in the suprachiasmatic nucleus $(\mathrm{SCN})$ controlling numerous peripheral clocks (Dibner et al., 2010). Pioneering lesion studies carried out in the early 1970 s established the SCN as the central

1. Present address: University of Heidelberg, Institute of Anatomy and Cell Biology, Heidelberg, Germany.

2. To whom all correspondence should be addressed: Gregor Eichele, Genes and Behavior Department, Max Planck Institute for Biophysical Chemistry, Am Fassberg 11, 37077 Goettingen, Germany; e-mail: Gregor.Eichele@mpibpc.mpg.de.

JOURNAL OF BIOLOGICAL RHYTHMS, Vol. 26 No, 5, October 2011 379-389

DOI: $10.1177 / 0748730411415363$

(C) 2011 The Author(s) 
pacemaker (Moore and Eichler 1972; Stephan and Zucker 1972).

Over the past decade, mice deficient for each of the core clock genes have been generated. Typically, such animals are characterized by smaller or greater defects in locomotor circadian rhythmicity and, additionally, may also show a number of phenotypes, including metabolic defects, changes in the reward system, or memory impairments (Abarca et al., 2002; Garcia et al., 2000; Turek et al., 2005). It is as yet unclear whether these defects are clock mediated and, if they are, which tissue clocks exert control. In the adrenal, for example, transplantation experiments have been used to address the function of a tissue clock (Kiessling et al., 2010; Oster et al., 2006). An alternative to transplantation and surgical ablation is the tissue-specific deletion of core clock genes using the Cre-loxP system (Storch et al., 2007; Lamia et al., 2008; Marcheva et al., 2010).

In view of the overarching role of the $\mathrm{SCN}$ in circadian timekeeping, we attempted to knock-out clock genes such as Bmal1 specifically in this nucleus. To achieve this goal, we generated a SCN Cre driver that can delete conditional alleles of genes of interest in this nucleus. The Allen Brain Atlas (Lein et al., 2007) has uncovered numerous genes that are expressed in the SCN. However, genes solely expressed therein were not found. In situ hybridization on brain sections identified Synaptotagmin10 (Syt10) as a gene that is strongly expressed in the $\mathrm{SCN}$ with relatively few other expression sites in the CNS, even during development (www.genepaint.org, Genepaint ID MH808). Synaptotagmins are involved in regulated exocytosis of synaptic vesicles and are thought to function as calcium sensors (Gustavsson and Han, 2009). Most Synaptotagmins are widely expressed throughout the nervous system, and Syt1, 2, 4, 5, 9, 11, 13, 14, and 16 are also found in the $\mathrm{SCN}$, albeit much less enriched therein than Syt10. The presence of multiple Synaptotagmins in the $\mathrm{SCN}$ is beneficial for the design of a Cre driver mouse, as it makes it less likely that inserting the Cre recombinase gene into the Syt 10 locus would result in a major impairment of neuronal function as paralogs of Syt10 should compensate for a loss of this particular Synaptotagmin.

Here we report the generation and characterization of a Syt $10^{\text {cre }}$ driver line that enables SCN targeting without targeting of peripheral, nonneuronal clocks. We verified the usefulness of the $S y t 10^{\mathrm{Cre}}$ driver line by knocking out a conditional Bmal1 allele. Depending on the dosage of $\mathrm{Cre}$ recombinase, we obtained mice with phenotypes ranging from minimal circadian perturbation to complete arrhythmicity.

\section{MATERIALS AND METHODS}

\author{
Cloning of Syt10 ${ }^{\mathrm{Cre}}$ Targeting Vector
}

We replaced the ATG in exon 1 of the Syt10 gene by a Cre cassette. Nts 92-229 (NM_018803.2) were replaced with the Cre cassette composed of the $i \mathrm{Cre}$ (Shimshek et al., 2002), an internal ribosomal entry site (IRES), followed by an enhanced green fluorescent protein (EGFP) reporter cDNA and a Flippase recognition target (FRT)-flanked PGKneomycin selection marker. An $8.7-\mathrm{kb}$ genomic region ( $5.3 \mathrm{~kb}$ upstream of exon 1 and $3.4 \mathrm{~kb}$ downstream of exon 1) was cloned by recombineering (Liu et al., 2003) from a BAC clone (BMQ295d20). Exon 1 was then replaced by the Cre knock-in cassette.

\section{Generation and Genotyping of Syt $10^{\text {Cre }}$ Knock-in Mice}

Gene targeting of Syt10 ${ }^{\text {cre }}$ knock-in mice was performed by GenOway using 129Sv/Pas embryonic stem cells. DNA from G418-resistant clones was digested with SphI and analyzed by Southern blotting. Targeted cells were identified using a 474-bp probe generated by PCR (P1; forward primer: 5'-CAAGATGGCT TCTTTAATGACCCCAG-3', reverse primer: 5'-AGAGT TGCACACACTTCGGTGCAC-3') that hybridizes with the 3' homology arm. SphI digestion of the targeted allele resulted in an 8.9-kb band in addition to the 14.7-kb wild-type band (Fig. 1A). For positive clones, the $5^{\prime}$ homology arm was independently tested using a suitable PCR probe. Targeted clones were injected into blastocysts. Chimeric offspring were backcrossed to C57BL/6. The resulting F1 generation was screened for germline transmission by Southern blotting as described for ES cells and PCR genotyping using the following primers: Syt10 F: 5'-AGACCTGGCAGCAGCGTCCGTTGG-3', Syt10 $R: 5^{\prime}$-AAGATAAGCTCCAGCCAGGAAGTC-3', and Syt10 KI R: 5'-GGCGAGGCAGG CCAGATCTCCTGTG-3'. PCR was performed for 38 cycles with an annealing temperature of $65^{\circ} \mathrm{C}$. A wild-type band of $426 \mathrm{bp}$ and a mutant band of 538 bp were separated on a $1.5 \%$ agarose gel (Fig. 1C). In order to delete the FRT-flanked neomycin cassette, mutant mice were crossed to an ubiquitously expressing Flippase line on C57BL/6 background (Farley et al., 2000). Offspring were tested for neomycin deletion by PCR. The Flippase allele was out-crossed in the next generation by back-crossing to C57BL/6. 


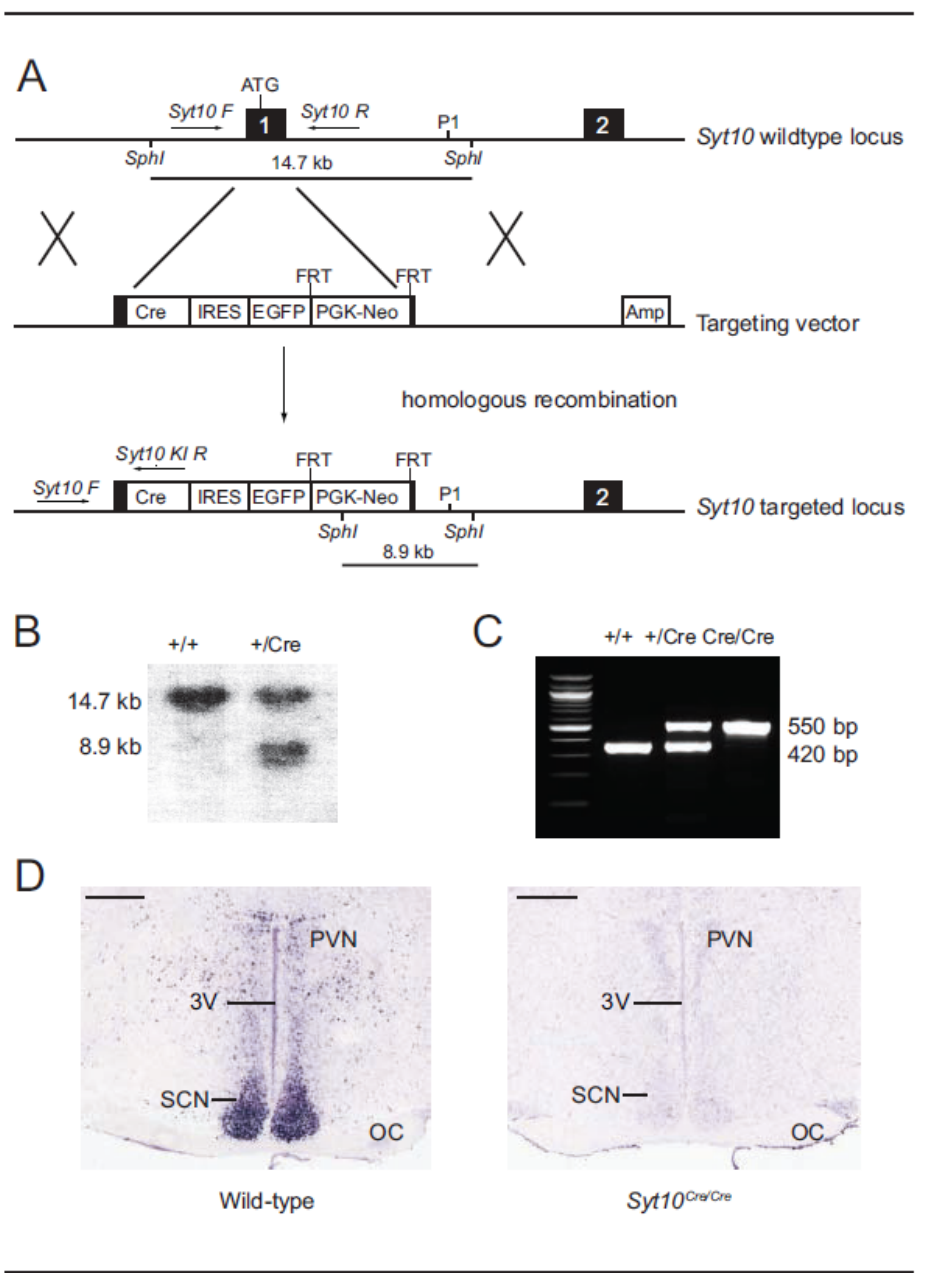

Figure 1. Generation of $S y t 10^{\text {Cre }}$ knock-in mice. (A) Schematic of the Syt10 wild-type locus, the targeting vector, and the resulting targeted locus. Exons are indicated in black. Position of genotyping primers ( $S y t 10$ F, Syt10 R, and Syt10 KI R) are depicted by arrows. Southern fragments are shown as lines. The probe used for Southern genotyping is marked as P1. (B) Southern genotyping of F1 littermates obtained from chimera-C57BL/6 crosses. A 474-bp probe (P1) detected a $14.7-\mathrm{kb}$ wild-type and an 8.9-kb mutant SphI fragment. (C) PCR genotyping of F1 littermates obtained from Syt10 ${ }^{\mathrm{Crel} / \mathrm{t}}$ intercrosses using the genotyping primers Syt10 F, Syt10 R, and Syt10 KI R. A 420-bp wild-type and a 550-bp mutant band are separated on a $1.5 \%$ agarose gel. (D) Syt10 expression in the SCN as determined by in situ hybridization in wild-type and Syt10 ${ }^{\text {CrelCre }}$ mice. Scale bar: $0.5 \mathrm{~mm}$. Abbreviations: $3 \mathrm{~V}$, third ventricle; Amp, ampicillin resistance gene; Cre, Cre recombinase; FRT, Flippase recognition target; EGFP, enhanced green fluorescent protein; IRES, internal ribosomal entry site; Neo, neomycin resistance; OC, optic chiasm; PGK, phosphoglycerine kinase A promoter; PVN, paraventricular nucleus of the hypothalamus; SCN, suprachiasmatic nucleus.

\section{$\beta$-Galactosidase Staining}

Syt $10^{\text {Cre/ } /+} R 26 R^{\text {LacZ/ } / \text { mice were sacrificed by cervical }}$ dislocation; brains were quickly removed and frozen in O.C.T (Tissue-Tek). Then, $25-\mu \mathrm{m}$ cryosections were stained for $\beta$-galactosidase as described (Sakurai et al.,
2005) and counterstained with Nuclear Fast Red or DAPI. Quantification of recombination efficiency was performed as follows: $10-\mu \mathrm{m}$ cryosections were first stained with DAPI followed by colorimetric detection of $\beta$-galactosidase activity. The SCN was outlined and the number of DAPI-stained nuclei (a measure of cell number) and of $\beta$-galactosidase dots were counted, and the ratio of $\beta$-galactosidase dots/nuclei was computed. Two sections per $\mathrm{SCN}$ region (rostral, central, caudal) from 4 different animals were included. An identical analysis was carried out on SCNs of animals in which $\beta$-galactosidase activity is seen throughout the SCN (see Suppl. Table S1 for further information). Finally, the ratio of these two counts was calculated to obtain the percentage of SCN cells expressing Cre recombinase in Syt $10^{\text {Cre/+ }}$ R26R LacZ/+ $^{\text {mice. }}$

\section{In Situ Hybridization}

Automated in situ hybridization on $25-\mu \mathrm{m}$ frozen sections was performed as described (Visel et al., 2007; Yaylaoglu et al., 2005). A 1019-bp Syt10 template was used for riboprobe generation (NM_018803,nts 279 to 1298). Riboprobe concentration was $200 \mathrm{ng} / \mu \mathrm{L}$.

Gene Expression Analysis by Reverse Transcription PCR

Syt $10^{\mathrm{Cre} / \mathrm{t}}$ males were sacrificed and tissues were harvested in RNA Later solution (Ambion). RNA was Trizolextracted (Invitrogen) and DNAse treated (TURBO DNA-free Kit, Ambion). CDNA synthesis was performed (Superscript II, Invitrogen) with the Oligo-dT primer. A 490-bp PCR fragment encompassing the Cre sequence was amplified using the following primers: $\mathrm{Cre}$ RT forward: 5'-GTGGATGCTGGGGAGAGAGCCAAGC-3' and Cre RT reverse: 5'-CAGACCAGGCCAGGTATCTCTGCCC-3'. From the same samples, Eefla1 was amplified as an internal standard. 
Gene Expression Analysis by Quantitative Real-Time PCR

Animals were sacrificed at zeitgeber time (ZT) 18, brains were harvested, and brain punches of the anterior ventral hypothalamus comprising the $\mathrm{SCN}$ were taken from 1-mm-thick brain sections. RNA and cDNA were prepared as described above. QPCR was performed using iQ SYBR Green Supermix on an iCycler thermocycler (Bio-Rad) according to the manufacturer's protocol. Eefla1 was used as a standard, and quantification was performed as described (Kiessling et al., 2010). Primer sequences were as follows: Bmal1 forward: 5'-TGACCCTCATGGAAGGTTAGAA-3', Bmal1 reverse: 5'-CAGCCATCCTTAGCACGGT-3', Eefla1 forward: 5'-AATTCACCAACACCAGCAGCAA-3', and Eefla1 reverse: 5'-TGCCCCAGGACACAGAGACTTCA-3'. Sample sizes were 9 animals for wild-type and 3 animals for each of the other genotypes.

\section{Immunohistochemistry}

First, $10-\mu \mathrm{m}$ frozen sections were fixed in ice-cold $4 \%$ PFA for $15 \mathrm{~min}$, washed 3 times in TNT buffer ( 10 $\mathrm{mM}$ Tris- $\mathrm{HCl}, 150 \mathrm{mM} \mathrm{NaCl}$, and $0.05 \%$ Tween), blocked in $10 \%$ normal goat serum for $1 \mathrm{~h}$, and incubated with anti-BMAL1 antibody (1:1000 rabbit anti-MOP3; Novus Biologicals) overnight at $4{ }^{\circ} \mathrm{C}$. The next day, sections were washed 3 times with TNT buffer, incubated with the secondary antibody (1:400 anti-rabbit AF488; Invitrogen) for $2 \mathrm{~h}$ at room temperature, washed again, and mounted with DAPI containing mounting medium. BMAL1-positive cells in a $240 \times 187 \mu \mathrm{m}$ rectangle in 3 medial SCN sections were counted. For each genotype, 3 animals were analyzed.

\section{Behavioral Experiments}

All animal experiments were carried out in compliance with the German Law on Animal Welfare. Breeding strategies can be found in Supplementary Table S1. Mouse housing and behavioral monitoring were performed as described (Jud et al., 2005). Males on a mixed 129Sv/C57BL/ 6 background of 2 to 5 months of age were used. Controls: where feasible, littermates were used. Mice were kept on a 12:12 light dark (LD, 350-lux) cycle before transfer to constant darkness (DD) or constant light (LL, 100 lux). A 15-min 350-lux light pulse was given manually at circadian time (CT) 14. Behavioral data were analyzed using
ClockLab acquisition and analysis software package (Actimetrics). Period and amplitude were calculated using $\chi^{2}$ periodogram analysis. Phase shifts in an Aschoff type I protocol were calculated as described (Jud et al., 2005). Onset error was calculated as mean deviation of real onset from a least squares-fit regression line over a period of 10 consecutive days in DD. Ultradian (period of 5-10 h) amplitudes were calculated using $\chi^{2}$ periodogram analysis of 5 consecutive days of the second week in DD.

\section{Data Analysis}

Statistical comparisons were made in GraphPad Prism, and $p$ values below 0.05 were considered significant. Normality tests revealed that not all behavioral data followed a Gaussian distribution, and hence nonparametric analyses were performed throughout: Mann-Whitney $U$ test for comparison of two groups and Kruskal-Wallis test for comparison of morethantwogroups.QPCRandimmunohistochemistry data were analyzed using $t$ tests.

\section{RESULTS}

\section{Generation of $S y t 10^{c r e}$ Mice}

The construct used for targeting the Syt10 locus replaced the endogenous Syt10 ATG located in exon 1 by the Cre cDNA sequence (Fig. 1A). This targeting should result in a knock-out of endogenous Syt10. We generated Syt $10^{\mathrm{Cre}}$ mice by $129 \mathrm{~Sv}$ ES cell targeting and subsequent blastocyst injections. We verified the genotype of F1 animals by Southern blotting and PCR (Fig. 1B,C). Subsequently, the FRT-flanked neomycin resistance cassette was deleted. The deletion of Syt10 was confirmed by in situ hybridization to coronal SCN sections. Syt10 transcripts were not detectable in homozygous Syt $10^{\mathrm{Cr} / / \mathrm{Cre}}$ mutants, neither in the SCN (Fig. 1D) nor in any of the other Syt10 expression sites in the brain (not shown).

Syt $10^{\text {Cre }}$ heterozygous and homozygous mice are viable and fertile with no obvious morphological abnormalities. Breeding experiments revealed that $\mathrm{Cre}$ is active in the male germline. Hence, offspring of a father who is Syt $10^{\text {cre }}$ positive and also carries a conditional allele of the gene to be deleted will be completely deficient in the paternally derived allele of the gene of interest. In the case of the reporter $R 26 R L a c Z$, the resulting embryos will stain for $\beta$-galactosidase ubiquitously (for further information, see Suppl. Table S1). 


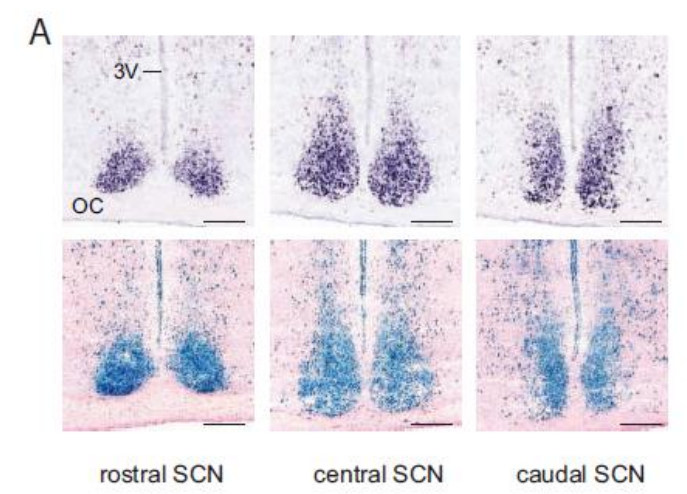

B

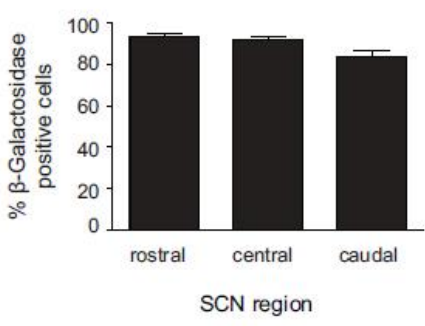

Figure 2. Strong Cre activity in the SCN of Syt10 ${ }^{\text {crel+ }} R 26 R^{\text {LacZ }+}$ mice. (A) Syt10 expression and Cre activity at different levels along the rostrocaudal axis of the $\mathrm{SCN}$ as determined by in situ hybridization (upper panels) or $\beta$-galactosidase staining (lower panels). Sections shown for the two techniques are $25 \mu \mathrm{m}$ apart. Scale bar: $0.2 \mathrm{~mm}$. (B) Percentage of $\beta$-galactosidase positive cells in the $\mathrm{SCN}$. No significant differences were detected between the $3 \mathrm{SCN}$ axial levels $(n=4)$. Abbreviations: $3 \mathrm{~V}$, third ventricle; $\mathrm{OC}$, optic chiasm.

\section{Activity of Cre Is Brain Specific} and SCN Enriched

We crossed Syt $10^{\mathrm{Cre}}$ mice to the R26RLacZ reporter line that expresses $\beta$-galactosidase after $C$ re-mediated excision of a stop cassette (Soriano, 1999). Consistent with the in situ hybridization data, $\beta$-galactosidase staining was very strong throughout the $\mathrm{SCN}$ (Fig. 2A). To estimate the percentage of $\mathrm{SCN}$ cells expressing $\mathrm{Cre}$ recombinase, the number of $\beta$-galactosidase dots in the SCN of $S y t 10^{\mathrm{Cr} / /+} \mathrm{R} 26 R^{\text {Lacz/t+ }}$ mice was determined relative to the $\mathrm{SCN}$ of mice that ubiquitously express $\beta$-galactosidase (see Materials and Method). We found that $\sim 90 \%$ of the SCN cells in Syt10 ${ }^{\mathrm{Cre} / \mathrm{t}} \mathrm{R} 26 \mathrm{R}^{\mathrm{LacZ} / \mathrm{t}}$ mice were $\beta$-galactosidase positive (Fig. 2B). The variation between rostral, central, and caudal levels $(93 \%, 92 \%$, and $84 \%$ respectively) is not significant. The $\beta$-galactosidase negative cells could be glia cells that do not express Syt10 (Zhang et al., 2004).

Cre activity was detected in a variety of other brain structures. Coronal sections at the level of the SCN showing Syt10 expression detected by in situ hybridization (Suppl. Fig. S1A) and Cre activity detected by $\beta$-galactosidase reporter analysis (Suppl. Fig. S1B) give a good indication of the extent to which Cre is active in non-SCN areas. Supplementary Figure S2 shows $\beta$-galactosidase staining in a variety of brain tissues. We observed that Cre-expressing cells are embedded in numerous non-Cre-expressing cells. By contrast, in the SCN, the majority of cells are $\beta$-galactosidase positive (Fig. 2A). The $\beta$-galactosidase reporter expression pattern was also seen using the human alkaline phosphatase reporter line (Lobe et al., 1999). Phosphatase staining pattern in these mice was very similar to that of $\beta$-galactosidase (data not shown).

To examine whether Cre-induced recombination occurred outside the CNS, weperformed $\beta$-galactosidase staining on sections of various tissues isolated from Syt $10^{\text {Crel+ }}$ R26RLacZ ${ }^{\text {LacZ/+ }}$ mice (Fig. 3A). We did not detect any Cre activity in peripheral tissues except for the seminiferous tubules of the testis, an expression site presaged by the breeding experiments (Suppl. Table S1). To further confirm the lack of Cre expression in peripheral tissues, we performed $\mathrm{Cre}$-specific reverse transcription PCR reactions on RNA isolated from 19 tissues of Syt10 $\mathrm{Cre} /+$ mice. A Cre PCR product was detected only in neuronal tissues, including the eye and the spinal cord, and in testis (Fig. 3B). In summary, the Syt $10^{\text {Cre }}$ driver is highly active in the SCN and, to a somewhat lesser extent, in other brain regions. Importantly, we did not detect any Cre activity in nonneuronal tissues with the exception of testis.

\section{A Minor Light-Resetting Phenotype in Syt10 Crel/Cre Homozygous Mice}

We analyzed the circadian behavior of heterozygous and homozygous Syt10 ${ }^{\mathrm{Cre}}$ mice. Male wild-type, $S y t 10^{\mathrm{Cre} /+}$ and Syt $10^{\mathrm{Cre} / \mathrm{Cre}}$ littermates were tested for wheel-running behavior in a standard experimental setup. In a 12:12 LD cycle, Syt10 ${ }^{\mathrm{Cre} / \mathrm{t}}$ as well as Syt $10^{\mathrm{Cre} / \mathrm{Cre}}$ mice entrained normally (Fig. 4A). Total activity levels as well as onset variability were not different between genotypes. When released into DD, all genotypes showed a similar endogenous period (Fig. 4B; $23.5 \mathrm{~h}, 23.4 \mathrm{~h}$, and $23.7 \mathrm{~h}$ in wild-type, Syt $10^{\mathrm{Cre} / \mathrm{t}}$, and $S y t 10^{\mathrm{Cr} / \mathrm{Cre} e}$, respectively). Locomotor activity onset error as a measure of rhythm instability was also not different between genotypes (Fig. 4C). 


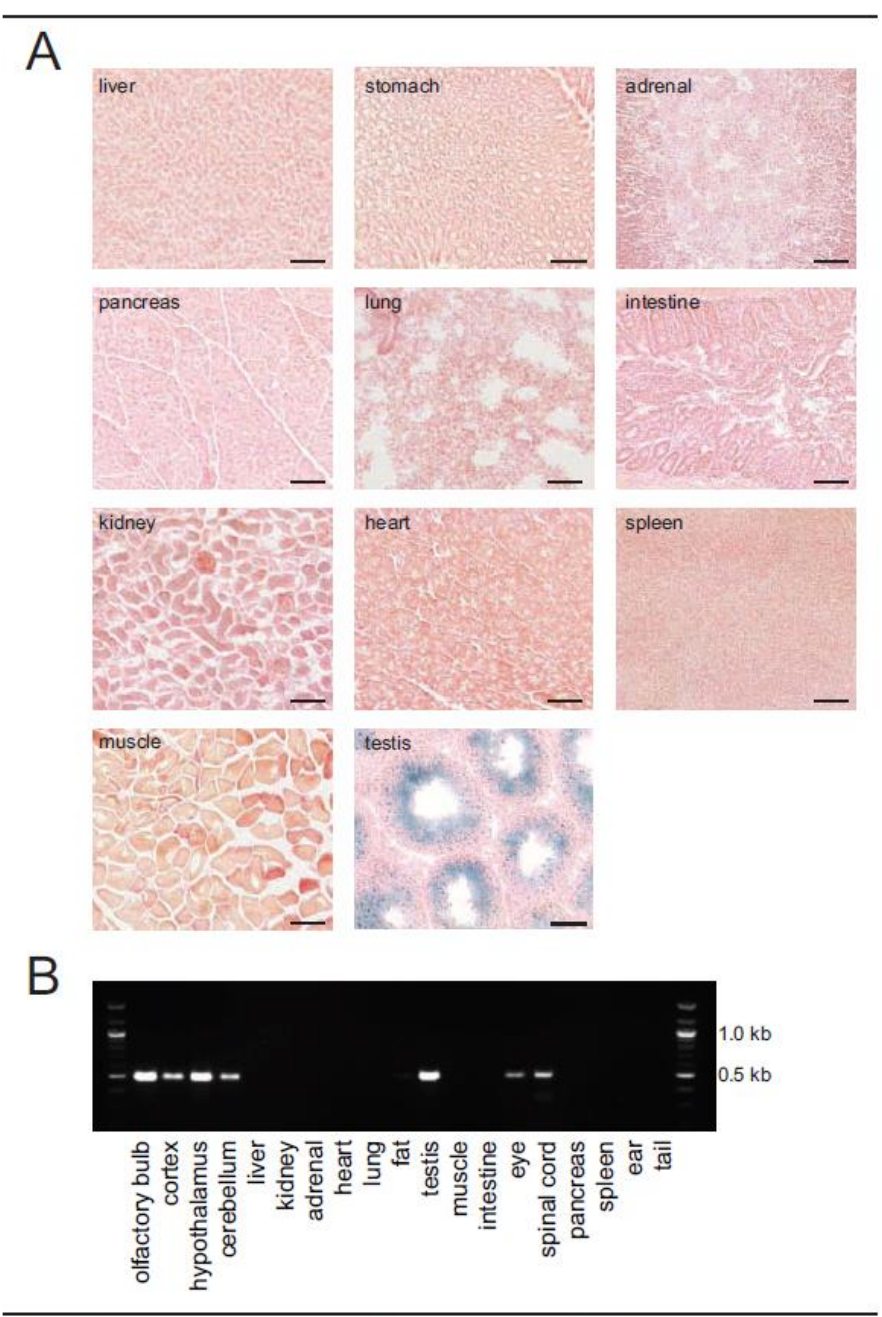

Figure 3. Cre activity is detected only in neuronal tissues and in testis. (A) $\beta$-galactosidase staining of various organs isolated from Syt10 ${ }^{\mathrm{Crel}+} \mathrm{R} 26 \mathrm{RLacZ} \mathrm{Z}^{+-}$mice No Cre-mediated recombination resulting in $\beta$-galactosidase expression is detectable in peripheral organs with the exception of the seminiferous tubules of the testis. Scale bar: $0.1 \mathrm{~mm}$. (B) Cre-specific reverse transcription PCRs on RNA isolated from various tissues of $\mathrm{Sy} t 10^{\mathrm{Crel}+}$ mice.

Next, we investigated the light response of the circadian system in all 3 genotypes by giving a 15-min light pulse at CT14. Wild-type as well as heterozygous mice showed the expected phase delay of approximately $140 \mathrm{~min}$ (Benloucif and Dubocovich, 1996). Syt10 cre/cre mice, however, displayed a reduced phase delay of 90 $\min$ (Fig. 4D; wild-type vs. Syt10 ${ }^{\text {cre/cre }} ; p=0.006$ ). Given this slightly reduced light response of the circadian system in homozygous mice, we determined their freerunning period in LL. This parameter is influenced by both the endogenous circadian period and the light responsiveness of the circadian system (Daan and Pittendrigh, 1976). We did not find differences in the period between wild-type and heterozygous littermates. Homozygous mutants, however, showed a slightly shortened period in LL (Fig. 4E; $25.1 \mathrm{~h}$ and $24.6 \mathrm{~h}$ in wild-type and Syt $10^{\mathrm{Cr} / \mathrm{Cr} \text {, }}$, respectively; $p=$ $0.03)$.

Therefore, the Syt $10^{\text {cre }}$ driver line even in the homozygous state seems suitable for circadian experiments as it shows only a minor light-resetting phenotype. Both the circadian period and the stability of locomotor activity rhythms under LD and DD conditions are normal. Heterozygous mutants do not show any impairment in the tested circadian behavioral paradigms.

Cre Dosage-Dependent Circadian Phenotype in Conditional Bmal1-Deficient Mice

The only single-gene knock-out identified so far that produces a complete arrhythmic locomotor phenotype is Bmal1 (Bunger et al., 2000). Hence, we crossed the $\operatorname{Syt} 10^{\mathrm{Cre}}$ line with a mouse line carrying a conditional allele of Bmal1 that allows Cre-mediated deletion of the exon encoding the BMAL1 basic helix loophelix (bHLH) domain (Bmal1 ${ }^{\text {P/f }}$ ) (Storchetal., 2007). We analyzed wheel-running behavior in 12:12 LD and DD conditions.

Syt10 ${ }^{\text {Cre/+ }}$ Bmal1 $1 / f l$ mice showed a shortened period in DD (Fig. 5A,B; $23.5 \mathrm{~h}$ and $22.9 \mathrm{~h}$ in wild-type and Syt $10^{\mathrm{Cr} / \mathrm{t}}$ Bmal1 $^{f / / f}$, respectively; $p=0.002$ ). These mice were still rhythmic under both LD and DD conditions (Fig. 5A and Suppl. Fig. S3). However, rhythmicity in DD was less stable, and quantification of the onset error revealed increased onset variability in Syt $10^{\mathrm{Cre} / \mathrm{H}} \mathrm{Bmal1}^{\mathrm{A} / \mathrm{f}}$ mice compared to wild-type controls (Fig. 5C; $0.38 \mathrm{~h}$ and $0.78 \mathrm{~h}$ in wildtype and Syt $10^{\mathrm{Cre} / \mathrm{S}}$ Bmal1 $^{\mathrm{Al} / \mathrm{f}}$, respectively; $p=0.038$ ). It appears that in Syt $10^{\mathrm{Cr} / \mathrm{H}} \mathrm{Bmal1} / \mathrm{f/fl}$ mice, the amount of Cre activity was insufficient to fully delete Bmal1 in all $\mathrm{SCN}$ cells.

Next we used a Bmal1 $1^{\mathrm{A} /}$ - background that, based on previous work, should in itself not produce a circadian 


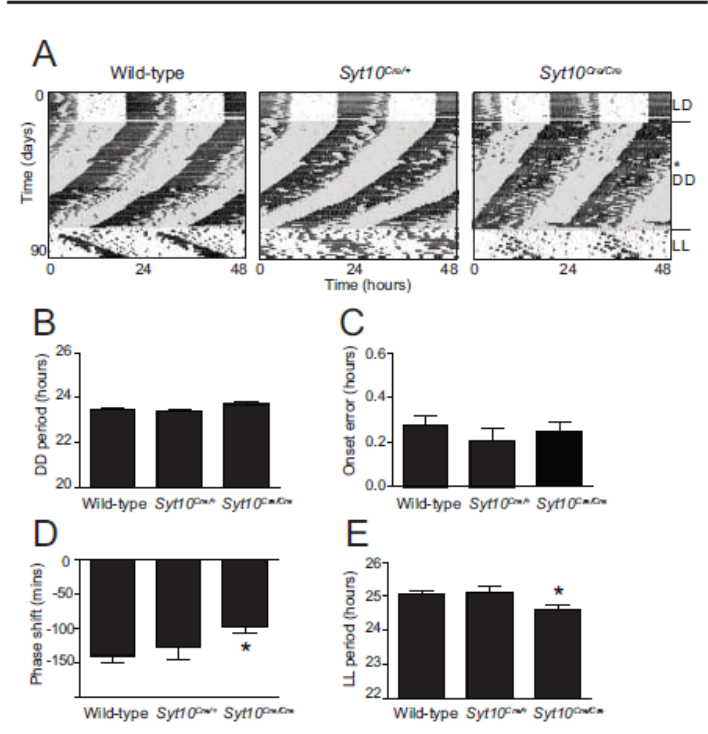

Figure 4. No major circadian locomotor impairments in Syt10 mice. (A) Representative double-plotted actograms of wild-type, Syt10 ${ }^{\mathrm{Crel}+}$, and Syt10 $\mathrm{Cre/Cre}$ mice kept in a 12-h light/12-h dark cycle (LD) and sequentially released in constant darkness (DD) followed by constant light (LL). The day of a 15-min light pulse is indicated with a star. (B) Magnitude of period in constant darkness by $\chi^{2}$ periodogram analysis. (C) Onset error in constant darkness. (D) Magnitude of phase shift after a 15-min light pulse at CT14. (E) Magnitude of period in constant light by $\chi^{2}$ periodogram analysis. All data are shown as mean and SEM and analyzed using a MannWhitney test, ${ }^{*}$ indicates $p<0.05$ tested against wild-type, $n=8$ per genotype.

phenotype (Bunger et al., 2000; Storch et al., 2007) but would clearly require less Cre activity since one Bmal1 allele is already mutated. Such $\mathrm{Sy}_{\mathrm{t}} 10^{\mathrm{Cr} / \mathrm{L}} \mathrm{Bmal}^{\mathrm{fl} /}$ animals have a shorter period in DD compared to wild-type (Fig. 5A,B, Suppl. Fig. S3; $23.5 \mathrm{~h}$ and $22.6 \mathrm{~h}$ in wild-type and Syt10 ${ }^{\mathrm{Cre} / \mathrm{H}} \mathrm{Bmal1}^{\mathrm{fl} / \text {, }}$, respectively; $\left.p=0.0004\right)$. Onset variability analysis revealed that $\mathrm{Syt} 10^{\mathrm{Cr} / \mathrm{H}} \mathrm{Bmal} 1^{\mathrm{f} / \mathrm{L}}$ animals have higher onset errors than wild-type controls (Fig. 5C; $0.38 \mathrm{~h}$ and $1.31 \mathrm{~h}$ in wild-type and Syt $10^{\mathrm{Cre} / \mathrm{H}} \mathrm{Bmal}^{\mathrm{fl} /}$, respectively; $p=0.0024)$. Overall, $S y t 10^{\mathrm{Cr} / \mathrm{H}} \mathrm{Bmal}^{\mathrm{fl} /}$ animals display impaired activity rhythms but are not completely arrhythmic. This led us to conclude that a single Cre allele is insufficient to produce enough Bmal1-deficient cells in the SCN to result in total circadian arrhythmicity.

We next examined Syt $10^{\mathrm{Cr} / \mathrm{Cre}}$ Bmal1 $\mathrm{fl}^{\mathrm{f} / \mathrm{m}}$ mice and did get a fully arrhythmic phenotype (Fig. 5A and Suppl. Fig. S3). Ten of 11 animals were totally arrhythmic in DD; a $\chi^{2}$ periodogram analysis did not reveal any significant circadian rhythmicity. In LD conditions, these mice were still rhythmic; however, in the light phase, they were more active than wild-type controls
(5.3\% and $18.2 \%$ light activity in wild-type and Syt $10^{\text {cre/Cre }}$ Bmal1 $^{f / /}$, respectively; $p=0.046$ ). One best appreciates the extent of the arrhythmic phenotype of $S y t 10^{\mathrm{Cr} / \mathrm{Cre}}$ Bmal1 $^{\mathrm{fl} /-}$ mice by a comparison with pan-Bmal1 knockout animals (Syt10 $\mathrm{Cre}^{\mathrm{Cre}} \mathrm{Bmal1}^{-/}$). The phenotype of Syt $10^{\mathrm{Cr} / \mathrm{Cre}} \mathrm{Bmal}^{\mathrm{Al} /}$ is indistinguishable from the Syt $10^{\mathrm{Cr} / \mathrm{Cre}}$ Bmal1 ${ }^{--}$phenotype in LD and in DD (Fig. 5 and Suppl. Fig. S3). Periodograms of both genotypes show no clear circadian peak (Fig. 5A). Thus, the Syt $10^{\mathrm{Cr} / / \mathrm{Cre}} \mathrm{Bmal1}^{\mathrm{fl} /-}$ model is a very efficient $\mathrm{SCN}$ knock-out and completely mimics the circadian behavioral phenotype of panBmal1 knock-out mice. Overall, the onset variability, a measure for rhythm instability, was progressively increasing with increasing likelihood that both Bmal1 alleles were deleted (Fig. 5C). It has been shown before that in arrhythmic clock mutants, ultradian rhythms can become more prominent (Abraham et al., 2006). We therefore analyzed the periodogram amplitude in the ultradian range $(5-10 \mathrm{~h})$ in the different genotypes. Decreasing circadian amplitudes (Fig. 5A) clearly correlated with increasing ultradian amplitudes (Fig. 5D). In summary, the Syt $10^{\text {Cre }}$ evokes a variety of circadian phenotypes ranging from normal rhythmicity to totally arrhythmic phenotypes in a Cre dosage-dependent manner. Increasing the probability of a recombined Bmal1 allele by either using 2 Cre alleles or by working with a $f l$ - background increases the severity of the phenotype, eventually leading to a complete loss of circadian locomotor rhythmicity in Syt $10^{\mathrm{Cre} / \mathrm{Cre}}$ Bmal1 ${ }^{f / /}$ mice.

To examine whether the Cre dose-dependent circadian defects correlate with the amount of Bmal1 expression in the $\mathrm{SCN}$, we quantified Bmal1 mRNA and BMAL1 protein levels in wild-type, Syt1 ${ }^{\mathrm{Cre} / \mathrm{B}} \mathrm{Bmal1}^{\mathrm{fl} / \text {, }}$ and Syt $10^{\mathrm{Cre} / \mathrm{Cre}} \mathrm{Bmal1}^{\mathrm{Al} /}$ mice at ZT18, which is the time point of maximal Bmal1 expression in the SCN (Oishi et al., 2000). QPCR analysis of SCN punches revealed a significant reduction of Bmal1 mRNA levels to less than $50 \%$ of wild-type levels in both conditional genotypes (Fig. $6 \mathrm{~A} ; 47 \%$ and $40 \%$ in Syt $10^{\mathrm{Cre} / \mathrm{H}} \mathrm{Bmal1}^{\mathrm{fl} / \mathrm{-}}$ and Syt $10^{\mathrm{Cre} / \mathrm{t}}$ ${ }^{C r e} B_{m a l 1}{ }^{f /-} ; t$ test; $p=0.0081$ and $p=0.004$, respectively). Differences between Syt $10^{\mathrm{Crel+}} \mathrm{Bmal} 1^{\mathrm{fl} / \mathrm{L}}$ and Syt $10^{\mathrm{Cr} / \mathrm{Cre}}$ $B$ mal1 ${ }^{\mathrm{A} /}$ genotypes were not significant. This analysis may underestimate the degree of knock-out in the SCN since SCN punches contain non-SCN tissue in which Bmal1 expression is normal. We thus performed immunohistochemistry with an anti-BMAL1 antiserum (for validation of the anti-BMAL1 immunoreactivity, see Suppl. Fig. S4). A progressive reduction of BMAL1 immunoreactivity with increasing Cre dosage was seen (Fig. 6B). Relative to wild-type, the SCN of Syt $10^{\mathrm{Cre} / \mathrm{t}}$ Bmal1 $^{\text {f/- }}$ mice had a reduction of BMAL1-positive cells 


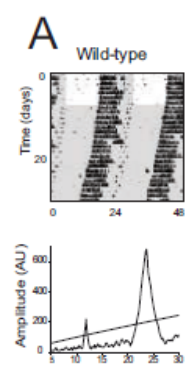

B

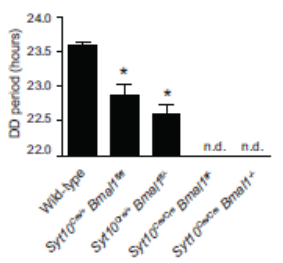

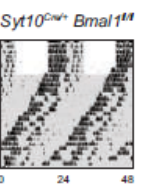

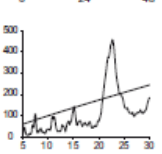

C
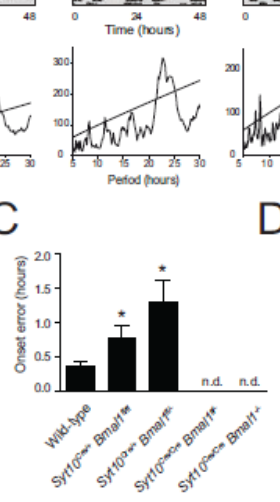
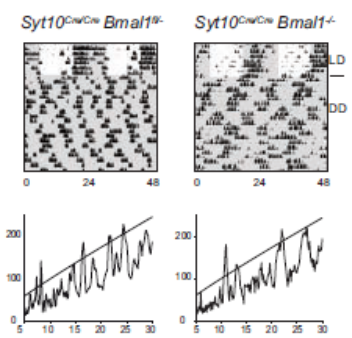

$\mathrm{D}$

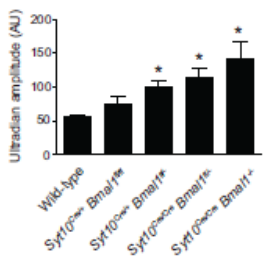

Figure 5. Circadian phenotypes in Syt10 ${ }^{\text {Cre }}$-driven Bmal1 knock-outs. (A) Representative double-plotted actograms and periodograms of wild-type, Syt10 Crel+

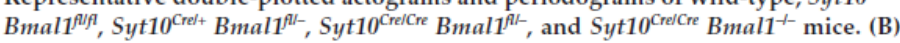
Magnitude of period in DD determined by $\chi^{2}$ periodogram analysis. In $S y t 10^{\mathrm{Cre} / \mathrm{Cr} e}$ Bmal1 $^{f l-}$ and Syt10 ${ }^{\mathrm{Cr} / \mathrm{Cr} \text { B }}$ Bmal1 ${ }^{-1-}$ mice, no circadian rhythmicity was detected (n.d.). (C) Onset error was determined for consecutive 10 days in DD. (D) Amplitudes in the ultradian range $(5-10 \mathrm{~h})$ were calculated from 5 days in DD. All data are shown as mean and SEM and analyzed using a Mann-Whitney test; ${ }^{*}$ indicates $p<0.05$ tested against wild-type.

to $35 \%$, and in the SCN of Syt $10^{\mathrm{Cr} / / \mathrm{Cre}}$ Bmal1 $1^{\mathrm{fl}-}$ animals, merely $17 \%$ of the cells expressed BMAL1 (Fig. 6C; $t$ test; $p=0.0012$ and $p=0.0005$, respectively, tested against wild-type). The number of BMAL1-positive cells in Syt $10^{\mathrm{Cr} / \mathrm{Cre}}$ Bmalf $^{\text {I/ }}$ animals was significantly reduced compared to Syt10 $\mathrm{Cre}^{\mathrm{t}} \mathrm{Bmal1}^{\mathrm{fl} / \mathrm{-}}$ animals (Fig. 6C; $t$ test; $p=0.0077$ ). The expression of BMAL1 thus correlated with the behavioral phenotypes of these conditional mutants (see Fig. 5).

\section{DISCUSSION}

The Syt $10^{\text {Cre }}$ driver mouse line will be useful to delete conditional alleles of clock or other genes that are expressed in the SCN. Cre activity is found only in neuronal tissues and seminiferous tubules of the testis. Cre activity is strong in the vast majority of SCN cells. We demonstrate that knocking out a conditional allele of the essential clock gene Bmal1 using the Syt $10^{\mathrm{Cre}}$ driver renders animals arrhythmic as expected from an efficient SCN clock knock-out. Additionally, we show that the number of BMAL1-positive cells in the SCN correlates with the severity of the behavioral phenotype.
A significant benefit of a knock-in strategy is that it avoids typical problems seen with transgenics such as positional or copy number effects that might complicate an analysis of Cre-mediated effects. By expression of $\mathrm{Cre}$ from the Syt10 locus, we achieve Cre activity patterns that are highly similar to the endogenous Syt10 expression pattern. The driver is capable of targeting the majority of SCN cells. The cells that show no Cre-mediated $\beta$-galactosidase signal could be glial cells that are present in the SCN (Van den Pol, 1980) and do not to express Syt10 (Zhang et al., 2004).

Knocking out Syt10 has no major effect on circadian behavior, as the period in constant darkness and the entrainment to light-dark cycles remain unchanged. There is, however, a one-third reduction in the magnitude of light-induced phase delays and a slight decrease in the period in constant light. The fact that circadian impairments in $S y t 10^{\mathrm{Cre}}$ mice are minor may be due to the expression of several other Synaptotagmins in the SCN. The lack of a pronounced circadian phenotype in Syt10 Cre mice is a prerequisite for making the $S y t 10^{\text {cre }}$ driver line suitable for circadian research.

We observed drastic effects on circadian rhythmicity when the Syt $10^{\text {Cre }}$ driver line is used to delete Bmal1. This result is in line with transplantation experiments and inducible expression of dominant negative CLOCK protein, both of which show that the genotype of the SCN determines locomotor period (Hong et al., 2007; Ko et al., 2010; Ralph et al., 1990; Sujino et al., 2003). Germline deletion of Bmal1 and restoring Bmal1 expression under the Secretogranin 2 promoter in the brain causes not only circadian defects but also a reduction in life span, body weight, and overall activity levels (Bunger et al., 2005; Bunger et al., 2000; McDearmon et al., 2006). Such deficiencies are not seen in Syt $10^{\mathrm{Cre} / \mathrm{Cre}} \mathrm{Bmal1}^{\mathrm{Hl} / \mathrm{a}}$ animals, an advantage that will facilitate further analysis of the circadian phenotype of these mice. Lesion and transplantation experiments led to one of the major advances in chronobiological research, the discovery of the $\mathrm{SCN}$ as the master pacemaker of the mammalian brain (Moore and Eichler, 1972; Ralph et al., 1990; Stephan and Zucker, 1972). The chief difference between deleting Bmal1 in the SCN and SCN lesion experiments is that in the former case, the SCN afferent and efferent neuronal connections are not impaired. The fact that SCN 


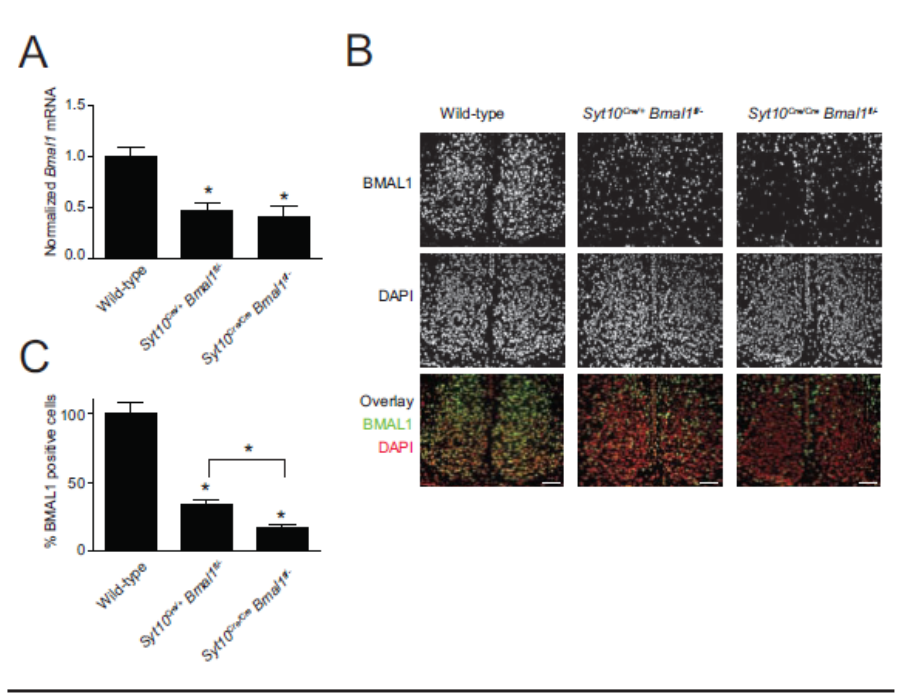

Figure 6. Quantification of Bmal1/BMAL1 levels in the SCN. (A) Relative expression levels of Bmal1 in SCN punches. (B) Representative images of SCN sections immunostained with anti-BMAL1 antibody (top row) and counterstained with DAPI (center). The lower row shows the overlay of BMAL1 immunoreactivity (green) and DAPI (red). Scale bar: $75 \mu \mathrm{m}$. (C) Percentage of BMAL1-positive cells in mutant SCN relative to wild-type. All data are shown as mean and SEM and analyzed using a $t$ test; ${ }^{*}$ indicates $p<0.05$.

afferents and efferents are destroyed in such lesion experiments may complicate experiments investigating the role of neuronal connections between the $\mathrm{SCN}$ and the periphery.

We found gene dosage effects in that the number of BMAL1-positive cells in the SCN correlated with behavioral rhythmicity. This is reminiscent of what has been shown for the CLOCK mutation. It was found that the ratio of CLOCK mutant and wild-type cells in the SCN correlated with behavioral rhythmicity (LowZeddies and Takahashi, 2001). The ability to evoke gene deletion in a graded manner should be informative when examining how clock gene dosage in the SCN affects circadian rhythmicity or the expression of other (clock) genes. The benefit of such titrating experiments has also been demonstrated in a recent elegant in vitro study (Baggs et al., 2009). These authors downregulated clock genes in a dose-dependent manner by adding different amounts of small interfering RNAs to human osteosarcoma cells. This led them to uncover novel network features of the circadian timing system. In the context of a recent study showing stochastic rhythmicity in complete Bmal1 knock-out mice (Ko et al., 2010), it will be interesting to investigate whether and how a network. stepwise reduction of Bmal1 in the SCN affects oscillations of SCN neuronal networks.

The currently known canonical clock genes are not required for viability. However, it would not come as a surprise if yet unknown clock genes existed that are also required for a range of noncircadian functions, including viability. Potential examples are Creb or Glycogen synthase kinase-3 3 (litaka et al., 2005; Obrietan et al., 1999). In such cases, the $S y t 10^{\text {cre }}$ driver mouse can be useful to overcome lethality and produce mice suitable for circadian analysis.

When using the Syt $10^{\mathrm{Cr}}$ driver line, one should consider the following points: First, the efficiency of Cre-mediated homologous recombination might vary depending on the targeted allele used. It has been shown that the exact location and distance between two loxP sites can affect recombination efficacy (Zheng et al., 2000). Thus, deletion of other conditional gene alleles might require less $\mathrm{Cre}$ to elicit a full penetrance of phenotype than in the case of Bmal1 $1^{\mathrm{f} / \mathrm{fl}}$. Hence, Syt $10^{\mathrm{Cre} / \mathrm{t}}$ and Syt $10^{\mathrm{Cr} / \mathrm{Cre}}$ should be compared with respect to their recombination efficiency. Second, only females (and not males) that are carriers for Syt $10^{\mathrm{Cre}}$ and the conditional allele of interest must be used for breeding. Otherwise, germline deletion of the conditional allele will occur, and all cells in the body will be deficient for the gene of interest. A guide for breeding strategies using the Syt10 $0^{\text {re }}$ is found in Supplementary Table S1. A caveat with using Syt $10^{C r e}$ in combination with a mouse in which one of the targeted alleles has already been removed (e.g., Bmal $1^{f / L}$ ) is that the heterozygous targeted animal may already have a phenotype. In summary, we believe that our Syt $10^{\mathrm{Cr}}$ line will be a helpful tool to investigate the complexity of the mammalian circadian

\section{ACKNOWLEDGMENTS}

We thank Axel Visel and Christina Thaller for their early work on expression patterns of Synaptotagmins. We thank Johanna Barclay for helpful comments on the manuscript. H.O. is an Emmy Noether Fellow of the German Research Foundation (DFG). 


\section{CONFLICT OF INTEREST STATEMENT}

The author(s) have no potential conflicts of interest with respect to the research, authorship, and/or publication of this article.

\section{NOTE}

Supplementary online material for this article is available on the Journal of Biological Rhythms website at http://jbr.sagepub.com/supplemental.

\section{REFERENCES}

Abarca C, Albrecht U, and Spanagel R (2002) Cocaine sensitization and reward are under the influence of circadian genes and rhythm. Proc Natl Acad Sci U S A 99:9026-9030.

Abraham D, Dallmann R, Steinlechner S, Albrecht U, Eichele G, and Oster H (2006) Restoration of circadian rhythmicity in circadian clock-deficient mice in constant light. J Biol Rhythms 21:169-176.

Baggs JE, Price TS, DiTacchio L, Panda S, Fitzgerald GA, and Hogenesch JB (2009) Network features of the mammalian circadian clock. PLoS Biol 7:e52.

Balsalobre A, Damiola F, and Schibler U (1998) A serum shock induces circadian gene expression in mammalian tissue culture cells. Cell 93:929-937.

Benloucif S and Dubocovich ML (1996) Melatonin and light induce phase shifts of circadian activity rhythms in the C3H/HeN mouse. J Biol Rhythms 11:113-125.

Bunger MK, Walisser JA, Sullivan R, Manley PA, Moran SM, Kalscheur VL, Colman RJ, and Bradfield CA (2005) Progressive arthropathy in mice with a targeted disruption of the Mop3/Bmal-1 locus. Genesis 41:122-132.

Bunger MK, Wilsbacher LD, Moran SM, Clendenin C, Radcliffe LA, Hogenesch JB, Simon MC, Takahashi JS, and Bradfield CA (2000) Mop3 is an essential component of the master circadian pacemaker in mammals. Cell 103:1009-1017.

Daan S and Pittendrigh CS (1976) A functional analysis of circadian pacemakers in nocturnal rodents. J Comp Physiol A Neuroethol Sens Neural Behav Physiol 106:253-266.

Dibner C, Schibler U, and Albrecht U (2010) The mammalian circadian timing system: Organization and coordination of central and peripheral clocks. Annu Rev Physiol 72:517-549.

Farley FW, Soriano P, Steffen LS, and Dymecki SM (2000) Widespread recombinase expression using FLPeR (flipper) mice. Genesis 28:106-110.

Garcia JA, Zhang D, Estill SJ, Michnoff C, Rutter J, Reick M, Scott K, Diaz-Arrastia R, and McKnight SL (2000) Impaired cued and contextual memory in NPAS2deficient mice. Science 288:2226-2230.

Gustavsson N and Han W (2009) Calcium-sensing beyond neurotransmitters: Functions of synaptotagmins in neuroendocrine and endocrine secretion. Biosci Rep 29:245-259.

Hong HK, Chong JL, Song W, Song EJ, Jyawook AA, Schook AC, Ko CH, and Takahashi JS (2007) Inducible and reversible Clock gene expression in brain using the tTA system for the study of circadian behavior. PLoS Genet 3:e33.

Iitaka C, Miyazaki K, Akaike T, and Ishida N (2005) A role for glycogen synthase kinase-3beta in the mammalian circadian clock. J Biol Chem 280:29397-29402.

Jud C, Schmutz I, Hampp G, Oster H, and Albrecht U (2005) A guideline for analyzing circadian wheel-running behavior in rodents under different lighting conditions. Biol Proced Online 7:101-116.

Kiessling S, Eichele G, and Oster H (2010) Adrenal glucocorticoids have a key role in circadian resynchronization in a mouse model of jet lag. J Clin Invest 120: 2600-2609.

Ko CH and Takahashi JS (2006) Molecular components of the mammalian circadian clock. Hum Mol Genet 15(Suppl 2):R271-R277.

Ko CH, Yamada YR, Welsh DK, Buhr ED, Liu AC, Zhang EE, Ralph MR, Kay SA, Forger DB, and Takahashi JS (2010) Emergence of noise-induced oscillations in the central circadian pacemaker. PLoS Biol 8:e1000513.

Lamia KA, Storch KF, and Weitz CJ (2008) Physiological significance of a peripheral tissue circadian clock. Proc Natl Acad Sci U S A 105:15172-15177.

Lein ES, Hawrylycz MJ, Ao N, Ayres M, Bensinger A, Bernard A, Boe AF, Boguski MS, Brockway KS, Byrnes EJ, et al. (2007) Genome-wide atlas of gene expression in the adult mouse brain. Nature 445:168-176.

Liu P, Jenkins NA, and Copeland NG (2003) A highly efficient recombineering-based method for generating conditional knockout mutations. Genome Res 13:476-484.

Lobe CG, Koop KE, Kreppner W, Lomeli H, Gertsenstein M, and Nagy A (1999) Z/AP, a double reporter for cremediated recombination. Dev Biol 208:281-292.

Low-Zeddies SS and Takahashi JS (2001) Chimera analysis of the Clock mutation in mice shows that complex cellular integration determines circadian behavior. Cell 105:25-42.

Marcheva B, Ramsey KM, Buhr ED, Kobayashi Y, Su H, Ko CH, Ivanova G, Omura C, Mo S, Vitaterna MH, et al. (2010) Disruption of the clock components CLOCK and BMAL1 leads to hypoinsulinaemia and diabetes. Nature 466:627-631.

McDearmon EL, Patel KN, Ko CH, Walisser JA, Schook AC, Chong J, Wilsbacher LD, Song EJ, Hong HK, Bradfield CA, et al. (2006) Dissecting the functions of the mammalian clock protein BMAL1 by tissue-specific rescue in mice. Science 314:1304-1308.

Moore RY and Eichler VB (1972) Loss of a circadian adrenal corticosterone rhythm following suprachiasmatic lesions in the rat. Brain Res 42:201-206.

Obrietan K, Impey S, Smith D, Athos J, and Storm DR (1999) Circadian regulation of cAMP response elementmediated gene expression in the suprachiasmatic nuclei. J Biol Chem 274:17748-17756.

Oishi K, Fukui H, and Ishida N (2000) Rhythmic expression of BMAL1 mRNA is altered in Clock mutant mice: 
Differential regulation in the suprachiasmatic nucleus and peripheral tissues. Biochem Biophys Res Commun 268:164-171.

Oster H, Damerow S, Kiessling S, Jakubcakova V, Abraham D, Tian J, Hoffmann MW, and Eichele G (2006) The circadian rhythm of glucocorticoids is regulated by a gating mechanism residing in the adrenal cortical clock. Cell Metab 4:163-173.

Ralph MR, Foster RG, Davis FC, and Menaker M (1990) Transplanted suprachiasmatic nucleus determines circadian period. Science 247:975-978.

Sakurai Y, Ohgimoto K, Kataoka Y, Yoshida N, and Shibuya M (2005) Essential role of Flk-1 (VEGF receptor 2) tyrosine residue 1173 in vasculogenesis in mice. Proc Natl Acad Sci US A 102:1076-1081.

Shimshek DR, Kim J, Hubner MR, Spergel DJ, Buchholz F, Casanova E, Stewart AF, Seeburg PH, and Sprengel R (2002) Codon-improved Cre recombinase (iCre) expression in the mouse. Genesis 32:19-26.

Soriano P (1999) Generalized lacZ expression with the ROSA26 Cre reporter strain. Nat Genet 21:70-71.

Stephan FK and Zucker I (1972) Circadian rhythms in drinking behavior and locomotor activity of rats are eliminated by hypothalamic lesions. Proc Natl Acad Sci U S A 69:1583-1586.

Storch KF, Paz C, Signorovitch J, Raviola E, Pawlyk B, Li T, and Weitz CJ (2007) Intrinsic circadian clock of the mammalian retina: Importance for retinal processing of visual information. Cell 130:730-741.

Sujino M, Masumoto KH, Yamaguchi S, van der Horst GT, Okamura H, and Inouye ST (2003) Suprachiasmatic nucleus grafts restore circadian behavioral rhythms of genetically arrhythmic mice. Curr Biol 13:664-668.

Tosini G and Menaker M (1996) Circadian rhythms in cultured mammalian retina. Science 272:419-421.

Turek FW, Joshu C, Kohsaka A, Lin E, Ivanova G, McDearmon E, Laposky A, Losee-Olson S, Easton A, Jensen DR, et al. (2005) Obesity and metabolic syndrome in circadian Clock mutant mice. Science 308:1043-1045.

Van den Pol AN (1980) The hypothalamic suprachiasmatic nucleus of rat: intrinsic anatomy. J Comp Neurol 191:661-702.

van der Horst GT, Muijtjens M, Kobayashi K, Takano R, Kanno S, Takao M, de Wit J, Verkerk A, Eker AP, van Leenen D, et al. (1999) Mammalian Cry1 and Cry2 are essential for maintenance of circadian rhythms. Nature 398:627-630.

Visel A, Carson J, Oldekamp J, Warnecke M, Jakubcakova V, Zhou X, Shaw CA, Alvarez-Bolado G, and Eichele G (2007) Regulatory pathway analysis by high-throughput in situ hybridization. PLoS Genet 3:1867-1883.

Vitaterna MH, King DP, Chang AM, Kornhauser JM, Lowrey PL, McDonald JD, Dove WF, Pinto LH, Turek FW, and Takahashi JS (1994) Mutagenesis and mapping of a mouse gene, Clock, essential for circadian behavior. Science 264:719-725.

Yamazaki S, Numano R, Abe M, Hida A, Takahashi R, Ueda M, Block GD, Sakaki Y, Menaker M, and Tei H (2000) Resetting central and peripheral circadian oscillators in transgenic rats. Science 288:682-685.

Yaylaoglu MB, Titmus A, Visel A, Alvarez-Bolado G, Thaller C, and Eichele G (2005) Comprehensive expression atlas of fibroblast growth factors and their receptors generated by a novel robotic in situ hybridization platform. Dev Dyn 234:371-386.

Yoo SH, Yamazaki S, Lowrey PL, Shimomura K, Ko CH, Buhr ED, Siepka SM, Hong HK, Oh WJ, Yoo OJ, et al. (2004) PERIOD2::LUCIFERASE real-time reporting of circadian dynamics reveals persistent circadian oscillations in mouse peripheral tissues. Proc Natl Acad Sci U S A 101:5339-5346.

Zhang Q, Fukuda M, Van Bockstaele E, Pascual O, and Haydon PG (2004) Synaptotagmin IV regulates glial glutamate release. Proc Natl Acad Sci U S A 101: 9441-9446.

Zheng B, Albrecht U, Kaasik K, Sage M, Lu W, Vaishnav S, Li Q, Sun ZS, Eichele G, Bradley A, et al. (2001) Nonredundant roles of the mPer1 and mPer2 genes in the mammalian circadian clock. Cell 105: 683-694.

Zheng B, Larkin DW, Albrecht U, Sun ZS, Sage M, Eichele G, Lee CC, and Bradley A (1999) The mPer2 gene encodes a functional component of the mammalian circadian clock. Nature 400:169-173.

Zheng B, Sage M, Sheppeard EA, Jurecic V, and Bradley A (2000) Engineering mouse chromosomes with Cre-loxP: Range, efficiency, and somatic applications. Mol Cell Biol 20:648-655. 


\section{Publication: Clock genes and sleep}

Landgraf $D^{*}$, Shostak A*, Oster H.

*-equal contribution

Pflügers Archiv - European Journal of Physiology Volume 463, pages 3-14

Anton Shostak contributed chapters:

introduction;

interaction between circadian and homeostatic sleep components;

clock genes and sleep timing;

clock genes and sleep homeostasis;

table 1

and revised the whole manuscript. 
Pflugers Arch - Eur J Physiol (2012) 463:3-14

DOI $10.1007 / \mathrm{s} 00424-011-1003-9$

INVITED REVIEW

\section{Clock genes and sleep}

Dominic Landgraf • Anton Shostak $\cdot$ Henrik Oster

Received: 21 February 2011 / Revised: 8 July 2011 /Accepted: 22 July 2011 / Published online: 11 August 2011

(C) Springer-Verlag 2011

Abstract In most species - from cyanobacteria to humansendogenous clocks have evolved that drive 24-h rhythms of behavior and physiology. In mammals, these circadian rhythms are regulated by a hierarchical network of cellular oscillators controlled by a set of clock genes organized in a system of interlocked transcriptional feedback loops. One of the most prominent outputs of the circadian system is the synchronization of the sleep-wake cycle with external (day-) time. Clock genes also have a strong impact on many other biological functions, such as memory formation, energy metabolism, and immunity. Remarkably, large overlaps exist between clock gene and sleep (loss) mediated effects on these processes. This review summarizes sleep clock gene interactions for these three phenomena, highlighting potential mediators linking sleep and/or clock function to physiological output in an attempt to better understand the complexity of diurnal adaptation and its consequences for health and disease.

Keywords Circadian clock · Clock genes · Sleep · Metabolism $\cdot$ Immunity $\cdot$ Memory

\section{Introduction}

Almost 40 years have passed since the first clock gene, period, was discovered by Ronald Konopka and Seymour

D. Landgraf and A. Shostak contributed equally to this work. This article is published as part of the Special Issue on Sleep.

D. Landgraf $\cdot$ A. Shostak $\cdot$ H. Oster $(\bowtie)$

Circadian Rhythms Group,

Max Planck Institute for Biophysical Chemistry,

Am Faßberg 11,

37077 Göttingen, Germany

e-mail: henrik.oster@mpibpc.mpg.de
Benzer in a forward genetic screen on fruit flies [75] Starting from this landmark finding, $N$-ethyl- $N$-nitrosourea (ENU) mutagenesis phenotypic screens became a powerful tool to unravel the genetic basis of circadian rhythms. In the mid-1990s, the first mammalian clock gene, circadian locomotor output cycles kaput (Clock), was identified and cloned by Martha Vitaterna in the lab of Joseph Takahashi [71, 156]. In mammals - as in most organisms studied so far-circadian rhythms are controlled by a set of clock genes forming a network of positive and negative autoregulatory feedback loops $[57,120]$. These clock genes are expressed in most tissues. A circadian pacemaker located in the suprachiasmatic nuclei $(\mathrm{SCN})$ of the hypothalamus is reset by external light stimuli and synchronizes peripheral oscillators throughout the body with each other and with the light-dark cycle via humoral, neuronal, and behavioral cues [120]. At its heart, the cellular circadian clockwork consists of a main (or core) and an auxiliary (or accessory) transcriptional-translational feedback loop (TTL; Fig. 1, left side). The former is composed of the positive components brain and muscle ARNT-like 1 (BMAL1 or ARNTL), CLOCK and neuronal PAS domain protein 2 (NPAS2), as well as the negative components CRYPTOCHROME $1 / 2$ (CRY1/2) and PERIOD 1-3 (PER1-3). BMAL1, CLOCK, and NPAS2 are members of the basic helix-loop-helix (bHLH) Per-Arnt-Sim (PAS) family of transcription factors. In the SCN, CLOCK/NPAS2 and BMAL1 form heterodimers that bind to specific circadian E-box elements on the promoters of their targets, thereby activating Cry and Per transcription during the (subjective) day. In the late afternoon, PER and CRY protein levels reach a critical concentration and, now forming complexes themselves, translocate into the nucleus. There, they interact with CLOCK/NPAS2-BMAL1 and, by repressing the transcription of their own genes, form a negative feedback loop. Progressive degradation of negative 


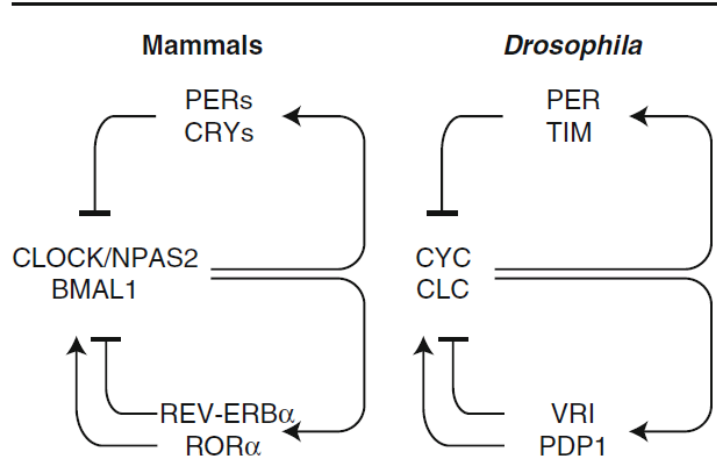

Fig. 1 Transcriptional-translational feedback loops regulate cellular circadian rhythms in mammals and flies. Both vertebrate and invertebrate clockworks are based on similar mechanistic concepts and share a number of genetic components. In mammals (left), a core loop is composed of PER and CRY proteins that inhibit their own transcription by inhibition of CLOCK (NPAS2)/BMAL1. An accessory loop involves REV-ERB $\alpha$ and $\operatorname{ROR} \alpha$ that regulate Bmall transcriptional rhythms. In Drosophila (right), CLC/CYC activate PER/TIM that feedback on CLC/CYC activity. VRI and PDP1 form an accessory loop that regulates $\mathrm{Clc}$ transcriptional rhythms

regulators towards the end of the subjective night starts a new cycle by the re-activation of Per/Cry transcription. Posttranscriptional modifications are heavily involved in clock oscillations and impart precision and robustness to the TTL. In particular, members of the casein kinase family (e.g., $\mathrm{CKI} \varepsilon, \mathrm{CKI} \delta$ ) are known to phosphorylate PER proteins at conserved residues and promote degradation, thereby delaying PER nuclear entry $[48,83]$. The auxiliary loop comprises two genes of the orphan nuclear receptor family, Rev-erb $\alpha$ (Nrldl) and Ror $\alpha$ (Rora). REV-ERB $\alpha$ and ROR $\alpha$ repress or activate, respectively, the transcription of genes with ROR elements in their promoters, such as Bmall and Npas2. Rev-erb $\alpha$ and Ror $\alpha$ are considered to be dispensable for cellular rhythm generation, yet they were shown to regulate phasing and amplitude of clock gene expression rhythms [116, 127]. Further ancillary loops have been described. The CLOCK/NPAS2-BMAL1-regulated bHLH transcription factors DEC1 (BHLHE40) and DEC2 (BHLHE41) were shown to bind E-box elements and modulate CLOCK/NPAS2/BMAL1-driven circadian transcription $[62,124]$. Another TTL involves the two transcription factors D-site albumin promoter binding protein and E4BP4 (NFIL3) that compete for binding of D-boxes, a third circadian regulatory DNA motif, at the promoters of Per1-3, Rev-erb $\alpha$, Ror $\alpha$, and various clockcontrolled genes (CCGs) [90, 101]. Similarly, in Drosophila, CYCLE (CYC) and CLOCK (CLK), the orthologs of BMAL1 and CLOCK, form heterodimers and activate transcription of the circadian repressor genes Timeless (Tim) and Period (Per) via E-boxes (Fig. 1, right side). TIM is a substitute for mammalian CRYs as the major core TTL inhibitor, whereas in the fly, CRY functions primarily as a photoreceptor and helps to synchronize the clock to the lightdark cycle [142]. TIM/PER complexes are transported to the nucleus to repress CYC/CLK-mediated transcription [56]. DOUBLETIME, a homolog of casein kinase I, phosphorylates PER, assigning it for sequestration via the proteasomal pathway $[72,117]$. Similar to mammals, the fly clock contains at least one auxiliary loop consisting of VRILLE (VRI) and PAR-domain protein-1 (PDP1). The former inhibits, whereas the latter activates $C l k$ transcription [26].

\section{Interaction between circadian and homeostatic sleep} components

The current model predicts that sleep is regulated by two principle mechanisms $[13,27]$. The first, termed process $c$, determines the appropriate timing of sleep. Nocturnal animals experience sleep mostly during the day, while diurnal species such as humans rest predominantly during the night. The SCN, as master circadian pacemaker, sends projections to important sleep regulatory nuclei such as the ventrolateral pre-optic area, the dorsomedial nucleus of the hypothalamus, and the hypocretin/orexin neurons of the lateral hypothalamus. Process $\mathrm{c}$ is complemented by a homeostatically controlled sleep drive, process s, which builds up an increased need for sleep in response to extended wake periods, independent of the time of day. So far, the anatomical substrate of process $\mathrm{s}$ remains elusive. Of note, sleep in mammals and birds is quantified primarily by electro-encephalography (EEG). In contrast, characterization of sleep in insects, where rhythm and homeostasis appear to be as robust as in mammals, relies mostly on measurements of rest/activity periods and arousal thresholds [60, 131]. SCN-lesioned rats and mice show disrupted sleep timing and consolidation, though the overall time spent asleep each day and the delta response to sleep deprivation remain uncompromised [67, 100, 147]. Previous studies conveyed on humans under forced desynchrony protocols demonstrated that slow wave activity was largely independent of internal circadian phase, though distribution of REM sleep and spindle activity in non-rapid eye movement (NREM) sleep correlated with body temperature rhythms [34]. At the same time, homeostatic sleep components can modify circadian pacemaker function. Sleep states affect activity of SCN neurons with decreasing firing rates during NREM phase and after sleep deprivation [30, 31]. Moreover, prolonged awaking effects the expression of clock genes in the cerebral cortex, upregulating both of Perl and Per $2[161,162]$. This body of evidence suggests that the circadian clock regulates sleep-wake timing and opposes process $\mathrm{s}$ in order to gate consolidated bouts of sleep and waking. 


\section{Clock genes and sleep timing}

In humans, naturally occurring polymorphisms in clock genes correlate with early or late chronotype. A PER3 gene length polymorphism is linked to extreme diurnal preferences [4]. The longer allele, which carries five repetitions $\left(P E R 3^{5 / 5}\right)$ of a variable number tandem repeat, is associated with early morning type, whereas the shorter allele $\left(P E R 3^{4 / 4}\right)$ correlates with eveningness and delayed sleep phase syndrome (DSPS). Recently, it was demonstrated that a polymorphism in the PER3 promoter is also associated with DSPS [6]. Similar correlations of polymorphic alleles with diurnal preferences are observed for PERI and the 5'untranslated region of PER2 [18, 19].

Circadian control of sleep can be better demonstrated on disorders associated with extremely shifted sleep-wake time. In familiar advanced sleep phase syndrome (FASPS), genetic studies identified mutations in the PER2 (S662G) and CKI (T44A) genes in some families [148, 164]. Remarkably, both mutations affect an evolutionary conserved process, the phosphorylation of the PER2 protein by CKI. Xu et al. [164] have shown that a single amino acid substitution (T44A) in the human CKI $\delta$ protein decreases its enzymatic activity in vitro. The corresponding mutation, when reproduced in mice, causes a shortened circadian period length, which is consistent with symptoms of FASPS patients. Surprisingly, a miss-sense mutation in the same conserved residue of the Drosophila CK1 ortholog Dbt leads to an increase in the free-running period [164], as would be expected from DSPS patients. These findings highlight the different organization of circadian/sleep regulatory mechanisms in insects and mammals, despite the fact that individual components share a great similarity between vertebrate and invertebrate systems (see also Fig. 1). In another study, transgenic mice expressing human PER2 with the S662G mutation on a Per2-deficient background display a shorter period resembling humans with FASPS. Conversely, an aspartate substitution at the same residue (S662D), mimicking a constitutively phosphorylated state, correlates with a longer period [165].

\section{Clock genes and sleep homeostasis}

A number of recent studies suggest that clock genes, besides regulating circadian sleep-wake timing, also contribute to sleep homeostatic control (reviewed in [47] and summarized in Table 1). Naylor et al. [105] demonstrated that mutations in Clock have effects on a variety of sleepwake parameters in the mouse. Clock mutant animals show a reduction in total sleep time (around $2 \mathrm{~h}$ ) under light/dark (LD) conditions, mostly due to reduced NREM sleep. In constant darkness (DD), homozygous mutants spend more time of their circadian cycle awake, mostly sacrificing NREM sleep, even when the results are normalized to their longer endogenous circadian period of $28.8 \mathrm{~h}$. The response to sleep deprivation is also altered in these mice with decreased REM sleep rebounds, though the effects on

Table 1 Clock gene mutant mice with sleep abnormalities

\begin{tabular}{|c|c|c|c|c|c|c|c|}
\hline \multirow[t]{2}{*}{ Mouse mutant } & \multirow{2}{*}{$\begin{array}{l}\text { Circadian } \\
\text { phenotype }\end{array}$} & \multicolumn{6}{|l|}{ Sleep phenotype } \\
\hline & & $\begin{array}{l}\text { Sleep amount, } \\
\text { light/dark phase }\end{array}$ & $\begin{array}{l}\text { REM, light/ } \\
\text { dark phase }\end{array}$ & $\begin{array}{l}\text { NREM, light/ } \\
\text { dark phase }\end{array}$ & $\begin{array}{l}\text { Delta power in } \\
\text { NREM, light/ } \\
\text { dark phase }\end{array}$ & $\begin{array}{l}\text { Response to sleep } \\
\text { deprivation }\end{array}$ & References \\
\hline Bmal1 $^{-1-}$ & Arrhythmic & Normal/elevated & Normal/elevated & Normal/elevated & Elevated/reduced & $\begin{array}{l}\text { Attenuated NREM/ } \\
\text { REM }\end{array}$ & {$[81]$} \\
\hline Npas2 $2^{-1-}$ & Short period & Normal/reduced & Normal/reduced & Normal/reduced & Normal/normal & $\begin{array}{l}\text { Attenuated NREM, } \\
\text { reduced delta } \\
\text { power }\end{array}$ & {$[39,46]$} \\
\hline Clock $^{\Delta 19}$ & Long period & Reduced/reduced & Normal/normal & Reduced/reduced & - & Attenuated REM & {$[105]$} \\
\hline $\operatorname{Per} 1 / 2^{m / m}$ & Arrhythmic & Reduced/normal & Normal/normal & Reduced/normal & Normal/normal & $\begin{array}{l}\text { Increased delta } \\
\text { power }\end{array}$ & [135] \\
\hline $\operatorname{Cry} 1 / 2^{-\leftarrow}$ & Arrhythmic & Normal/elevated & Reduced/elevated & Normal/elevated & Elevated/elevated & $\begin{array}{l}\text { Attenuated NREM/ } \\
\text { REM, reduced } \\
\text { delta power }\end{array}$ & {$[161]$} \\
\hline$D b p^{-1-}$ & Short period & Normal/normal & Reduced/normal & Normal/normal & Normal/reduced & Attenuated REM & {$[45]$} \\
\hline $\operatorname{Dec} 2^{P 385 R}$ & Normal & Reduced/normal & Reduced/normal & Reduced/normal & Normal/normal & $\begin{array}{l}\text { Attenuated NREM/ } \\
\text { REM, reduced } \\
\text { delta power }\end{array}$ & [59] \\
\hline$P K 2^{-/-}$ & $\begin{array}{l}\text { Attenuated } \\
\text { amplitude }\end{array}$ & Reduced/normal & Normal/elevated & Reduced/normal & Normal/normal & $\begin{array}{l}\text { Attenuated NREM/ } \\
\text { REM, reduced } \\
\text { delta power }\end{array}$ & {$[65]$} \\
\hline $\operatorname{Vipr} 2^{-\leftarrow}$ & Arrhythmic & Reduced/elevated & Reduced/elevated & Reduced/elevated & Normal/normal & - & [133] \\
\hline
\end{tabular}


NREM and total sleep are unchanged [105]. Gene association studies performed on two independent populations of humans report links between sequence variants of CLOCK and sleep duration [1]. In the clock machinery, Npas2 acts as a functional paralog of Clock, yet their expression in the brain rarely overlaps [2, 50, 71]. Consistent with this, Npas2-deficient mice show about $25 \%$ reduction in NREM and REM sleep as well as reduced sleep consolidation [39]. Subjected to $8 \mathrm{~h}$ of prolonged waking, Npas 2 mutants display a smaller compensatory increase in NREM sleep and in delta activity [46].

Bmall-deficient mice are to date the only reported mouse strain in which the deletion of a single gene totally disrupts circadian molecular and behavioral rhythms [17]. Consistent with their arrhythmic behavior, homozygous $\mathrm{Bmall}^{-/}$mice show attenuated sleep-wake rhythms and increased sleep fragmentation. In contrast to Clock mutants, Bmal1 $^{-/}$animals exhibit longer REM and NREM periods under LD and DD conditions. Furthermore, during the light phase delta power is constantly high, indicating that these animals are persistently under elevated sleep pressure. Paradoxically, when actively sleep deprived, Bmall mutants show an attenuated homeostatic response. This might be due to a lesser amount of sleep lost during sleep restriction when compared to wild-type animals [81].

Mice lacking both Cryl and Cry 2 genes are frequently used as a genetic model of circadian arrhythmicity [152, 157]. In Cryl $/ 2^{-/}$mice, sleep parameters do not differ between light and dark phases, indicating a non-circadian distribution of sleep. Both NREM sleep and EEG delta power are increased during the light phase, and Cryl/2 mutants also show attenuated responses to sleep restriction [161]. Of note, single Cryl or Cry2 knockouts do not display any significant differences in sleep parameters consistent with their - at least partially - redundant role in the circadian clock [162]. Similarly, Per gene mutations have only modest effects on sleep homeostasis. Studies done on both Perl and Per2 single mutant mice reveal altered 24-h distribution of sleep but normal responses to sleep deprivation $[76,135,162]$. However, behaviorally arrhythmic Per $1 / 2$ double mutant animals show decreased REM and NREM sleep periods during the light phase and moderately increased delta power after prolonged waking [135]. Remarkably, in rats, expression of Perl in the dorsomedial SCN was correlated with timing of REM sleep occurrence, pointing to a function of the central pacemaker itself in sleep architecture regulation [84]. In humans, the PER3 gene also plays a role in sleep homeostasis. Individuals bearing the gene length variant $P E R 3^{5 / 5}$ show longer durations of NREM sleep bouts, higher delta power, and an exaggerated response to sleep deprivation [155].
In Drosophila, mutants of mammalian clock gene orthologs also exhibit profound changes in sleep homeostasis. In particular $\mathrm{clk}^{j r k}$, $\mathrm{per}^{01}$, and $\mathrm{tim}^{01}$ flies show increased sleep rebounds after 7, 9, and $12 \mathrm{~h}$ of sleep deprivation and recover $100 \%$ (compared to $30-40 \%$ in wild-type Canton-S flies) of sleep lost within 12 h [132]. In turn, more tremendous sleep rebound and even lethality in response to $12 \mathrm{~h}$ of sleep deprivation have been observed in $c y c^{01}$ mutants [132]. Interestingly, this phenotype differs between genders with stronger effects seen in females [61].

Dbp knockout mice were the first animal model investigated for the role of clock genes in sleep homeostasis [45]. In constant darkness, Dbp-deficient mice exhibit a slightly shorter free-running period and decreased overall activity [90]. On EEG recordings $D b p$ mutants show reduced REM sleep during the light phase as well as less delta power activity in the dark. After $6 \mathrm{~h}$ of sleep restriction, significantly attenuated REM responses are observed [45]. In a recent study, He and co-workers [59] found $D E C 2$ to regulate sleep length in humans. They identified a miss-sense mutation in the human $D E C 2$ gene that is associated with a sleep phenotype (6 vs. $8 \mathrm{~h}$ sleep duration in control subjects). When this point substitution is reproduced in mice, it decreases total sleep time via both REM and NREM without affecting circadian period [59]. A targeted deletion of $D e c 2$, however, does only produce a mild sleep phenotype in mice [59]. Interestingly, in the fly homolog of DEC2, CLOCKWORK ORANGE (CWO) [87], the affected amino acid residue (P385) is not conserved, but flies expressing a mutant mouse $D e c 2$ show a similar sleep phenotype [59].

Of note, some of the genes known to mediate transcriptional output from the circadian clock machinery have also been implicated in sleep regulation. For instance, targeted deletion of Prokineticin2 (Pk2), encoding a peptide secreted from the SCN and critical for the maintenance of robust circadian behavioral rhythms [24, 86], produces profound alterations in sleep homeostasis. $P k 2$ mutants show a $20 \%$ reduction in total sleep time, mostly due to a decrease in NREM sleep in the light phase, whereas REM sleep is increased. Delta power and REM sleep rebound after sleep deprivation are also attenuated in these mice [65]. A recent study on the VPAC2 subtype of the VIP receptor (VIPR2) implicated in the coupling of cellular oscillators within the SCN pacemaker demonstrates the significance of synchronization of electrical activity in SCN neurons on sleep regulation. Consistent with locomotor activity data, an attenuated diurnal rhythm of sleep and wakefulness is seen in Vipr2-deficient mice, although total sleep time and other homeostatic parameters are not affected [58, 133]. Interestingly, in flies, a disruption of the VIP analog neuropeptide pigment dispersing factor (PDF) increases sleep and causes reduced responsiveness to external stimuli [25]. Together, 
these studies clearly show that circadian and homeostatic regulatory circuits show a high degree of interaction. It remains unclear, however, how this cross-talk is mediated at the molecular level and which neuronal circuits are involved.

\section{Clock genes and sleep-associated functions}

Clock genes do not only influence sleep architecture and quality. They might also be involved in sleep and sleepcorrelated functions within an organism [51]. In many cases, clock gene mutations and sleep disorders share the same symptoms and phenotypes. Sleep loss, for example, has been correlated to numerous metabolic symptoms, which are also observed in circadian clock-deficient animal models. Clock genes affect synaptic plasticity in learning and memory formation and modulate immune functions during the course of the day. In the same way, sleep - or the lack thereof - has a strong impact on these processes.

\section{Energy metabolism}

The efficient regulation of energy homeostasis is an essential factor for an organism's survival. It comprises a range of different processes, including energy uptake (i.e. eating), storage (mostly as lipids, glycogen or tissue protein), and expenditure (energy usage for biosynthetic processes, heat production or locomotion). Energy is taken up in the form of macronutrients - carbohydrates, fat, or protein. In most species, nutrient ingestion follows a strict circadian rhythm, and several clock genes have been shown to be involved in the regulation of metabolic homeostasis. Clock mutant mice show blunted diurnal activity rhythms resulting in elevated food intake during the usual resting phase (day) and less ingestion during the active phase (night). These mice become hyperphagic and obese [151]. Another study showed that daytime high fat diet (HFD) in mice leads to a significant higher weight gain than nighttime HFD [3]. This is a possible explanation for the clock mutants' obese phenotype. Similarly, Per 2 mutant mice show arrhythmic feeding behavior and eat significantly more under (HFD) conditions. These effects are based on a decreased level of alpha melanocyte-stimulating hormone ( $\alpha$-MSH), a well-known appetite suppressor, at the beginning of the light phase. Constant administration of $\alpha$-MSH to Per 2 mutants leads to reduced food uptake, revealing $\alpha$-MSH as a direct target of the clock gene Per2, independent of rhythmicity [167]. Interestingly, some of these effects are also seen after sleep restriction in rodents and in humans. In the latter, a restriction of sleep time to $4 \mathrm{~h}$ for only a few consecutive nights is enough to significantly increase appetite [139, 140]. Rats that are kept awake for 2 weeks using the classic discover-water technique show hyperphagy - although they lose weight under these conditions [119]. Of note, a number of other animal studies failed to confirm an increase in food uptake in response to sleep restriction, indicating that small variations in experimental procedures may have a significant impact on these processes $[9,170]$. A straight-forward mechanistic explanation for a sleep-loss-mediated increase in energy uptake remains elusive. It was suggested that a temporal deregulation of peripheral orexigenic and anorexigenic hormones could underlie this effect. The most promising candidates are the gastrointestinal peptide ghrelin $[29,73]$ and the adipokine leptin secreted by white adipocytes [89]. In humans as well as in animals, sleep restriction or total sleep deprivation cause significant decreases in circulating leptin and increased ghrelin, thus promoting appetite and hunger [11, 44, 140, 145]. Human leptin plasma levels are partially dependent on meal time [128] and also on the circadian time and sleep state. Under un-stressed and constant feeding conditions, leptin shows a marked nocturnal rise in humans [128]. When sleeping time is shifted by $8 \mathrm{~h}$, leptin levels are differentially regulated by both the circadian system and sleep, resulting in a short period rhythm with peaks in the night and around mid-sleep phase [136]. In contrast, the diurnal expression of ghrelin seems not to be directly clock-regulated. Under ad libitum feeding conditions ghrelin shows a bimodal rhythm with peaks in the afternoon and towards the end of the dark phase in rats, correlating with gastric emptying and filling [103]. However, in humans, sleep triggers ghrelin release during night. Comparable to rats, humans also show a bimodal ghrelin rhythm with one peak in the afternoon and one peak during night. The peak during night is absent when test persons were sleep deprived [40]. Ghrelin signaling seems to have a strong influence on the circadian system. In cultured mouse brain slices containing the SCN, ghrelin administration increases firing rate of individual SCN neurons. Ghrelin receptor activation phase shifts $\mathrm{SCN}$ bioluminescence rhythms in culture and resets locomotor activity rhythms in intact mice [168]. Another agent connecting the circadian system, sleep, and food uptake is the neuropeptide orexin (or hypocretin/HCRT). Its two isoforms, orexin A and B, are exclusively expressed in neurons of the lateral hypothalamus. Both have potent wake-promoting effects and at the same time stimulate food intake [126]. Orexin release is circadian clock-controlled and Hcrt transcription rhythms are abolished in Clock mutant mice [151]. The SCN directly innervates orexigenic neurons [104]. Under starvation conditions, the sleep duration of rats is shortened [28], while sleep deprivation increases energy uptake [119]. The orexin system constitutes a potential candidate linking both processes. 
Similar to food uptake, energy metabolism is influenced by the circadian system and sleep. Both sleep and clock disruptions have strong effects on glucose and lipid metabolism. Several clock gene mutant mouse strains show phenotypes resembling aspects of the type II diabetes pathology. The overexpression of mutant Cryl protein results in polydipsia, polyuria, and hyperglycemia [109]. Clock mutant mice show hyperglycemia and hypoinsulinemia [151]. Moreover, Clock mutant and Bmall $^{-/}$mice exhibit impaired glucose liberation from the liver. Under HFD conditions, these mice show deficient insulin regulation and beta cell function in the pancreas [125]. A liver-specific deletion of Bmall promotes hypoglycemia and deregulated expression of genes involved in glucose metabolism, such as phosphoenolpyruvate carboxykinase $1(P c k 1)$, glucokinase $(G c k)$, and glucose-6-phosphate translocase 1 (G6pt1/Slc37a4) [79]. The fact that polymorphisms in the Clock gene are associated with metabolic syndrome in man and that several Bmall haplotypes in rats are connected to type II diabetes underlines the connection between circadian genes and metabolism [130, 163]. CCG mutations can also cause diabetic symptoms. Nocturnin is a clockcontrolled deadenylase involved in post-transcriptional regulation of gene expression. Loss of Nocturnin (Ccrnl4) has strong effects on insulin sensitivity and glucose tolerance [53]. Other examples are the orphan nuclear receptor peroxisome proliferator-activated receptor $\alpha$ (Ppara) and tumor necrosis factor alpha (TNF- $\alpha$ ) $[54,108]$. Strikingly similar effects on metabolism have been attributed to sleep (or the lack thereof). The global trend towards shorter sleep times during the last decades was suggested as one of the factors underlying the alarming increase in the prevalence of the metabolic syndrome and type II diabetes $[52,106]$. In line with this, poor sleep quality is a risk factor for type II diabetes [146]. Experimentally, restriction of sleep to $4 \mathrm{~h}$ per night for less than a week increases blood glucose levels while at the same time decreasing insulin sensitivity [139], suggesting that a chronic reduction of sleep time raises the risk of developing diabetes.

Other processes associated with clock gene function are lipid metabolism in adipocytes and energy expenditure in the muscles. Clock mutant mice suffer from hyperlipidemia [151], and Bmall is necessary for adipocyte differentiation from mouse embryonic fibroblast cultures. Restoration of BMAL1-expression in Bmal1-deficient 3T3-L1 precursor cells rescues adipogenesis. A treatment with PPAR $\gamma$ ligands reconstitutes the differentiation potential of these cells. In addition, many other lipid metabolism-related genes, like $a P 2, S R E B P-1 \alpha$, and perilipin, are effected by Bmall restoration, indicating that all these genes are direct targets of Bmall [134]. Interestingly, Bmall ${ }^{-/}$mice exhibit no alterations in body weight gain under a variety of diet conditions. However, a possible obesity phenotype in these animals might be confounded by their premature aging [74]. The Bmall regulator $\mathrm{ROR} \alpha$ promotes fatty acid oxidation via its targets, caveolin-3 and CPT-1, lipogenesis, and lipid storage in skeletal muscles [82], while Nocturnindeficient mice show resistance to diet-induced obesity [53]. Although the molecular mechanisms are less well understood, several studies suggest that shortened sleep also has a strong influence on lipid metabolism. A chronic lack of sleep, either shortened sleep time or poor sleep quality, is a strong risk factor for obesity and the development of the metabolic syndrome [49, 69]. In a large longitudinal study on nurses, Patel et al. [112] observed a cross-sectional Ushaped association between sleep duration and body mass index (BMI) development over several years. It was suggested that sleep effects on lipid metabolism are mediated by endocrine factors, such as cortisol, prolactin, or insulin, as well as by sympathetic hyperactivity, which had previously been linked to obesity and insulin resistance. In this manner, sleep restriction represents a minor form of chronic stress, thus activating the sympathicus and elevating epinephrine and norepinephrine secretion from the adrenal medulla. In line with this, sympathetic activation and catecholamine administration inhibit leptin expression and secretion [137], increase free fatty acid levels [66], and decrease insulin sensitivity [94]. Restricted sleep and sleep deprivation elevate glucocorticoid levels [85], further promoting visceral fat deposition and insulin resistance [122].

Several endocrine factors have been suggested as potential modulators of clock and sleep regulated aspects of energy metabolism, including adrenal glucocorticoids, pituitary hormones, as well as the "night hormone" melatonin. In most mammals and birds, melatonin is produced in the pineal gland during the night. The pineal receives direct and indirect signals from the SCN and is, therefore, rhythmically locked to the circadian master clock. Bi-directional links have been described between melatonin production and the regulation of glucose metabolism. In diabetic GotoKakizaki (GK) rats, melatonin levels are significantly reduced, while melatonin receptor expression in the pancreas is increased [113]. In line with this, melatonin signaling has a strong influence on insulin secretion from the pancreatic beta cells [102].

\section{Neuronal plasticity}

Clock gene-sleep interactions have also been reported in the context of neuronal plasticity and learning processes [51]. These include both short and long-term memory 
(LTM) formation and recall. The latter is thought to depend primarily on hippocampal long-term potentiation (LTP), a form of synaptic plasticity [96]. LTP efficiency is time-ofday dependent in the hippocampus and in the SCN [10, 22, 107]. Several studies show that circadian core clock genes are involved in long-term memory formation. Mutations of Npas 2 cause impaired LTM in a fear conditioning paradigm [50]. Several clock output factors also have a role in memory formation. Inhibition of melatonin or deletion of the gene encoding the SCN-secreted peptide vasoactive intestinal polypeptide (VIP) disrupts memory formation $[23,159]$. These phenotypes are often connected to cAMP signaling $[158,169]$. MAPK phosphorylation and cAMP and CREB phosphorylation are clock gene controlled in the hippocampus. The nadir of these events corresponds to the time when the strongest learning deficits are observed and pharmacological inhibition of MAPK phosphorylation during a learning task impairs memory formation [41]. Like memory formation, memory recall is under direct influence of clock genes. Cryl/2 $2^{-/}$mice show normal spatial memory and perform well in simple avoidance tasks. They are, however, unable to efficiently learn in a more complex time-place context [153]. Similar findings were reported from Per2 mutant animals [160]. In a foodrewarded hippocampus-dependent spatial memory task (eight-arm radial maze) [129], $\mathrm{Per}^{-/}$mice fail more frequently than wild-type littermates [70]. Various publications show strong influences of sleep on memory. For instance, individuals carrying homozygous $\mathrm{Per} 3^{5 / 5}$ alleles exhibit an interesting connection among their clock genotype, sleep, and regional brain response patterns to an executive task. In contrast to PER $3^{4 / 4}$ participants, sleep deprivation correlates to changes in brain activity only in $P E R 3^{5 / 5}$ participants [154].

Although the influence of sleep on memory processes has primarily been studied in a neurophysiological rather than in a molecular context, there are several common features of sleep and clock gene impact on memory processes. The hypothesis that sleep has a positive influence on memory formation is not new [68]. Numerous studies provide evidence that both declarative and procedural memory processes benefit from proper sleep $[95,138]$. Even very short naps of a few minutes have been shown to improve declarative and procedural memory formation and recall $[77,78,99$, 150]. Re-entrainment of sleep patterns can restore cognitive functions either in transgenic mice carrying the Huntington's disease mutation [111] or in elderly patients showing symptoms of dementia [121]. However, until now, it is not fully clarified whether sleep has, like clock gene function, an influence on the formation of LTP. LTP can occur during REM sleep [15], and REM deprivation impairs LTP in the rat hippocampus [123].
On the other hand, REM deprivation does not necessary lead to disturbed memory formation [118]. Total sleep deprivation can lead to problems in learning [32], but this effect seems to be highly dependent on the subjects' general cognitive capacity [33]. In animals, current sleep deprivation protocols always include a certain stress component, which in itself can interfere with memory formation [63].

\section{Immune functions}

Similar to the brain, the immune system acts as a bidirectional interface between the organism and its environment. From a more general perspective, it also functions in a very similar way in terms of detection of, reaction to, and memorization of information. Sleep has a strong influence on the immune system and vice versa [93]. Inflammation state affects sleep time and quality in animals and humans $[16,30,38]$, while sleep restriction leads to higher mortality rates upon infection or sepsis [42, 43, 149]. Several immune parameters show circadian rhythmicity in the blood of humans and other mammals [80]. Clock gene expression is rhythmic in peripheral leukocytes [5]. Moreover, the secretion of important neuroendocrine immune modulators is under circadian as well as under sleep control. The activities of the hypothalamus pituitary adrenal (HPA) axis and the sympathetic nervous system, both stress activated, are influenced by the circadian system and sleep. In arrhythmic Per2/Cryl double mutant mice, HPA axis regulation is strongly affected. The responsiveness of the adrenal to adrenocorticotropin stimulation and, thus, the production of glucocorticoids are regulated by adrenocortical circadian clocks [110]. Humans show elevated cortisol and norepinephrine levels in response to sleep deprivation while epinephrine levels become arrhythmic [80]. These effects will likely give rise to changes observed in leukocyte production. For leukocytes, strong diurnal rhythms have been reported [14, 144], some of which seem to directly respond to cortisol secretion during night time and to epinephrine during the day $[36,37]$. Moreover, sleep loss affects levels of lymphocytes, monocytes, natural killer (NK) cells, and T-cell proliferation in humans $[12,14,35,97]$. Not only the appearance of immune cells is circadian as well as sleep-controlled but also the levels of several cytokines [93]. While interleukin (IL)-6 production seems to primarily depend on clock function, rhythmic TNF- $\alpha$, IL-10, and IL12 release from monocytes as well as IL-12 production by dendritic cells depend critically on sleep-wake conditions [80].

Circadian rhythm disruption has been shown to severely weaken the immune system. Mice exposed to four consecutive weekly 6 -h phase advances of the light/dark 
schedule (a repetitive jet lag paradigm) show increased mortality in response to lipopolysaccharide (LPS) injection [20]. Likewise, the clock gene Per2 has been identified to play a direct role in the activation of macrophages by controlling the expression of interferon- $\gamma(\mathrm{IFN}-\gamma)$, a macrophage activating factor, in the mouse spleen [7]. The same is true for the natural killer (NK) cell receptors LY49C and NKG2D [91]. NK cell-specific knockdown of Per 2 leads to decreased protein levels of the immune factors granzyme B and perforin in rats, but not of IFN- $\gamma$ [8]. Interestingly, LPS administration in Per2-deficient mice provokes attenuated immune responses and yields considerable higher survival rates than in wild-type animals [88]. Bmall $^{-/-}$mice show significantly reduced levels of $B$ cells in peripheral blood, spleen and bone marrow [143]. PER $3^{5 / 5}$ individuals show elevated IL-6 concentrations compared to those with the $P E R^{4 / 4}$ genotype [55]. As for metabolism, melatonin secretion might be one of the messengers linking sleep, circadian system, and immune function. Chemical inhibition of melatonin secretion leads to decreased antibody responses in mice. This effect is reversed by melatonin administration [92]. Melatonin further promotes the production of macrophage and granulocyte progenitor cells and affects the production of cytokines, such as IL-1, IL-2, IL-6, IL-12, TGF- $\beta$, M-CSF, and TNF- $\alpha$ [141]. Vice versa, cytokines might have influence on clock gene expression. TNF- $\alpha$ and IL-1 $\beta$ suppress the expression of Perl-3 and Rev-erb $\alpha$ in fibroblasts and liver of mice [21] in a p38 MAP kinase and calcium-dependent way [114]. This impairment of clock genes might lead to increased fatigue seen after infections.

Both sleep deprivation and clock mutations deregulate the production of pro-inflammatory cytokines, and low-grade systemic inflammation is a known pathological component of obesity and metabolic syndrome [64, 98]. Moreover, the production of IL-1 is increased in humans with self-reported poor sleep quality. Remarkably, this correlation of sleep debt and IL-1 levels does not apply for obese humans $(\mathrm{BMI} \geq 30)$ [115]. Elderly people with metabolic syndrome and systemic inflammation show a higher risk of cognitive impairment compared with those without metabolic syndrome or with metabolic syndrome but without inflammation [166]. This leads to the suggestion of a direct connection between sleep, circadian clock genes, metabolism, cognition, and the immune system.

\section{Conclusions}

Although it seems clear that sleep and clock genes have strong influences on various physiological processes, it is often technically difficult to disentangle clock gene, circadian rhythm, and sleep-specific influences because of their mutual dependency and because only little so far is known about the underlying mechanisms and circuits (summarized in Fig. 2). While, in this paper, we have independently evaluated the impact of both processes, it is well conceivable that some of the physiological functions of one might be mediated via regulation of the other, i.e., sleep regulates clocks regulate physiology or clocks regulate sleep regulate physiology - and vice versa. While tremendous progress has been made in deciphering the molecular machinery of circadian clocks, little is still known of how different tissue oscillators communicate with each other to synchronize behavior and physiology. Even less understood are the processes underlying sleep. In fact, the tight interaction between clocks and sleep processes and the surprisingly strong effect that clock gene mutations have on both processes $\mathrm{c}$ and $\mathrm{s}$ might provide new inroads into mapping the sleep circuitry of the brain and into identifying molecular substrates of sleep within neuronal cells. With the advent of conditional genetics in mice and functional brain imaging techniques in humans, new tools have been developed to more specifically address these issues in the living organism. On the other hand, improved experimental paradigms need to be developed to resolve some of the conflicting findings from animal and human studies. The pathological long-term effects of the progressing sleeplessness and circadian desynchrony of modern societies will be in the focus of future research. A better understanding of the underlying mechanisms might well become a key for advanced therapeutic strategies against some of the most pressing health issues such as diabetes and neuropsychiatric disorders.

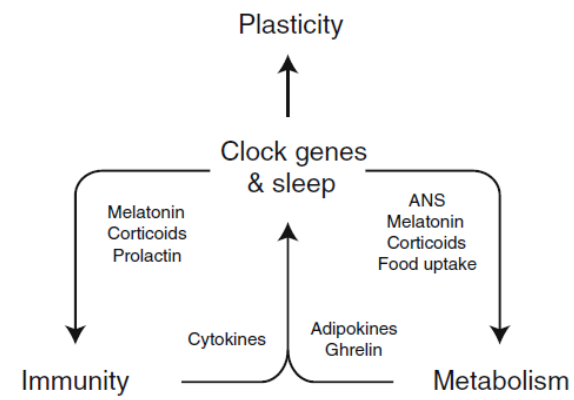

Fig. 2 Interaction of sleep and clocks in the regulation of cognitive and physiological processes. A reciprocal interactivity exists between sleep and circadian clock function. Both neuronal and blood-borne factors have been proposed to mediate clocksleep and sleep-physiology communication. On the other hand, peripheral humoral factors have been shown to provide feedback about the physiological state to sleep and clock regulatory circuits. The local regulation of physiology by tissue clocks may serve to integrate sleep state and timing signals at the cellular level. For details, see text 
Acknowledgments We would like to thank Johanna Barclay for critical reading of the manuscript. H.O. is an Emmy Noether fellow of the German Research Foundation (DFG). A.S. is supported by the Max Planck Society (MPG).

\section{References}

1. Allebrandt KV et al (2010) CLOCK gene variants associate with sleep duration in two independent populations. Biol Psychiatry 67 (11):1040-1047

2. Antoch MP et al (1997) Functional identification of the mouse circadian Clock gene by transgenic BAC rescue. Cell 89(4):655-667

3. Arble DM et al (2009) Circadian timing of food intake contributes to weight gain. Obesity (Silver Spring) 17 (11):2100-2102

4. Archer SN et al (2003) A length polymorphism in the circadian clock gene Per3 is linked to delayed sleep phase syndrome and extreme diumal preference. Sleep 26(4):413-415

5. Archer SN et al (2008) Inter-individual differences in habitual sleep timing and entrained phase of endogenous circadian rhythms of BMAL1, PER2 and PER3 mRNA in human leukocytes. Sleep 31(5):608-617

6. Archer SN et al (2010) Polymorphism in the PER3 promoter associates with diurnal preference and delayed sleep phase disorder. Sleep 33(5):695-701

7. Arjona A, Sarkar DK (2006) The circadian gene mPer2 regulates the daily rhythm of IFN-gamma. J Interferon Cytokine Res 26 (9):645-649

8. Arjona A, Sarkar DK (2006) Evidence supporting a circadian control of natural killer cell function. Brain Behav Immun 20 (5):469-476

9. Balestrieri S, D'Onofrio G, Giuditta A (1980) Deprivation of paradoxical sleep. Effect on weight and nucleic acid content of liver and brain. Neurochem Res 5(12):1251-1264

10. Barnes CA et al (1977) Circadian rhythm of synaptic excitability in rat and monkey central nervous system. Science 197(4298):91-92

11. Bodosi B et al (2004) Rhythms of ghrelin, leptin, and sleep in rats: effects of the normal diurnal cycle, restricted feeding, and sleep deprivation. Am J Physiol Regul Integr Comp Physiol 287 (5):R1071-R1079

12. Bollinger $T$ et al (2009) Sleep-dependent activity of $T$ cells and regulatory T cells. Clin Exp Immunol 155(2):231-238

13. Borbely AA (1982) A two process model of sleep regulation. Hum Neurobiol 1(3):195-204

14. Born J et al (1997) Effects of sleep and circadian rhythm on human circulating immune cells. J Immunol 158(9):4454-4464

15. Bramham CR, Srebro B (1989) Synaptic plasticity in the hippocampus is modulated by behavioral state. Brain Res 493 (1):74-86

16. Bryant PA, Trinder J, Curtis N (2004) Sick and tired: does sleep have a vital role in the immune system? Nat Rev Immunol 4 (6):457-467

17. Bunger MK et al (2000) Mop3 is an essential component of the master circadian pacemaker in mammals. Cell 103 (7): 1009-1017

18. Carpen JD et al (2005) A single-nucleotide polymorphism in the 5 -untranslated region of the hPER2 gene is associated with diurnal preference. J Sleep Res 14(3):293-297

19. Carpen JD et al (2006) A silent polymorphism in the PER 1 gene associates with extreme diurnal preference in humans. J Hum Genet 51(12):1122-1125
20. Castanon-Cervantes O et al (2010) Dysregulation of inflammatory responses by chronic circadian disruption. J Immunol 185 (10):5796-5805

21. Cavadini G et al (2007) TNF-alpha suppresses the expression of clock genes by interfering with E-box-mediated transcription. Proc Natl Acad Sci USA 104(31):12843-12848

22. Chaudhury D, Wang LM, Colwell CS (2005) Circadian regulation of hippocampal long-term potentiation. J Biol Rhythms 20(3):225-236

23. Chaudhury D et al (2008) Select cognitive deficits in vasoactive intestinal peptide deficient mice. BMC Neurosci 9:63

24. Cheng MY et al (2002) Prokineticin 2 transmits the behavioura circadian rhythm of the suprachiasmatic nucleus. Nature 417 (6887):405-410

25. Chung BY et al (2009) The GABA(A) receptor RDL acts in peptidergic PDF neurons to promote sleep in Drosophila. Curr Biol 19(5):386-390

26. Cyran SA et al (2003) vrille, Pdp1, and dClock form a second feedback loop in the Drosophila circadian clock. Cell 112 (3):329-341

27. Daan S, Beersma DG, Borbely AA (1984) Timing of human sleep: recovery process gated by a circadian pacemaker. Am J Physiol 246(2 Pt 2):R161-R183

28. Danguir J, Nicolaidis S (1979) Dependence of sleep on nutrients' availability. Physiol Behav 22(4):735-740

29. Date $Y$ et al (2000) Ghrelin, a novel growth hormone-releasing acylated peptide, is synthesized in a distinct endocrine cell type in the gastrointestinal tracts of rats and humans. Endocrinology 141(11):4255-4261

30. Deboer $\mathrm{T}$ et al (2003) Sleep states alter activity of suprachiasmatic nucleus neurons. Nat Neurosci 6(10):1086-1090

31. Deboer T, Detari L, Meijer JH (2007) Long term effects of sleep deprivation on the mammalian circadian pacemaker. Sleep 30 (3):257-262

32. Diekelmann S et al (2008) Sleep loss produces false memories. PLoS One 3(10):e3512

33. Diekelmann S, Born J, Wagner U (2010) Sleep enhances false memories depending on general memory performance. Behav Brain Res 208(2):425-429

34. Dijk DJ, Czeisler CA (1995) Contribution of the circadian pacemaker and the sleep homeostat to sleep propensity, sleep structure, electroencephalographic slow waves, and sleep spindle activity in humans. J Neurosci Off J Soc Neurosci $15(5 \mathrm{Pt}$ 1):3526-3538

35. Dimitrov $\mathrm{S}$ et al (2007) Number and function of circulating human antigen presenting cells regulated by sleep. Sleep 30 (4):401-411

36. Dimitrov S et al (2009) Cortisol and epinephrine control opposing circadian rhythms in $\mathrm{T}$ cell subsets. Blood 113 (21):5134-5143

37. Dimitrov S, Lange T, Born J (2010) Selective mobilization of cytotoxic leukocytes by epinephrine. J Immunol 184(1):503-511

38. Drake CL et al (2000) Effects of an experimentally induced rhinovirus cold on sleep, performance, and daytime alertness. Physiol Behav 71(1-2):75-81

39. Dudley CA et al (2003) Altered patterns of sleep and behavioral adaptability in NPAS2-deficient mice. Science 301 (5631):379-383

40. Dzaja A et al (2004) Sleep enhances nocturnal plasma ghrelin levels in healthy subjects. Am J Physiol Endocrinol Metab 286 (6):E963-E967

41. Eckel-Mahan KL et al (2008) Circadian oscillation of hippocampal MAPK activity and cAmp: implications for memory persistence. Nat Neurosci 11(9):1074-1082

42. Everson CA (1993) Sustained sleep deprivation impairs host defense. Am J Physiol 265(5 Pt 2):R1148-R1154 
43. Everson CA, Toth LA (2000) Systemic bacterial invasion induced by sleep deprivation. Am J Physiol Regul Integr Comp Physiol 278(4):R905-R916

44. Everson CA, Crowley WR (2004) Reductions in circulating anabolic hormones induced by sustained sleep deprivation in rats. Am J Physiol Endocrinol Metab 286(6):E1060-E1070

45. Franken $P$ et al (2000) The transcription factor DBP affects circadian sleep consolidation and rhythmic EEG activity. J Neurosci 20(2):617-625

46. Franken P et al (2006) NPAS2 as a transcriptional regulator of non-rapid eye movement sleep: genotype and sex interactions. Proc Natl Acad Sci USA 103(18):7118-7123

47. Franken P, Dijk DJ (2009) Circadian clock genes and sleep homeostasis. Eur J Neurosci 29(9):1820-1829

48. Gallego M, Virshup DM (2007) Post-translational modifications regulate the ticking of the circadian clock. Nat Rev Mol Cell Biol 8(2):139-148

49. Gangwisch JE et al (2005) Inadequate sleep as a risk factor for obesity: analyses of the NHANES I. Sleep 28(10):1289-1296

50 . Garcia JA et al (2000) Impaired cued and contextual memory in NPAS2-deficient mice. Science 288(5474):2226-2230

51. Gerstner JR (2010) The aging clock: to 'BMAL'icious toward learning and memory. Aging 2(5):251-254

52. Gottlieb DJ et al (2005) Association of sleep time with diabetes mellitus and impaired glucose tolerance. Arch Intern Med 165 (8):863-867

53. Green CB et al (2007) Loss of Nocturnin, a circadian deadenylase, confers resistance to hepatic steatosis and diet-induced obesity. Proc Natl Acad Sci USA 104(23):9888-9893

54. Grimble RF (2002) Inflammatory status and insulin resistance. Curr Opin Clin Nutr Metab Care 5(5):551-559

55. Guess J et al (2009) Circadian disruption, Per3, and human cytokine secretion. Integr Cancer Ther 8(4):329-336

56. Hardin PE (2005) The circadian timekeeping system of Drosophila. Curr Biol 15(17):R714-R722

57. Hardin PE, Hall JC, Rosbash M (1990) Feedback of the Drosophila period gene product on circadian cycling of its messenger RNA levels. Nature 343(6258):536-540

58. Harmar AJ et al (2002) The VPAC(2) receptor is essential for circadian function in the mouse suprachiasmatic nuclei. Cell 109 (4):497-508

59. He Y et al (2009) The transcriptional repressor DEC2 regulates sleep length in mammals. Science 325(5942):866-870

60. Hendricks JC et al (2000) Rest in Drosophila is a sleep-like state. Neuron 25(1):129-138

61. Hendricks JC et al (2003) Gender dimorphism in the role of cycle (BMAL1) in rest, rest regulation, and longevity in Drosophila melanogaster. J Biol Rhythms 18(1):12-25

62. Honma S et al (2002) Dec1 and Dec2 are regulators of the mammalian molecular clock. Nature 419(6909):841-844

63. Horne JA, McGrath MJ (1984) The consolidation hypothesis for REM sleep function: stress and other confounding factors - a review. Biol Psychol 18(3):165-184

64. Hotamisligil GS, Erbay E (2008) Nutrient sensing and inflammation in metabolic diseases. Nat Rev Immunol 8 (12):923-934

65. Hu WP et al (2007) Altered circadian and homeostatic sleep regulation in prokineticin 2-deficient mice. Sleep 30(3):247-256

66. Hucking K et al (2003) Burst-like control of lipolysis by the sympathetic nervous system in vivo. J Clin Invest 111 (2):257-264

67. Ibuka N, Nihonmatsu I, Sekiguchi S (1980) Sleep-wakefulness rhythms in mice after suprachiasmatic nucleus lesions. Waking Sleeping 4(2):167-173

68. Jenkins JG, Dallenbach KM (1924) Obliviscence during sleep and waking. Am J Psychol 35:605-612
69. Jennings JR et al (2007) Self-reported sleep quality is associated with the metabolic syndrome. Sleep 30(2):219-223

70. Jilg A et al (2010) Temporal dynamics of mouse hippocampal clock gene expression support memory processing. Hippocampus 20(3):377-388

71. King DP et al (1997) Positional cloning of the mouse circadian clock gene. Cell 89(4):641-653

72. Ko HW, Jiang J, Edery I (2002) Role for Slimb in the degradation of Drosophila Period protein phosphorylated by Doubletime. Nature 420(6916):673-678

73. Kojima M et al (1999) Ghrelin is a growth-hormone-releasing acylated peptide from stomach. Nature 402(6762):656-660

74. Kondratov RV et al (2006) Early aging and age-related pathologies in mice deficient in BMAL1, the core componentof the circadian clock. Genes Dev 20(14):1868-1873

75. Konopka RJ, Benzer S (1971) Clock mutants of Drosophila melanogaster. Proc Natl Acad Sci USA 68(9):2112-2116

76. Kopp C et al (2002) Homeostatic sleep regulation is preserved in mPer1 and mPer2 mutant mice. Eur J Neurosci 16(6):1099-1106

77. Korman M et al (2007) Daytime sleep condenses the time course of motor memory consolidation. Nat Neurosci 10(9):1206-1213

78. Lahl O et al (2008) An ultra short episode of sleep is sufficient to promote declarative memory performance. J Sleep Res 17(1):3-10

79. Lamia KA, Storch KF, Weitz CJ (2008) Physiological significance of a peripheral tissue circadian clock. Proc Natl Acad Sci USA 105(39):15172-15177

80. Lange T, Dimitrov S, Born J (2010) Effects of sleep and circadian rhythm on the human immune system. Ann N Y Acad Sci 1193:48-59

81. Laposky A et al (2005) Deletion of the mammalian circadian clock gene BMAL1/Mop3 alters baseline sleep architecture and the response to sleep deprivation. Sleep 28(4):395-409

82. Lau P et al (2004) RORalpha regulates the expression of genes involved in lipid homeostasis in skeletal muscle cells: caveolin-3 and CPT-1 are direct targets of ROR. J Biol Chem 279(35):36828-36840

83. Lee $\mathrm{C}$ et al (2001) Posttranslational mechanisms regulate the mammalian circadian clock. Cell 107(7):855-867

84. Lee ML, Swanson BE, de la Iglesia HO (2009) Circadian timing of REM sleep is coupled to an oscillator within the dorsomedial suprachiasmatic nucleus. Curr Biol 19(10):848-852

85. Leproult $\mathrm{R}$ et al (1997) Sleep loss results in an elevation of cortisol levels the next evening. Sleep 20(10):865-870

86. Li JD et al (2006) Attenuated circadian rhythms in mice lacking the prokineticin 2 gene. J Neurosci 26(45):11615-11623

87. Lim C et al (2007) Clockwork orange encodes a transcriptional repressor important for circadian-clock amplitude in Drosophila. Curr Biol 17(12):1082-1089

88. Liu J et al (2006) The circadian clock Period 2 gene regulates gamma interferon production of NK cells in host response to lipopolysaccharide-induced endotoxic shock. Infect Immun 74 (8): $4750-4756$

89. Lonnqvist $\mathrm{F}$ et al (1995) Overexpression of the obese (ob) gene in adipose tissue of human obese subjects. Nat Med 1(9):950-953

90. Lopez-Molina L et al (1997) The DBP gene is expressed according to a circadian rhythm in the suprachiasmatic nucleus and influences circadian behavior. EMBO J 16(22):6762-6771

91. Luo $Y$ et al (2009) Expression profiling reveals a positive regulation by $\mathrm{mPer} 2$ on circadian rhythm of cytotoxicity receptors: Ly49C and Nkg2d. Chronobiol Int 26(8):1514-1544

92. Maestroni GJ, Conti A, Pierpaoli W (1986) Role of the pineal gland in immunity. Circadian synthesis and release of melatonin modulates the antibody response and antagonizes the immunosuppressive effect of corticosterone. J Neuroimmunol 13(1):19-30 
93. Majde JA, Krueger JM (2005) Links between the innate immune system and sleep. J Allergy Clin Immunol 116(6):1188-1198

94. Marangou AG et al (1988) Hormonal effects of norepinephrine on acute glucose disposal in humans: a minimal model analysis. Metabolism 37(9):885-891

95. Marshall L, Born J (2007) The contribution of sleep to hippocampus-dependent memory consolidation. Trends Cogn Sci 11(10):442-450

96. Martin SJ, Grimwood PD, Morris RG (2000) Synaptic plasticity and memory: an evaluation of the hypothesis. Annu Rev Neurosci 23:649-711

97. Matsumoto Y et al (2001) Total sleep deprivation induces an acute and transient increase in NK cell activity in healthy young volunteers. Sleep 24(7):804-809

98. Maury E, Ramsey KM, Bass J (2010) Circadian rhythms and metabolic syndrome: from experimental genetics to human disease. Circ Res 106(3):447-462

99. Mednick S, Nakayama K, Stickgold R (2003) Sleep-dependent learning: a nap is as good as a night. Nat Neurosci 6(7):697-698

100. Mistlberger RE et al (1983) Recovery sleep following sleep deprivation in intact and suprachiasmatic nuclei-lesioned rats. Sleep 6(3):217-233

101. Mitsui S et al (2001) Antagonistic role of E4BP4 and PAR proteins in the circadian oscillatory mechanism. Genes Dev 15 (8):995-1006

102. Mulder $\mathrm{H}$ et al (2009) Melatonin receptors in pancreatic islets: good morning to a novel type 2 diabetes gene. Diabetologia 52 (7): $1240-1249$

103. Murakami $\mathrm{N}$ et al (2002) Role for central ghrelin in food intake and secretion profile of stomach ghrelin in rats. J Endocrinol 174 (2):283-288

104. Nambu T et al (1999) Distribution of orexin neurons in the adult rat brain. Brain Res 827(1-2):243-260

105. Naylor E et al (2000) The circadian clock mutation alters sleep homeostasis in the mouse. J Neurosci 20(21):8138-8143

106. Nilsson PM et al (2004) Incidence of diabetes in middleaged men is related to sleep disturbances. Diabetes Care 27 (10):2464-2469

107. Nishikawa Y, Shibata S, Watanabe S (1995) Circadian changes in long-term potentiation of rat suprachiasmatic field potentials elicited by optic nerve stimulation in vitro. Brain Res 695 (2): $158-162$

108. Oishi K, Shirai H, Ishida N (2005) CLOCK is involved in the circadian transactivation of peroxisome-proliferator-activated receptor alpha (PPARalpha) in mice. Biochem J 386 (Pt 3):575-581

109. Okano S et al (2009) Unusual circadian locomotor activity and pathophysiology in mutant CRY1 transgenic mice. Neurosci Lett 451(3):246-251

110. Oster $\mathrm{H}$ et al (2006) The circadian rhythm of glucocorticoids is regulated by a gating mechanism residing in the adrenal cortical clock. Cell Metab 4(2):163-173

111. Pallier PN et al (2007) Pharmacological imposition of sleep slows cognitive decline and reverses dysregulation of circadian gene expression in a transgenic mouse model of Huntington's disease. J Neurosci Off J Soc Neurosci 27 (29):7869-7878

112. Patel SR et al (2004) A prospective study of sleep duration and mortality risk in women. Sleep 27(3):440-444

113. Peschke E et al (2006) Diabetic Goto Kakizaki rats as well as type 2 diabetic patients show a decreased diurnal serum melatonin level and an increased pancreatic melatonin-receptor status. J Pineal Res 40(2):135-143

114. Petrzilka $\mathrm{S}$ et al (2009) Clock gene modulation by TNF-alpha depends on calcium and p38 MAP kinase signaling. J Biol Rhythms 24(4):283-294
115. Prather AA et al (2009) Normative variation in self-reported sleep quality and sleep debt is associated with stimulated proinflammatory cytokine production. Biol Psychol 82(1):12-17

116. Preitner $\mathrm{N}$ et al (2002) The orphan nuclear receptor REVERBalpha controls circadian transcription within the positive limb of the mammalian circadian oscillator. Cell 110(2):251-260

117. Price JL et al (1998) Double-time is a novel Drosophila clock gene that regulates PERIOD protein accumulation. Cell 94 (1):83-95

118. Rasch B et al (2009) Pharmacological REM sleep suppression paradoxically improves rather than impairs skill memory. Nat Neurosci 12(4):396-397

119. Rechtschaffen A, Bergmann BM (1995) Sleep deprivation in the rat by the disk-over-water method. Behav Brain Res 69(1-2):55-63

120. Reppert SM, Weaver DR (2002) Coordination of circadian timing in mammals. Nature 418(6901):935-941

121. Riemersma-van der Lek RF et al (2008) Effect of bright light and melatonin on cognitive and noncognitive function in elderly residents of group care facilities: a randomized controlled trial. JAMA 299(22):2642-2655

122. Roberge $\mathrm{C}$ et al (2007) Adrenocortical dysregulation as a major player in insulin resistance and onset of obesity. Am J Physiol Endocrinol Metab 293(6):E1465-E1478

123. Romcy-Pereira R, Pavlides C (2004) Distinct modulatory effects of sleep on the maintenance of hippocampal and medial prefrontal cortex LTP. Eur J Neurosci 20(12):3453-3462

124. Rossner MJ et al (2008) Disturbed clockwork resetting in Sharp-1 and Sharp-2 single and double mutant mice. PLoS One 3(7):e2762

125. Rudic RD et al (2004) BMAL1 and CLOCK, two essential components of the circadian clock, are involved in glucose homeostasis. PLoS Biol 2(11):e377

126. Sakurai T (2005) Roles of orexin/hypocretin in regulation of sleep/wakefulness and energy homeostasis. Sleep Med Rev 9 (4):231-241

127. Sato TK et al (2004) A functional genomics strategy reveals Rora as a component of the mammalian circadian clock. Neuron 43(4):527-537

128. Schoeller DA et al (1997) Entrainment of the diurnal rhythm of plasma leptin to meal timing. J Clin Invest 100(7):1882-1887

129. Schwegler H, Crusio WE, Brust I (1990) Hippocampal mossy fibers and radial-maze learning in the mouse: a correlation with spatial working memory but not with non-spatial reference memory. Neuroscience 34(2):293-298

130. Scott EM, Carter AM, Grant PJ (2008) Association between polymorphisms in the Clock gene, obesity and the metabolic syndrome in man. Int J Obes (Lond) 32(4):658-662

131. Shaw PJ et al (2000) Correlates of sleep and waking in Drosophila melanogaster. Science 287(5459):1834-1837

132. Shaw PJ et al (2002) Stress response genes protect against lethal effects of sleep deprivation in Drosophila. Nature 417 (6886):287-291

133. Sheward WJ et al (2010) Circadian control of mouse heart rate and blood pressure by the suprachiasmatic nuclei: behavioral effects are more significant than direct outputs. PLoS One 5(3):e9783

134. Shimba $\mathrm{S}$ et al (2005) Brain and muscle Amt-like protein-1 (BMAL1), a component of the molecular clock, regulates adipogenesis. Proc Natl Acad Sci USA 102(34):12071-12076

135. Shiromani PJ et al (2004) Sleep rhythmicity and homeostasis in mice with targeted disruption of mPeriod genes. Am J Physiol Regul Integr Comp Physiol 287(1):R47-R57

136. Simon $C$ et al (1998) Circadian and ultradian variations of leptin in normal man under continuous enteral nutrition: relationship to sleep and body temperature. J Clin Endocrinol Metab 83 (6): 1893-1899 
137. Slieker LJ et al (1996) Regulation of expression of ob mRNA and protein by glucocorticoids and cAMP. J Biol Chem 271 (10):5301-5304

138. Smith C (2001) Sleep states and memory processes in humans: procedural versus declarative memory systems. Sleep Med Rev 5 (6):491-506

139. Spiegel K, Leproult R, Van Cauter E (1999) Impact of sleep debt on metabolic and endocrine function. Lancet 354 (9188):1435-1439

140. Spiegel K et al (2004) Brief communication: sleep curtailment in healthy young men is associated with decreased leptin levels, elevated ghrelin levels, and increased hunger and appetite. Ann Intem Med 141(11):846-850

141. Srinivasan $\mathrm{V}$ et al (2005) Melatonin, immune function and aging. Immun Ageing 2:17

142. Stanewsky R et al (1998) The cryb mutation identifies cryptochrome as a circadian photoreceptor in Drosophila. Cell 95(5):681-692

143. Sun Y et al (2006) MOP3, a component of the molecular clock, regulates the development of B cells. Immunology 119 (4): $451-460$

144. Suzuki S et al (1997) Circadian rhythm of leucocytes and lymphocytes subsets and its possible correlation with the function of the autonomic nervous system. Clin Exp Immunol 110(3):500-508

145. Taheri S et al (2004) Short sleep duration is associated with reduced leptin, elevated ghrelin, and increased body mass index. PLoS Med 1(3):e62

146. Tasali E, Mokhlesi B, Van Cauter E (2008) Obstructive sleep apnea and type 2 diabetes: interacting epidemics. Chest 133 (2):496-506

147. Tobler I, Borbely AA, Groos G (1983) The effect of sleep deprivation on sleep in rats with suprachiasmatic lesions. Neurosci Lett 42(1):49-54

148. Toh KL et al (2001) An hPer2 phosphorylation site mutation in familial advanced sleep phase syndrome. Science 291 (5506): 1040-1043

149. Toth LA, Tolley EA, Krueger JM (1993) Sleep as a prognostic indicator during infectious disease in rabbits. Proc Soc Exp Biol Med 203(2):179-192

150. Tucker MA et al (2006) A daytime nap containing solely non-REM sleep enhances declarative but not procedura memory. Neurobiol Learn Mem 86(2):241-247

151. Turek FW et al (2005) Obesity and metabolic syndrome in circadian Clock mutant mice. Science 308(5724):1043-1045

152. van der Horst GT et al (1999) Mammalian Cry1 and Cry2 are essential for maintenance of circadian rhythms. Nature 398 (6728):627-630

153. Van der Zee EA et al (2008) Circadian time-place learning in mice depends on Cry genes. Curr Biol 18(11):844-848
154. Vandewalle $\mathrm{G}$ et al (2009) Functional magnetic resonance imaging-assessed brain responses during an executive task depend on interaction of sleep homeostasis, circadian phase, and PER3 genotype. J Neurosci Off J Soc Neurosci 29 (25):7948-7956

155. Viola AU et al (2007) PER3 polymorphism predicts sleep structure and waking performance. Curr Biol 17(7):613-618

156. Vitaterna $\mathrm{MH}$ et al (1994) Mutagenesis and mapping of a mouse gene, Clock, essential for circadian behavior. Science 264 (5159):719-725

157. Vitaterna $\mathrm{MH}$ et al (1999) Differential regulation of mammalian period genes and circadian rhythmicity by cryptochromes 1 and 2. Proc Natl Acad Sci USA 96(21):12114-12119

158. von Gall C et al (1998) CREB in the mouse SCN: a molecular interface coding the phase-adjusting stimuli light, glutamate, PACAP, and melatonin for clockwork access. J Neurosci 18 (24):10389-10397

159. Wang LM et al (2005) Melatonin inhibits hippocampal long-term potentiation. Eur J Neurosci 22(9):2231-2237

160. Wang, LM et al. (2009) Expression of the circadian clock gene Period2 in the hippocampus: possible implications for synaptic plasticity and learned behaviour. ASN Neuro. 1(3):e00012

161. Wisor JP et al (2002) A role for cryptochromes in sleep regulation. BMC Neurosci 3:20

162. Wisor JP et al (2008) Sleep deprivation effects on circadian clock gene expression in the cerebral cortex parallel electroencephalographic differences among mouse strains. J Neurosci 28 (28):7193-7201

163. Woon PY et al (2007) Aryl hydrocarbon receptor nuclear translocator-like (BMAL1) is associated with susceptibility to hypertension and type 2 diabetes. Proc Natl Acad Sci USA 104 (36): 14412-14417

164. Xu Y et al (2005) Functional consequences of a CKIdelta mutation causing familial advanced sleep phase syndrome. Nature 434(7033):640-644

165. Xu Y et al (2007) Modeling of a human circadian mutation yields insights into clock regulation by PER2. Cell 128(1):59-70

166. Yaffe $\mathrm{K}$ et al (2004) The metabolic syndrome, inflammation, and risk of cognitive decline. JAMA 292(18):2237-2242

167. Yang S et al (2009) The role of mPer2 clock gene in glucocorticoid and feeding rhythms. Endocrinology 150 (5):2153-2160

168. Yannielli PC et al (2007) Ghrelin effects on the circadian system of mice. J Neurosci 27(11):2890-2895

169. Yin JC et al (1995) CREB as a memory modulator: induced expression of a dCREB2 activator isoform enhances long-term memory in Drosophila. Cell 81(1):107-115

170. Youngblood BD et al (1999) The effects of paradoxical sleep deprivation and valine on spatial learning and brain 5-HT metabolism. Physiol Behav 67(5):643-649 


\section{Acknowledgements}

I would like thank my mentor, Prof. Dr. Henrik Oster, for giving me the opportunity to work under his supervision. First of all, I really appreciate his continuous support and advice he gave me for the last four years. He created a remarkable working atmosphere which inspired me and other members of our group for new ideas and fruitful scientific discussions. Prof. Oster encouraged me to expand my knowledge about biological clocks and to proceed with my scientific career in circadian biology.

I also would like to thank Prof. Dr. Gregor Eichele for his help and generous support. He provided me with all facilities and materials and funded my travels to conferences in India, UK and USA.

I also want to express my gratitude to the members of my thesis committee, Prof. Dr. Ahmed Mansouri and Prof. Dr. Ernst Wimmer for their helpful suggestions and encouragement.

Thanks to all former and current members of the Circadian Rhythms Group for the nice and friendly environment. I really enjoyed the time with people who shared with me our productive journal clubs and symposia: Alexei Leliavski, Judit Meyer-Kovac, Johanna Barclay, Jana Husse, Silke Kiessling, Dominic Landgraf, Brid Bode and Anthony Tsang. Thanks to Nadine Naujokat, Christin Helbig, and our animal caretaker Alexandra Driehorst for their help with genotyping. I also would like to thank all members of the Department Genes and Behavior. Special thanks to Nikolai Petkau for his friendship and help with cloning. I also would like to acknowledge our administrative staff, Helena Miletic and Christine van den Bogaart, for their courtesy and organizational support.

Last but not least thanks to my wife Vera, who was able to finish her apprenticeship while still finding time to raise our two wonderful sons Lukas and Leon, who were born during my thesis work. They gave their enormous love and support to me during this period. I also would like to thank my mother Liudmila, father Ryhor and brother Ihar for their help and encouragement.

Anton. 


\section{CURRICULUM VITAE}

\section{Personal data}

Name:

Date of birth:

Place of birth:

\section{Education}

Nov 2008-Oct 2012

Jan 2008 - Oct 2008

Sep 2002 -Jun 2007

Sep 2005 - Aug 2007

Nov 2004 - Jun 2007

Diploma practical training at the Institute of Bioorganic Chemistry of the

Sep 1992 - Jun 2002 Secondary School № 108, Minsk, Belarus National Academy of Sciences of Belarus, Minsk, Belarus.

Completed diploma thesis "Interaction of NADPH-cytochrome P450 reductase with myoglobin/hemoglobin"

Doctoral Student Researcher in the Department of Genes and Behaviour, Max Planck Institute for Biophysical Chemistry, Göttingen, Germany $\mathrm{PhD}$ thesis title: The role of adipose tissue circadian clocks in metabolic homeostasis

Research assistant at the Institute for Human Genetics, University of Göttingen, Germany

International Sakharov Environmental University (ISEU), Minsk, Belarus.

Diploma in Environmental Medicine and Radiobiology

Belarusian State University (BSU), Minsk, Belarus

Diploma in Translation 


\section{Completed publications}

Benedict C, Shostak A, Lange T, Brooks SJ, Schiöth HB, Schultes B, Born J, Oster H, Hallschmid M. Diurnal rhythm of circulating nicotinamide phosphoribosyltransferase (Nampt/visfatin/PBEF): impact of sleep loss and relation to glucose metabolism. J Clin Endocrinol Metab. 2012

Landgraf D*, Shostak A, Oster H. Clock genes and sleep. Pflugers Arch. 2012

Husse J, Zhou X, Shostak A, Oster H, Eichele G. Synaptotagmin10-Cre, a driver to disrupt clock genes in the SCN. J Biol Rhythms. 2011

* - equal contribution

\section{Manuscripts in preparation}

Shostak A, Oster H. Regulation of lipid mobilization by a circadian clock residing in white adipose tissues. Manuscript to be submitted

Barclay JL, Shostak A, Leliavski A , Landgraf D, Naujokat N, van der Horst G, Oster H. Altered energy homeostasis in Cry deficient mice. Manuscript to be submitted

\section{Meetings, Conferences and Lectures}

The 2012 Meeting of the Society for Research on Biological Rhythms, Destin, Florida, USA, 19-23 May, 2012 (oral presentation)

Leopoldina Symposium "The Circadian System: from Chronobiology to Chronomedicine" Frankfurt am Main, Germany, 23-24 March, 2012 (poster presentation)

MPI-bpc Campus Seminar "White adipose circadian clocks regulate lipolysis and energy homeostasis" 7 December 2011

13th International EMBL PhD Symposium "The Rhythm of Life ", Heidelberg, Germany, 17-19 November 2011 (poster presentation)

XII Congress of the European Biological Rhythms Society, Oxford, UK, 20-26 August 2011 (oral presentation)

The 5th EUclock European PhD School for Chronobiology, Bangalore, India, 2-9 October 2010 (oral presentation) 